\title{
Targeting the Nt17 of the huntingtin protein via natural and chemical modifications: impact on aggregation and membrane interactions
}

\author{
Faezeh Sedighi \\ WVU, fasedighi@mix.wvu.edu
}

Follow this and additional works at: https://researchrepository.wvu.edu/etd

Part of the Biochemistry, Biophysics, and Structural Biology Commons, and the Molecular and Cellular Neuroscience Commons

\section{Recommended Citation}

Sedighi, Faezeh, "Targeting the Nt17 of the huntingtin protein via natural and chemical modifications: impact on aggregation and membrane interactions" (2020). Graduate Theses, Dissertations, and Problem Reports. 7934.

https://researchrepository.wvu.edu/etd/7934

This Dissertation is protected by copyright and/or related rights. It has been brought to you by the The Research Repository @ WVU with permission from the rights-holder(s). You are free to use this Dissertation in any way that is permitted by the copyright and related rights legislation that applies to your use. For other uses you must obtain permission from the rights-holder(s) directly, unless additional rights are indicated by a Creative Commons license in the record and/ or on the work itself. This Dissertation has been accepted for inclusion in WVU Graduate Theses, Dissertations, and Problem Reports collection by an authorized administrator of The Research Repository @ WVU.

For more information, please contact researchrepository@mail.wvu.edu. 
Targeting the Nt17 domain of the huntingtin protein via natural and chemical modifications: impact on aggregation and membrane interactions

\author{
Faezeh Sedighi \\ Dissertation submitted to the \\ Eberly College of Arts and Science \\ at West Virginia University \\ in partial fulfillment of the requirements for the degree of \\ Doctor of Philosophy in \\ Chemistry \\ Justin Legleiter, Ph.D., Committee Chairperson \\ Kenneth Showalter, Ph.D. \\ Michelle Richard-Babb, Ph.D. \\ Stephan J. Valentine, Ph.D. \\ Werner Geldenhuys, Ph.D. \\ C. Eugene Bennett Department of Chemistry \\ Morgantown, West Virginia \\ 2020
}

Keywords: Huntington disease, Huntingtin protein, protein aggregation, fibrils, oligomers, SUMOylation, crosslinking, lipid membrane

Copyright 2020

Faezeh Sedighi 


\section{Abstract \\ Targeting the Nt17 domain of the huntingtin protein via natural and chemical modifications: impact on aggregation and membrane interactions}

\section{Faezeh Sedighi}

Huntington Disease (HD) is a fatal neurodegenerative disorder caused by an expanded polyglutamine domain (polyQ) in the first exon of the huntingtin protein (htt-exon1). The major hallmark of HD is the accumulation of aggregates into proteinaceous inclusion bodies. PolyQ expansion in huntingtin promotes self-assembly into a variety of toxic aggregates such as oligomers, fibrils, and amorphous aggregates. The resulting heterogeneous mixture of distinct species makes it difficult to assign toxic function to specific aggregate structures. In addition, htt interacts with a variety of membranous surfaces. The first 17 amino acids (Nt17) of htt directly flanking the polyQ domain functions in binding lipids and in promoting aggregation based on its ability to form an amphipathic $\alpha$-helix. Nt17 undergoes several posttranslational modifications that modulate aggregation, subcellular localization, and toxicity of mutant htt. To gain in-depth mechanistic insights into huntingtin aggregation at lipid interfaces, both natural and chemical Nt17 modification strategies were employed. Specifically, the direct impact of SUMOylation was characterized. SUMOylation promoted the formation of large, SDS-soluble amorphous aggregates of htt and significantly inhibited the ability of htt to bind lipid membranes. In addition, the interaction of various htt aggregates species with lipid membrane was determined, and oligomers displayed the largest membrane activity. To further investigate these htt oligomers, a crosslinking strategy was employed that targeted lysine residues within Nt17. Crosslinking htt oligomers compromises their conformational flexibility, inhibiting their membrane activity. Cellular toxicity of crosslinked oligomers was also reduced, suggesting membrane activity may underlie mechanisms associated with htt oligomers. 


\section{Dedication}

I dedicated this dissertation to my parents, Reza and Shahin for their constant support and unconditional love. You were my biggest inspiration through my education. 


\section{Acknowledgments}

Undertaking this Ph.D. has been a truly life-changing challenge for me, and this dissertation would not have been completed without the support and guidance that I received from many people. My deepest gratitude goes to my $\mathrm{PhD}$ advisor, Dr. Justin Legleiter, who expertly guided me through my graduate education. I am forever grateful for the time, untiring support, and patience he has invested in me during the last five years. He has provided me with insightful discussions about the research, and his unwavering enthusiasm for science kept me constantly engaged with my research. He always had faith in me, even when I did not have faith in myself. I am genuinely grateful for the opportunity to develop assays and write protocols in the lab. Thanks to him, besides data analysis and critical thinking skills, I will benefit from the writing and presentation skills for the rest of my life. Dr. Legleiter is the best advisor I could have asked for my PhD. I am honestly lost for words to describe my gratitude.

My appreciation also extends to all my committee members for their constructive feedback and comments on my dissertation. I would especially like to thank Dr. Valentine, and his group members (Daud Sharif, Kushani Attanayake, and Ahmad Kiani) for helping me through my research. I am grateful for the time that Dr. Valentine patiently trained me on HPLC, as well as mass spectrometry and data analysis. I owe a debt of gratitude to Dr. Werner Geldenhuys for the invaluable help through the second project while times were tough during the pandemic. My last project would not be nearly as good without his help. I would also like to thank Dr. Kenneth Showalter for all the positive energy and interesting topics during his class. I would like to recognize Dr. Michelle Richard-Bab for kindly accepting to be my committee member and her time and feedback through the process. I would also like to thank Dr. David Smit, Jane Schupp, 
and Raymond Anderson in the health Science Center for providing me with training and lab facilities during my research.

I would also like to thank the other members of Dr. Legleiter's group for their support and collaboration through my research. I thank Dr. Maxmore Chaibva and Dr. Albert Pilkington for initially training me on lab techniques and helping me with assays troubleshooting. I was lucky to have the best lab mate of all-time, doctor-to-be Adewale Adegbuyiro. I wouldn't have been able to survive without his support and positive outlook. Thanks for the stimulating discussions, and the fun time we have had together in the last five years. I also thank Chathuranga Siriwardhana for all the support and offering to help through the research.

I am also thankful for my dearest friend, Kacee Caster. She was the one who helped me out during my first week in WVU when I was overwhelmed with the immigration and $\mathrm{PhD}$ program. She is a strong and big-hearted woman who provided me support when I was going through a rough time. I hope I can return the favor one day. I am thankful for my best friend Dr. Golnoosh Golpayegani, who stood by me for the last five years, listened to me and provided me with moral support like a sister. Above the ground, I am indebted to my family for unconditional love and support throughout my life. I especially thank my sister Flor Sedighi. She has been my best friend all my life. Her wisdom is invaluable, and I can't thank her enough for her support. I know I can always count on her when times are rough. 


\section{Table of Contents}

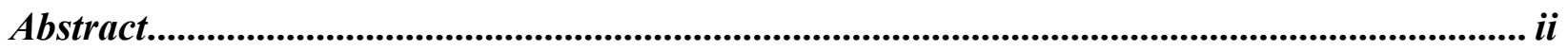

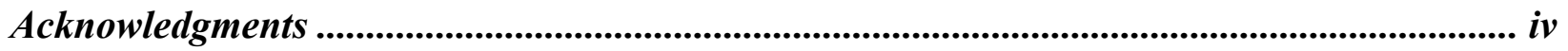

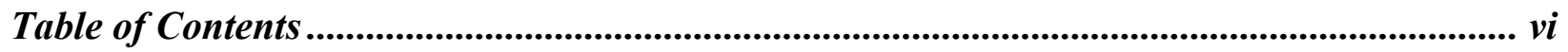

Table of Figures.....................................................................................................................

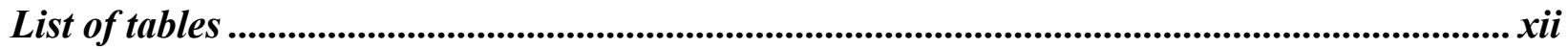

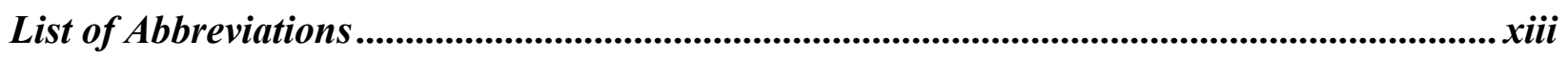

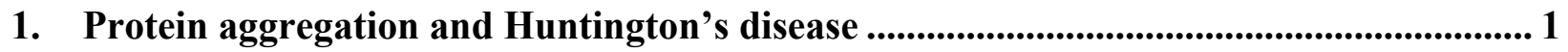

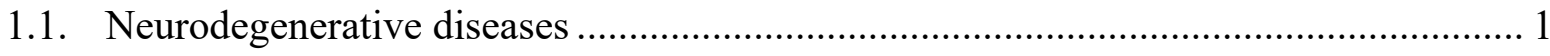

1.2. Amyloid formation in neurodegenerative disease............................................ 1

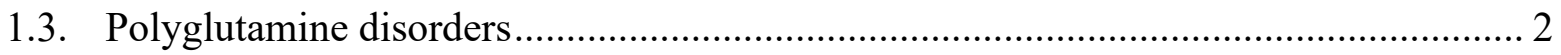

1.4. Huntingtin protein (htt) associated with Huntington's disease (HD) ........................ 4

1.5. Huntingtin forms a variety of aggregate species ............................................... 5

1.6. Ambiguity in the toxicity associated with variety of mutant htt species ................... 8

1.6.1. Protective role of Inclusion bodies ....................................................... 8

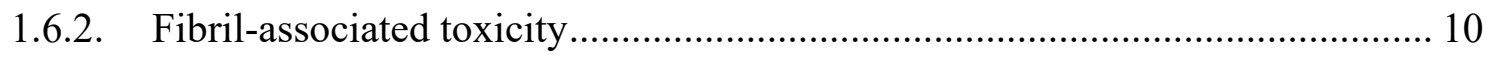

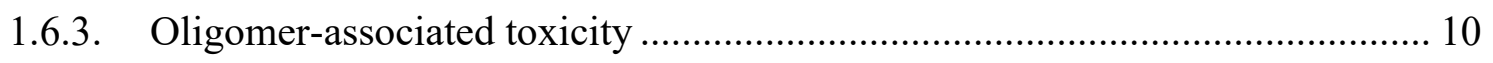

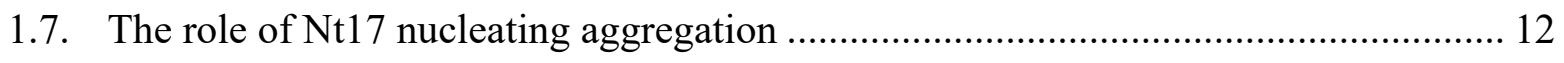

1.8. Htt membrane localization is implicated in toxic mechanism ................................ 14

1.9. Nt17 Function as a lipid binding domain ................................................... 16 
1.10. Importance of lysine residues in $\mathrm{Nt} 17$ 16

1.11. Post translational modification of Nt17 alter htt aggregation and lipid binding..... 18

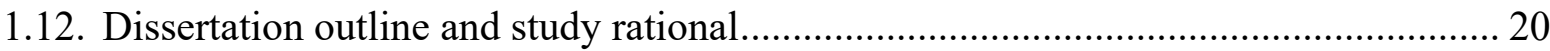

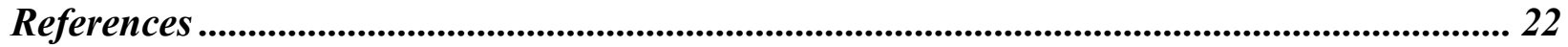

2. SUMOylation Prevents Huntingtin Fibrillization and Localization onto Lipid

Membranes .......................................................................................................................................... 31

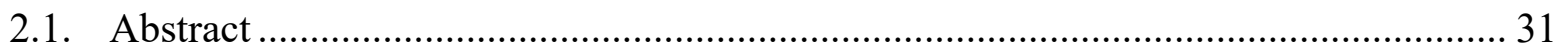

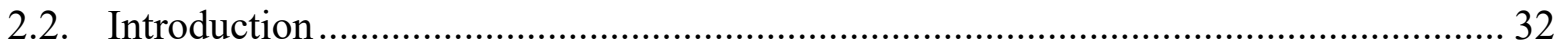

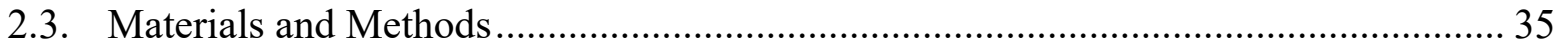

2.3.1. Expression and purification of GST-htt-exon1 fusion protein ......................... 35

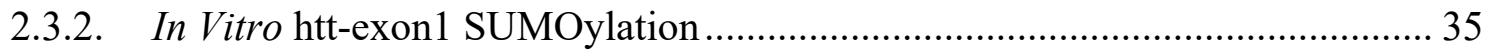

2.3.3. Thioflavin-T Fluorescence Assay (ThT) ………………………………….... 36

2.3.4. Circular Dichroism (CD) Spectroscopy ........................................................ 37

2.3.5. Filter Retardation and Dot Blot Assay ............................................................. 37

2.3.6. Atomic Force Microscopy (AFM) …………………................................ 38

2.3.7. TBLE/PDA Lipid Vesicle Protein Binding Assay.............................................. 40

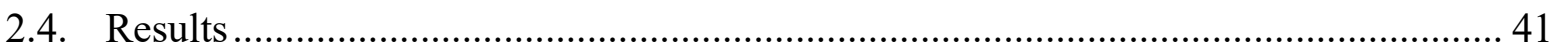

2.4.1. Htt-exon1(46Q) is readily SUMOylated in Vitro ............................................. 41

2.4.2. SUMOylation inhibits htt-exon1 fibrils formation in Vitro and alters aggregate

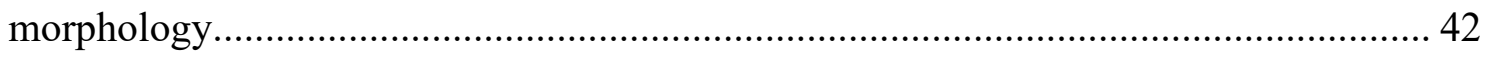

2.4.3. Secondary Structure of Aggregated htt Is Altered by SUMOylation ................ 50

2.4.4. SUMOylation Promotes the Formation of SDS Soluble Aggregates ............... 52 
2.4.5. SUMOylation Inhibits htt-Lipid Interaction

2.5. Discussion

References.

3. Oligomerization enhances huntingtin membrane activity but is suppressed by covalent crosslinking

3.1. Abstract 74

3.2. Introduction 75

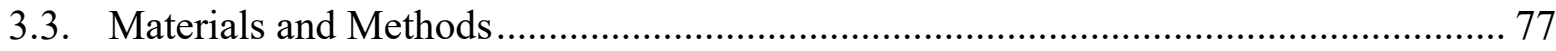

3.3.1. Expression and Purification of GST-htt-exon1 Fusion Protein ....................... 77

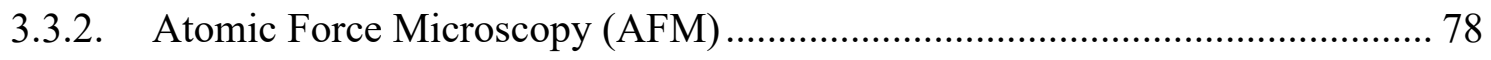

3.3.3. Polydiacetylene Lipid Binding Assay .................................................... 79

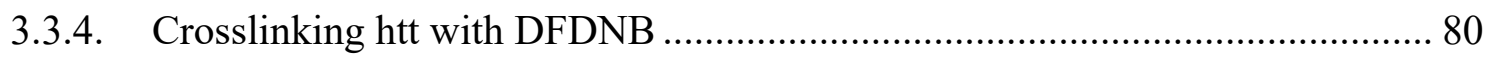

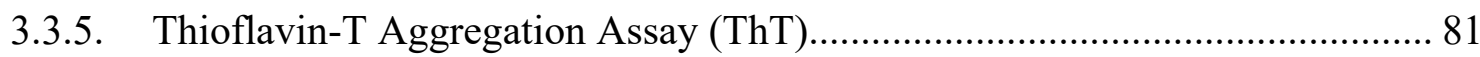

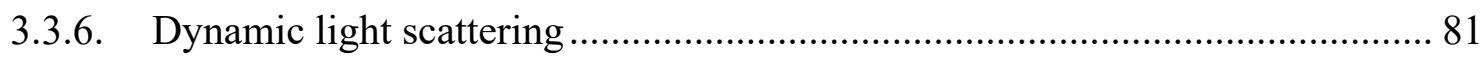

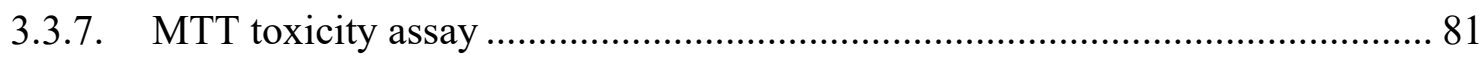

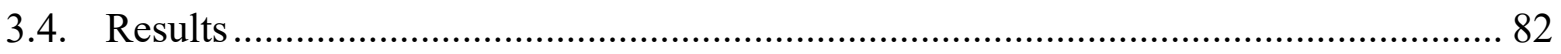

3.4.1. Htt oligomer formation correlates with lipid binding ............................... 82

3.4.2. Crosslinking oligomers alters htt aggregation in a dose-dependent manner .... 86

3.4.3. Crosslinking inhibits the ability of oligomers to bind to lipid vesicles ........... 90

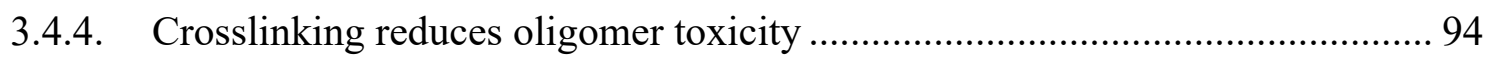

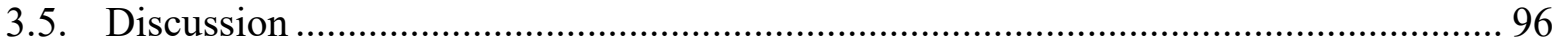

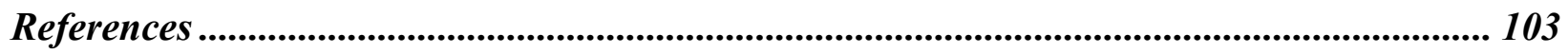




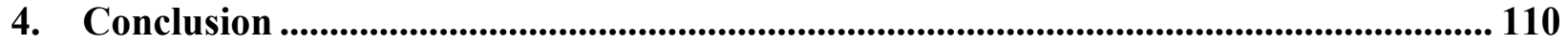

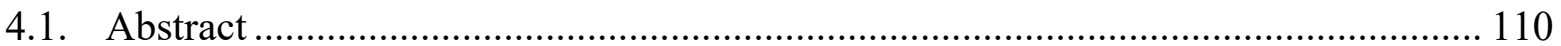

4.2. The role of heterogeneous htt aggregates in toxicity .......................................... 111

4.3. Mechanistic-based approaches to investigate htt aggregation pathway and lipid

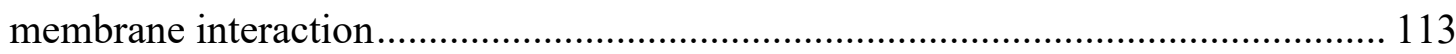

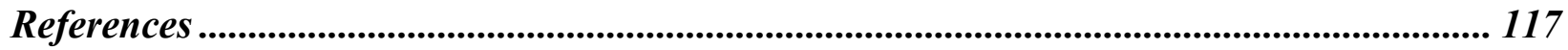




\section{Table of Figures}

Fig 1.1 The full-length htt protein contains several HEAT repeats.......................................... 5

Fig 1.2 Schematic representation of the amyloid aggregation via nucleation-dependent

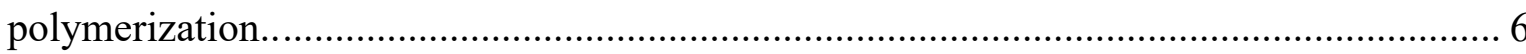

Fig 1.3 A schematic model for misfolding and aggregation of mutant htt protein...................... 8

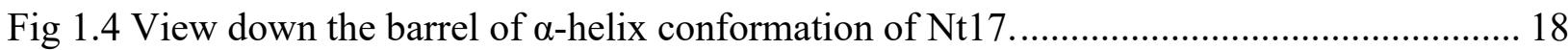

Fig 2.1 Time resolved in situ AFM images of a TBLE bilayer forming .................................. 39

Fig 2.2 Western blot analysis of the SUMOylation of httexon1(46Q)................................... 42

Fig 2.3 ThT analysis of unmodified and SUMOylated httexon1(46Q) aggregation.................. 43

Fig 2.4 Ex situ AFM images of unmodified and SUMOylated htt-exon1(46Q)...................... 44

Fig 2.5 Ex situ AFM images of unmodified htt-exon1(46Q) incubated with free SUMO1........ 46

Fig 2.6 Analysis of oligomers observed in AFM images of SUMOylated htt-exon1(46Q)........ 47

Fig 2.7Analysis of fibrils and amorphous aggregates observed in ex situ AFM images of unmodified and SUMOylated htt-exon1(46Q)...................................................... 48

Fig 2.8 Representative AFM images demonstrating the different morphologies...................... 50

Fig 2.9 CD spectral analysis of secondary structural elements of aggregates of unmodified and

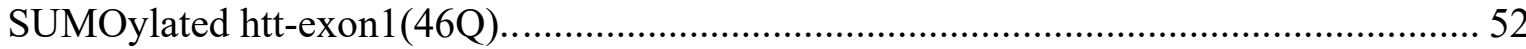

Fig 2.10 Immunochemical analysis aggregates formed by SUMOylated htt-exon1(46Q).. ....... 53

Fig 2.11 SUMOylation of htt-exon1(46Q) inhibits the ability of htt to interact with lipid......... 57

Fig 2.12 Analysis of the RMS roughness of TBLE bilayer in situ AFM images exposed to

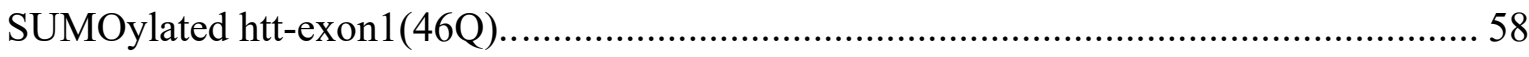

Fig 2.13 Cartoon summarizing the impact of SUMOylation on htt.................................... 61

Fig 3.1 Ex situ AFM images of htt-exon1(46Q) aggregates present after 1, 3, 5, or $8 \mathrm{~h}$...........83

Fig 3.2 Ex situ AFM images of different preparations of htt-exon1(46Q) aggregates............... 86

Fig 3.3 ThT assay tracking aggregation of htt crosslinked.................................................... 88 
Fig 3.4 Comparison of aggregate diameter measured by DLS for non-crosslinked and crosslinked htt. 89

Fig 3.5 The relative lipid binding affinity of htt oligomers without and with varying treatments of DFDNB crosslinking. 91

Fig 3.6 Comparison of the morphology of supported TBLE bilayers exposed to oligomers, fibrils, or DFDNB stabilized oligomers.

Fig 3.7 MTT toxicity assay for the N2a cells exposed to oligomers and crosslinked oligomers . 96

Fig 3.8 The proposed mechanism for interaction of oligomers and crosslinked oligomers with lipid membrane. 101

Fig 4.1 AFM images of a variety of aggregates formed by htt-exon1 proteins......................... 112

Fig 4.2 Toxic gain of function pathogenic mechanisms associated with polyQ diseases. 115 


\section{List of tables}

Table1.1 CAG repeat disorders .................................................... 


\section{List of Abbreviations}

AFM

CD

$\mathrm{CR} \%$

DFDNB

E. coli

GST

HD

$h t t$

htt-exon1(46Q)

IPTG

$\mathrm{KDa}$

Nt17

PolyQ

PTMs

PDA

RMS

TBLE

ThT

Tris
Atomic force microscopy

Circular dichroism

The percentage of colorimetric response

1,5-difluoro-2,4-dinitrobenzene

Escherichia Coli

Glutathione S-transferases

Huntington Disease

Huntingtin

Huntingtin exon 1 containing 46 Glutamines

Isopropyl $\beta$-D-1-thiogalactopyranoside

Kilo Dalton

The first $17 \mathrm{~N}$-terminal amino acids of huntingtin

Polyglutamine

Post translational modifications

Polydiacetylene

Root mean square

Total brain lipid extract

Thioflavin $\mathrm{T}$

Tris(hydroxymethyl)aminomethane 


\section{Protein aggregation and Huntington's disease}

\subsection{Neurodegenerative diseases}

Neurodegeneration refers to the progressive dysfunction and atrophy of neurons. The presence and development of abnormal protein assemblies as various intracellular proteinaceous inclusions and extracellular neurotic plaques are the most predominant features of neurodegenerative diseases. These deposits impact several areas of the brain, causing memory and cognitive impairment. Current therapeutics alleviate the symptoms of neurodegenerative diseases and developing effective therapies has been impeded by the limitation of our mechanistic-based knowledge of the underlying cause and progression of neurodegeneration.

\subsection{Amyloid formation in neurodegenerative disease}

Several age-related neurodegenerative diseases, including Alzheimer's disease (AD), Parkinson's (PD), Huntington's diseases (HD), are associated with the accumulation of micron sized insoluble proteinaceous deposits in cells and tissues. ${ }^{1-3}$ These deposits are comprised of a specific protein (or proteins) associated specifically with each disease. For example, extracellular plaques of A $\beta$ and tangles of tau are connected to (AD); Lewy bodies of $\alpha$-synuclein are implicated in (PD); and nuclear inclusions of a mutant huntingtin (htt) protein are observed in HD. These deposits are comprised of elongated filaments termed amyloid, which exhibit a cross $\beta$-sheet structure. ${ }^{4,5}$ While amyloid fibrils share this generic $\beta$-sheet ultrastructure, a variety of morphologically distinct fibrils can form, which is referred to as polymorphism. ${ }^{6-8}$ Beyond the formation of fibrillar aggregates, numerous other aggregate species, some of which are intermediates to fibrils, also form. These include small oligomers (dimers, trimers, dodecamers), high molecular weight oligomers, protofibrils, amorphous aggregates, and annular aggregates. The large variety of aggregate species 
involved in the aggregation process has made it challenging to assign toxic functions to specific aggregate structures, and identifying potent toxic species continues to be a major goal.

\subsection{Polyglutamine disorders}

A subset of protein aggregation related neurodegenerative diseases are the CAG repeat or polyglutamine (polyQ) disorders. These genetic neurodegenerative disorders are caused by an expansion of CAG repeats coding for glutamine, which leads to an expanded polyglutamine tract in the translated protein. ${ }^{12,13} \mathrm{~A}$ family of at least ten heritable polyQ disorders has been identified, (Table 1.1) including Huntington's disease (HD), the spinocerebellar ataxias, type 1 (SCA-1), type 2 (SCA-2), type 3 (SCA-3, also known as Machado-Joseph disease), type 6 (SCA-6), type 7 (SCA7), Dentatorubropalli-doluysian atrophy (DRPLA) and spinobulbar muscular atrophy (SBMA). ${ }^{14-}$

${ }^{21} \mathrm{HD}$ is the most common genetic neurodegenerative disease. While these diseases share some common pathological features, the causative genes associated with each subtype are structurally and functionally distinct. ${ }^{22,23}$ The expressed mutant proteins containing expanded polyQ ultimately lead to the degeneration of specific neuronal subpopulation in the central nervous system (CNS). For each disorder, there is a critical threshold for the length of the polyQ, which once exceeded, is directly correlated with the age of pathological onset and severity of the disease. ${ }^{24-27}$

Proteins containing expanded polyQ tract aggregate into detergent-insoluble amyloid-like structure. Studies of cellular model of polyQ diseases have revealed correlation between the polyQ length and the rate of aggregation. ${ }^{28,29}$ This aggregation results in the prominent, pathological feature associated with polyQ diseases, micron size proteinaceous inclusions. ${ }^{4,5}$ While the toxicity is mostly dependent on the length of the polyQ and protein expression level, the exact correlation of protein aggregates and the pathologies is largely unknown. The similarities in the pathogenic 
process and several shared features of protein aggregation at the cellular and molecular level indicate a common toxic gain of function for polyQ-containing proteins.

Table 1. CAG repeat disorders

\begin{tabular}{|c|c|c|c|}
\hline disease & common name & protein & polyQ \\
\hline Huntington disease (HD) & & huntingtin & $36-100$ \\
\hline spinobulbar muscular atrophy (SBMA) & Kennedy's disease & $\begin{array}{l}\text { androgen } \\
\text { receptor }\end{array}$ & $38-65$ \\
\hline $\begin{array}{l}\text { dentatorubral-pallidoluysian } \quad \text { atrophy } \\
\text { (DRPLA) }\end{array}$ & $\begin{array}{l}\text { Haw } \\
\text { syndrome }\end{array}$ & atrophin-1 & $49-88$ \\
\hline spinocerebellar ataxia type 1 (SCA1) & & ataxin-1 & $39-88$ \\
\hline spinocerebellar ataxia type 2 (SCA2) & & ataxin-2 & $33-77$ \\
\hline spinocerebellar ataxia type 3 (SCA3) & Machado-Joseph & ataxin-3 & $55-86$ \\
\hline spinocerebellar ataxia type 6 (SCA6) & & CACNA1A & $21-33$ \\
\hline spinocerebellar ataxia type 7 (SCA7) & & ataxin-7 & $38-120$ \\
\hline spinocerebellar ataxia type 12 (SCA12) & & PPP2R2B & $66-78$ \\
\hline spinocerebellar ataxia type 17 (SCA17) & & $\begin{array}{l}\text { TATA-box } \\
\text { binding protein }\end{array}$ & $47-63$ \\
\hline
\end{tabular}

Given the biological and clinical overlap among polyQ disorders (and more broadly neurodegenerative diseases in general), the therapeutic-based knowledge acquired by a given polyQ disorder is informative and possibly applicable to others. So far, there are no diseasemodifying therapies available for polyQ disorders. A current strategy under investigation has focused on lowering the expression of the target gene at the mRNA level, thereby effectively reducing the level of mutant polyQ related protein. ${ }^{30-33}$ There is also significant effort into understanding the biochemical and biophysical aspects of aggregation of polyQ-containing proteins to understand the underlying mode of toxicity in the hope that this will reveal therapeutic 
targets. High profile clinical trials that target protein aggregation associated with other neurodegenerative diseases like AD have failed ${ }^{34}$ however, these trials suffered from the necessity to enroll late stage patient as early diagnosis is not possible in most neurodegenerative disease. Early intervention may be vital for these strategies to be effective. As polyQ disorders are genetic, early diagnosis is possible, making early intervention possible. As a result, research into polyQ disorders can lead to better clinical trials to test the effectiveness of therapeutic strategies aimed at protein aggregation.

\subsection{Huntingtin protein (htt) associated with Huntington's disease (HD)}

HD is an autosomal dominant polyQ disorder characterized by involuntary choreiform movement, psychiatric manifestation, and progressive dementia. ${ }^{35}$ The neuropathological change of HD includes significant neuronal death within the striatum and subcortical brain structure, and to a lesser extent within the cortex. ${ }^{36}$ The genetic defect causing HD is located in chromosome 4, containing substantially increased trinucleotide repeats. ${ }^{21}$ The polyQ length for individuals with normal alleles is shorter than 35; however, 35-39 repeats may induce the phenotypic symptoms, 40-60 polyQ repeats results in adult onset, and repeats longer than 60 is associated with the juvenile type of HD. ${ }^{18,26,27}$

The full length of huntingtin protein (htt) contains 3144 amino acids, resulting in an approximately $350 \mathrm{kDa}$ protein. htt is a multifunctional protein playing a critical role in the regulation of gene expression, ${ }^{37}$ vesicle transport, ${ }^{38}$ and autophagy. ${ }^{39}$ The first exon of htt (httexon1) contains the polyQ domain, and this fragment (and other similar N-terminal fragments) are acutely toxic and recapitulate HD phenotype when expressed in transgenic HD animal models. ${ }^{4,40-}$

${ }^{43}$ As a result, htt-exon1 is the most actively studied htt fragment. Expression of htt-exon1 with an expanded polyQ domain is sufficient to develop a broad spectrum of phenotypic symptoms in 
transgenic mice. ${ }^{45,46}$ Furthermore, staining of postmortem HD patient's brain tissue reveals the presence of nucleation bodies colocalized with N-terminal fragment of mutant htt. ${ }^{47}$ In htt-exon1, the polyQ domain is flanked by the 17-residue long $\mathrm{N}$-terminal domain (Nt17) and a proline-rich domain (PRD) on its $\mathrm{C}$ terminal side that contains several polyproline (polyP) stretches (Fig 1.1). The primary hallmark of HD is the pronounced accumulation of mutant htt containing expanded polyQ into neuronal cytosolic or nuclear inclusions. ${ }^{4}$

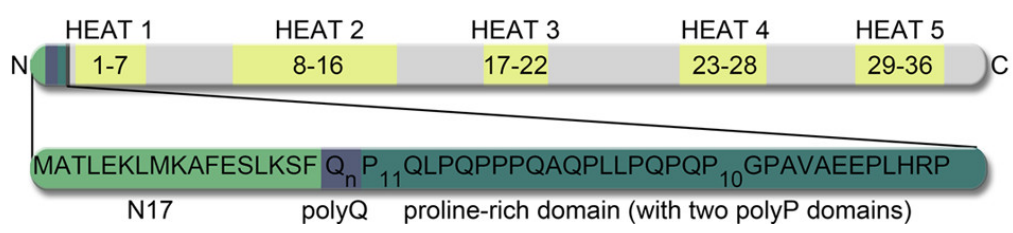

Fig 1.1 The full-length htt protein contains several HEAT repeats. The inset indicates the location of htt exon1, with the Nt17, polyQ, and proline-rich domains indicated.

\subsection{Huntingtin forms a variety of aggregate species}

While extensive research has been conducted toward understanding htt aggregation and related toxic gain of function, the precise aggregates involved in HD remains controversial. Nterminal mutant htt fragments with expanded polyQ aggregate into detergent-insoluble amyloid fibrils. ${ }^{4,48-53}$ Numerous studies using a variety of experimental systems (purified proteins to cellular and animal models) established a direct correlation between polyQ length and the rate/extent of htt aggregation. ${ }^{54-56}$ Within a cellular context, htt with longer polyQ tracts form nuclear inclusion more efficiently than htt with shorter polyQ. ${ }^{55}$ These observations are consistent with in vitro studies demonstrating that aggregation rates of mutant htt increase with further expansion of the polyQ tract. ${ }^{48,51,54}$ While mutant htt generally aggregates into fibrils, ${ }^{57-59}$ it also forms several other distinct aggregate species, including dimer, ${ }^{60}$ tetramer, ${ }^{61}$ spherical or annular oligomers $^{54,58,62}$ as well as amorphous aggregates. Biophysical and biochemical approaches have been employed to ascertain which of these aggregate species are on and off the fibril formation 
pathway. The presence of heterogeneous mixtures of distinct aggregate species make it difficult to assign toxic functions to specific aggregate structures.

Fibril formation is generally accepted to form via a nucleus-dependent mechanism. ${ }^{9-11}$ (Fig 1.2) The reaction initiates with a lag phase, which is the time required for the formation of a monomeric or multimeric critical nucleus. Once the nucleus is formed, the reaction is followed by an elongation or growth phase characterized by a relatively rapid extension of fibril aggregation. The steep transition step proceeds to a flat region known as the plateau phase and represents a steady state of the overall conversion of monomers to fibrils. The rate of aggregation is dependent on the time required for the formation of a monomeric or multimeric critical nucleus during the lag phase. A variety of factors could cause the lag phase to be shortened or ultimately eliminated. The addition of preformed fibrillar species to a sample protein under the aggregation condition (referred to as seeding process), change in the experimental condition, modification, and mutation causing the situation wherein nucleation is no longer a rate-limiting step.

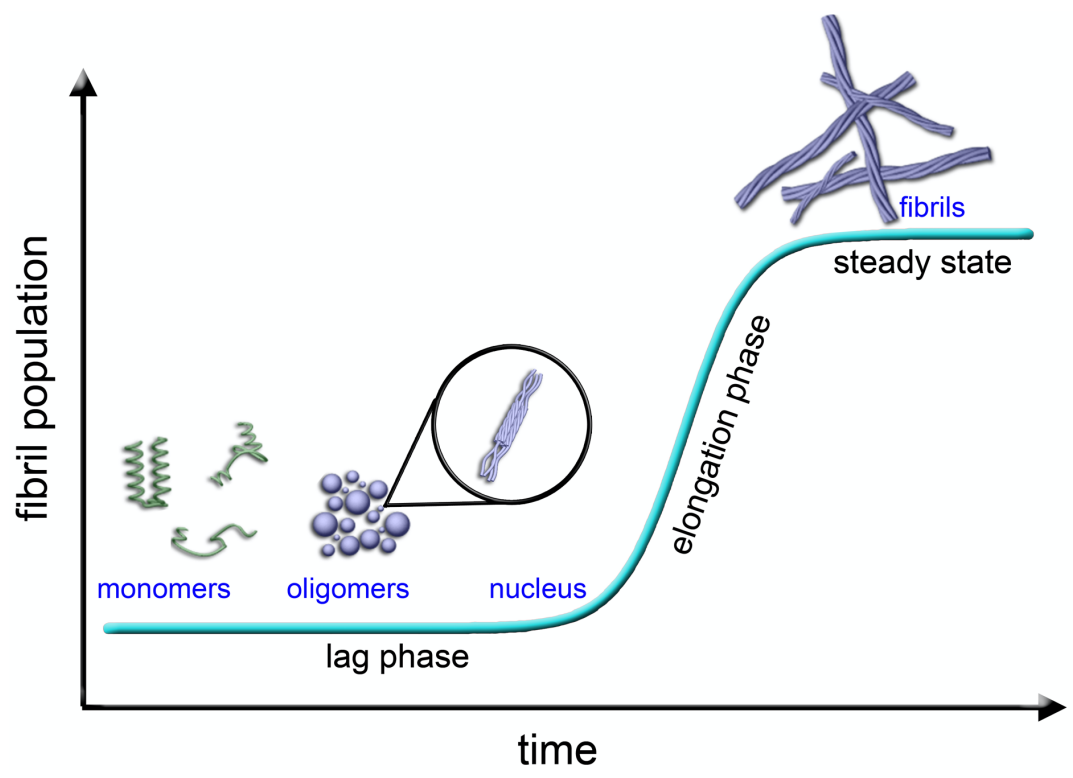

Fig 1.2 Schematic representation of the amyloid aggregation via nucleation-dependent polymerization. Lag phase involves transition from non-native protein monomers to prefibrillar oligomers, eventually forming a critical nucleus. Upon nucleation, rapid fibrils growth (elongation phase) is observed. The plateau phase represents the steady state when maximum fibrils growth has been reached. 
Despite the complexity of the aggregation mechanism, a wealth of critical biophysical information has been accumulated. Several aggregation schemes associated with the formation of fibrils in polyQ containing proteins have been proposed (Fig 1.3). Two of the more prominent one is, 1) rearrangement of monomers into structures that directly nucleate fibrils formation ${ }^{11,48,49}$ (Fig $1.3 \mathrm{~A}), 2$ ) formation of insoluble oligomeric intermediates that can associate into the $\beta$-sheet rich multimeric nucleus via structural rearrangement leading to the formation of fibrils ${ }^{54,63,64}$ (Fig 1.3B). There are also several other off pathways aggregation schemes that can lead to the formation of a variety of oligomers, annular or amorphous aggregates (Fig 1.3C). Eventually, all these higher order aggregates may accumulate together to form micron-sized inclusion that is the hallmark of htt aggregation (Fig 1.3D).

Nucleation kinetic analysis of various polyQ peptides revealed a monomeric nucleation aggregation model for the longer polyQ, while shorter polyQ length appears to aggregates via a tetrameric critical nucleus. ${ }^{11}$ Adding to the complexity of the aggregation mechanism is the detection of transient oligomers prior to fibril elongation, suggesting that both aggregation pathways could occur simultaneously. Using AFM imaging techniques, oligomers formed by various lengths of polyQ with different levels of stabilities were detected. ${ }^{54}$ Different environmental factors may also affect the appearance of oligomeric species, such as the presence of a particular aggregation surface or usage of a specific solvent involved in preparation protocols. For example, on the mica surface, oligomeric-mediated fibril formation appeared to be the dominant aggregation scheme. The intervention of the environmental factor in the fibril formation pathway points to the importance of designing experiments to mechanistically investigate aggregation in relevant cellular environment. 


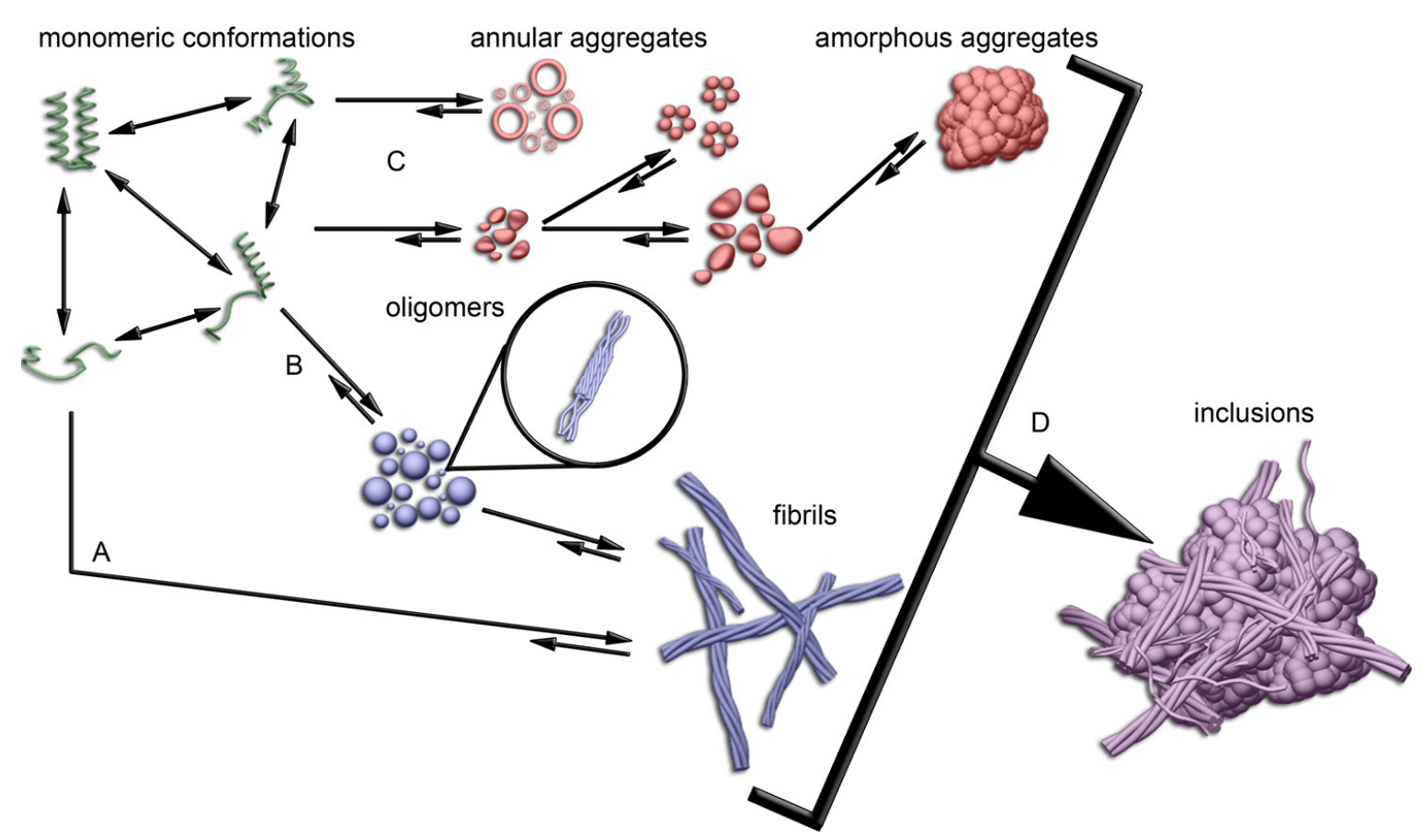

Fig 1.3 A schematic model for misfolding and aggregation of mutant htt protein. (A) A monomeric critical nucleus that leads directly to fibril formation (B) Fibril formation via oligomeric pathway (C) Off pathway aggregation routes (D) Inclusion body formation

\subsection{Ambiguity in the toxicity associated with variety of mutant htt species}

\subsubsection{Protective role of Inclusion bodies}

The precise role of different htt aggregates with regard to toxicity is still not clear. Immunostaining of postmortem brain tissues of HD patients indicated htt localization to the neuronal intranuclear and dystrophic neurite inclusions in both cortex and striatum. ${ }^{47}$ The micronsize cytoplastic and intranuclear inclusions have been reported to underlie the neuropathology observed in transgenic mouse model of HD. ${ }^{4}$ However, several observations suggested that the sequestration of mutant htt into prominent inclusion bodies is a cell-protective phenomenon. ${ }^{28,65}$ Despite the large scale of the striatum compartments being affected in the HD brain, the distribution of nuclear aggregates associated with mutant htt in the striatum does not correspond to the pathology of HD. ${ }^{66}$ Whereas neuropil aggregates, with the less common occurrence, may be involved in dendritic pathology. ${ }^{66}$ 
In cellular models of $\mathrm{HD}$, the presence of inclusion in the nucleus do not correlate with htt induced neurodegeneration. ${ }^{28}$ Toxic species might in fact exist in the diffuse fraction of mutant htt rather than inclusion bodies. ${ }^{28,65}$ That is, the dispersed fraction observed in cells may consist of toxic smaller aggregates such as fibrils and oligomers that are invisible to conventional light microscopy. The Identification and characterization of species that best predict the neuronal death within the diffused fraction of mutant htt in neurons require novel experimental techniques with high resolution to visualize species smaller than micron size inclusions. Additionally, approaches capable of distinguishing the complex mixtures of aggregates in the cell and correlating this information to the pathological outcome needs to be applied. The conflict associated with neurotoxicity of inclusions may emerge from the variety of model systems being examined, ranging from cultured cells to intact animals or postmortem tissue of HD brain patients. It also appears that the location that the inclusions have been found throughout the cells may affect the putative risk of neurotoxicity. Depending on the system of study and the locations that inclusions are found, they may cause different neurological effects. It is also possible that htt toxicity is multifaceted. That is, different aggregate species may be involved with distinct toxic mechanisms that are not mutually exclusive. Obtaining toxic assignments to each htt aggregate form could potentially provide understanding to the striking cell specificity of htt-mediated toxicity. Identification of mutant htt features that drive the emergence of fibrils, oligomers, and other small interties, as well as the subsequent molecular event triggering the initial phases of cellular toxicity may lead to novel therapeutic strategies. As a result, the factors that modulate htt aggregation toward specific aggregate species along with their association with HD pathology have been of interest. 


\subsubsection{Fibril-associated toxicity}

Due to the current ambiguity regarding the toxic species associated with htt mutant aggregates, a variety of sensitive techniques like single molecule super resolution fluorescent microscopy have recently been applied to investigate the smaller mutant htt aggregates within the diffused population. In the cytosolic compartment, small fibrils resembled those fibrils bundles detected in vitro were observed that may be linked to neurotoxicity. ${ }^{67,68}$ A combination of EM and immunoreactivity technique has identified fibrillar-like structures in both cell model and HD transgenic mice, ${ }^{4,51,69}$ as well as postmortem brain tissues. ${ }^{47}$ Interestingly, these fibrillar structures are not present in the process of inclusion body formation; rather their emergence occurs at the later stage, coinciding with the presence of mature inclusions. ${ }^{57}$ This suggests that inclusion bodies initially form from monomeric and small oligomeric htt species.

In terms of toxicity, fibrillar assembly of the htt fragment corresponding to htt-exon1 trigger apoptotic cell death. ${ }^{70}$ Even when mammalian cells are exposed to pre-formed amyloid fibrils generated by PolyQ peptides and htt-exon1 exhibited pronounced toxicity. Consistent with this, $\beta$-sheet rich fibrils of highly aggregate-competent mutant htt-exon 1 comprising of a short polyQ length are potently toxic in cell culture, and Drosophila models suggest that the underlying amyloid structure is key to some toxic mechanism. ${ }^{74}$

\subsubsection{Oligomer-associated toxicity}

Spherical oligomers were initially identified as precursor species in fibril formation associated with htt-exon1. In the past decades, identification, isolation, and characterization of oligomeric species has been of interest. Numerous studies have implied that soluble oligomers, rather than mature fibrils, may represent the primary toxic agent in HD. Based on fluorescence correlation spectroscopy, oligomers represent early forming aggregate species in cell culture. ${ }^{75}$ 
Using a combination of microscopic techniques and immunochemical assays, the formation of oligomers was confirmed to occur in the brains of the R6/2 and HdhQ150 knock-in mouse model. ${ }^{76}$ In addition, oligomers from these mouse models were biochemically and morphologically indistinguishable from htt oligomers formed in vitro from purified protein. Further studies with the HdhQ150 mouse model revealed an increase in the soluble pool of htt oligomers within the first 2 months of life, but this oligomer pool declines with age as the formation of insoluble inclusions occurs. ${ }^{77}$ This may be due to the incorporation of oligomers into inclusions. Using a number and brightness method to analyze confocal laser microscopy images, oligomers of GFPfused htt-exon1 formed on-pathway to inclusion body formation, ${ }^{78}$ and inclusions contain a heterogeneous mixture of oligomers based on EM experiments and immune-labeling. ${ }^{54} \mathrm{Htt}$ dimers and other larger multimers have been isolated from insects and mammalian cells. ${ }^{79}$

Evidence supporting a toxic role of oligomers is multifaceted. Using FCS, the formation of htt-exon1 dimers and tetramers was identified as the first marker of the cell pathology ${ }^{80}$ Early nuclear DNA damage with cell models expressing mutant htt-exon1 occurs concurrently with the formation of diffusible aggregates (oligomers), while sedimentable aggregates (fibrils) only appear at later time points. Overexpression of profilin, an abundant cellular protein that preferentially binds to oligomers, reduces htt toxicity, supporting a toxic role for oligomers. ${ }^{81}$ Oligomers are also linked to ER stress that occurs prior to visible inclusion formation. ${ }^{82-84}$

It may be true that both soluble and insoluble species engender neurotoxicity, albeit through different mechanisms. For example, soluble htt oligomers may promote cell death via apoptosis induced by mitochondrial dysfunction, whereas, insoluble aggregates cause coaggregation of essential cellular machinery, resulting in slower cell death via necrosis. ${ }^{85}$ This hypothesis argues against the notion that a single aggregate species is toxic. More discrepancies in 
assigning toxicity to a specific molecular state originate from continuous production of htt monomers that fuel the aggregation process, multiple intermediate stages, and lack of precision in the microscopic techniques to distinguish between these species. Further studies to narrow the molecular states during early toxic events are critically important in understanding HD and its pathology.

\subsection{The role of $\mathrm{Nt17}$ nucleating aggregation}

Although htt aggregation is polyQ length dependent, the protein context of the polyQ tract strongly influences aggregation and toxicity. Of particular interest is Nt17, which impacts polyQ structure, aggregation propensity, and aggregate stability. Several aggregation mechanisms appear to be mediated by Nt17. Specifically, Nt17 is implicated in driving the initial stages of htt aggregation via oligomerization. ${ }^{86-89} \mathrm{Nt} 17$ is predominantly a disordered random coil in solution, ${ }^{90,91}$ but it undergoes a conformational transitions to adopt an amphipathic $\alpha$-helical structure when exposed to binding partners. ${ }^{64,90,92-94}$ This propensity to form an amphipathic $\alpha-$ helix leads to intermolecular association of $\mathrm{Nt} 17$ domains to form $\alpha$-helix rich oligomers that lower the energy barrier to nucleation by bringing polyQ tracts into close proximity. ${ }^{60,64,80,90,95,96}$ Interestingly, the influence of Nt17 on the aggregation of PolyQ peptide is independent of the position, as Nt17 incorporated to either N-terminal and C-terminal sides of PolyQ peptides promote the aggregation. ${ }^{64}$ Similarly, htt-exon1 fragments lacking Nt17 aggregate much more slowly than full length htt-exon $1 .{ }^{97}$ These peptide models imply the necessity of the homotypic association of $\mathrm{Nt17}$ as a key to multimeric nucleus formation and thereby offering Nt17 as an intrinsic enhancer to htt aggregation.

By probing sporadically populated oligomer species with atomic resolution by NMR, a branching pathway for the initial multimerization event of $\mathrm{Nt} 17$ has been recently proposed. ${ }^{60} \mathrm{In}$ 
this model, the hydrophobic interaction between two helices drives the formation of two types of antiparallel dimeric subunits, one classified as non-productive (incapable of further oligomerization), and the other being productive by assembling into a symmetric tetramer with an orthogonal orientation of two dimers. The model provides further support for the amphipathic characteristics of helices as hydrophilic residues within tetrameric units are solvent exposed, and available to further electrostatic and amphipathic interaction. The tetrameric subunit is compatible with the notion that oligomerization localizes PolyQ tracts into close proximity and promoting fibril nucleation. Nt17 mostly retains helical structure in fibrils. ${ }^{98}$ However, this $\alpha$-helical structure does not necessarily propagate along the entire length of $\mathrm{Nt17}$. The $\mathrm{C}$-terminal region may remain disordered in the fibril structure, providing flexibility for structural transition to the antiparallel $\beta$ sheet rich fibril core. ${ }^{87,95,99}$

Nt17 may also be directly involved in intermolecular interaction with polyQ that promote amyloidogenic conformers. ${ }^{97}$ That is, interactions between polyQ and Nt17 may stimulate conversion within the polyQ region to aggregation-prone conformation. Co-incubation of Nt17 peptides (no polyQ domain) with pure polyQ peptides (no Nt17) enhances aggregation, supporting the notion of a strong intermolecular interaction between $\mathrm{Nt} 17$ and polyQ. In similar experiments, incubating Nt17 peptide (no polyQ domain) with htt-mimicking peptides containing Nt17 domain suppressed aggregation. The free Nt17 peptides are thought to incorporate into htt oligomers that are initially formed by intermolecular association of Nt17 domains. The incorporated free Nt17 peptides effectively act as spacers, increasing the distance between polyQ domains and suppressing nucleation. ${ }^{89}$

Given the substantial contribution of $\mathrm{Nt17}$ in htt aggregation, targeting this domain as a htt aggregation modulator has been subject to an active investigation. Removing N17 via cleavage ${ }^{11}$ 
and targeting N17 directly via molecular chaperones ${ }^{97}$ or antibodies are common strategies. ${ }^{100}$ Removal of Nt17 leads to a peptide with a significantly decreased aggregation kinetics. ${ }^{11}$ The chaperonin TRiC, blocking the hydrophobic face of the Nt17 helix, renders a dominant negative effect on htt aggregation in vitro and in vivo. ${ }^{97}$ The Hsc 70 chaperon also binds Nt17, competing with the homotypic interaction between $\mathrm{Nt} 17$ domains that trigger the aggregation process. ${ }^{101}$ Moreover, Introducing prolines as helix-breaking (HB) or polyalanine residues to the Nt17 tract has been shown to block the aggregation of htt, indicating the specific characteristic of Nt17 in the stability of the aggregated htt conformation. ${ }^{97}$ As a result, Nt17 appears to be a viable therapeutic target. Importantly, due to Nt17's role in initiating oligomer formation that leads to fibrillization, targeting it could prevent the formation of both types of htt aggregates.

\subsection{Htt membrane localization is implicated in toxic mechanism}

A complicating cellular factor in unraveling mutant htt toxicity is the modifying impact of association with a great number of membranous surfaces. Htt is significantly associated with membranous organelles, including mitochondria, endoplasmic reticulum (ER), the nuclear envelope, tubulovesicles, endosomes, lysosome, and synaptic vesicles. ${ }^{47,66,83,102,103}$ These observations indicate a strong htt/lipid binding interaction that may be related to the physiological and pathological role of htt.

The strong htt-lipid interaction is demonstrated by studies in cellular and mouse model as well as HD brain human cells. Membrane compartments are incorporated onto the surfaces of htt inclusion bodies in cellular models. ${ }^{4,104,105}$ Inclusions formed in HD mouse models and immortalized human cells directly interact with cellular endomembrane that implicated in cellular damage. ${ }^{106,107}$ The ability of htt to bind membranes are potentially modified by aggregation state, and distinct aggregation pathways may be induced on a membrane surface. Supporting the first 
notion, htt oligomers localize to the postsynaptic membrane involved in membrane trafficking and several other neurological features of $\mathrm{HD}$ in $\mathrm{R} 6 / 2$ mice. ${ }^{106} \mathrm{Htt}$ associate with the outer mitochondrial membrane and mitochondrial structural proteins, leading to mitochondrial fragmentation, abnormal mitochondrial dynamics, and oxidative DNA damage. ${ }^{108}$ While oligomers may transiently exist in the bulk solution due to the intermediated nature, subcellular localization of oligomers can still promote future aggregation or even stabilize the oligomers that lead to cytotoxicity.

With the localization of htt to membranes being established, unraveling the mechanistic details of htt/lipid interactions are of great interest. It is worth noting that lipid membranes, providing a 2-dimensional scaffold, are a common modulator of amyloid formation. ${ }^{109}$ Several physiological properties of the lipid membrane, such as fluidity, curvature, elasticity, modulus, surface charge, alter the aggregation of amyloid protein. ${ }^{109,110}$ PolyQ peptides and htt-exon1 all demonstrate altered aggregation in the presence of membranes compared to the bulk solution. ${ }^{88,111-}$ ${ }^{113}$ Using a supported lipid membrane model, polyQ length-dependent interaction of htt-exon1 and synthetic peptide with lipid membrane was indicated that resulted in altered htt aggregation and disruption of the membrane integrity. ${ }^{111} \mathrm{Htt}$ association and aggregation on lipid membranes alter the local mechanical properties of the lipid membrane, leading to membrane permeabilization..$^{88,112,114,115}$ As htt interaction and aggregation at lipid surfaces represent a fundamental step in several pathogenic mechanisms associated with HD, understanding a detailed understanding of the interaction of htt with cellular and subcellular surface is significant. Moreover, blocking the ability of htt to directly bind to lipid membranes represents a novel therapeutic strategy for HD. There are many reports of membrane permeabilization caused by 
mutant htt with expanded polyQ; ${ }^{88,114}$ however, it is not clear whether these effects are specific to fibrillar assembly nor whether they are common to other types of spices like oligomers.

\subsection{Nt17 Function as a lipid binding domain}

As previously mentioned, $\mathrm{Nt17}$ is a random coil in aqueous solution that can adopt an amphipathic $\alpha$-helical conformation which associates with lipid membranes..$^{91,92,116} \mathrm{Nt} 17$ mediates trafficking of htt-exon1 to membranes associated with ER, mitochondria, and Goli, contributing to mutant htt-induced disruption of cellular calcium homeostasis. ${ }^{92,117} \mathrm{Nt17}$ is essential for insertion of htt into a variety model of lipid bilayers, lending support to the notion that Nt17 plays a key role in htt function and toxicity. ${ }^{88,118}$

Based on molecular dynamics, a mechanism for Nt17 lipid binding has been proposed. ${ }^{116,119,120}$ In this mechanism, membrane anchoring is initially driven by electrostatic interaction between hydrophilic residues of Nt17 and the lipid headgroups. Next, Nt17 structurally reorganizes to position nonpolar residues near the bilayer. Ultimately, nonpolar residue partition inside the hydrophobic core of the membrane with a nearly parallel alignment as $\mathrm{Nt} 17$ competes adoption of its $\alpha$-helical structure. Furthermore, Nt17 membrane association provides a scaffold that promotes intermolecular $\mathrm{Nt17}$ interactions, inducing multimerization on the membrane surface. Thereby, Nt17 localization with lipid membrane can produce a high local concentration of polyQ domains that may trigger nucleation and facilitate aggregation; however, this appears to be dependent on the lipid composition.

\subsection{Importance of lysine residues in $\mathrm{Nt17}$}

As the importance of $\mathrm{Nt17}$ in htt aggregation has been well established, efforts have been put forth to understand the structural details of the role specific residues in Nt17 play in mediating aggregation and toxicity. Of particular interest are the three lysine residues within Nt17, as lysine 
has the ability to participate in both hydrophobic and electrostatic interactions, both of which often play prominent roles as mediators in aggregation. In many cases, disrupting interactions associated with lysine resides significantly interferes with amyloid formation. ${ }^{121-125}$

The lysine residues within Nt17 reside at positions 6, 9, and 15 (K6, K9, and K15). These residues appear to play a vital role stabilizing htt oligomers ${ }^{95}$ and lipid binding. ${ }^{126}$ Of the three lysine residues, $\mathrm{K} 6$ and $\mathrm{K} 15$ are located at the edge of the hydrophilic/hydrophobic interface in the amphipathic helix, giving them the potential to participate in stabilizing salt-bridges when Nt17 domains self-assemble into $\alpha$-helix rich oligomers. K6 and K15 undergo significantly less deuterium back-exchange in aggregated forms of huntingtin. ${ }^{127}$ Deuterium protection of these buried residues is indicative of structural protection, supporting contributions of K6 and K15 in intermolecular interactions in the aggregated state. Rapid back exchange of K9 suggests that it remains accessible, which is consistent with its location in the amphipathic $\alpha$-helix. ${ }^{127}$ These observations of the relative importance of these different lysine residues in stabilizing oligomers is consistent with a more recently reported tetrameric structure of $\mathrm{Nt} 17 .{ }^{60}$

Lysine residues in Nt17 play a critical role in membrane binding as well. NMR-based topological analysis of the conformational ensemble of Nt17 residues in micellar environment demonstrated the parallel angle of the $\alpha$-helix axis to the lipid membrane. ${ }^{116}$ In this orientation, $\mathrm{K} 15$ is buried in the lipid structure, and $\mathrm{K} 6$ and $\mathrm{K} 9$ are more exposed to the aqueous phase. This arrangement facilitates electrostatic interactions between Nt17 domains and possibly with the proximal polyQ tract. High resolution atomistic model of Nt17 obtained by MD simulation in the presence of POPC further unveiled essential interactions between lysine residues and membranes. ${ }^{128}$ Based on this model, $\mathrm{K} 6$ and $\mathrm{K} 15$ are involved in several hydrogen bonds with the oxygen of the phosphate groups and several salt-bridges with the negatively charged 
phospholipids. Such salt bridges with high probability of occurring appear to be the critical components of the interaction of Nt17 with the bilayer. While K9 has a lower probability of forming salt-bridges with the phospholipids, contrary to the other lysines, it is often associated with intramolecular salt-bridges with other amino acids. ${ }^{128,129}$

\subsection{Post translational modification of Nt17 alter htt aggregation and lipid binding}

Another key player in htt subcellular localization, clearance, and aggregation is posttranslational modification (PTM) within Nt17. Given the critical role of Nt17 in aggregation and membrane association, considerable effort has been devoted to identifying PTMs within Nt17 and the consequence of these modifications on htt toxicity, cellular function, self-association, and lipid binding properties. ${ }^{130-134} \mathrm{Nt} 17$ domain contains various sites that undergo PTMs, including Phosphorylation, ${ }^{131}$, Acetylation, ${ }^{126}$ Ubiquitination, ${ }^{135}$ SUMOylation, Oxidation (Fig 1.4).

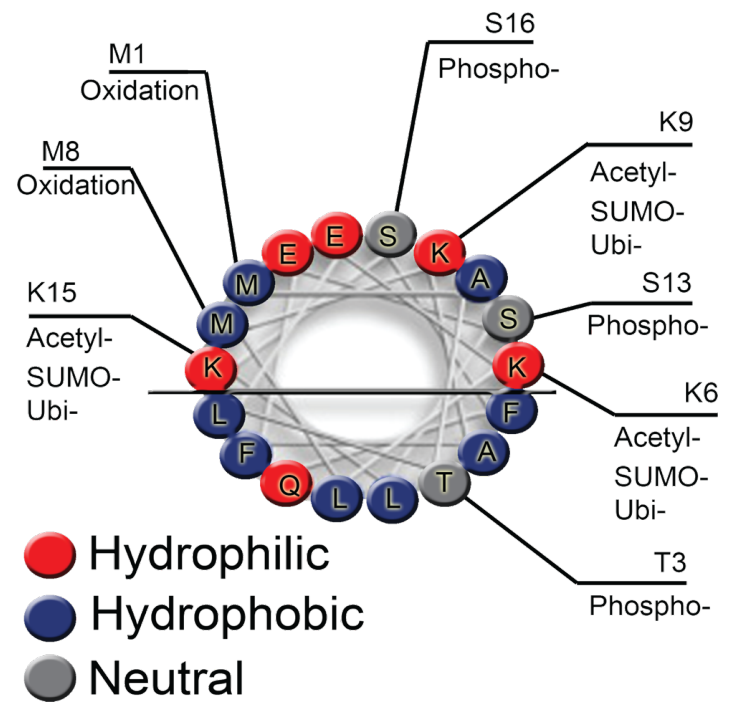

Fig 1.4 View down the barrel of $\alpha$-helix conformation of Nt17 showing relative hydrophobicity of each residue as well as the sites of reported post-translational modification. 
There are three phosphorylation sites within Nt17: Threonine 3 (T3), Serine 13 (S13), and serine 16 (S16). Phosphorylation of Nt17 reduces htt toxicity and initiates a protective response in transgenic mice. ${ }^{131,136-140}$ Introduction of phosphomimic mutation at S13 and S16 in transgenic mouse models reduce visible htt inclusions within the brain and were associated with improved phenotype. ${ }^{138}$ In vitro, these same phosphomimetic mutations within htt peptides significantly slowed aggregation and reduced structural flexibility and stability of the resulting fibrils..$^{90,141,142}$ This is consistent with the introduction of negative charges near the C-terminus of an $\alpha$-helix destabilizing its structure. The helicity disrupting effect of phosphorylation likely reduces the efficiency of forming Nt17-mediated oligomers known to be involved in nucleating fibrillization.

Lysine residues within Nt17 can undergo acetylation, SUMOylation, and ubiquitination. ${ }^{126,132,135}$ Proteomic mapping by MS demonstrated that K9 is substantially acetylated in mammalian cell lysates, ${ }^{143}$ and this residue is preferentially chemically acetylated in vitro. ${ }^{126}$ Acetylation across all three lysine residues in $\mathrm{Nt} 17$ retards fibril formation in solution and promotes larger globular aggregates. ${ }^{126}$ However, selective acetylation at an individual lysine does not impact aggregation. ${ }^{144} \mathrm{Nt17}$ acetylation also reduces the affinity of htt for lipids, limiting its ability to cause membrane disruption and ameliorate htt-induced toxicity. ${ }^{126}$ Ubiquitination of Nt17 tags htt for degradation by the ubiquitin proteasome system (UPS), reducing mutant htt toxicity in knock-in mouse model of HD. ${ }^{135}$ Competing with ubiquitination, SUMOylation of Nt17 also modifies HD pathogenesis. ${ }^{132}$ SUMOylation at K6 and K9 reduce aggregation by stabilizing htt-exon1 fragments in cultured cells, but this is associated with exacerbated toxicity in Drosophila. ${ }^{132}$ The small G protein, Rhes, which serves as a SUMO E3 ligase, preferentially binds mutant htt, inducing SUMOylation. ${ }^{145}$ Overexpressing Rhes in a striatal cell line elicited a pronounced increase in soluble mutant htt, exacerbating cytotoxicity. However, mutating Nt17 
lysine residues to arginine interferes with Rhes-induced htt SUMOylaltion, abolishing cytotoxicity.145 Another E3 SUMO ligase, PIAS1, regulates htt SUMOylation in Drosopophila models, and reducing expression of PIAS decreased accumulation of insoluble htt with an apparent effect.146 Over-expressing PIAS in the R6/2 HD mouse model of HD increases protein deposition and exasperates behavioral phenotype. ${ }^{147}$

As PTMs regulate cellular events, understanding of the pathophysiological relevance of PTMs in htt aggregation would provide details of how mutant htt affects cell function leading to neurotoxicity. Besides, identifying regulatory factors of Nt17 PTMs may provide novel therapeutic targets. In addition, elucidation of the implication of PTMs in htt aggregation is critical for understanding the context-dependent structural impact of Nt17 mediated aggregation mechanisms. Broad knowledge of the structure/function relationship of $\mathrm{Nt} 17$ can potentially lead to developing strategies to manipulate $\mathrm{Nt} 17$ function to treat $\mathrm{HD}$.

\subsection{Dissertation outline and study rational}

Although significant strides have been made in understanding HD, no therapeutic has been discovered to stop the progression of the disease. Htt aggregation has been long associated with HD. Nt17 is strongly believed to impact the aggregation of htt and function as the lipid binding domain. Varieties of strategies have been applied to regulate the propensity of Nt17 to selfassociate, and its ability to bind membranes can be regulated. PTMs within Nt17 affect both aggregation and membrane binding. Furthermore, a variety of htt aggregates are incorporated into cellular lipid membranes in cellular and animal HD model, as well as in postmortem HD brains, yet little is known about how aggregated forms of htt actively interact with cellular membranes. Based on these critical gaps in our understanding of $\mathrm{HD}$, the following topics are addressed in this dissertation: 
1. The impact of Nt17 SUMOylation on htt aggregation and membrane activity. While SUMOylation affects the accumulation of htt inclusions in cells, there is no characteristic clarification of the impact of SUMOylation on more diffuse forms of htt aggregates. Furthermore, the impact of SUMOylation on htt membrane activity is completely lacking. In Chapter 2, the direct impact of SUMOylation of $\mathrm{Nt} 17$ on htt aggregation is characterized, and SUMOylation promotes the formation of large, SDS-soluble amorphous aggregates of htt. Additionally, the influence of SUMOylation on htt membrane activity was investigated, and SUMOylation greatly inhibited the ability of htt to bind and aggregate on lipid membranes.

2. The role of aggregation state on htt's ability to interact with lipid membranes. While the association of htt with lipids is well established, there has been no biophysical investigation into how aggregation state impacts htt/membrane interactions. Chapter 3 investigates the propensity of a distinct htt aggregates to bind lipid membranes. Based on these studies, htt oligomers have the highest membrane affinity. In an attempt to specifically characterize the binding of htt oligomers to lipid membranes, a crosslinking strategy directed at lysine residues within Nt17 was employed to stabilize transient oligomers. While large doses of the crosslinking agent indeed stabilized oligomers, low doses surprisingly enhanced fibrillization. While the initial strategy was to use stabilized oligomers to understand how to interact with lipid membranes, crosslinking actually abolished their membrane activity, and this correlated with a reduction in their cytotoxicity, suggesting htt/lipid interactions as a novel therapeutic target. 


\section{References}

(1) Roizin, L.; Stellar, S.; Willson, N.; Whittier, J.; Liu, J. C. Electron Microscope and Enzyme Studies in Cerebral Biopsies of Huntington's Chorea. Trans. Am. Neurol. Assoc. 1974, 99, 240-243.

(2) Dasari, M.; Espargaro, A.; Sabate, R.; Amo, J. M. L. del; Fink, U.; Grelle, G.; Bieschke, J.; Ventura, S.; Reif, B. Bacterial Inclusion Bodies of Alzheimer's Disease $\beta$-Amyloid Peptides Can Be Employed to Study Native-Like Aggregation Intermediate States. Chem. Bio. Chem. 2011, 12 (3), 407-423.

(3) Chen, L.; Feany, M. B. $\alpha$-Synuclein Phosphorylation Controls Neurotoxicity and Inclusion Formation in a Drosophila Model of Parkinson Disease. Nat. Neurosci. 2005, 8 (5), 657-663.

(4) Davies, S. W.; Turmaine, M.; Cozens, B. A.; DiFiglia, M.; Sharp, A. H.; Ross, C. A.; Scherzinger, E.; Wanker, E. E.; Mangiarini, L.; Bates, G. P. Formation of Neuronal Intranuclear Inclusions Underlies the Neurological Dysfunction in Mice Transgenic for the HD Mutation. Cell 1997, 90 (3), 537-548.

(5) Difiglia, M.; Sapp, E.; Chase, K. O.; Davies, S. W.; Bates, G. P.; Vonsattel, J. P.; Aronin, N. Aggregation of Huntingtin in Neuronal Intranuclear Inclusions and Dystrophic Neurites in Brain. Science. 1997, 277 (5334), 1990-1993.

(6) Lin, H.-K.; Boatz, J. C.; Krabbendam, I. E.; Kodali, R.; Hou, Z.; Wetzel, R.; Dolga, A. M.; Poirier, M. A.; van der Wel, P. C. A. Fibril Polymorphism Affects Immobilized Non-Amyloid Flanking Domains of Huntingtin Exon1 Rather than Its Polyglutamine Core. Nat. Commun. 2017, 8 (1), 15462.

(7) Kodali, R.; Williams, A. D.; Chemuru, S.; Wetzel, R. Abeta(1-40) Forms Five Distinct Amyloid Structures Whose Beta-Sheet Contents and Fibril Stabilities Are Correlated. J. Mol. Biol. 2010, 401 (3), 503-517.

(8) Riek, R.; Eisenberg, D. S. The Activities of Amyloids from a Structural Perspective. Nature. 2016, 539 (7628), 227-235.

(9) Xue, W.-F.; Homans, S. W.; Radford, S. E. Systematic Analysis of Nucleation-Dependent Polymerization Reveals New Insights into the Mechanism of Amyloid Self-Assembly. P. Natl. Acad. Sci. USA. 2008, 105 (26), 8926-8931.

(10) Chiti, F.; Dobson, C. M. Protein Misfolding, Functional Amyloid, and Human Disease. Annu. Rev. Biochem. 2006, 75 (1), 333-366.

(11) Kar, K.; Jayaraman, M.; Sahoo, B.; Kodali, R.; Wetzel, R. Critical Nucleus Size for DiseaseRelated Polyglutamine Aggregation Is Repeat-Length Dependent. Nat. Struct. Mol. Biol. 2011, 18 (3), 328-336.

(12) Hegde, M. V.; Saraph, A. A. Unstable Genes Unstable Mind: Beyond the Central Dogma of Molecular Biology. Med. Hypotheses. 2011, 77 (2), 165-170.

(13) La Spada, A. R.; Taylor, J. P. Repeat Expansion Disease: Progress and Puzzles in Disease Pathogenesis. Nat. Rev. Genet. 2010, 11 (4), 247-258.

(14) Kawaguchi, Y.; Okamoto, T.; Taniwaki, M.; Aizawa, M.; Inoue, M.; Katayama, S.; Kawakami, H.; Nakamura, S.; Nishimura, M.; Akiguchi, I.; Kimura, J.; Narumiya, S.; Kakizuka, A. CAG Expansions in a Novel Gene for Machado-Joseph Disease at Chromosome 14q32.1. Nat. Genet. 1994, 8 (3), 221-228.

(15) David, G.; Abbas, N.; Stevanin, G.; Dürr, A.; Yvert, G.; Cancel, G.; Weber, C.; Imbert, G.; Saudou, F.; Antoniou, E.; Drabkin, H.; Gemmill, R.; Giunti, P.; Benomar, A.; Wood, N.; Ruberg, M.; Agid, Y.; Mandel, J.-L.; Brice, A. Cloning of the SCA7 Gene Reveals a Highly Unstable CAG Repeat Expansion. Nat. Genet. 1997, 17 (1), 65-70.

(16) Koide, R.; Kobayashi, S.; Shimohata, T.; Ikeuchi, T.; Maruyama, M.; Saito, M.; Yamada, M.; Takahashi, H.; Tsuji, S. A Neurological Disease Caused By an Expanded CAG Trinucleotide Repeat in The TATA-Binding Protein Gene: A New Polyglutamine Disease, Hum. Mol. Genet. 1999, 8 (11), 2047-2053. 
Komure, O.; Sano, A.; Nishino, N.; Yamauchi, N.; Ueno, S.; Kondoh, K.; Sano, N.; Takahashi, M.; Murayama, N.; Kondo, I.; Nagafuchi, S.; Yamada, M.; Kanazawa, I. DNA Analysis in Hereditary Dentatorubral-Pallidoluysian Atrophy: Correlation between CAG Repeat Length and Phenotypic Variation and the Molecular Basis of Anticipation. Neurology. 1995, 45 (1), 143-149. Andresen, J. M.; Gayán, J.; Djoussé, L.; Roberts, S.; Brocklebank, D.; Cherny, S. S.; Cardon, L. R.; Gusella, J. F.; MacDonald, M. E.; Myers, R. H.; Housman, D. E.; Wexler, N. S. The Relationship Between CAG Repeat Length and Age of Onset Differs for Huntington's Disease Patients with Juvenile Onset or Adult Onset. Ann. Hum. Genet. 2007, 71 (3), 295-301.

(19) Nakamura, K.; Jeong, S.-Y.; Uchihara, T.; Anno, M.; Nagashima, K.; Nagashima, T.; Ikeda, S.; Tsuji, S.; Kanazawa, I. SCA17, a Novel Autosomal Dominant Cerebellar Ataxia Caused by an Expanded Polyglutamine in TATA-Binding Protein. Hum. Mol. Genet. 2001, 10 (14), 1441-1448. Pulst, S.-M.; Santos, N.; Wang, D.; Yang, H.; Huynh, D.; Velazquez, L.; Figueroa, K. P. Spinocerebellar Ataxia Type 2: PolyQ Repeat Variation in the CACNA1A Calcium Channel Modifies Age of Onset. Brain. 2005, 128 (10), 2297-2303.

(21) MacDonald, M. E.; Ambrose, C. M.; Duyao, M. P.; Myers, R. H.; Lin, C.; Srinidhi, L.; Barnes, G.; Taylor, S. A.; James, M.; Groot, N.; MacFarlane, H.; Jenkins, B.; Anderson, M. A.; Wexler, N. S.; Gusella, J. F.; Bates, G. P.; Baxendale, S.; Hummerich, H.; Kirby, S.; North, M.; Youngman, S.; Mott, R.; Zehetner, G.; Sedlacek, Z.; Poustka, A.; Frischauf, A. M.; Lehrach, H.; Buckler, A. J.; Church, D.; Doucette-Stamm, L.; O’Donovan, M. C.; Riba-Ramirez, L.; Shah, M.; Stanton, V. P.; Strobel, S. A.; Draths, K. M.; Wales, J. L.; Dervan, P.; Housman, D. E.; Altherr, M.; Shiang, R.; Thompson, L.; Fielder, T.; Wasmuth, J. J.; Tagle, D.; Valdes, J.; Elmer, L.; Allard, M.; Castilla, L.; Swaroop, M.; Blanchard, K.; Collins, F. S.; Snell, R.; Holloway, T.; Gillespie, K.; Datson, N.; Shaw, D.; Harper, P. S. A Novel Gene Containing a Trinucleotide Repeat That Is Expanded and Unstable on Huntington's Disease Chromosomes. Cell. 1993, 72 (6), 971-983.

(22) Weber, J. J.; Sowa, A. S.; Binder, T.; Hübener, J. From Pathways to Targets: Understanding the Mechanisms behind Polyglutamine Disease. Biomed Res. Int. 2014, 2014, 1-22.

(23) Saunders, H. M.; Bottomley, S. P. Multi-Domain Misfolding: Understanding the Aggregation Pathway of Polyglutamine Proteins. Protein. Eng. Des. Sel. 2009, 22 (8), 447-451.

(24) Lieberman, A. P.; Shakkottai, V. G.; Albin, R. L. Polyglutamine Repeats in Neurodegenerative Diseases. Annu. Rev. Pathol. 2019, 14 (1), 1-27.

(25) Silva, A.; de Almeida, A. V.; Macedo-Ribeiro, S. Polyglutamine Expansion Diseases: More than Simple Repeats. J. Struct. Biol. 2018, 201 (2), 139-154.

(26) Penney, J. B.; Vonsattel, J.-P.; Macdonald, M. E.; Gusella, J. F.; Myers, R. H. CAG Repeat Number Governs the Development Rate of Pathology in Huntington's Disease. Ann. Neurol. 1997, 41 (5), 689-692.

(27) Snell, R. G.; MacMillan, J. C.; Cheadle, J. P.; Fenton, I.; Lazarou, L. P.; Davies, P.; MacDonald, M. E.; Gusella, J. F.; Harper, P. S.; Shaw, D. J. Relationship between Trinucleotide Repeat Expansion and Phenotypic Variation in Huntington's Disease. Nat. Genet. 1993, 4, 393-397.

(28) Saudou, F.; Finkbeiner, S.; Devys, D.; Greenberg, M. E. Huntingtin Acts in the Nucleus to Induce Apoptosis but Death Does Not Correlate with the Formation of Intranuclear Inclusions. Cell .1998, 95 (1), 55-56.

(29) Tanaka, M.; Morishima, I.; Akagi, T.; Hashikawa, T.; Nukina, N. Intra- and Intermolecular $\beta$ Pleated Sheet Formation in Glutamine-Repeat Inserted Myoglobin as a Model for Polyglutamine Diseases. J. Biol. Chem. 2001, 276 (48), 45470-45475.

(30) Xia, H.; Mao, Q.; Eliason, S. L.; Harper, S. Q.; Martins, I. H.; Orr, H. T.; Paulson, H. L.; Yang, L.; Kotin, R. M.; Davidson, B. L. RNAi Suppresses Polyglutamine-Induced Neurodegeneration in a Model of Spinocerebellar Ataxia. Nat. Med. 2004, 10 (8), 816-820.

(31) Scholefield, J.; Watson, L.; Smith, D.; Greenberg, J.; Wood, M. J. Allele-Specific Silencing of Mutant Ataxin-7 in SCA7 Patient-Derived Fibroblasts. Eur. J. Hum. 2014, 22 (12), 1369-1375.

(32) Gonzalez-Alegre, P. Recent Advances in Molecular Therapies for Neurological Disease: Triplet Repeat Disorders. Hum. Mol. Genet. 2019, 28 (R1), R80-R87. 
(33) Chan, H. Y. E. RNA-Mediated Pathogenic Mechanisms in Polyglutamine Diseases and Amyotrophic Lateral Sclerosis. Front. Cell. Neurosci. 2014, 8.

(34) Cummings, J. Lessons Learned from Alzheimer Disease: Clinical Trials with Negative Outcomes. Clin. Transl. Sci. 2018, 11 (2), 147-152.

(35) Martin, J. B.; Gusella, J. F. Huntingtons Disease. Lancet. 2007, 369, 218-228.

(36) Vonsattel, J.-P.; Myers, R. H.; Stevens, T. J.; Ferrante, R. J.; Bird, E. D.; Richardson, E. P. Neuropathological Classification of Huntington's Disease. J. Neuropathol. Exp. Neurol. 1985, 44 (6), 559-577.

(37) Valor, L. M. Transcription, Epigenetics and Ameliorative Strategies in Huntington's Disease: A Genome-Wide Perspective. Mol. Neurobiol. 2015, 51 (1), 406-423.

(38) Harjes, P.; Wanker, E. E. The Hunt for Huntingtin Function: Interaction Partners Tell Many Different Stories. Trends. Biochem. Sci. 2003, 28 (8), 425-433.

(39) Ochaba, J.; Lukacsovich, T.; Csikos, G.; Zheng, S.; Margulis, J.; Salazar, L.; Mao, K.; Lau, A. L.; Yeung, S. Y.; Humbert, S.; Saudou, F.; Klionsky, D. J.; Finkbeiner, S.; Zeitlin, S. O.; Marsh, J. L.; Housman, D. E.; Thompson, L. M.; Steffan, J. S. Potential Function for the Huntingtin Protein as a Scaffold for Selective Autophagy. PNAS. 2014, 111 (47), 16889-16894.

(40) Yang, S.-H.; Cheng, P.-H.; Banta, H.; Piotrowska-Nitsche, K.; Yang, J.-J.; Cheng, E. C. H.; Snyder, B.; Larkin, K.; Liu, J.; Orkin, J.; Fang, Z.-H.; Smith, Y.; Bachevalier, J.; Zola, S. M.; Li, S.-H.; Li, X.-J.; Chan, A. W. S. Towards a Transgenic Model of Huntington's Disease in a NonHuman Primate. Nature. 2008, 453 (7197), 921-924.

(41) Hoffner, G.; Island, M.-L.; Djian, P. Purification of Neuronal Inclusions of Patients with Huntington's Disease Reveals a Broad Range of N-Terminal Fragments of Expanded Huntingtin and Insoluble Polymers. J. Neurochem. 2005, 95 (1), 125-136.

(42) Wellington, C. L.; Ellerby, L. M.; Gutekunst, C.-A.; Rogers, D.; Warby, S.; Graham, R. K.; Loubser, O.; Raamsdonk, J. van; Singaraja, R.; Yang, Y.-Z.; Gafni, J.; Bredesen, D.; Hersch, S. M.; Leavitt, B. R.; Roy, S.; Nicholson, D. W.; Hayden, M. R. Caspase Cleavage of Mutant Huntingtin Precedes Neurodegeneration in Huntington's Disease. J. Neurosci. 2002, 22 (18), 7862-7872.

(43) Miller, J. P.; Holcomb, J.; Al-Ramahi, I.; de Haro, M.; Gafni, J.; Zhang, N.; Kim, E.; Sanhueza, M.; Torcassi, C.; Kwak, S.; Botas, J.; Hughes, R. E.; Ellerby, L. M. Matrix Metalloproteinases Are Modifiers of Huntingtin Proteolysis and Toxicity in Huntington's Disease. Neuron. 2010, 67 (2), 199-212.

(44) Yang, H.; Yang, S.; Jing, L.; Huang, L.; Chen, L.; Zhao, X.; Yang, W.; Pan, Y.; Yin, P.; Qin, Z. S.; Li, S.; Li, X.-J. Truncation of Mutant Huntingtin in Knock-in Mice Demonstrates Exon1 Huntingtin Is a Key Pathogenic Form. Nat. Commun. 2020, 11 (1), 2582.

(45) Mangiarini, L.; Sathasivam, K.; Seller, M.; Cozens, B.; Harper, A.; Hetherington, C.; Lawton, M.; Trottier, Y.; Lehrach, H.; Davies, S. W.; Bates, G. P. Exon 1 of the HD Gene with an Expanded CAG Repeat Is Sufficient to Cause a Progressive Neurological Phenotype in Transgenic Mice. Cell. 1996, 87 (3), 493-506.

(46) Nguyen, T.; Hamby, A.; Massa, S. M. Clioquinol Down-Regulates Mutant Huntingtin Expression in Vitro and Mitigates Pathology in a Huntington's Disease Mouse Model. PNAS. 2005, 102 (33), $11840-11845$.

(47) DiFiglia, M.; Sapp, E.; Chase, K.; Davies, S.; Bates, G. Aggregation of Huntingtin in Neuronal Intranculear Inclusions and Dystrophic Neurites in Brain. Science. 1997, 277 (5334), 1990-1993.

(48) Chen, S.; Berthelier, V.; Yang, W.; Wetzel, R. Polyglutamine Aggregation Behavior in Vitro Supports a Recruitment Mechanism of Cytotoxicity. J. Mol. Biol. 2001, 311 (1), 173-182.

(49) Chen, S.; Berthelier, V.; Hamilton, J. B.; O’Nuallai, B.; Wetzel, R. Amyloid-like Features of Polyglutamine Aggregates and Their Assembly Kinetics. Biochemistry. 2002, 41 (23), 73917399. 
(50) Poirier, M. A.; Li, H.; Macosko, J.; Cai, S.; Amzel, M.; Ross, C. A. Huntingtin Spheroids and Protofibrils as Precursors in Polyglutamine Fibrilization. J. Biol. Chem. 2002, 277 (43), 4103241037.

(51) Scherzinger, E.; Lurz, R.; Turmaine, M.; Mangiarini, L.; Hollenbach, B.; Hasenbank, R.; Bates, G. P.; Davies, S. W.; Lehrach, H.; Wanker, E. E. Huntingtin-Encoded Polyglutamine Expansions Form Amyloid-like Protein Aggregates In Vitro and In Vivo. Cell. 1997, 90 (3), 549-558.

(52) Scherzinger, E.; Sittler, A.; Schweiger, K.; Heiser, V.; Lurz, R.; Hasenbank, R.; Bates, G. P.; Lehrach, H.; Wanker, E. E. Self-Assembly of Polyglutamine-Containing Huntingtin Fragments into Amyloid-like Fibrils: Implications for Huntington's Disease Pathology. PNAS. 1999, 96 (8), 4604-4609.

(53) Wetzel, R. Physical Chemistry of Polyglutamine: Intriguing Tales of a Monotonous Sequence. $J$. Mol. Biol. 2012, 421 (4), 466-490.

(54) Legleiter, J.; Mitchell, E.; Lotz, G. P.; Sapp, E.; Ng, C.; DiFiglia, M.; Thompson, L. M.; Muchowski, P. J. Mutant Huntingtin Fragments Form Oligomers in a Polyglutamine LengthDependent Manner in Vitro and in Vivo. J. Biol. Chem. 2010, 285 (19), 14777-14790.

(55) Lunkes, A.; Mandel, J.-L. A Cellular Model That Recapitulates Major Pathogenic Steps of Huntington's Disease. Hum. Mol. Genet. 1998, 7 (9), 1355-1361.

(56) Schilling, G.; Becher, M. W.; Sharp, A. H.; Jinnah, H. A.; Duan, K.; Kotzuk, J. A.; Slunt, H. H.; Ratovitski, T.; Cooper, J. K.; Jenkins, N. A.; Copeland, N. G.; Price, D. L.; Ross, C. A.; Borchelt, D. R. Intranuclear Inclusions and Neuritic Aggregates in Transgenic Mice Expressing a Mutant NTerminal Fragment of Huntingtin. Hum. Mol. Genet. 1999, 8 (3), 397-407.

(57) Sahl, S. J.; Lau, L.; Vonk, W. I. M.; Weiss, L. E.; Frydman, J.; Moerner, W. E. Delayed Emergence of Subdiffraction-Sized Mutant Huntingtin Fibrils Following Inclusion Body Formation. Q. Rev. Biophys. 2016, 49.

(58) Lajoie, P.; Snapp, E. L. Formation and Toxicity of Soluble Polyglutamine Oligomers in Living Cells. Plos One. 2010, 5 (12).

(59) Lu, M.; Banetta, L.; Young, L. J.; Smith, E. J.; Bates, G. P.; Zaccone, A.; Schierle, G. S. K.; Tunnacliffe, A.; Kaminski, C. F. Live-Cell Super-Resolution Microscopy Reveals a Primary Role for Diffusion in Polyglutamine-Driven Aggresome Assembly. J. Biol. Chem. 2019, 294 (1), 257268.

(60) Kotler, S. A.; Tugarinov, V.; Schmidt, T.; Ceccon, A.; Libich, D. S.; Ghirlando, R.; Schwieters, C. D.; Clore, G. M. Probing Initial Transient Oligomerization Events Facilitating Huntingtin Fibril Nucleation at Atomic Resolution by Relaxation-Based NMR. PNAS. 2019, 116 (9), 3562-3571.

(61) Landrum, E.; Wetzel, R. Biophysical Underpinnings of the Repeat Length Dependence of Polyglutamine Amyloid Formation. J. Biol. Chem. 2014, 289 (15), 10254-10260.

(62) Olshina, M. A.; Angley, L. M.; Ramdzan, Y. M.; Tang, J.; Bailey, M. F.; Hill, A. F.; Hatters, D. M. Tracking Mutant Huntingtin Aggregation Kinetics in Cells Reveals Three Major Populations That Include an Invariant Oligomer Pool. J. Biol. Chem. 2010, 285 (28), 21807-21816.

(63) Walters, R. H.; Murphy, R. M. Aggregation Kinetics of Interrupted Polyglutamine Peptides. $J$. Mol. Biol. 2011, 412 (3), 505-519.

(64) Thakur, A. K.; Jayaraman, M.; Mishra, R.; Thakur, M.; Chellgren, V. M.; Byeon, I.-J. L.; Anjum, D. H.; Kodali, R.; Creamer, T. P.; Conway, J. F.; Gronenborn, A. M.; Wetzel, R. Polyglutamine Disruption of the Huntingtin Exon $1 \mathrm{~N}$ Terminus Triggers a Complex Aggregation Mechanism. Nat. Struct. Mol. Biol. 2009, 16 (4), 380-389.

(65) Arrasate, M.; Mitra, S.; Schweitzer, E. S.; Segal, M. R.; Finkbeiner, S. Inclusion Body Formation Reduces Levels of Mutant Huntingtin and the Risk of Neuronal Death. Nature. 2004, 431 (7010), 805-810.

(66) Gutekunst, C.-A.; Li, S.-H.; Yi, H.; Mulroy, J. S.; Kuemmerle, S.; Jones, R.; Rye, D.; Ferrante, R. J.; Hersch, S. M.; Li, X.-J. Nuclear and Neuropil Aggregates in Huntington's Disease: Relationship to Neuropathology. J. Neurosci. 1999, 19 (7), 2522-2534. 
Sahl, S. J.; Weiss, L. E.; Duim, W. C.; Frydman, J.; Moerner, W. E. Cellular Inclusion Bodies of Mutant Huntingtin Exon 1 Obscure Small Fibrillar Aggregate Species. Sci. Rep. 2012, 2 (1), 1-7.

(68) Duim, W. C.; Chen, B.; Frydman, J.; Moerner, W. E. Sub-Diffraction Imaging of Huntingtin Protein Aggregates by Fluorescence Blink-Microscopy and Atomic Force Microscopy. Chem. Phys. Chem 2011, 12 (13), 2387-2390.

(69) Bäuerlein, F. J. B.; Saha, I.; Mishra, A.; Kalemanov, M.; Martínez-Sánchez, A.; Klein, R.; Dudanova, I.; Hipp, M. S.; Hartl, F. U.; Baumeister, W.; Fernández-Busnadiego, R. In Situ Architecture and Cellular Interactions of PolyQ Inclusions. Cell. 2017, 171 (1), 179-187.e10.

(70) Pieri, L.; Madiona, K.; Bousset, L.; Melki, R. Fibrillar $\alpha$-Synuclein and Huntingtin Exon 1 Assemblies Are Toxic to the Cells. Biophys. J. 2012, 102 (12), 2894-2905.

(71) Nekooki-Machida, Y.; Kurosawa, M.; Nukina, N.; Ito, K.; Oda, T.; Tanaka, M. Distinct Conformations of in Vitro and in Vivo Amyloids of Huntingtin-Exon1 Show Different Cytotoxicity. PNAS. 2009, 106 (24), 9679-9684.

(72) Yang, W.; Dunlap, J. R.; Andrews, R. B.; Wetzel, R. Aggregated Polyglutamine Peptides Delivered to Nuclei Are Toxic to Mammalian Cells. Hum. Mol. Genet. 2002, 11 (23), 2905-2917.

(73) Kar, K.; Arduini, I.; Drombosky, K. W.; van der Wel, P. C. A.; Wetzel, R. D-Polyglutamine Amyloid Recruits 1-Polyglutamine Monomers and Kills Cells. J. Mol. Biol. 2014, 426 (4), 816829.

(74) Drombosky, K. W.; Rode, S.; Kodali, R.; Jacob, T. C.; Palladino, M. J.; Wetzel, R. Mutational Analysis Implicates the Amyloid Fibril as the Toxic Entity in Huntington's Disease. Neurobiol. Dis. 2018, 120, 126-138.

(75) Takahashi, Y.; Okamoto, Y.; Popiel, H. A.; Fujikake, N.; Toda, T.; Kinjo, M.; Nagai, Y. Detection of Polyglutamine Protein Oligomers in Cells by Fluorescence Correlation Spectroscopy. J. Biol. Chem. 2007, 282 (33), 24039-24048.

(76) Sathasivam, K.; Lane, A.; Legleiter, J.; Warley, A.; Woodman, B.; Finkbeiner, S.; Paganetti, P.; Muchowski, P. J.; Wilson, S.; Bates, G. P. Identical Oligomeric and Fibrillar Structures Captured from the Brains of R6/2 and Knock-in Mouse Models of Huntington's Disease. Hum. Mol. Genet.2009, 19 (1), 65-78.

(77) Marcellin, D.; Abramowski, D.; Young, D.; Richter, J.; Weiss, A.; Marcel, A.; Maassen, J.; Kauffmann, M.; Bibel, M.; Shimshek, D. R.; Faull, R. L. M.; Bates, G. P.; Kuhn, R. R.; Putten, P. H. V. der; Schmid, P.; Lotz, G. P. Fragments of HdhQ150 Mutant Huntingtin Form a Soluble Oligomer Pool That Declines with Aggregate Deposition upon Aging. PLOS ONE. 2012, 7 (9), e44457.

(78) Ossato, G.; Digman, M. A.; Aiken, C.; Lukacsovich, T.; Marsh, J. L.; Gratton, E. A Two-Step Path to Inclusion Formation of Huntingtin Peptides Revealed by Number and Brightness Analysis. Biophys. J. 2010, 98 (12), 3078-3085.

(79) Huang, B.; Lucas, T.; Kueppers, C.; Dong, X.; Krause, M.; Bepperling, A.; Buchner, J.; Voshol, H.; Weiss, A.; Gerrits, B.; Kochanek, S. Scalable Production in Human Cells and Biochemical Characterization of Full-Length Normal and Mutant Huntingtin. PLoS ONE. 2015, 10 (3), e0121055.

(80) Sahoo, B.; Arduini, I.; Drombosky, K. W.; Kodali, R.; Sanders, L. H.; Greenamyre, J. T.; Wetzel, R. Folding Landscape of Mutant Huntingtin Exon1: Diffusible Multimers, Oligomers and Fibrils, and No Detectable Monomer. PLoS ONE. 2016, 11 (6), 1-22.

(81) Posey, A. E.; Ruff, K. M.; Harmon, T. S.; Crick, S. L.; Li, A.; Diamond, M. I.; Pappu, R. V. Profilin Reduces Aggregation and Phase Separation of Huntingtin N-Terminal Fragments by Preferentially Binding to Soluble Monomers and Oligomers. J. Biol. Chem. 2018, 293 (10), 3734-3746.

(82) Leitman, J.; Ulrich Hartl, F.; Lederkremer, G. Z. Soluble Forms of PolyQ-Expanded Huntingtin Rather than Large Aggregates Cause Endoplasmic Reticulum Stress. Nat. Commun. 2013, 4 (1), 2753.

(83) Jiang, Y.; Chadwick, S. R.; Lajoie, P. Endoplasmic Reticulum Stress: The Cause and Solution to Huntington's Disease? Brain. Research. 2016, 1648, 650-657. 
Ueda, M.; Li, S.; Itoh, M.; Wang, M.; Hayakawa, M.; Islam, S.; Tana; Nakagawa, K.; Chen, H.; Nakagawa, T. Expanded Polyglutamine Embedded in the Endoplasmic Reticulum Causes Membrane Distortion and Coincides with Bax Insertion. Biochem. Biophys. Res. Commun. 2016, 474 (2), 259-263.

(85) Ramdzan, Y. M.; Trubetskov, M. M.; Ormsby, A. R.; Newcombe, E. A.; Sui, X.; Tobin, M. J.; Bongiovanni, M. N.; Gras, S. L.; Dewson, G.; Miller, J. M. L.; Finkbeiner, S.; Moily, N. S.; Niclis, J.; Parish, C. L.; Purcell, A. W.; Baker, M. J.; Wilce, J. A.; Waris, S.; Stojanovski, D.; Böcking, T.; Ang, C.-S.; Ascher, D. B.; Reid, G. E.; Hatters, D. M. Huntingtin Inclusions Trigger Cellular Quiescence, Deactivate Apoptosis, and Lead to Delayed Necrosis. Call. Rep. 2017, 19 (5), 919 927.

(86) Crick, S. L.; Ruff, K. M.; Garai, K.; Frieden, C.; Pappu, R. V. Unmasking the Roles of N- and CTerminal Flanking Sequences from Exon 1 of Huntingtin as Modulators of Polyglutamine Aggregation. PNAS. 2013, 110 (50), 20075-20080.

(87) Sivanandam, V. N.; Jayaraman, M.; Hoop, C. L.; Kodali, R.; Wetzel, R.; van der Wel, P. C. A. The Aggregation-Enhancing Huntingtin N-Terminus Is Helical in Amyloid Fibrils. J. Am. Chem. Soc. 2011, 133 (12), 4558-4566.

(88) Burke, K. A.; Kauffman, K. J.; Umbaugh, C. S.; Frey, S. L.; Legleiter, J. The Interaction of Polyglutamine Peptides with Lipid Membranes Is Regulated by Flanking Sequences Associated with Huntingtin. J. Biol. Chem. 2013, 288 (21), 14993-15005.

(89) Mishra, R.; Jayaraman, M.; Roland, B. P.; Landrum, E.; Fullam, T.; Kodali, R.; Thakur, A. K.; Arduini, I.; Wetzel, R. Inhibiting the Nucleation of Amyloid Structure in a Huntingtin Fragment by Targeting $\alpha$-Helix-Rich Oligomeric Intermediates. J. Mol. Biol. 2012, 415 (5), 900-917.

(90) Jayaraman, M.; Kodali, R.; Sahoo, B.; Thakur, A. K.; Mayasundari, A.; Mishra, R.; Peterson, C. B.; Wetzel, R. Slow Amyloid Nucleation via $\alpha$-Helix-Rich Oligomeric Intermediates in Short Polyglutamine-Containing Huntingtin Fragments. J. Mol. Biol. 2012, 415 (5), 881-899.

(91) Michalek, M.; Salnikov, E. S.; Werten, S.; Bechinger, B. Membrane Interactions of the Amphipathic Amino Terminus of Huntingtin. Biochemistry. 2013, 52 (5), 847-858.

(96) Isas, J. M.; Langen, R.; Siemer, A. B. Solid-State Nuclear Magnetic Resonance on the Static and Dynamic Domains of Huntingtin Exon-1 Fibrils. Biochemistry. 2015, 54 (25), 3942-3949.

(97) Tam, S.; Spiess, C.; Auyeung, W.; Joachimiak, L.; Chen, B.; Poirier, M. A.; Frydman, J. The Chaperonin TRiC Blocks a Huntingtin Sequence Element That Promotes the Conformational Switch to Aggregation. Nat. Struct. Mol. Biol. 2009, 16 (12), 1279-1285.

(98) Hoop, C. L.; Lin, H.-K.; Kar, K.; Hou, Z.; Poirier, M. A.; Wetzel, R.; van der Wel, P. C. A. Polyglutamine Amyloid Core Boundaries and Flanking Domain Dynamics in Huntingtin Fragment Fibrils Determined by Solid-State Nuclear Magnetic Resonance. Biochemistry. 2014, 53 (42), 6653-6666.

(99) Bugg, C. W.; Isas, J. M.; Fischer, T.; Patterson, P. H.; Langen, R. Structural Features and Domain Organization of Huntingtin Fibrils. J. Biol. Chem. 2012, 287 (38), 31739-31746.

(100) Colby, D. W.; Garg, P.; Holden, T.; Chao, G.; Webster, J. M.; Messer, A.; Ingram, V. M.; Wittrup, K. D. Development of a Human Light Chain Variable Domain (VL) Intracellular Antibody 
Specific for the Amino Terminus of Huntingtin via Yeast Surface Display. J. Mol. Biol. 2004, 342 (3), 901-912.

(101) Monsellier, E.; Redeker, V.; Ruiz-Arlandis, G.; Bousset, L.; Melki, R. Molecular Interaction between the Chaperone Hsc70 and the N-Terminal Flank of Huntingtin Exon 1 Modulates Aggregation. J. Biol. Chem. 2015, 290 (5), 2560-2576.

(102) Gutekunst, C. A.; Levey, A. I.; Heilman, C. J.; Whaley, W. L.; Yi, H.; Nash, N. R.; Rees, H. D.; Madden, J. J.; Hersch, S. M. Identification and Localization of Huntingtin in Brain and Human Lymphoblastoid Cell Lines with Anti-Fusion Protein Antibodies. PNAS. 1995, 92 (19), 87108714.

(103) Gutekunst, C.-A.; Li, S.-H.; Yi, H.; Ferrante, R. J.; Li, X.-J.; Hersch, S. M. The Cellular and Subcellular Localization of Huntingtin-Associated Protein 1 (HAP1): Comparison with Huntingtin in Rat and Human. J. Neurosci. 1998, 18 (19), 7674-7686.

(104) Kegel, K. B.; Kim, M.; Sapp, E.; McIntyre, C.; Castaño, J. G.; Aronin, N.; DiFiglia, M. Huntingtin Expression Stimulates Endosomal-Lysosomal Activity, Endosome Tubulation, and Autophagy. $J$. Neurosci. 2000, 20 (19), 7268-7278.

(105) Qin, Z.-H.; Wang, Y.; Sapp, E.; Cuiffo, B.; Wanker, E.; Hayden, M. R.; Kegel, K. B.; Aronin, N.; DiFiglia, M. Huntingtin Bodies Sequester Vesicle-Associated Proteins by a PolyprolineDependent Interaction. J. Neurosci. 2004, 24 (1), 269-281.

(106) Suopanki, J.; Götz, C.; Lutsch, G.; Schiller, J.; Harjes, P.; Herrmann, A.; Wanker, E. E. Interaction of Huntingtin Fragments with Brain Membranes - Clues to Early Dysfunction in Huntington's Disease. J. Neurochem. 2006, 96 (3), 870-884.

(107) Valencia, A.; Reeves, P. B.; Sapp, E.; Li, X.; Alexander, J.; Kegel, K. B.; Chase, K.; Aronin, N.; DiFiglia, M. Mutant Huntingtin and Glycogen Synthase Kinase 3- $\beta$ Accumulate in Neuronal Lipid Rafts of a Presymptomatic Knock-in Mouse Model of Huntington's Disease. J. Neurosci. Res. 2010, 88 (1), 179-190.

(108) Shirendeb, U.; Reddy, A. P.; Manczak, M.; Calkins, M. J.; Mao, P.; Tagle, D. A.; Hemachandra Reddy, P. Abnormal Mitochondrial Dynamics, Mitochondrial Loss and Mutant Huntingtin Oligomers in Huntington's Disease: Implications for Selective Neuronal Damage. Hum. Mol. Genet. 2011, 20 (7), 1438-1455.

(109) Burke, K. A.; Yates, E. A.; Legleiter, J. Biophysical Insights into How Surfaces, Including Lipid Membranes, Modulate Protein Aggregation Related to Neurodegeneration. Front. Neurol. 2013, 4.

(110) Gorbenko, G. P.; Kinnunen, P. K. J. The Role of Lipid-Protein Interactions in Amyloid-Type Protein Fibril Formation. Chem.Phys. Lipid. 2006, 141 (1), 72-82.

(111) Burke, K. A.; Hensal, K. M.; Umbaugh, C. S.; Chaibva, M.; Legleiter, J. Huntingtin Disrupts Lipid Bilayers in a PolyQ-Length Dependent Manner. Biochim. Biophys. Acta. Biomembr. 2013, 1828 (8), 1953-1961.

(112) Burke, K. A.; Yates, E. A.; Legleiter, J. Amyloid-Forming Proteins Alter the Local Mechanical Properties of Lipid Membranes. Biochemistry. 2013, 52 (5), 808-817.

(113) Monsellier, E.; Bousset, L.; Melki, R. $\alpha$-Synuclein and Huntingtin Exon 1 Amyloid Fibrils Bind Laterally to the Cellular Membrane. Sci. Rep. 2016, 6 (1), 19180.

(114) Gao, X.; Campbell, W. A.; Chaibva, M.; Jain, P.; Leslie, A. E.; Frey, S. L.; Legleiter, J. Cholesterol Modifies Huntingtin Binding to, Disruption of, and Aggregation on Lipid Membranes. Biochemistry. 2016, 55 (1), 92-102.

(115) Chaibva, M.; Gao, X.; Jain, P.; Campbell, W. A.; Frey, S. L.; Legleiter, J. Sphingomyelin and GM1 Influence Huntingtin Binding to, Disruption of, and Aggregation on Lipid Membranes. ACS. Omega. 2018, 3 (1), 273-285.

(116) Michalek, M.; Salnikov, E. S.; Bechinger, B. Structure and Topology of the Huntingtin 1-17 Membrane Anchor by a Combined Solution and Solid-State Nmr Approach. Biophys. J. 2013, 105 (3), 699-710.

(117) Rockabrand, E.; Slepko, N.; Pantalone, A.; Nukala, V. N.; Kazantsev, A.; Marsh, J. L.; Sullivan, P. G.; Steffan, J. S.; Sensi, S. L.; Thompson, L. M. The First 17 Amino Acids of Huntingtin 
Modulate Its Sub-Cellular Localization, Aggregation and Effects on Calcium Homeostasis. Hum. Mol. Genet.2007, 16 (1), 61-77.

(118) Nagarajan, A.; Jawahery, S.; Matysiak, S. The Effects of Flanking Sequences in the Interaction of Polyglutamine Peptides with a Membrane Bilayer. J. Phys. Chem. B. 2014, 118 (24), 6368-6379.

(119) Côté, S.; Wei, G.; Mousseau, N. Atomistic Mechanisms of Huntingtin N-Terminal Fragment Insertion on a Phospholipid Bilayer Revealed by Molecular Dynamics Simulations. Proteins. 2014, 82 (7), 1409-1427.

(120) Karanji, A. K.; Beasley, M.; Sharif, D.; Ranjbaran, A.; Legleiter, J.; Valentine, S. J. Investigating the Interactions of the First 17 Amino Acid Residues of Huntingtin with Lipid Vesicles Using Mass Spectrometry and Molecular Dynamics. J. Mass. Spectrom. 2020, 55 (1), e4470.

(121) Morshedi, D.; Ebrahim-Habibi, A.; Moosavi-Movahedi, A. A.; Nemat-Gorgani, M. Chemical Modification of Lysine Residues in Lysozyme May Dramatically Influence Its Amyloid Fibrillation. Biochim. Biophys. Acta. 2010, 1804 (4), 714-722.

(122) Davern, S.; Murphy, C. L.; O’Neill, H.; Wall, J. S.; Weiss, D. T.; Solomon, A. Effect of Lysine Modification on the Stability and Cellular Binding of Human Amyloidogenic Light Chains. Biochim. Biophys. Acta .2011, 1812 (1), 32-40.

(123) Groveman, B. R.; Kraus, A.; Raymond, L. D.; Dolan, M. A.; Anson, K. J.; Dorward, D. W.; Caughey, B. Charge Neutralization of the Central Lysine Cluster in Prion Protein (PrP) Promotes PrP(Sc)-like Folding of Recombinant PrP Amyloids. J. Biol. Chem. 2015, 290 (2), 1119-1128.

(124) Sinha, S.; Lopes, D. H. J.; Bitan, G. A Key Role for Lysine Residues in Amyloid $\beta$-Protein Folding, Assembly, and Toxicity. ACS. Chem. Neurosci. 2012, 3 (6), 473-481.

(125) Li, W.; Sperry, J. B.; Crowe, A.; Trojanowski, J. Q.; Smith, A. B.; Lee, V. M.-Y. Inhibition of Tau Fibrillization by Oleocanthal via Reaction with the Amino Groups of Tau. J. Neurochem. 2009, 110 (4), 1339-1351.

(126) Chaibva, M.; Jawahery, S.; Pilkington, A. W.; Arndt, J. R.; Sarver, O.; Valentine, S.; Matysiak, S.; Legleiter, J. Acetylation within the First 17 Residues of Huntingtin Exon 1 Alters Aggregation and Lipid Binding. Biophys. J. 2016, 111 (2), 349-362.

(127) Arndt, J. R.; Brown, R. J.; Burke, K. A.; Legleiter, J.; Valentine, S. J. Lysine Residues in the NTerminal Huntingtin Amphipathic $\alpha$-Helix Play a Key Role in Peptide Aggregation. J. Mass. Spectrom. 2015, 50 (1), 117-126.

(128) Côté, S.; Binette, V.; Salnikov, E. S.; Bechinger, B.; Mousseau, N. Probing the Huntingtin 1-17 Membrane Anchor on a Phospholipid Bilayer by Using All-Atom Simulations. Biophys. J. 2015, 108 (5), 1187-1198.

(129) Tao, M.; Pandey, N. K.; Barnes, R.; Han, S.; Langen, R. Structure of Membrane-Bound Huntingtin Exon 1 Reveals Membrane Interaction and Aggregation Mechanisms. Structure. 2019, 27 (10), 1570-1580. e4.

(130) Pennuto, M.; Palazzolo, I.; Poletti, A. Post-Translational Modifications of Expanded Polyglutamine Proteins: Impact on Neurotoxicity. Hum. Mol. Genet. 2009, 18 (R1), R40-R47.

(131) Aiken, C. T.; Steffan, J. S.; Guerrero, C. M.; Khashwji, H.; Lukacsovich, T.; Simmons, D.; Purcell, J. M.; Menhaji, K.; Zhu, Y.-Z.; Green, K.; LaFerla, F.; Huang, L.; Thompson, L. M.; Marsh, J. L. Phosphorylation of Threonine 3 IMPLICATIONS FOR HUNTINGTIN AGGREGATION AND NEUROTOXICITY. J. Biol. Chem. 2009, 284 (43), 29427-29436.

(132) Steffan, J. S.; Agrawal, N.; Pallos, J.; Rockabrand, E.; Trotman, L. C.; Slepko, N.; Illes, K.; Lukacsovich, T.; Zhu, Y.-Z.; Cattaneo, E.; Pandolfi, P. P.; Thompson, L. M.; Marsh, J. L. SUMO Modification of Huntingtin and Huntington's Disease Pathology. Science. 2004, 304 (5667), 100104.

(133) Ehrnhoefer, D. E.; Sutton, L.; Hayden, M. R. Small Changes, Big Impact: Posttranslational Modifications and Function of Huntingtin in Huntington Disease. Neuroscientist. 2011, 17 (5), 475-492.

(134) Díaz-Hernández, M.; Valera, A. G.; Morán, M. A.; Gómez-Ramos, P.; Alvarez-Castelao, B.; Castaño, J. G.; Hernández, F.; Lucas, J. J. Inhibition of 26S Proteasome Activity by Huntingtin 
Filaments but Not Inclusion Bodies Isolated from Mouse and Human Brain. J. Neurochem. 2006, 98 (5), 1585-1596.

(135) Sap, K. A.; Guler, A. T.; Bezstarosti, K.; Bury, A. E.; Juenemann, K.; Demmers, J. A. A.; Reits, E. A. Global Proteome and Ubiquitinome Changes in the Soluble and Insoluble Fractions of Q175 Huntington Mice Brains. Mol. Cell. Proteomics. 2019, 18 (9), 1705-1720.

(136) Watkin, E. E.; Arbez, N.; Waldron-Roby, E.; O’Meally, R.; Ratovitski, T.; Cole, R. N.; Ross, C. A. Phosphorylation of Mutant Huntingtin at Serine 116 Modulates Neuronal Toxicity. PLoS ONE. 2014, 9 (2), e88284.

(137) Thompson, L. M.; Aiken, C. T.; Kaltenbach, L. S.; Agrawal, N.; Illes, K.; Khoshnan, A.; MartinezVincente, M.; Arrasate, M.; O’Rourke, J. G.; Khashwji, H.; Lukacsovich, T.; Zhu, Y.-Z.; Lau, A. L.; Massey, A.; Hayden, M. R.; Zeitlin, S. O.; Finkbeiner, S.; Green, K. N.; LaFerla, F. M.; Bates, G.; Huang, L.; Patterson, P. H.; Lo, D. C.; Cuervo, A. M.; Marsh, J. L.; Steffan, J. S. IKK Phosphorylates Huntingtin and Targets It for Degradation by the Proteasome and Lysosome. $J$. Cell. Biol. 2009, 187 (7), 1083-1099.

(138) Gu, X.; Greiner, E. R.; Mishra, R.; Kodali, R.; Osmand, A.; Finkbeiner, S.; Steffan, J. S.; Thompson, L. M.; Wetzel, R.; Yang, X. W. Serines 13 and 16 Are Critical Determinants of FullLength Human Mutant Huntingtin Induced Disease Pathogenesis in HD Mice. Neuron. 2009, 64 (6), 828-840.

(139) Humbert, S.; Saudou, F. Huntingtin Phosphorylation and Signaling Pathways That Regulate Toxicity in Huntington's Disease. Clin. Neurosci. Res. 2003, 3 (3), 149-155.

(140) Pardo, A. D.; Maglione, V.; Alpaugh, M.; Horkey, M.; Atwal, R. S.; Sassone, J.; Ciammola, A.; Steffan, J. S.; Fouad, K.; Truant, R.; Sipione, S. Ganglioside GM1 Induces Phosphorylation of Mutant Huntingtin and Restores Normal Motor Behavior in Huntington Disease Mice. PNAS. 2012, 109 (9), 3528-3533.

(141) Elbaum, M. B.; Zondlo, N. J. OGlcNAcylation and Phosphorylation Have Similar Structural Effects in $\alpha$-Helices: Post-Translational Modifications as Inducible Start and Stop Signals in $\alpha-$ Helices, with Greater Structural Effects on Threonine Modification. Biochemistry. 2014, 53 (14), 2242-2260.

(142) Mishra, R.; Hoop, C. L.; Kodali, R.; Sahoo, B.; van der Wel, P. C. A.; Wetzel, R. Serine Phosphorylation Suppresses Huntingtin Amyloid Accumulation by Altering Protein Aggregation Properties. J. Mol. Biol. 2012, 424 (1), 1-14.

(143) Cong, X.; Held, J. M.; DeGiacomo, F.; Bonner, A.; Chen, J. M.; Schilling, B.; Czerwieniec, G. A.; Gibson, B. W.; Ellerby, L. M. Mass Spectrometric Identification of Novel Lysine Acetylation Sites in Huntingtin. Mol. Cell. Proteomics. 2011, 10 (10), M111.009829-M111.009829.

(144) Chiki, A.; DeGuire, S. M.; Ruggeri, F. S.; Sanfelice, D.; Ansaloni, A.; Wang, Z.-M.; Cendrowska, U.; Burai, R.; Vieweg, S.; Pastore, A.; Dietler, G.; Lashuel, H. A. Mutant Exon1 Huntingtin Aggregation Is Regulated by T3 Phosphorylation-Induced Structural Changes and Crosstalk between T3 Phosphorylation and Acetylation at K6. Angew. Chem. Int. Ed. 2017, 56 (19), 52025207.

(145) Subramaniam, S.; Sixt, K. M.; Barrow, R.; Snyder, S. H. Rhes, a Striatal Specific Protein, Mediates Mutant-Huntingtin Cytotoxicity. Science. 2009, 324 (5932), 1327-1330.

(146) O'Rourke, J. G.; Gareau, J. R.; Ochaba, J.; Song, W.; Raskó, T.; Reverter, D.; Lee, J.; Monteys, A. M.; Pallos, J.; Mee, L.; Vashishtha, M.; Apostol, B. L.; Nicholson, T. P.; Illes, K.; Zhu, Y.-Z.; Dasso, M.; Bates, G. P.; Difiglia, M.; Davidson, B.; Wanker, E. E.; Marsh, J. L.; Lima, C. D.; Steffan, J. S.; Thompson, L. M. SUMO-2 and PIAS1 Modulate Insoluble Mutant Huntingtin Protein Accumulation. Cell. Rep. 2013, 4 (2), 362-375.

(147) Ochaba, J.; Monteys, A. M.; O’Rourke, J. G.; Reidling, J. C.; Steffan, J. S.; Davidson, B. L.; Thompson, L. M. PIAS1 Regulates Mutant Huntingtin Accumulation and Huntington's DiseaseAssociated Phenotypes in Vivo. Neuron. 2016, 90 (3), 507-520. 


\section{SUMOylation Prevents Huntingtin Fibrillization and Localization onto Lipid Membranes ${ }^{1}$}

\subsection{Abstract}

Huntington's disease (HD), a genetic neurodegenerative disease, is caused by an expanded polyglutamine (polyQ) domain in the first exon of the huntingtin protein (htt). PolyQ expansion destabilizes protein structure, resulting in aggregation into a variety of oligomers, protofibrils, and fibrils. Beyond the polyQ domain, adjacent protein sequences influence the aggregation process. Specifically, the first $17 \mathrm{~N}$-terminal amino acids (Nt17) directly preceding the polyQ domain promote the formation of $\alpha$-helix-rich oligomers that represent intermediate species associated with fibrillization. Due to its propensity to form an amphipathic $\alpha$-helix, Nt17 also facilitates lipid binding. Three lysine residues (K6, K9, and K15) within Nt17 can be SUMOylated, which modifies htt's accumulation and toxicity within cells in a variety of HD models. The impact of SUMOylation on htt aggregation and direct interaction with lipid membranes was investigated. SUMOylation of htt-exon1 inhibited fibril formation while promoting larger, amorphous aggregate species. These amorphous aggregates were SDS soluble but nonetheless exhibited levels of $\beta$-sheet structure similar to that of htt-exon1 fibrils. In addition, SUMOylation prevented htt binding, aggregation, and accumulation on model lipid bilayers comprised of total brain lipid extract. Collectively, these observations demonstrate that SUMOylation promotes a distinct htt aggregation pathway that may affect htt toxicity.

\footnotetext{
${ }^{1}$ Reprinted with permission from Faezeh Sedighi, Adewale Adegbuyiro, and Justin Legleiter ACS. Chem. Neurosci. 202011 (3), 328-343 DOI: 10.1021/acschemneuro.9b00509. Copyright 2020 American Chemical Society.
} 


\subsection{Introduction}

Huntington disease (HD) is an autosomal dominant neurodegenerative disorder caused by expansion of a polyglutamine (polyQ) stretch beyond a critical threshold in the huntingtin (htt) protein. ${ }^{1} 1$ Once the critical threshold is reached, there is a strong correlation between polyQ length and disease pathology, i.e. age of onset. ${ }^{2,3}$ In addition, expanded polyQ induces aberrant htt aggregation into fibrils similar to amyloids associated with other neurodegenerative diseases. ${ }^{4-7}$ The rate of fibril formation also accelerates with increasing polyQ length. ${ }^{4,5,8} \mathrm{Htt}$ aggregation leads to the eventual formation of large, proteinaceous inclusion bodies within the cytoplasm and nucleus. ${ }^{9}$ These inclusions can damage ER and nuclear membranes. ${ }^{10,11} \mathrm{Htt}$ aggregation is a complex, heterogeneous process with a variety of different aggregate species forming on and off pathway to fibril formation. These smaller, intermediate aggregates include a variety of oligomers and protofibrils. ${ }^{8,12-18}$ Cell survival analysis suggests that, rather than inclusions, these nanoscale aggregates that are diffusely distributed throughout the cell represent the most toxic species of mutant htt. ${ }^{19-22}$ Importantly, this diffuse milieu of htt within cells can contain a mixture of oligomers and fibrils, ${ }^{12,23-25}$ making it difficult to precisely assign toxic mechanisms to specific htt aggregate species.

While the expanded polyQ domain in the first exon of htt is responsible for aggregation, flanking sequences surrounding the polyQ significantly influence the aggregation process. A polyproline (polyP) region directly adjacent to the C-terminal side of the polyQ domain stabilizes an aggregation incompetent monomeric conformation that retards fibril formation. ${ }^{26,27}$ Unlike polyP, the first 17 amino acids of the N-terminus of htt (Nt17) that directly precedes the polyQ domain enhance fibrillization. ${ }^{14,27}$ In solution $\mathrm{Nt} 17$ predominately behaves like an intrinsically disordered sequence, but it adopts an amphipathic $\alpha$-helical conformation upon interaction with a binding partner, which underlies its affinity for lipid membranes. ${ }^{28,29}$ This ability to form an 
amphipathic ahelix is implicated in the nucleation of htt aggregation as Nt17 domains selfassociate to form $\alpha$-helix rich oligomers, facilitating fibrillization by bringing polyQ domains into close proximity. ${ }^{14,27,30}$ As oligomers ${ }^{31,32}$ and fibrils ${ }^{33,34}$ have been demonstrated to be toxic, Nt17 mediated oligomerization associated with fibril nucleation may play a crucial role in HD pathogenesis. ${ }^{35}$ Indeed, Nt17 appears to be a viable target for inhibiting htt aggregation. ${ }^{30,36,37} \mathrm{Htt}$ is a multifunctional protein, ${ }^{38}$ and many of these functions are associated with its ability to interact directly with a variety of lipid surfaces. ${ }^{39,40}$ For example, htt localizes to brain membrane fractions, ${ }^{41}$ including the ER, ${ }^{22,42-45}$ mitochondria, ${ }^{46-51}$ and plasma membrane. ${ }^{52}$ Additionally, htt participates in the transport of lipid vesicles (endocytic, synaptic, or lysosomal). ${ }^{53-55}$ The interaction of htt-exon1 with lipid membranes is facilitated by $\mathrm{Nt17,29,56,57}$ and htt with expanded polyQ domains disrupts a variety of subcellular membranes ${ }^{58,59}$ While it is clear that lipids alter htt aggregation, literature reports have suggested both enhanced ${ }^{50,60}$ and inhibited ${ }^{29,36,61-64} \mathrm{htt}$ fibrilization in the presence of membranes, depending on the lipid system used.

The propensity of Nt17 to bind membranes or self-associate can be regulated by several posttranslational modifications (PTMs), including phosphorylation, ${ }^{65-68}$ acetylation, ${ }^{69}$ oxidation, ${ }^{70}$ ubiquitination, ${ }^{9,71}$ and SUMOylation. ${ }^{72,73}$ These PTMs in Nt17 have a pronounced impact on htt aggregation and fibril stability. For example, phosphorylation of serine 13 and 16 (S13 and S16) reduces pathogenesis and accumulation of htt in transgenic mouse models. ${ }^{68}$ Phosphomimetic mutations at S13 and S16 in a variety of htt systems inhibit fibrillization and aggregate stability. ${ }^{74}$ The inhibition of fibrillization by phosphorylation appears related to alterations in the nucleation process associated with the formation of the $\alpha$-helical-rich oligomers facilitated by Nt17. ${ }^{74}$ In addition, phosphorylation at threonine 3 (T3) occurs within cells and tissues relevant to HD, and phosphorylation of $\mathrm{T} 3$ enhanced htt aggregation in drosophila models of HD with reduced 
toxicity. ${ }^{65}$ Three lysine residues (K6, K9, and K15) in Nt17 can undergo acetylation, ubiquitination, or SUMOylation. With lysine residues playing a potentially important role in the formation of $\alpha$-helical oligomers, ${ }^{75}$ PTMs of lysine could readily impact htt aggregation. Of the three lysine residues, $\mathrm{K} 9$ is the most readily acetylated in $\mathrm{Nt} 17$, both in vivo ${ }^{69}$ and in vitro. ${ }^{36}$ When chemically acetylating lysine residues in htt (resulting in a mixture of $\mathrm{K} 6, \mathrm{~K} 9$, and $\mathrm{K} 15$ being acetylated), fibrillization was inhibited. ${ }^{36}$ However, the aggregation of synthetic htt peptides was unaffected when individual lysine residues were acetylated. ${ }^{76}$

While the above-discussed PTMs alter the local physicochemical properties of discrete residues in htt, ubiquitination and SUMOylation of htt results in the covalent attachment of relatively large proteins directly to $\mathrm{Nt} 17$ via lysine residues. ${ }^{72,77}$ Ubiquitination is known to mark proteins for degradation, $9,71,78$ alter their cellular location, modify protein activity, and promote/inhibit protein interactions. ${ }^{79}$ The small ubiquitin-like modifier (SUMO) proteins are remarkably similar to ubiquitin in structure and share similarities in their enzymatic machinery mediating their conjugation to proteins. ${ }^{80}$ In htt-exon1, K6 and K9 are preferentially SUMOylated; although, K15 can be SUMOylated to a lesser extent. ${ }^{72}$ SUMOylation competes with ubiquitination for the same lysine residues in Nt17; however, in terms of toxicity, these two PTMs display opposing effects when introduced into Drosophila models of HD. ${ }^{72}$ While ubiquitination tags htt for degradation resulting in reduced toxicity, ${ }^{81,82}$ SUMOylation impairs Previous studies demonstrate that htt SUMOylation impacts aggregation and toxicity in a cellular environment; ${ }^{72,73,83,84}$ however, biophysical details of the impact of SUMO on htt aggregation remain unclear. Furthermore, it is plausible that SUMOylation of htt via Nt17 would alter the ability of htt to interact with lipid membranes. Here, we investigate the direct impact of SUMOylation on the aggregate formation and morphology of a mutant htt-exon 1 protein in the 
presence and absence of lipid membranes. We demonstrate that SUMOylation prevents fibrillization and promotes the formation of large amorphous htt aggregates that are SDS soluble. In addition, SUMOylation abolishes htt's ability to bind and disrupt lipid membranes. Collectively, our observations shed light on the physicochemical properties of diffuse htt aggregates promoted by SUMOylation.

\subsection{Materials and Methods}

\subsubsection{Expression and purification of GST-htt-exon1 fusion protein}

A glutathione S-transferase (GST)-htt-exon1 fusion protein containing 46 repeats of glutamine residues was expressed and purified as previously described. ${ }^{85}$ In summary, GST-httexon1(46Q) expression was induced in Escherichia coli with $1 \mathrm{mM}$ isopropyle $\beta$-d-thiogalactoside for $4 \mathrm{~h}$ at $30{ }^{\circ} \mathrm{C}$. The cells were lysed with lysosome $(0.5 \mathrm{mg} / \mathrm{mL})$, and the fusion protein was purified by liquid chromatography (LPLC, BioRad) using a GST affinity column. To determine protein purity and verify the relevant fractions, gel electrophoresis was performed. Cleavage of the GST moiety by the protease factor Xa releases htt-exon1(46Q), initiating aggregation. Fresh, unfrozen GST-htt-exon1(46Q) fusion protein was used for all experiments. To remove potentially pre-existing aggregates prior to cleavage of GST, stock solutions of the fusion proteins were centrifuged at $20,000 \mathrm{~g}$ for $30 \mathrm{~min}$ at $4{ }^{\circ} \mathrm{C}$. All experiments were carried out in an aggregation buffer (50 mM Tris-HCl, 150 mM NaCl, pH 7.5).

\subsubsection{In Vitro htt-exon1 SUMOylation}

SUMO1 modification assay was performed with a SUMOylation kit (Active Motif) with slight modifications to the protocol described in the manual. To begin to remove the GST tag but delay aggregation, GST-htt-exon1(46Q) fusion proteins were exposed to factor Xa for $1 \mathrm{~h}$ on ice. Following this treatment, htt-exon1(46Q) was incubated with SUMO1, SUMO activation enzyme 
E1, SUMO E2 enzyme (Ubc9), and $\mathrm{Mg}^{2+}$-ATP for $1 \mathrm{~h}$ at $37^{\circ} \mathrm{C}$. SUMOylation was carried out in a 1.5:4 molar ration of SUMO1 to htt-exon1(46Q). For incubation and CD experiment, SUMOylation was carried out with a htt concentration of $4 \mu \mathrm{M}$. For ThT, PDA, and in situ AFM experiments, the SUMOylation step was performed with $8 \mu \mathrm{M}$ htt to take into account the required further dilutions steps associated with those assays, which were performed at a final concentration of $4 \mu \mathrm{M}$. Htt-exon1(46Q) used for unmodified controls was treated in the same manner but without the addition of SUMO1.

SUMOylation of htt was verified via Western blot analysis. Following the SUMOylation step, aliquots of SUMOylated htt, unmodified htt, and htt in the presence of mutant SUMO! were resuspended in sodium dodecyl sulfate (SDS) dissociation buffer, heated at $90^{\circ} \mathrm{C}$ for $5 \mathrm{~min}$, and loaded into polyacrylamide gels. The volume of aliquots was taken to contain $2 \mu \mathrm{g}$ of SUMO1. After SDS-PAGE, proteins were electro-transferred to a nitrocellulose membrane $(0.45 \mu \mathrm{m})$. The membranes were treated with blocking reagent and incubated with an anti-SUMO1 antibody (Enzo, 1:1000 dilution) overnight at room temperature. Blots were washed 3 times with TBST for 10 min before incubation with secondary Goat anti-mouse or anti-Rabbit Alkaline phosphatase antibodies (Millipore Sigma, 1:1000 dilution). After 3 washes for $10 \mathrm{~min}$ in TBST, the blots were developed by NBT/BCIP solution.

\subsubsection{Thioflavin-T Fluorescence Assav (ThT)}

ThT aggregation assays were conducted in a 384-well plate format using a microplate reader (SpectraMax M2). Stock ThT was prepared in ultrapure water at $1.0 \mathrm{mg} / \mathrm{mL}$. The final ThT concentration in each well was $0.06 \mathrm{mg} / \mathrm{mL}$. The aggregation of unmodified htt-exon1(46Q) with or without free SUMO and SUMOylated htt-exon1(46Q) was determined at a final concentration 
of $4 \mu \mathrm{M}$. The fluorescence intensity was measured using $440 \mathrm{~nm}$ excitation and $484 \mathrm{~nm}$ emission wavelength with $5 \mathrm{~min}$ interval for $15 \mathrm{~h}$ at $37^{\circ} \mathrm{C}$. All experiments were performed in triplicate.

\subsubsection{Circular Dichroism (CD) Spectroscopy}

To prepare samples for CD measurement, GST-htt-exon1(46Q) fusion proteins were dialyzed against Tris buffer $(20 \mathrm{mM}, \mathrm{pH} 7.3)$ for $24 \mathrm{~h}$ at $4{ }^{\circ} \mathrm{C}$ before cleavage of GST by factor Xa. Httexon1(46Q) final concentration $4 \mu \mathrm{M}$ for both unmodified and modified samples. Solutions of SUMO1, enzymes, and ATP were prepared to determine the background signal. As the solutions provided by the SUMOylation kit caused a large increase in the high-tension voltage, an additional dialysis step was required to obtain CD spectra. Background solutions required $24 \mathrm{~h}$ of dialysis to reduce the high-tension voltage to appropriate levels. As a result, spectra were obtained at $1 \mathrm{~h}$ after addition of the factor Xa for an unmodified htt-exon1(46Q) sample (which did not require the extra dialysis step) and after $24 \mathrm{~h}$ of incubation in a mini dialysis kit for unmodified and SUMOylated htt-exon1(46Q) samples. Samples were transferred to a $1 \mathrm{~mm}$ quartz cell, and CD spectra were obtained using a JASCO J-810 spectrometer with $50 \mathrm{~nm} / \mathrm{min}$ scanning speed and $0.1 \mathrm{~nm}$ data pitch at $20{ }^{\circ} \mathrm{C}$. Five spectra were collected between 190 to $260 \mathrm{~nm}$ for each sample and averaged together. Deconvolution of spectra was perfumed using DICHROWEB. (http://dichroweb.cryst.bbk.ac.uk/html/home.shtml) ${ }^{86}$

\subsubsection{Filter Retardation and Dot Blot Assay}

Following the SUMOylation procedure, $10 \mu \mathrm{M}$ solutions containing either unmodified httexon1(46Q) or SUMOylated htt-exon1(46Q) were incubated at $37^{\circ} \mathrm{C}$. As a positive control for fibril formation, a $20 \mu \mathrm{M}$ solution of unmodified htt-exon1(46Q) was also incubated. After 1, 3, 5,8 , and $12 \mathrm{~h}$ of incubation, $2.5 \mu \mathrm{g}$ of htt was removed from each solution and boiled in $300 \mu \mathrm{L}$ of $0.5 \%$ of sodium dodecyl sulfate (SDS) loading buffer for $5 \mathrm{~min}$ and stored at room temperature. 
After prewetting a nitrocellulose membrane $(0.45 \mu \mathrm{m}$ pore size $)$ with TBS for $5 \mathrm{~min}$, a Manifold slot blot apparatus (GE Healthcare) was assembled and connected to a vacuum. Each slot was washed with $300 \mu \mathrm{L}$ of TBS and allowed to run through the membrane under vacuum. Each 300 $\mu \mathrm{L}$ sample was pulled through the membrane in individual slots. The membrane was removed from the manifold, soaked in blocking buffer at $4{ }^{\circ} \mathrm{C}$ overnight, and incubated with either the httspecific monoclonal antibody MW8 (Developmental Studies Hybridoma Bank, University of Iowa, 1:350 dilution) or a SUMO1 antibody (Enzo, 1:1000 dilution) at room temperature for $1 \mathrm{~h}$. After washing with TBS, the membranes were incubated with secondary Goat anti-mouse or antiRabbit Alkaline phosphatase antibodies at a 1:1000 dilution. Following further washes, the membrane was incubated in BCIP/NBT substrate for 15 min to allow color development to verify the specificity of the MW8 antibody and the anti-SUMO antibody. Dot blots were also performed by spotting $2 \mu \mathrm{L}$ of incubated ( $8 \mathrm{~h}$ ) solutions of SUMO1, htt-exon1(46Q), and SUMOylated httexon1(46Q) directly onto a nitrocellulose membrane.

\subsubsection{Atomic Force Microscopy (AFM)}

All AFM experiments were performed with a Nanoscope V Multimode atomic force microscope (Veeco, Santa Barbara, CA). For ex situ AFM experiments, images were obtained in the tapping mode with diving-board-shaped silicon-oxide cantilevers with a nominal spring constant of $\sim 40$ $\mathrm{N} / \mathrm{m}$ and resonance frequency of $\sim 300 \mathrm{kHz}$ ( $\mu$ Masch). Samples were prepared by taking $3 \mu \mathrm{L}$ aliquots of each sample and deposited on freshly cleaved mica, allowed to set for 1 min, then washed with $200 \mu \mathrm{L}$ of ultrapure water, and dried with a gentle stream of air. For in situ AFM experiments, a tapping mode fluid cell with an $\mathrm{O}$ ring was equipped with a rectangular-shaped silicon nitride cantilever (Vista Probes) with a spring constant of $0.1 \mathrm{~N} / \mathrm{m}$. Lyophilized TBLE (Avanti Polar Lipids, Alabaster, AL) was reconstituted in $25 \mathrm{mM}$ Tris to a concentration of 1 
$\mathrm{mg} / \mathrm{mL}$. Using liquid nitrogen, 10 freeze-thaw cycles were performed to form bilayer sheets. Prior to experimentation, the lipid samples were bath sonicated for $10 \mathrm{~min}$ to promote lipid vesicle formation. TBLE vesicles ( $30 \mu \mathrm{L}$ of a $1 \mathrm{mg} / \mathrm{mL}$ solution) were injected directly into the fluid cell and allowed to fuse into a supported lipid bilayer on mica. The formation of the bilayer was monitored by continuous AFM imaging until a $30 \mu \mathrm{m} \times 30 \mu \mathrm{m}$ bilayer free from holes was formed (Fig 2.1).
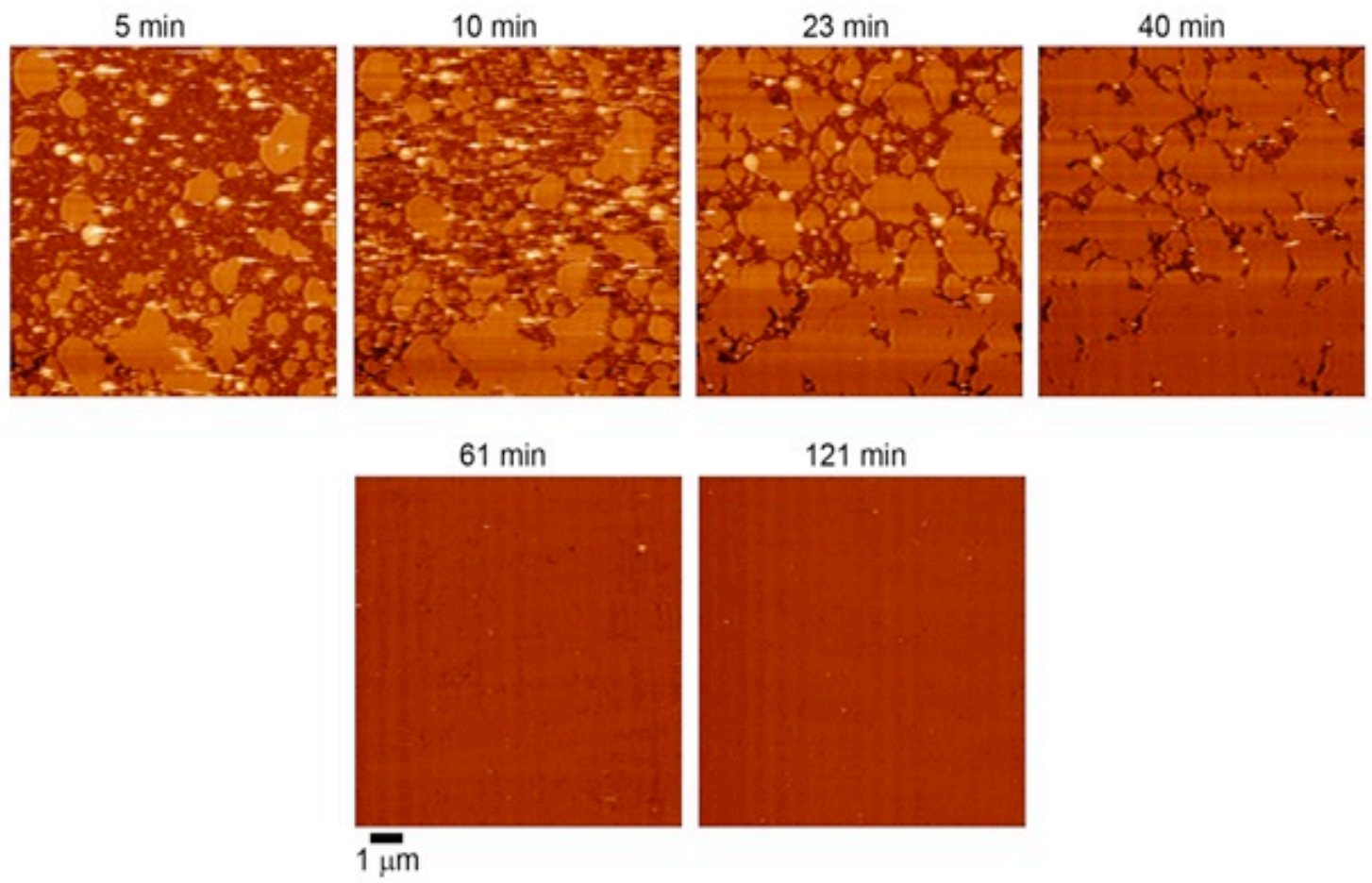

Fig 2.1 Time resolved in situ AFM images of a TBLE bilayer forming

Once the bilayer formed, $30 \mu \mathrm{L}$ samples were injected directly into the fluid cell to achieve a final htt concentration of $4 \mu \mathrm{M}$ in the cell. Aggregation on the lipid bilayer was monitored by continuous imaging. Images were processed utilizing Matlab equipped with the image processing toolbox (MathWorks, Natick, MA) as described. ${ }^{87}$ Images were flattened to correct for background curvature. To automatically identify individual aggregates, AFM images were converted into 
binary maps based on a height threshold, and clusters of pixels were identified with a recognition algorithm. Physical features of individual aggregates (e.g., height, volume, diameter, shape factor, length, aspect ratio, length, and area covered) were determined.

\subsubsection{TBLE/PDA Lipid Vesicle Protein Binding Assay}

TBLE/polydiacetylene (PDA) lipid binding assays were performed as described. ${ }^{88}$ TBLE and 10, 12 tricosadiynoic acid (Fisher Scientific) were mixed in a 2:3 molar ratio in $4 \mathrm{~mL}$ of ethanol/chloroform (1:1). The solution was dried under a nitrogen stream for 5 min, leaving a thin, dry film. Films were reconstituted in $8 \mathrm{~mL}$ of $25 \mathrm{mM}$ Tris buffered saline at $70{ }^{\circ} \mathrm{C}$. The solution was sonicated for $5 \mathrm{~min}$ at $100 \mathrm{~W}$ using a sonic dismembrator (Fisher Scientific). The suspension was cooled and stored at $4{ }^{\circ} \mathrm{C}$ overnight to allow for self-assembly of vesicles. The diacetylene monomers were polymerized by irradiation at $254 \mathrm{~nm}$ with a UV lamp with stirring to obtain a blue PDA/vesicles solution. The vesicles solution was covered with foil and stored in $4{ }^{\circ} \mathrm{C}$ prior to experiment. $50 \mu \mathrm{L}$ of $8 \mu \mathrm{M}$ unmodified htt, SUMOylated htt-exon1(46Q), and unmodified htt in the presence of free SUMO were mixed with $50 \mu \mathrm{L}$ of PDA solution in a 96 well plate format, resulting in a final htt concentration of $4 \mu \mathrm{M}$. All experimental conditions were performed in triplicate on each plate. The $\%$ colorimetric response (\%CR) of each well was recorded over $5 \mathrm{~h}$ with 5 min between readings using a microplate reader (SpectraMax M2) at $25{ }^{\circ} \mathrm{C}$ based on the

following equation: $\% C R=\left(\frac{P B_{0}-P B}{P B_{0}}\right) \times 100(1)$ where $\mathrm{PB}$, defined by $\mathrm{A}_{\text {blue }} /\left(\mathrm{A}_{\text {blue }}+\mathrm{A}_{\text {red }}\right)$, was calculated for the control $\left(\mathrm{PB}_{0}\right)$ and each sample condition $(\mathrm{PB})$. A $\mathrm{Allue}_{\text {is }}$ is the absorbance measured at $640 \mathrm{~nm}$, and $\mathrm{A}_{\text {red }}$ is the absorbance measured at $500 \mathrm{~nm}$. The negative control included equal ratios of $\mathrm{PDA} /$ lipid solution and buffer, while the positive control included saturated $\mathrm{NaOH}(\mathrm{pH}=$ 12) to create a range of colorimetric response for each lipid system. To correct for residual signal 
associated with free SUMO1, all experiments that included SUMO1 used a corresponding control SUMO1 only reaction as $\mathrm{PB}_{0}$.

\subsection{Results}

\subsubsection{Htt-exon1(460) is readily SUMOylated in Vitro}

Htt-exon1(46Q) Is Readily SUMOylated in Vitro. Experiments presented in this study were performed with a mutant htt fragment that expresses exon 1 with 46 repeats of the polyQ domain (htt-exon1(46Q)), which was purified from Escherichia coli as a soluble fusion with glutathione Stransferase (GST). Addition of factor Xa protease cleaves the GST moiety and initiates aggregation. However, an additional step to SUMOylate htt was required. To achieve SUMOylation of htt-exon1(46Q), cleavage of the GST moiety by factor Xa was performed for 1 $\mathrm{h}$ on ice followed by a SUMOylation reaction at $37^{\circ} \mathrm{C}$ for $1 \mathrm{~h}$. Due to limitations associated with the concentration of SUMO1 stock solutions, optimization of the SUMO reaction, and concentrations of htt-exon1(46Q) that would form fibrils in a reasonable time frame, the ratio of SUMO1 to htt-exon1(46Q) was 1.5:4. For aggregation experiments, we considered the completion of the SUMOylation step to be the $0 \mathrm{~h}$ reference. The extent of SUMOylation under these conditions was evaluated via Western blot analysis ${ }^{89}$ with primary antibodies specific for SUMO1 (Fig 2.2). SUMO with no htt appeared as a single band at $17 \mathrm{kDa}$ when detected with the SUMO antibody. A mutant SUMO1 protein, supplied with the SUMOylation kit, that does not bind to substrates was used as a control by mixing it with htt under SUMOylating conditions. The mutation in the SUMO1 proteins was a point mutation of the C-terminal amino acid from glycine to alanine, which disrupts the GG motif of SUMO1 that plays a role in the SUMOylation process. The mutant SUMO protein appeared as a single band at $17 \mathrm{kDa}$, demonstrating that nonspecific binding of SUMO to htt did not occur to an appreciable extent. When htt-exon1 was added to SUMO1 and 
appropriate enyzmes, bands of higher molecular weight were detected by the SUMO antibody, and these bands corresponded to bands in the WB correlating with htt-exon1(46Q) detected with MW1. Due to ambiguity associated with some of the htt bands, densitometry was performed on the WB stained with the SUMO antibody to determine the efficiency of the SUMOylation reaction. By comparing the density of bands that correlated with htt to the total SUMO signal, it was determined that $69-80 \%$ of SUMO1 was successfully conjugated to htt. Taking into account the molar ratio of SUMO to htt-exon1(46Q), this indicates that 15-20\% of htt-exon1(46Q) was SUMOylated.

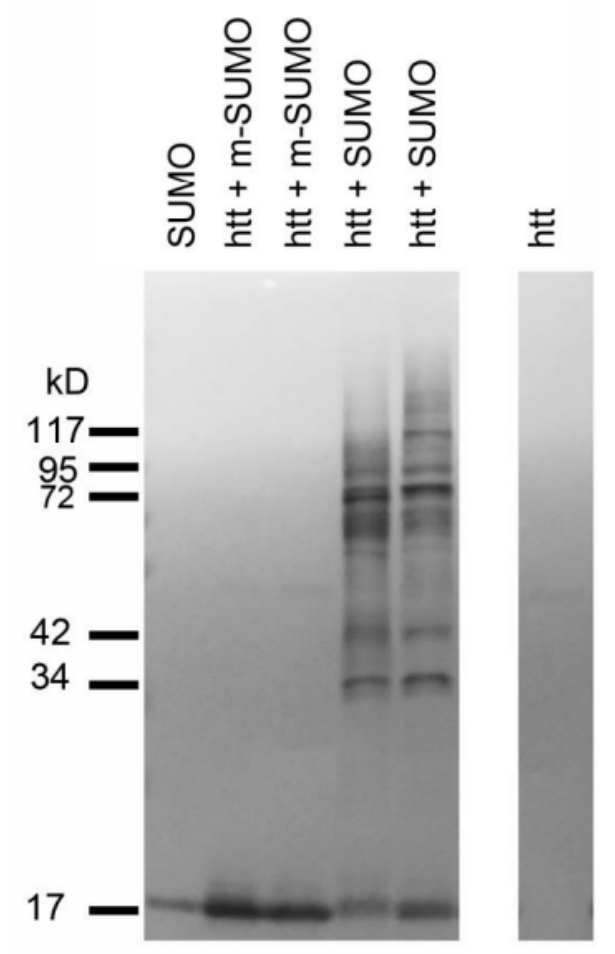

Fig 2.2 Western blot analysis of the SUMOylation of httexon1(46Q) using an anti-SUMO antibody. Control interactions were performed with a mutant SUMO protein that cannot be conjugated to lysine residues.

\subsubsection{SUMOylation inhibits htt-exon1 fibrils formation in Vitro and alters aggregate} morphology

Having established conditions to SUMOylate a fraction of htt-exon1(46Q), the impact of SUMOylation on htt aggregation was investigated with a Thioflavin T (ThT) fluorescence assay 
(Fig 2.3). ThT fluorescence is typically associated with binding $\beta$-sheet rich structures and is used to track fibril formation of amyloid-forming proteins. ${ }^{90}$ Three conditions were tested: unmodified htt-exon1(46Q), unmodified httexon1(46Q) with free SUMO1 (no enzymes were added to covalently bind SUMO1 to htt), and SUMOylated htt-exon1(46Q). As SUMO1 contains $\beta$-sheet content, an additional control experiment was performed with SUMO1, all of the conjugating enzymes, and ATP. These samples did not invoke a ThT signal.

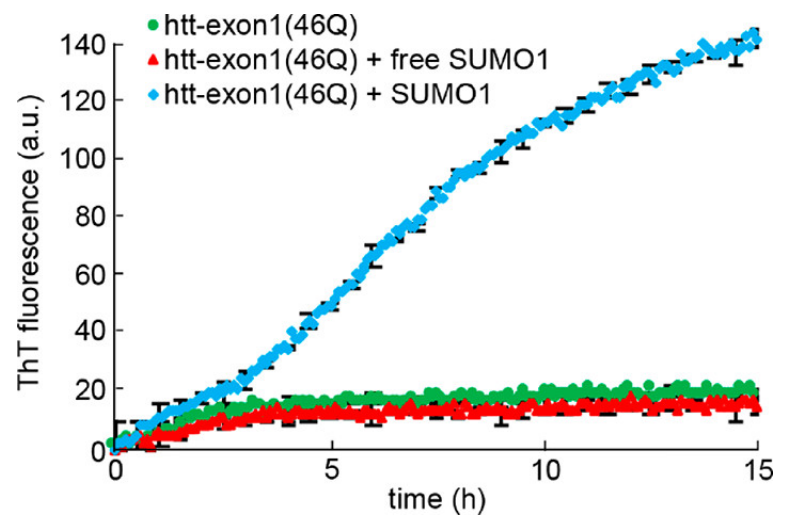

Fig 2.3 ThT analysis of unmodified and SUMOylated httexon1(46Q) aggregation. Aggregation of unmodified htt-exon1(46Q), unmodified htt-exon1(46Q) with free SUMO1 (unconjugated to htt), and SUMOylated htt-exon1(46Q) was monitored by ThT fluorescence. The concentration of htt-exon1(46Q) was $4 \mu M$. Error bars are provided for every 6th data point $(0.5 \mathrm{~h})$ and represent the SEM over three independent experiments.

Unmodified htt-exon1(46Q) induced a steady increase in ThT fluorescence for the first $3 \mathrm{~h}$, at which point a steady-state fluorescence signal was achieved. Incubations of free SUMO1 with unmodified htt-exon1(46Q) exhibited a similar aggregation trend that was not significantly different from unmodified htt alone. Upon SUMOylation of htt-exon1(46Q), the ThT signal was significantly larger than that associated with unmodified htt-exon1(46Q) and steadily increased over the entire time-course of the experiment, never reaching a steady-state value. This analysis suggests that SUMOylation enhances fibril formation. However, ThT signal intensity varies based on the exact morphology of fibrils, ${ }^{91}$ and a variety of nonamyloid structures and cavities can also 
invoke ThT fluorescence. ${ }^{92}$ As a result, further experiments are needed to verify the extent of aggregation associated with SUMOylation.

To further characterize the impact of SUMOylation on htt aggregation as a function of time, $4 \mu \mathrm{M}$ solutions of unmodified htt-exon1(46Q), free SUMO1 with unmodified httexon1(46Q), and SUMOylated htt-exon1(46Q) were incubated at $37^{\circ} \mathrm{C}$. Aliquots were taken from these incubations $1,3,5$, and $8 \mathrm{~h}$ after the SUMOylation reaction was performed, deposited on mica, and imaged via AFM (Fig 2.4A).

A $1 \mathrm{~h}$
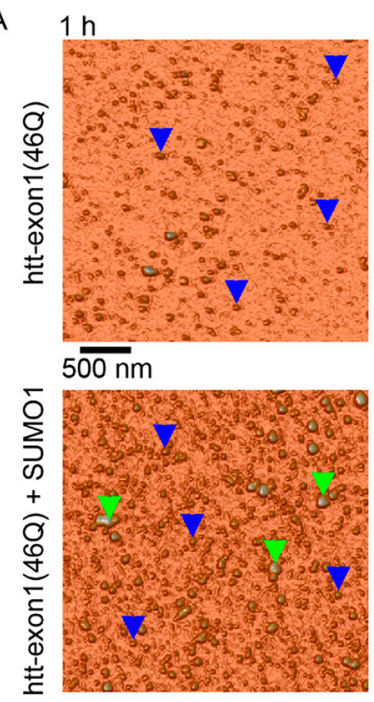

$3 \mathrm{~h}$
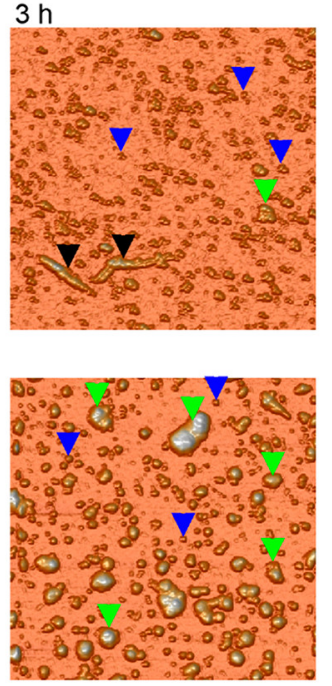
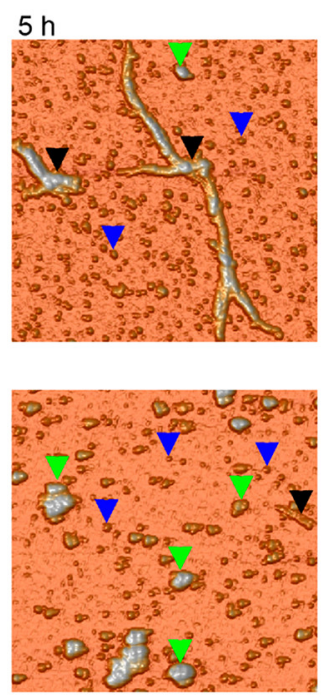
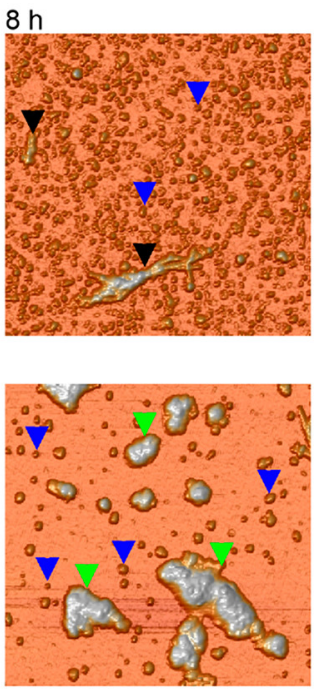
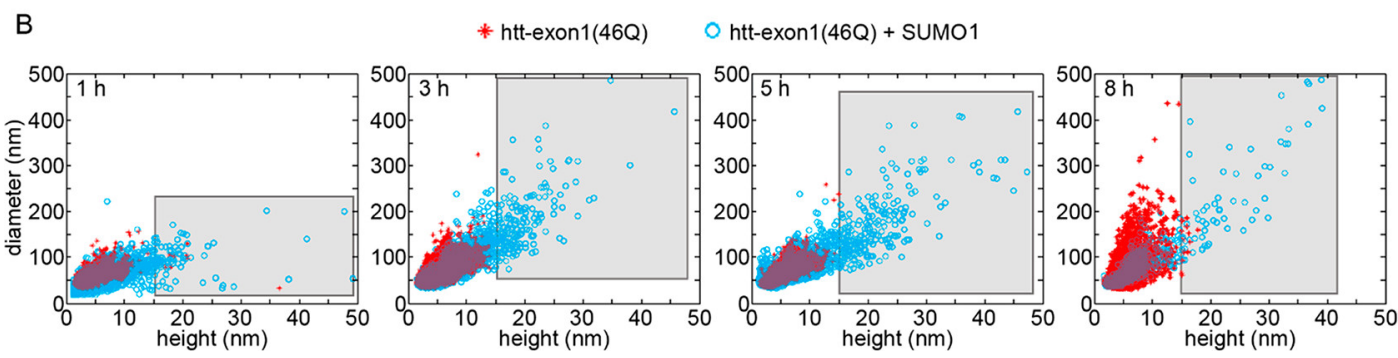

Fig 2.4 Ex situ AFM images of unmodified and SUMOylated htt-exon 1(46Q). (A) Representative AFM images of unmodified and SUMOylated htt sampled after 1, 3, 5, and $8 \mathrm{~h}$ of incubation. Examples of oligomers (blue arrows), fibrils (black arrows), and amorphous aggregates (green arrows) are indicated. These examples are based on the criteria described in the text. (B) Correlation plots of average diameter $v s$ height of all observed aggregate species. Shaded gray boxes indicating a population of aggregates of SUMOylated htt-exon1(46Q) that have a unique combination of morphological features compared to unmodified htt-exon $1(46 Q)$ controls. 
For incubations of unmodified htt-exon1(46Q), small oligomeric aggregates were detected after 1 $\mathrm{h}$. After $3 \mathrm{~h}$ of incubation, a small population of short $(<500 \mathrm{~nm}$ in length) fibrils were present in addition to numerous oligomers. The number and size of fibrils appeared to increase with time (5 and $8 \mathrm{~h}$ time points). Similar aggregation patterns and aggregate morphologies were observed for incubations of free SUMO1 with unmodified htt (Fig 2.5), and these observations are consistent with these two conditions invoking similar ThT responses. When htt-exon1(46Q) was SUMOylated, a large population of oligomers was observed after $1 \mathrm{~h}$ of incubation; however, fibrils did not abundantly form with time. Rather, SUMOylation of htt-exon1(46Q) promoted the formation of large, amorphous aggregates that steadily became larger with longer incubation. This observation is not consistent with the ThT assay if they are interpreted traditionally, i.e. that increased ThT signal is associated with fibril formation. Rather, this suggests SUMOylation may promote truncated $\beta$-sheet-rich oligomers that conglomerate into large, amorphous aggregation.

To evaluate and quantify the different aggregate species associated with unmodified and SUMOylated htt, morphological features of all the individual aggregates observed in the AFM images were measured with automated algorithms. To visualize the distribution of aggregate morphological features, correlation plots of aggregate height vs average diameter (average between the width and length of the bounding box associated with each aggregate) were constructed (Fig 2.4B). Based on these plots, there is a clear difference in aggregation patterns associated with htt-exon1(46Q) and SUMOylated htt-exon1(46Q). A distinct population of htt aggregates with heights greater than $15 \mathrm{~nm}$ formed from SUMOylated htt. The height of unmodified htt-exon1(46Q) aggregates does not appear to continue to increase; rather, the average diameter increases as elongated fibrils appear. In contrast, both the height and diameter of aggregates of SUMOylated htt continues to grow as amorphous aggregates grow larger in multiple 
directions. In contrast, comparison of correlation plots of unmodified htt-exon1(46Q) incubated with and without free SUMO1 demonstrates that these conditions result in aggregates of similar morphological characteristics (Fig 2.5B)
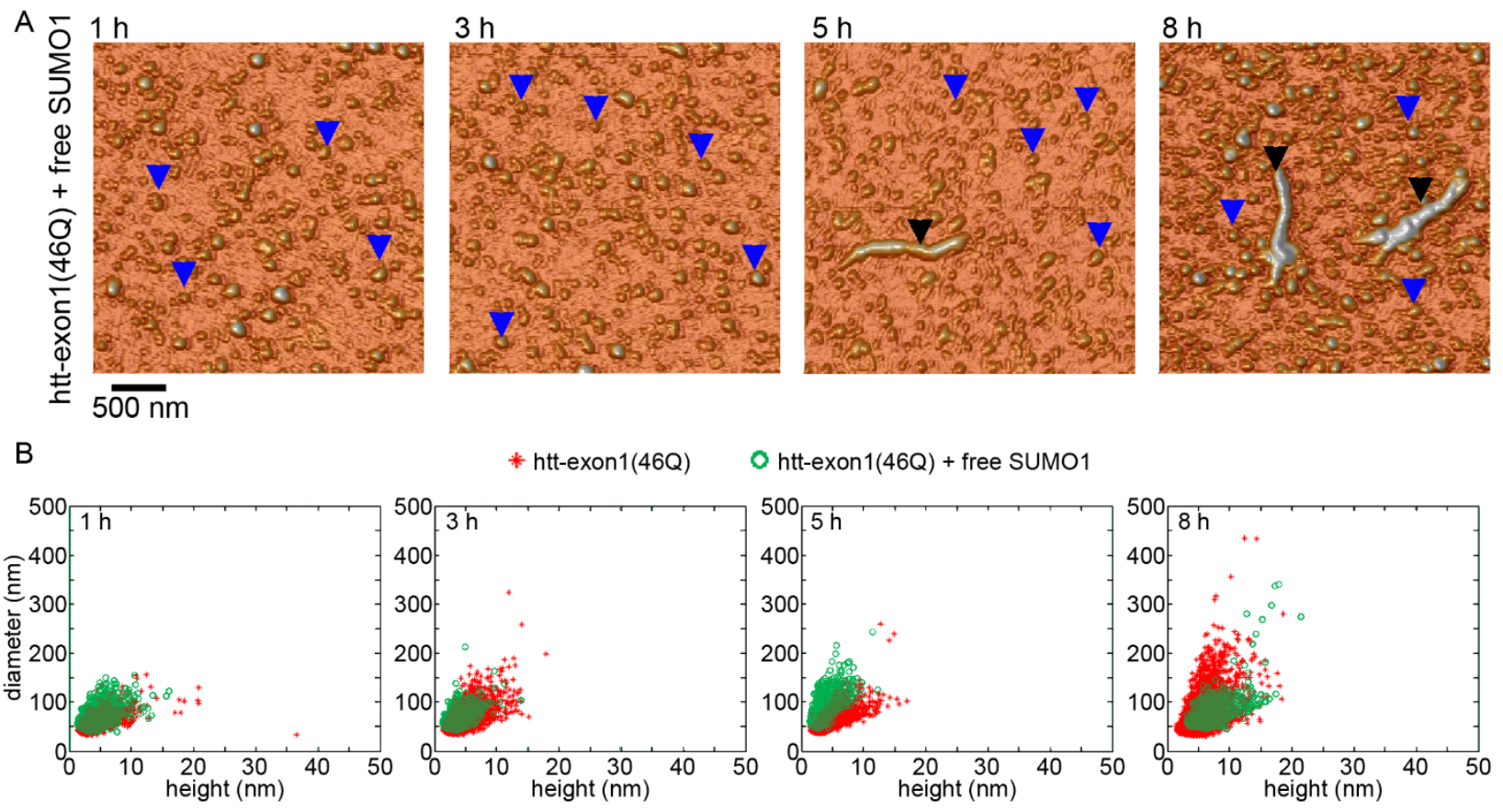

Fig 2.5 Ex situ AFM images of unmodified htt-exon 1(46Q) incubated with free SUMO1. (A) Representative AFM images of unmodified htt-exon1 (46Q) incubated with free SUMO1, that is the SUMOylation enzymes were not added, resulting in no measurable SUMOylation of htt-exon1(46Q). The incubation was sampled after 1, 3, 5, and $8 \mathrm{~h}$ and can be compared to the AFM aggregation assay provide in Fig 2.4 Examples of oligomers (blue arrows) and fibrils (black arrows) are indicated. (B) Correlation plots of average diameter vs height of all observed aggregate species compared to the unmodified htt-exon1 (46Q) control.

To quantify different aggregate species, present in AFM images for each incubation as a function of time, the number of oligomers, fibrils, and amorphous aggregates per $\mu \mathrm{m}^{2}$ was determined (Fig 2.6 and 2.7) by sorting each individual aggregate based on morphological features. In the past, we have been successful in sorting htt oligomers from fibrils by using an aspect ratio (longest distance across to shortest distance across) filter, ${ }^{87}$ however, this would not be sufficient to distinguish the amorphous aggregates from fibrils and/or oligomers. As a result, extra criteria and visual inspection was required. For any set of criteria, our algorithms place labels onto the 
AFM image to verify the ability to distinguish different aggregate species and allow for manual determination for the few aggregates with ambiguous assignments. For this analysis, oligomers were defined as any feature taller than $0.8 \mathrm{~nm}$ in height with an aspect ratio less than 2.5 (indicating a globular structure) and occupying less than $0.01 \mu \mathrm{m}^{2}$ of the surface. Amorphous aggregates were defined as being at least $0.8 \mathrm{~nm}$ in height and occupying a surface area greater that $0.01 \mu \mathrm{m}^{2}$. Fibrils were defined as aggregates taller than $0.8 \mathrm{~nm}$ in height with an aspect ratio greater than 2.5. As some aggregate species could meet the criteria for both amorphous aggregates and fibrils, differentiating between these ambiguous assignments required visual inspection of the labeled aggregate in each AFM image.

A

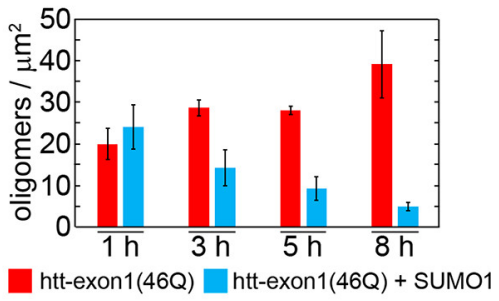

B
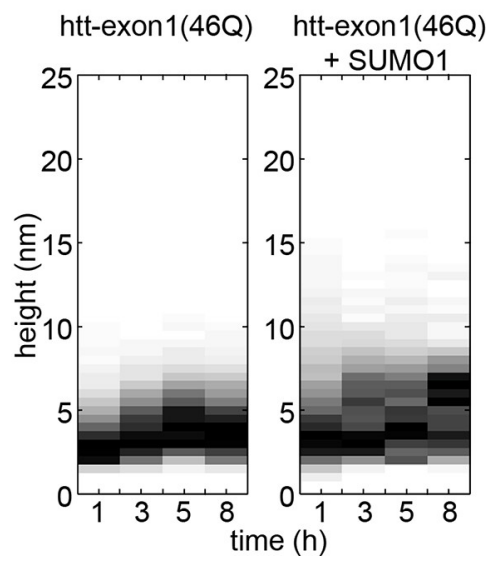

Fig 2.6 Analysis of oligomers observed in ex situ AFM images of unmodified and SUMOylated httexon1(46Q). (A) Number of oligomers (based on criteria provided in the text) per unit area observed as a function of time. Error bars represent the standard deviation. (B) Comparison of height histograms of all oligomers formed from unmodified and SUMOylated htt-exon1(46Q) as a function of time. Due to differences in the number of oligomers observed at different time points and conditions, each column is normalized by dividing the entire distribution by the number of oligomers contained in the most populated bin. 
After $1 \mathrm{~h}$ of incubation, a similar number of oligomers was observed for both httexon1(46Q) and SUMOylated htt-exon1(46Q) (Fig 2.6A), While the number of oligomers per unit area comprised of htt-exon1(46Q) gradually increased with time, SUMOylation of htt-exon1(46Q) resulted in a steady decrease in the oligomer population. Presumably, this decrease could be attributed to the formation of the large amorphous aggregates sequestering away smaller oligomers. Based on height histograms of all oligomers observed (Fig 2.6B), the initial (1 h time point) sizes of the oligomers comprised of unmodified htt-exon1(46Q) or SUMOylated httexon1(46Q) were similar (mode height of $\sim 2-3 \mathrm{~nm}$ ); however, there was a noticeable population of oligomers of SUMOylated exon1(46Q) larger than $7.5 \mathrm{~nm}(15.9 \%$ of the total observed oligomers) that was absent in the control htt incubations. With time, the size of unmodified httexon1(46Q) oligomers increased (mode height of $\sim 3-4 \mathrm{~nm}$ ), and some oligomers taller than 7.5 nm were observed $(4.1 \%, 6.0 \%$, and $5.1 \%$ of all observed oligomers for time points 3,5 , and $8 \mathrm{~h}$, respectively).

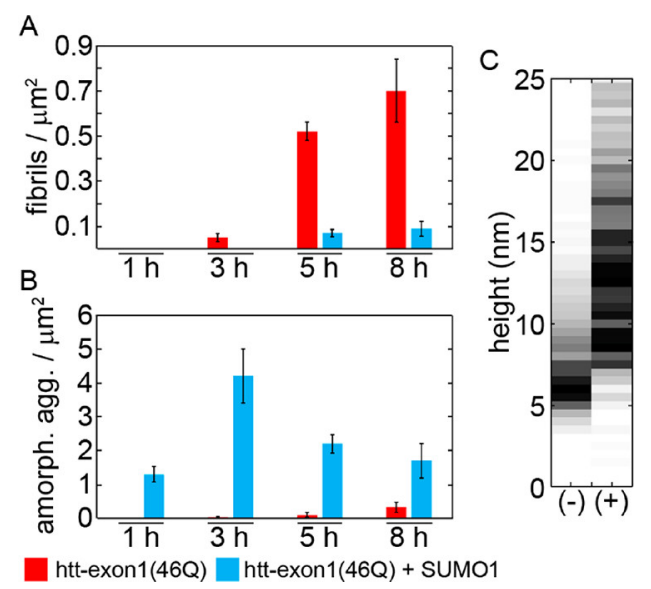

Fig 2.7 Analysis of fibrils and amorphous aggregates observed in ex situ AFM images of unmodified and SUMOylated htt-exon 1(46Q). (A) Number of fibrils (based on criteria provided in the text) per unit area observed as a function of time. (B) Number of amorphous aggregates (based on criteria provided in the text) per unit area observed as a function of time. Error bars in both (A) and (B) represent the standard deviation. (C) Comparison of height histograms of all fibrils and amorphous aggregates observed over the entire time frame of the AFM experiment formed from unmodified (-) and SUMOylated (+) httexon1(46Q). Due to differences in the number of fibrils and amorphous. 
For SUMOylated htt-exon1(46Q), the size of the oligomers increased to a greater extent, reaching a mode height of $\sim 5-7 \mathrm{~nm}$ by $8 \mathrm{~h}$ of incubation. There was also a prominent population of oligomers larger than $7.5 \mathrm{~nm}$ at these later time points for SUMOylated htt-exon1(46Q) (15.7\%, $15.6 \%, 14.7 \%$ of all oligomers for time points 3,5 , and $8 \mathrm{~h}$, respectively).

While unmodified htt-exon1(46Q) aggregated into fibrils, SUMOylation of httexon1(46Q) promoted larger amorphous aggregates (Fig 2.7). For unmodified htt-exon1(46Q), a small number of fibrils formed after $3 \mathrm{~h}$ of incubation, and this fibril population grew with time (Fig 2.6A). With SUMOylation, fibrils were not observed until after $5 \mathrm{~h}$ of incubation, and even then the population of fibrils was significantly smaller compared to incubations of unmodified htt. In contrast, a significant population of large amorphous aggregates formed in incubations of SUMOylated htt-exon1(46Q) as early as $1 \mathrm{~h}$ (Fig 2.7B). The raw number of amorphous aggregates peaked after $3 \mathrm{~h}$ for SUMOylated htt due to these aggregates coalescing into larger aggregates at 5 and $8 \mathrm{~h}$. Aggregates that fit the morphological criteria for amorphous aggregates were observed for incubations of unmodified htt-exon1(46Q), but the population and size of these aggregates was significantly smaller compared with SUMOylated htt. As both large fibrils and amorphous aggregates occupied larger surface areas, height histograms of all aggregates observed that occupied at least $0.01 \mu \mathrm{m} 2$ were compared for incubations of htt-exon1(46Q) and SUMOylated htt-exon1(46Q) (Fig 2.7C). As fibrils grow by addition of htt to their ends, the height of fibrils does not increase as they become longer, resulting in a tight distribution of height of $\sim 5.5-7.5 \mathrm{~nm}$. Such a distribution is observed for unmodified htt-exon1(46Q). In comparison, the amorphous aggregates grow in all directions, resulting in a broad, nonuniform distribution of height, as seen for SUMOylated htt-exon1(46Q). The contrast between the fibril and amorphous aggregate morphologies is further illustrated in (Fig 2.8), which compares AFM images and height profiles 
of the large aggregates observed for both conditions. Fibrils formed by htt-exon1(46Q) are typically less than $10 \mathrm{~nm}$ in their profiles; however, when fibrils cross or bundle, the height at that point can be slightly larger than $10 \mathrm{~nm}$. Amorphous aggregates are more variable in their height and can have regions with heights exceeding $20 \mathrm{~nm}$. However, these amorphous aggregates can be on the order of hundreds of nanometers in their lateral dimensions. Collectively, this analysis indicates that SUMOylation of htt promotes a broad size range of amorphous aggregates while it significantly interferes with the formation of fibrils which are typically observed for htt aggregation.

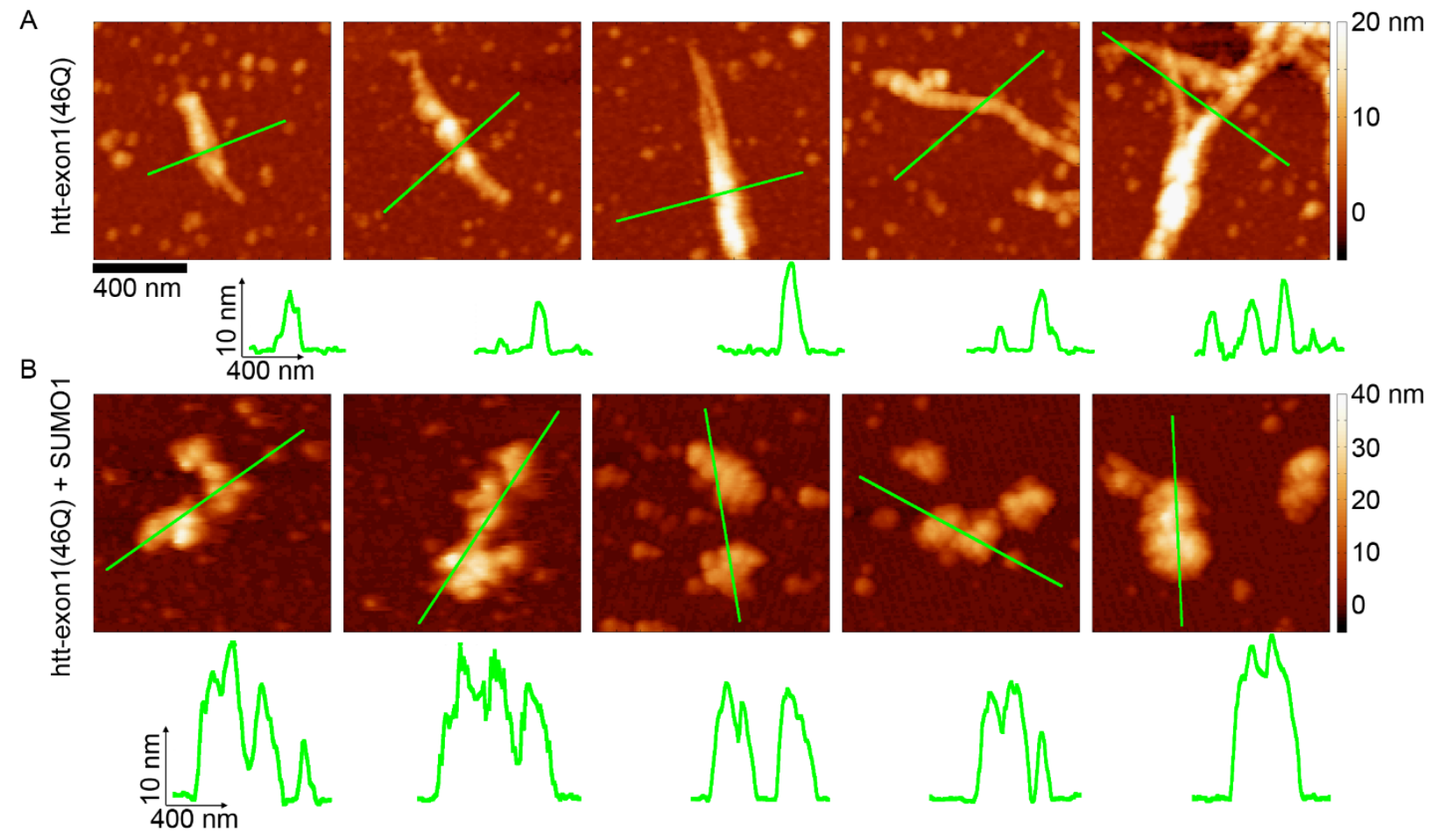

Fig 2.8 Representative AFM images demonstrating the different morphologies of (A) fibrils formed by unmodified htt-exon 1(46Q) and (B) amorphous aggregates of SUMOylated htt-exon1(46Q). The green line in each AFM image corresponds to the height profile directly below it. The scale bars in (A) and (B) for height profiles are identical.

\subsubsection{Secondary Structure of Aggregated htt Is Altered by SUMOylation}

Despite invoking a strong ThT signal, classic fibril morphology was not observed via AFM. We performed CD spectroscopy to glean information concerning the secondary structure associated 
with aggregation of unmodified and SUMOylated htt (Fig 2.9). Due to interference associated with the buffers used for SUMOylation, an additional $24 \mathrm{~h}$ dialysis step was required to obtain usable $\mathrm{CD}$ spectra. As a result, we were restricted to evaluating secondary structure after $24 \mathrm{~h}$ of incubation after SUMOylation. For unmodified htt-exon1(46Q) we were able to obtain CD spectra $1 \mathrm{~h}$ after the addition of factor Xa prior to performing the SUMO reaction.

Based on the CD spectra, unmodified htt-exon1(46Q) underwent a clear structural transition between 1 and $24 \mathrm{~h}$ of incubation (Fig 2.9A). Comparison of the CD spectra for unmodified and SUMOylated htt-exon1(46Q) after $24 \mathrm{~h}$ of incubation was clearly distinct. Deconvolution of the CD spectra (Fig 2.9B) suggests that htt-exon1(46Q) was comprised approximately of an equal mixture of $\alpha$-helical and coil structure. After $24 \mathrm{~h}$ of incubation, unmodified htt-exon 1(46Q) was predominately comprised of coil (61\%) with the helical content dropping to $2 \%$ of the secondary structure. Consistent with fibril $\beta$-sheet content increased to account for $37 \%$ of the secondary structure. In contrast to unmodified htt, SUMOylated htt retained a larger portion of its helical content (13\%). While coil still represented the largest secondary structural element (45\%), a large portion of $\beta$-sheet was also present (42\%). This suggests that the large amorphous aggregates contain significant $\beta$-sheet content.

While measured $\beta$-sheet content by $\mathrm{CD}$ spectroscopy was larger for aggregates of SUMOylated htt-exon1(46Q), it was not large enough to explain the large increase in ThT fluorescence compared with unmodified htt. This apparent discrepancy is plausibly due to a variety of factors. The amorphous aggregates indeed contain a significant amount of $\beta$-sheet structure to which ThT can bind. As the morphologies of aggregates are different for unmodified and SUMOylated htt, there could be variation in ThT affinity for these different aggregate types. Such a scenario is known for polymorphic fibrils of other amyloid forming proteins.${ }^{93} \mathrm{ThT}$ fluorescence 
can also arise from the binding of nonamyloid structures and cavities, ${ }^{92}$ which may be provided by the large amorphous aggregates associated with SUMOylation. Nevertheless, both ThT and CD analysis point to a large extent of $\beta$-sheet content in aggregates formed by SUMOylated htt despite the lack of traditional fibril morphologies.
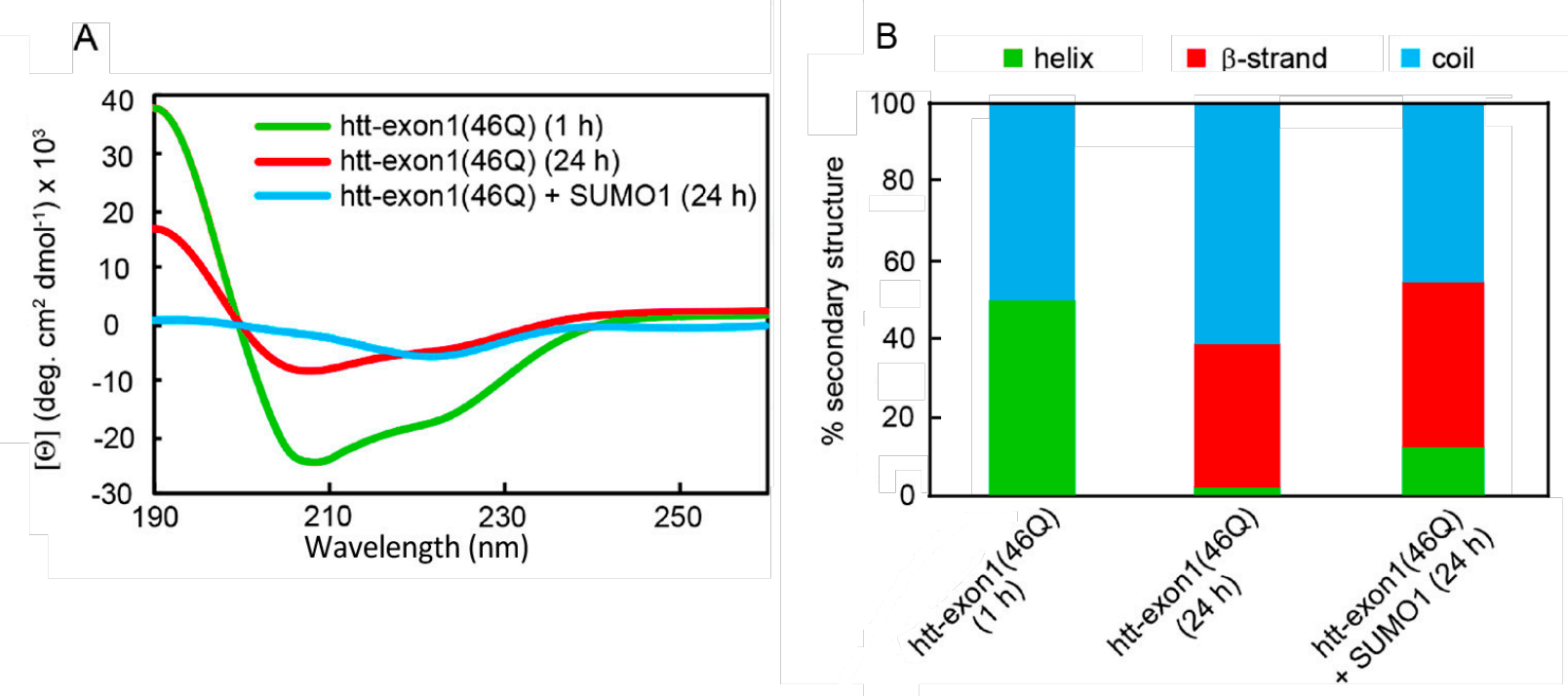

Fig 2.9 CD spectral analysis of secondary structural elements of aggregates of unmodified and SUMOylated htt-exon 1(46Q). (A) CD spectra were taken of $4 \mu M$ unmodified htt-exon1(46Q) after 1 and $24 \mathrm{~h}$ of incubation and $4 \mu M$ SUMOylated htt-exon 1(46Q) after $24 \mathrm{~h}$ of incubation. The $24 \mathrm{~h}$ time-point was required to allow for the removal, via dialysis, of elements associated with the SUMOylation buffers that interfered with the collection of the CD spectra. As a result, $C D$ spectra at intermediate time-points could not be collected. (B) Deconvolution of CD spectra was perfumed using DICHROWEB (http://dichroweb.cryst.bbk.ac.uk/html/home.shtml) to determine the content of $\alpha$-helical, $\beta$-strand, and coil secondary structural elements.

\subsubsection{SUMOylation Promotes the Formation of SDS Soluble Aggregates}

To further characterize how SUMOylation of Nt17 altered htt aggregation, unmodified and SUMOylated htt-exon1(46Q) was incubated (10 $\mu \mathrm{M})$, and aggregation was evaluated with a filter trap assay, which allows for the detection of SDS insoluble htt aggregates (predominately fibrils) that are too large to pass through a nitrocellulose membrane (pore size of $\sim 0.45 \mu \mathrm{m}$ ) under vacuum. In addition, a control incubation of $20 \mu \mathrm{M}$ htt-exon1(46Q) was also tested as a positive control for fibrillization. Captured fibrils were detected with the monoclonal htt antibody, MW8, 
which is known to recognize htt-exon 1 fibrils. ${ }^{94}$ The incubations $\left(37^{\circ} \mathrm{C}\right)$ of unmodified and SUMOylated htt-exon1(46Q) were sampled $(2.5 \mu \mathrm{g})$ after 1, 3, 5, 8, and $12 \mathrm{~h}$. For control incubations of htt-exon1(46Q) that had not been SUMOylated, SDS insoluble aggregates were detected after 5 and $8 \mathrm{~h}$ for 20 and $10 \mu \mathrm{M}$ solutions, respectively (Fig 2.11A). Based on the AFM analysis, these likely represent fibril aggregates. Once observed, the intensity of the MW8 staining increased over time, indicating an accumulation of fibrillar material on the membrane that is bigger than the pore size.

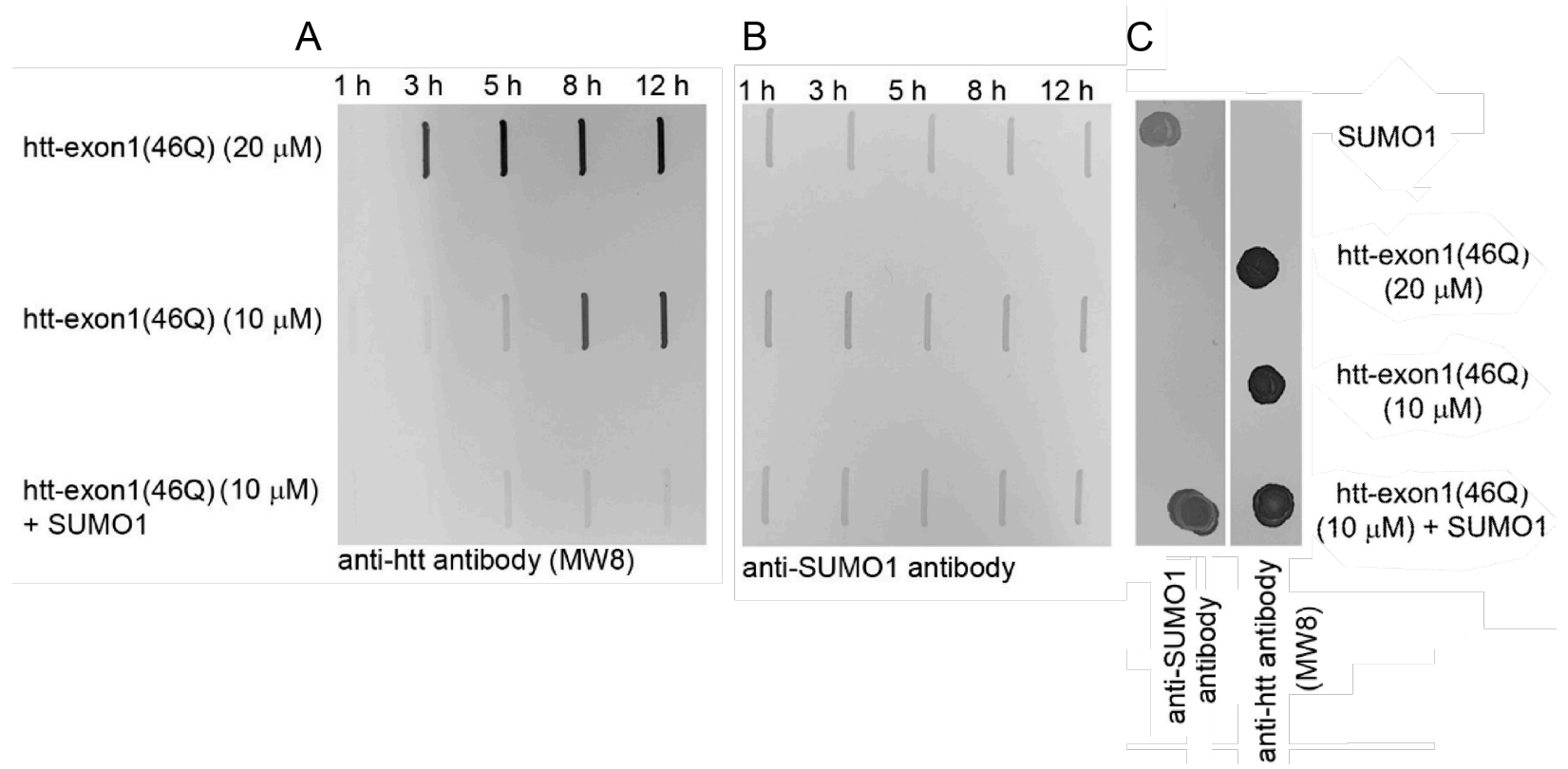

Fig 2.10 Immunochemical analysis aggregates formed by unmodified and SUMOylated htt-exon1(46Q). A filter retardation assay was performed on $10 \mu M$ incubations of unmodified and SUMOylated httexon1(46Q) aggregation reactions as a function of time. As a positive control for aggregation, incubations of $20 \mu \mathrm{M}$ htt-exon 1(46Q) were also analyzed. Membranes were probed for trapped (A) htt-exon 1(46Q) by $M W 8$ and (B) SUMO1 by SUMO specific antibody. (C) As a control to verify that MW8 and the anit-SUMO1 antibody could detect htt-exon 1(46Q) and SUMO1, a dot blot was performed on SUMO1, unmodified httexon1(46Q) (20 $\mu \mathrm{M}$ and $10 \mu \mathrm{M})$, and SUMOylated htt-exon1(46Q) (10 $\mu \mathrm{M})$ after $8 \mathrm{~h}$ of incubation.

When htt-exon1(46Q) was SUMOylated, the immunoreactivity with MW8 was absent at each time point (Fig 2.11A). Due to the observation of a significant population of large ( $>500 \mathrm{~nm}$ 
in diameter), amorphous aggregates via AFM, it is reasonable to hypothesize that these htt species would be trapped by the filter. However, there are two possible explanations for this observation: (1) that MW8 does not recognize the htt contained in these amorphous aggregates or (2) that these amorphous aggregates are dissolved by boiling in SDS. To distinguish between these two possibilities, the membrane was probed by a SUMO specific antibody provided by the SUMOylation kit (Fig 2.11B) because SUMO is likely incorporated into these large aggregates. No signal was detected for SUMOylated htt supporting the notion that these large, amorphous aggregates are dissolved by boiling in SDS.

To verify that MW8 and the anti-SUMO antibody could bind to the large amorphous aggregates and eliminate the possibility of a false negative result in the slot blot assay, a dot blot assay was performed (Fig 2.10C). Four different conditions were tested: SUMO1 alone, unmodified htt-exon1(46Q) (10 and $20 \mu \mathrm{M})$, and SUMOylated htt-exon1(46Q) (10 $\mu \mathrm{M})$. Samples were incubated for $8 \mathrm{~h}$ to allow for the formation of higher order aggregates before direct deposition on a nitrocellulose membrane and probed by MW8 and the anti-SUMO antibody. On the dot blot, MW8 detected unmodified and SUMOylated htt-exon1(46Q) samples, but not SUMO1 alone. The anti-SUMO antibody detected SUMO1 alone and SUMOylated htt but did not detect unmodified htt samples. This suggests that if the large amorphous aggregates of SUMOylated htt-exon1(46Q) were retained by the filter trap that they would have been detected by both MW8 and the anti-SUMO antibodies.

\subsubsection{SUMOylation Inhibits htt-Lipid Interaction}

Nt17 functions as a lipid binding domain. ${ }^{35,87}$ In an effort to understand how SUMOylation influences the interaction of htt-exon1 with lipid membranes via Nt17, we performed total brain lipid extract (TBLE)/polydiacetylene (PDA) vesicle lipid binding assays. This is a colorimetric 
assay that can temporally quantify peptide-membrane interactions and interfacial membrane processes. The mixed vesicles comprised of phospholipids (in this case TBLE) and polymerized PDA exhibit a visible color change (blue to red) induced by interactions between peptides and the phospholipid moieties within the vesicles due to transitions of the PDA polymer backbone structure. ${ }^{88,95}$ By measuring absorbance of both the blue $(640 \mathrm{~nm})$ and red $(500 \mathrm{~nm})$ wavelengths associated with the TBLE/PDA vesicles upon exposure to unmodified htt-exon1(46Q) with or without free SUMO1 or SUMOylated htt-exon1(46Q) (all at concentrations of $4 \mu \mathrm{M}$ ), the \%CR was obtained as a function of time (Fig 2.11A). Importantly, the calculation of the $\% \mathrm{CR}$ can be set up to take into account background experiments performed with SUMO1 in the absence of htt. To ensure that the htt-exon1(46Q) interacted with the TBLE/PDA vesicles, additional reactions were performed with unmodified htt at $20 \mu \mathrm{M}$. $\mathrm{NaOH}$ exposure served as a positive control to verify that the TBLE/PDA vesicles were functioning properly. The TBLE/PDA vesicles were also exposed to Tris buffer as a negative control.

Upon exposure to htt-exon1(46Q), the \%CR increases rapidly for $\sim 2-3 \mathrm{~h}$ until a steadystate interaction is achieved. As expected, the steady state \%CR was $3 \times$ larger for htt-exon1(46Q) at $20 \mu \mathrm{M}$ compared to htt at $4 \mu \mathrm{M}$ (Fig 2.11A). At the $4 \mu \mathrm{M}$ concentration, the steady-state response was $\sim 7-8 \%$, indicative of the interaction of htt-exon1(46Q) at $4 \mu \mathrm{M}$ with the lipid vesicles. Incubations of free SUMO1 with unmodified htt-exon1(46Q) result in a similar interaction the PDA/TBLE vesicle and were not notably different from unmodified htt alone. In contrast, SUMOylated htt $(4 \mu \mathrm{M})$ did not induce a rapid increase in the colorimetric response; rather, the $\%$ CR was steady at about $2 \%$ over $5 \mathrm{~h}$, suggesting that the SUMOylation completely inhibits the ability of htt-exon 1 to bind and aggregate on lipids. 
As the PDA assay suggested that SUMOylation abolished the interaction of htt-exon1 with TBLE vesicles, we next performed a series of in situ AFM experiments to further validate this observation. Supported TBLE bilayers on mica were prepared via vesicle fusion. Such supported bilayers have been previously used as model surfaces to study the interaction of several amyloidforming proteins with lipid membranes. ${ }^{96-99}$ From trial to trial, the total concentration of lipid in the AFM fluid cell was maintained, but observations were restrained to a $30 \times 30 \mu \mathrm{m}$ patch of bilayer that was verified to be defect-free (at the level of resolution of the AFM) prior to exposure to any further proteins. Such TBLE bilayers are smooth with an (RMS roughness about $0.4 \mathrm{~nm}$, Fig 2.11B) and stable under AFM imaging conditions (for at least $24 \mathrm{~h}$ ). To control for any influence of the SUMO protein alone on the integrity of the bilayer, control experiments were performed by exposing freshly formed TBLE bilayers to the SUMO protein, SUMO enzymes, and ATP solution at the same concentrations as would be used in experiments with htt. Following the injection of this SUMO solution into an AFM fluid cell, the bilayer morphology was unaffected for well over $5 \mathrm{~h}$ with no discernible effect on the bilayer roughness (Fig 2.11C).

Having established that SUMO alone did not alter TBLE bilayer morphology, TBLE bilayers were exposed to either unmodified htt-exon1(46Q) or SUMOylated htt-exon1(46Q) at a final concentration of $4 \mu \mathrm{M}$ in the fluid cell. The protein aggregation on the bilayer was directly monitored by continuous in situ AFM imaging for $5 \mathrm{~h}$ (Fig 2.11D). 

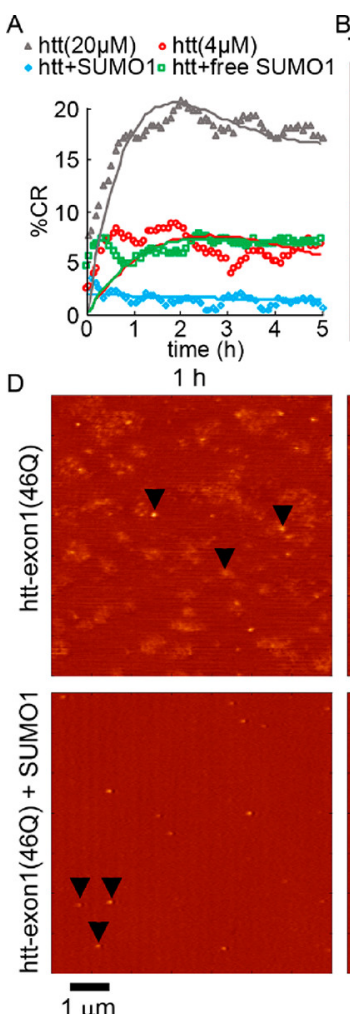

B TBLE alone

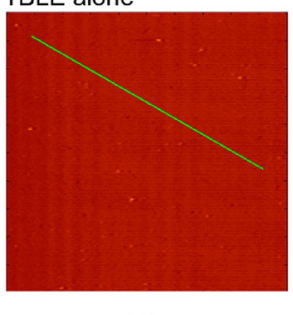

$2 \mathrm{~h}$
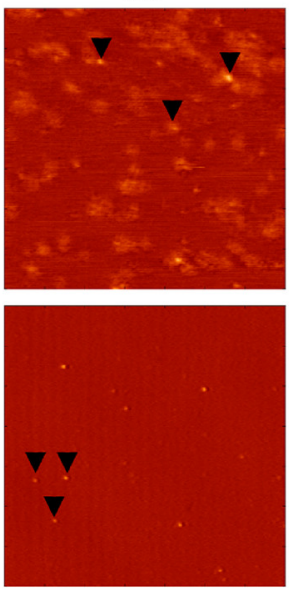

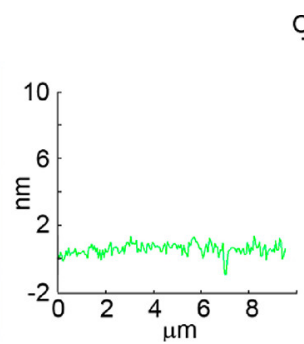

$3 \mathrm{~h}$
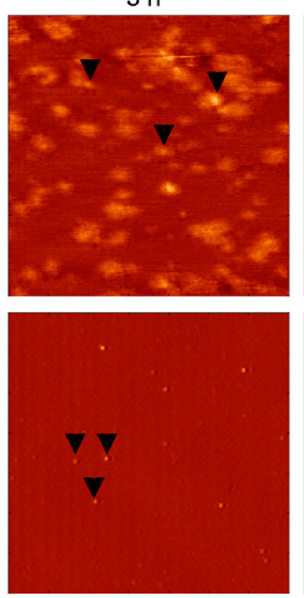
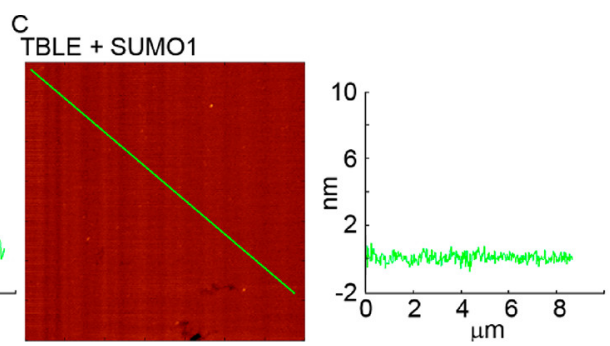

$5 \mathrm{~h}$
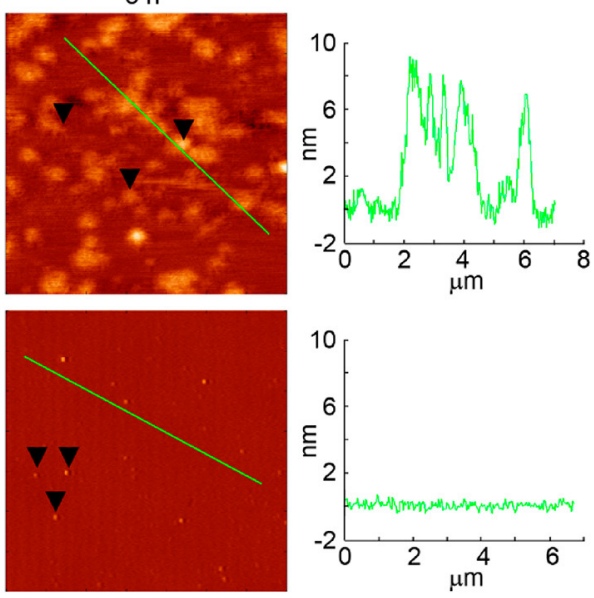

Fig 2.11 SUMOylation of htt-exon1(46Q) inhibits the ability of htt to interact with total brain lipid extract bilayers. (A) PDA/TBLE lipid vesicle binding assay of $4 \mu M$ unmodified htt-exon 1(46Q), free SUMO1 with unmodified htt-exon1(46Q), and SUMOylated htt-exon1(46Q). Positive control experiments included exposure of PDA/TBLE vesicles to $20 \mu M$ htt-exon1(46Q). (B) Freshly made TBLE bilayer observed by in situ AFM. The green line corresponds to the height profile provided to the right. (C) In situ AFM image of a TBLE bilayer exposed to SUMO1 for $5 \mathrm{~h}$. The green line corresponds to the height profile provided to the right. (D) Time-resolved AFM images of TBLE bilayers exposed to unmodified htt-exon1(46Q) and SUMOylated htt-exon 1(46Q). Arrows are provided to demonstrate common features in consecutive images, verifying that this is the same spot of the lipid bilayer. The green lines in the $5 \mathrm{~h}$ time point correspond to the height profile provided to the right.

Upon initial exposure of the TBLE bilayer to htt exon1(46Q), discrete patches of disrupted bilayer morphology (increased roughness) appeared that often contained features reminiscent of htt oligomers. Unlike the ex situ AFM experiment performed at the same htt concentration, fibrils were not observed on the lipid bilayer; however, the aggregates were globular in appearance and remained stable in consecutive images. With time, the area of the bilayer disrupted by exposure to htt increased, and the roughness associated with these regions appeared more pronounced. After 5 h of exposure to htt-exon1(46Q), $44 \pm 6 \%$ of the TBLE bilayer surface appeared to be disrupted. 
In contrast, TBLE bilayers that were exposed to SUMOylated htt-exon1(46Q) did not develop disrupted regions over the entire $5 \mathrm{~h}$ experiment. A few discrete oligomers did appear on the bilayer surface, but no large amorphous aggregates were observed, suggesting that SUMOylated htt and the resulting aggregates do not readily interact with TBLE bilayers. This observation is consistent with the PDA results implying the inhibitory function of SUMO conjugation on htt lipid binding.

To quantify the extent of disruption correlated with the interaction of lipid exposure with unmodified or SUMOylated htt, the RMS roughness of the different bilayers was measured (Fig 2.12). For the bilayers exposed to unmodified htt-exon1(46Q), two RMS roughness measurements were obtained: one of the patches exhibiting increased roughness (rough) and the other of the unaffected regions of the bilayer (smooth).

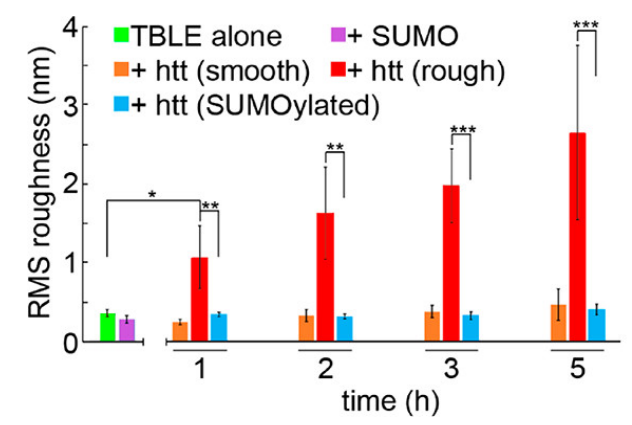

Fig 2.12 Analysis of the RMS roughness of in situ AFM images of TBLE bilayers exposed to unmodified or SUMOylated htt-exon 1(46Q). TBLE alone refers to the RMS roughness of lipid bilayers prior to exposure to any protein. + SUMO indicates the RMS roughness measured from bilayers exposed to SUMO1 for $5 \mathrm{~h}$. + htt (smooth) refers to the RMS roughness of regions of bilayers exposed to unmodified htt-exon1(46Q) that did not display altered morphology. + htt (rough) refers to the RMS roughness of regions of bilayers disrupted by unmodified htt-exon1(46Q). + htt (SUMOylation) refers to the RMS roughness of bilayers exposed to SUMOylated htt-exon 1(46Q). * indicates $p<0.05$, **indicates $p<0.01$, and $* * *$ indicates $p<$ 0.001 based on a t test $(n=3)$. Error bars represent standard deviations.

Control TBLE bilayers had an RMS roughness of $0.36 \pm 0.04 \mathrm{~nm}$, and TBLE bilayers that were only exposed to SUMO had an RMS roughness of $0.29 \pm 0.05 \mathrm{~nm}$. The smooth regions of bilayers exposed to htt-exon1(46Q) were comparable $(<0.5 \mathrm{~nm})$ at all time points, but the disrupted regions were significantly rougher $(>1.0 \mathrm{~nm})$ at all time points, and the roughness increased with time. The 
RMS roughness of bilayers exposed to SUMOylated htt was comparable to the controls over the entire time frame of the experiments, reaching a maximum RMS roughness of $0.41 \pm 0.7 \mathrm{~nm}$ after $5 \mathrm{~h}$.

\subsection{Discussion}

While the expansion of polyQ domains in proteins is the root cause of numerous diseases, ${ }^{18}$ the context of the polyQ domain within the proteins can profoundly influence the disease-related aggregation process. In the case of htt, the $\mathrm{Nt} 17$ and the polyP sequences directly adjacent to the polyQ domain impact aggregation, ${ }^{27,100,101}$ and both have been shown to be viable targets to inhibit htt fibrilization. ${ }^{30,94}$ Several PTMs (phosphorylation, acetylation, and oxidation) of the Nt17 sequence impact htt aggregation in vitro. ${ }^{35}$ In addition, SUMOylation of htt via the Nt17 domain has been shown to reduce insoluble htt aggregates in cellular models of HD. ${ }^{72,73,83,84}$ In this study, we investigated the impact of SUMOylation of a mutant htt-exon1 protein on its ability to aggregate and interact with lipid membranes.

While htt-exon1(46Q) readily aggregated into fibrils, SUMOylation promoted a distinct aggregation pathway that resulted in large, amorphous aggregates (Fig 2.13A). Both unmodified and SUMOylated htt formed oligomers, but based on morphological analysis, the oligomer species appear to be unique. Taking into account the ThT, AFM, and CD data, a mechanism for the SDS solubility of the amorphous aggregates formed by SUMOylated htt can be proposed. While only a small portion (15-20\%) of htt was successfully SUMOylated under our experimental conditions, it had a significant impact on aggregation. Unmodified htt-exon1 aggregates via an $\mathrm{Nt17}$ mediated $\alpha$-helix rich oligomer involved in nucleating fibril formation. ${ }^{14,101}$ A potential scenario for SUMOylation's impact on aggregation could be similar to the mechanism associated with the ability of free $\mathrm{Nt17}$ peptides (Nt17 peptides without an adjacent polyQ domain) to inhibit fibril 
formation. ${ }^{30}$ Free Nt17 peptides are incorporated into the $\alpha$-helical intermediates, decreasing the density of polyQ within the oligomer, reducing the efficiency of nucleation within the oligomer. ${ }^{30}$ SUMO1, being much larger than a free Nt17 peptide, could also create spacing between polyQ domains of htt, reducing fibril formation. However, the addition of Nt17 to htt-exon1(46Q) does not promote large amorphous aggregates; rather, it appears to stabilize a tight distribution of oligomers. ${ }^{37}$ While the Nt17 peptide is about $2.0 \mathrm{kDa}$, SUMO1 is an $11.5 \mathrm{kDa}$ protein. Yet, the formation of $\beta$-sheet structure by SUMOylation under our conditions suggests that nucleation was not inhibited. This could be due to only $15-20 \%$ of htt being SUMOylated, as inhibition of htt aggregation of Nt17 was also dose dependent. As SUMOylated htt that is incorporated into oligomers would still contain $\beta$-sheet competent polyQ domains, it is also plausible that oligomers formed under our SUMOylated condition still facilitate intermolecular polyQ interactions resulting in $\beta$-sheet structure; however, the bulky SUMO1 proteins prevent, via steric hindrance, further addition of htt monomers required for fibril elongation. This would, in effect, create truncated $\beta$ sheet-rich oligomers. These stunted htt oligomers containing SUMO1 conglomerate into large amorphous aggregates; however, the presence of SUMO1 prevents the creation of the large-scale order that would be associated with standard fibril structures. As a result, the stability of these mixed amorphous aggregates would be compromised by the incorporation of SUMO1, making them susceptible to be broken apart into fibril-forming incompetent oligomers upon boiling in SDS. This scenario is consistent with the height of htt oligomers under SUMOylating conditions being similar to the height of htt fibrils, the enhanced ThT fluorescence associated with SUMOylated htt, and the development of $\beta$-sheet structure with SUMOylated htt as detected by CD. 
In addition, SUMOylation abolished the ability of htt-exon1 to associate with and disrupt TBLE bilayers (Fig 2.13B). As Nt17 facilitates the binding of htt-exon1 to lipid membrane, SUMOylation would provide steric hindrance to the binding interaction, but only a fraction of htt was effectively SUMOylated. In principle, the unmodified portion of htt-exon1 should have been available to interact with lipids unimpeded; however, SUMOylating 15-20\% of htt completely blocked lipid binding.

A

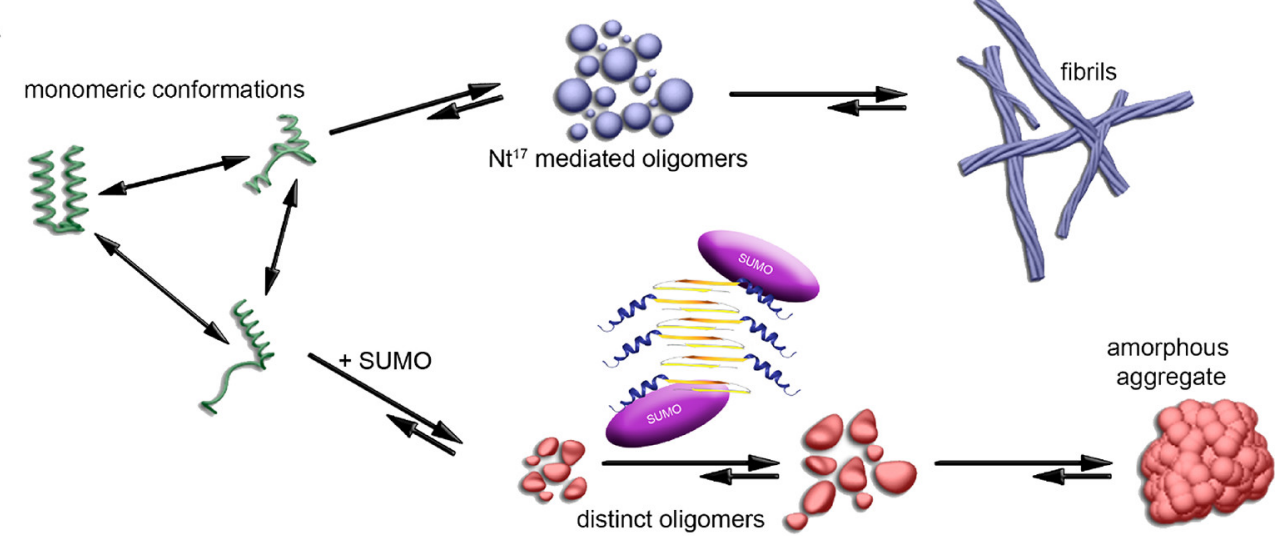

B

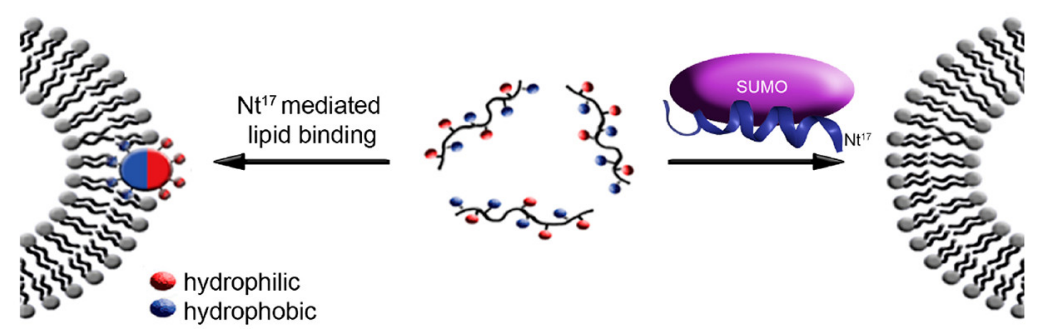

Fig 2.13 Cartoon summarizing the impact of SUMOylation on (A) htt-exon1 aggregation and (B) httexon1's ability to bind lipid membranes. (A) Htt-exon1 with expanded poly $Q$ domains samples a variety of monomeric conformations, some of which are aggregation prone. Aggregation of unmodified htt-exon1 proceeds through an $\alpha$-helical oligomeric intermediate that is mediated by the Nt17 domain on the way to forming amyloid fibrils. When a subpopulation of htt-exon1 is SUMOylated via Nt17, a distinct (compared to the unmodified condition) oligomer species forms likely due to the incorporation of the bulky SUMO constituent in comparison to the size of the Nt17 domain. These intermediates are likely $\beta$-sheet rich oligomers that are stabilized by steric hindrance associated with the incorporation of SUMO1. These oligomers tend to grow in size rather than transitioning to a fibrillar morphology, eventually coalescing into large amorphous aggregates that are SDS soluble. (B) Due to its ability to form an amphipathic $\alpha$ helix, Nt17 facilitates the binding of htt-exon1 to lipid membranes. Upon the addition of a bulky SUMO constituent, the ability of Nt17 to associate with lipid membranes is blocked. 
A potential mechanism for this is that htt oligomers have formed within the time required to perform the SUMOylation reaction. As a result, the control experiment using unmodified httexon1 is measuring the affinity of oligomers for lipid membranes rather than monomers. While oligomers of unmodified htt interact strongly with membranes, the inclusion of SUMO1 into htt oligomers prevents their ability to bind lipids. Collectively, these observations suggest that SUMOylation attenuates the aggregation process of htt and the ability of htt to interact with other physiologically relevant molecules and underscores the complexity of htt aggregation.

SUMOylation of amyloid-forming proteins is a common phenomenon; ${ }^{102-104}$ however, the impact of SUMOylation on the aggregation of these different proteins varies. For example, $\alpha-$ synuclein ( $\alpha$-syn) from the brains of transgenic mice is SUMOylated, ${ }^{105}$ and SUMOylated $\alpha$-syn is found in Lewy bodies in brains from patients with Parkinson's disease and dementia with Lewy bodies. ${ }^{106}$ Similar to the observed inhibition of htt fibrillization observed here, $\sim 50 \%$ of SUMOylation in $\alpha$-syn abolishes fibril formation, and only 10\% SUMOylation can significantly delay fibril formation. ${ }^{105}$ SUMO1 promotes SOD1 aggregation and colocalize into aggregates, ${ }^{107}$ and SUMO3 accelerates the aggregation of familial ALS-linked mutant SOD1 proteins. Enhanced SUMOylation is a common aspect of polyQ diseases. ${ }^{108}$ SUMOylation of Ataxin-7, another aggregating protein associated with expanded polyQ, stimulated the formation of insoluble aggregates; ${ }^{109,110}$ however, SUMOylation of Ataxin-3 stabilizes the protein, reducing aggregation. ${ }^{110,111}$ SUMOylation also plays a role in the processing of amyloid-forming proteins with various impacts on the disease state. APP processing linked to A $\beta$ production is modified by SUMOylation. ${ }^{112}$ SUMO also appear to modify Tau phosphorylation. ${ }^{113}$

While SUMO1 colocalized with htt inclusions, overall inclusions are reduced with SUMOylation of htt, htt stability was increased, and overall aggregation was reduced in 
cellular models of HD. ${ }^{72}$ While SUMOylation suppressed htt amyloid formation, it promoted httinduced neurodegeneration in a drosophila model of HD. ${ }^{72}$ Rhes, a small G protein family, specifically stimulates mutant htt SUMOylation but not wild-type. ${ }^{73}$ When mutant htt is SUMOylated by Rhes, reduced aggregation and enhanced cytotoxicity was observed. However, in both of these studies, ${ }^{72,73}$ aggregation was assessed by material that failed to enter a gel using SDSPAGE, and our analysis suggests that large, nonfibrillar aggregates comprised of SUMOylated htt may not be SDS stable. As a result, the distinct aggregate species we observe in vitro may not have been readily detected by these studies, and the enhanced toxicity associated with SUMOylation of htt may lie in the formation of these SDS-soluble aggregates. Modifying htt SUMOylation in the R6/2 mouse model of HD via reducing PIAS1, an htt-selective E3 SUMO ligase, results in altered phenotype. ${ }^{83,84}$ Increasing PIAS-1 improved behavioral phenotype; decreasing PIAS-1 exacerbated phenotype ${ }^{84}$ Inclusion formation may be a protective event, and the most toxic htt species, i.e. nanoscale oligomers and fibrils, remain diffuse throughout the cell. ${ }^{20-22,24,25}$ The increased cytotoxicity associated with SUMOylated htt may be due to the formation of these unique aggregates promoted by SUMOylation.

Many toxic mechanisms have been attributed to mutant htt, including nuclear DNA damage, ${ }^{68,114}$ transcription dysregulation, ${ }^{115,116}$ impairing function of other proteins via sequestration within aggregates, ${ }^{117,118}$ interference with central protein quality control and clearance mechanisms, ${ }^{119-121}$ alteration in endocytosis and microtubule-based transport, ${ }^{39}$ disruption of cellular/subcellular membranes, ${ }^{10,59,61}$ and mitochondrial dysfunction. ${ }^{50,122}$ These varied mechanisms may act in a synchronistic manner in HD, or these toxic pathways may occur sequentially with one mechanism eliciting the next. Additionally, these different toxic mechanisms may be associated with distinct aggregate species within the complex htt aggregation landscape. 
Such a scenario would account for the apparent conflicting reports trying to pinpoint specific toxic species. Here, a subpopulation of SUMOylated htt promoted $\beta$-sheet rich aggregates is introduced that appeared as oligomers and apparent larger conglomerates of these oligomers that were SDS soluble. As it has been noted that SUMOylation promotes neurodegeneration, ${ }^{72}$ this may be due to the promotion of diffuse $\beta$-sheet rich aggregates. While pointing specifically to fibrils, mutational modification of $\mathrm{N}$-terminal fragments of htt was designed to control htt aggregation into predictable species. ${ }^{34}$ That is, constructs were designed that either quickly fibrilized despite having nonpathogenic length polyQ domains or formed stable $\alpha$-helical oligomers. The constructs forming $\beta$-sheet rich fibrils were potently toxic in cell culture and drosophila models compared to $\alpha$-helical oligomers, suggesting that the underlying amyloid structure is key to some toxic mechanism. As SUMOylation promotes $\beta$-sheet rich aggregates, the underlying toxic amyloid structure may be present in a distinct aggregate morphology, accounting for the enhanced toxicity by SUMOylation of htt.

As Nt17 mediates the interaction of htt-exon1 with lipid membranes, the impact of SUMOylation on the ability of htt to bind and disrupt TBLE bilayers was determined. There is precedent of SUMOylation of amyloid-forming proteins modifying protein interactions with lipids in cellular environments. In particular, SUMO regulates the sorting of $\alpha$-syn into extracellular vesicles, ${ }^{123}$ and modification of oligodendroglial cell membranes by DHA followed by peroxide induced oxidative stress that promotes $\alpha$-syn inclusions that are immunoreactive for SUMO1. ${ }^{124}$ Many amphipathic $\alpha$-helical lipid binding domains attach via a mechanism of initially inserting into short-lived defects in lipid membranes due to lateral diffusion of lipid components. This mechanism underlies the preference of many amphipathic $\alpha$-helices to bind to curved membranes, ${ }^{125}$ and htt-exon1 displays a preference for curved membrane. ${ }^{126}$ Within Nt17, K6 and 
K9 have been confirmed as major SUMOylation sites. ${ }^{83}$ Of these two lysine residues, K9 has been suggested to play a role in the initial binding of htt to lipid membranes. ${ }^{36}$ Attachment of the bulky SUMO protein to Nt17 likely provides a steric block to inserting into defects in lipid bilayers, preventing the ability to bind membranes.

Membrane degradation of the nuclear envelope, ER, and mitochondria has been attributed to mutant htt. ${ }^{10,59,127}$ The inhibition of lipid activity by SUMOylation suggests that enhanced htt toxicity associated with SUMOylation is not due to htt-induced membrane damage of organelles. As stated previously, there are a number of toxic mechanisms associated with mutant htt. While potentially preventing lipid interaction leading to membrane damage, SUMOylation may promote other toxic mechanisms. SUMOylation affects nucleocytoplasmic transport, resulting in the majority of SUMOylated proteins residing in the nucleus. ${ }^{128}$ Although this is quite speculative, SUMOylation may promote toxic mechanisms occurring in the nucleus, e.g. transcriptional dysregulation and DNA damage. ${ }^{115,129,130}$ Unraveling the role of distinct aggregate species and the interdependencies of htt-related toxic pathways will require careful temporal analysis of these processes within HD models. Interestingly, fluorescence correlation spectroscopy performed on htt-exon1-expressing PC12 cells provided temporal analysis of aggregate formation, revealing the early formation of oligomers with tetramers being the smallest species. ${ }^{12}$ With time, a number of higher ordered aggregates were detected, culminating in sedimentable htt aggregates. Temporally correlating DNA damage, caspase 3 induction, mitochondrial dysfunction, and cell death suggested that DNA damage was the earliest pathological event. 


\section{References}

(1) MacDonald, M. E.; Ambrose, C. M.; Duyao, M. P.; Myers, R. H.; Lin, C.; Srinidhi, L.; Barnes, G.; Taylor, S. A.; James, M.; Groot, N.; MacFarlane, H.; Jenkins, B.; Anderson, M. A.; Wexler, N. S.; Gusella, J. F.; Bates, G. P.; Baxendale, S.; Hummerich, H.; Kirby, S.; North, M.; Youngman, S.; Mott, R.; Zehetner, G.; Sedlacek, Z.; Poustka, A.; Frischauf, A. M.; Lehrach, H.; Buckler, A. J.; Church, D.; Doucette-Stamm, L.; O’Donovan, M. C.; Riba-Ramirez, L.; Shah, M.; Stanton, V. P.; Strobel, S. A.; Draths, K. M.; Wales, J. L.; Dervan, P.; Housman, D. E.; Altherr, M.; Shiang, R.; Thompson, L.; Fielder, T.; Wasmuth, J. J.; Tagle, D.; Valdes, J.; Elmer, L.; Allard, M.; Castilla, L.; Swaroop, M.; Blanchard, K.; Collins, F. S.; Snell, R.; Holloway, T.; Gillespie, K.; Datson, N.; Shaw, D.; Harper, P. S. A Novel Gene Containing a Trinucleotide Repeat That Is Expanded and Unstable on Huntington's Disease Chromosomes. Cell 1993, 72 (6), 971-983.

(2) Penney, J. B.; Vonsattel, J.-P.; Macdonald, M. E.; Gusella, J. F.; Myers, R. H. CAG Repeat Number Governs the Development Rate of Pathology in Huntington's Disease. Ann. Neurol. 1997, 41 (5), 689-692.

(3) Snell, R. G.; MacMillan, J. C.; Cheadle, J. P.; Fenton, I.; Lazarou, L. P.; Davies, P.; MacDonald, M. E.; Gusella, J. F.; Harper, P. S.; Shaw, D. J. Relationship between Trinucleotide Repeat Expansion and Phenotypic Variation in Huntington's Disease. Nat. Genet. 1993, 4, 393-397.

(4) Chen, S.; Berthelier, V.; Yang, W.; Wetzel, R. Polyglutamine Aggregation Behavior in Vitro Supports a Recruitment Mechanism of Cytotoxicity. J. Mol. Biol. 2001, 311 (1), 173-182.

(5) Chen, S.; Ferrone, F. A.; Wetzel, R. Huntington's Disease Age-of-Onset Linked to Polyglutamine Aggregation Nucleation. PNAS. 2002, 99 (18), 11884-11889.

(6) Poirier, M. A.; Li, H.; Macosko, J.; Cai, S.; Amzel, M.; Ross, C. A. Huntingtin Spheroids and Protofibrils as Precursors in Polyglutamine Fibrilization. J. Biol. Chem. 2002, 277 (43), 4103241037.

(7) Scherzinger, E.; Sittler, A.; Schweiger, K.; Heiser, V.; Lurz, R.; Hasenbank, R.; Bates, G. P.; Lehrach, H.; Wanker, E. E. Self-Assembly of Polyglutamine-Containing Huntingtin Fragments into Amyloid-like Fibrils: Implications for Huntington's Disease Pathology. PNAS. 1999, 96 (8), 4604-4609.

(8) Legleiter, J.; Mitchell, E.; Lotz, G. P.; Sapp, E.; Ng, C.; DiFiglia, M.; Thompson, L. M.; Muchowski, P. J. Mutant Huntingtin Fragments Form Oligomers in a Polyglutamine LengthDependent Manner in Vitro and in Vivo. J. Biol. Chem. 2010, 285 (19), 14777-14790.

(9) Davies, S. W.; Turmaine, M.; Cozens, B. A.; DiFiglia, M.; Sharp, A. H.; Ross, C. A.; Scherzinger, E.; Wanker, E. E.; Mangiarini, L.; Bates, G. P. Formation of Neuronal Intranuclear Inclusions Underlies the Neurological Dysfunction in Mice Transgenic for the HD Mutation. Cell 1997, 90 (3), 537-548.

(10) Liu, K.-Y.; Shyu, Y.-C.; Barbaro, B. A.; Lin, Y.-T.; Chern, Y.; Thompson, L. M.; James Shen, C.K.; Marsh, J. L. Disruption of the Nuclear Membrane by Perinuclear Inclusions of Mutant Huntingtin Causes Cell-Cycle Re-Entry and Striatal Cell Death in Mouse and Cell Models of Huntington's Disease. Hum. Mol. Genet. 2015, 24 (6), 1602-1616.

(11) Bäuerlein, F. J. B.; Saha, I.; Mishra, A.; Kalemanov, M.; Martínez-Sánchez, A.; Klein, R.; Dudanova, I.; Hipp, M. S.; Hartl, F. U.; Baumeister, W.; Fernández-Busnadiego, R. In Situ Architecture and Cellular Interactions of PolyQ Inclusions. Cell. 2017, 171 (1), 179-187.e10.

(12) Sahoo, B.; Arduini, I.; Drombosky, K. W.; Kodali, R.; Sanders, L. H.; Greenamyre, J. T.; Wetzel, R. Folding Landscape of Mutant Huntingtin Exon1: Diffusible Multimers, Oligomers and Fibrils, and No Detectable Monomer. Plos One. 2016, 11 (6), 1-22.

(13) Jayaraman, M.; Mishra, R.; Kodali, R.; Thakur, A. K.; Koharudin, L. M. I.; Gronenborn, A. M.; Wetzel, R. Kinetically Competing Huntingtin Aggregation Pathways Control Amyloid Polymorphism and Properties. Biochemistry. 2012, 51 (13), 2706-2716. 
(14) Jayaraman, M.; Kodali, R.; Sahoo, B.; Thakur, A. K.; Mayasundari, A.; Mishra, R.; Peterson, C. B.; Wetzel, R. Slow Amyloid Nucleation via $\alpha$-Helix-Rich Oligomeric Intermediates in Short Polyglutamine-Containing Huntingtin Fragments. J. Mol. Biol. 2012, 415 (5), 881-899.

(15) Lotz, G. P.; Legleiter, J.; Aron, R.; Mitchell, E. J.; Huang, S. Y.; Ng, C.; Glabe, C.; Thompson, L. M.; Muchowski, P. J. Hsp70 and Hsp40 Functionally Interact with Soluble Mutant Huntingtin Oligomers in a Classic ATP-Dependent Reaction Cycle. J. Biol. Chem. 2010, 285 (49), 3818338193.

(16) Takahashi, Y.; Okamoto, Y.; Popiel, H. A.; Fujikake, N.; Toda, T.; Kinjo, M.; Nagai, Y. Detection of Polyglutamine Protein Oligomers in Cells by Fluorescence Correlation Spectroscopy. J. Biol. Chem. 2007, 282 (33), 24039-24048.

(17) Sathasivam, K.; Lane, A.; Legleiter, J.; Warley, A.; Woodman, B.; Finkbeiner, S.; Paganetti, P.; Muchowski, P. J.; Wilson, S.; Bates, G. P. Identical Oligomeric and Fibrillar Structures Captured from the Brains of R6/2 and Knock-in Mouse Models of Huntington's Disease. Hum. Mol. Genet.2009, 19 (1), 65-78.

(18) Adegbuyiro, A.; Sedighi, F.; Pilkington, A. W.; Groover, S.; Legleiter, J. Proteins Containing Expanded Polyglutamine Tracts and Neurodegenerative Disease. Biochemistry. 2017, 56 (9).

(19) Arrasate, M.; Finkbeiner, S. Protein Aggregates in Huntington's Disease. Exp. Neurol. 2012, 238 (1), 1-11.

(20) Arrasate, M.; Mitra, S.; Schweitzer, E. S.; Segal, M. R.; Finkbeiner, S. Inclusion Body Formation Reduces Levels of Mutant Huntingtin and the Risk of Neuronal Death. Nature. 2004, 431 (7010), 805-810.

(21) Miller, J.; Arrasate, M.; Brooks, E.; Libeu, C. P.; Legleiter, J.; Hatters, D.; Curtis, J.; Cheung, K.; Krishnan, P.; Mitra, S.; Widjaja, K.; Shaby, B. A.; Lotz, G. P.; Newhouse, Y.; Mitchell, E. J.; Osmand, A.; Gray, M.; Thulasiramin, V.; Saudou, F.; Segal, M.; Yang, X. W.; Masliah, E.; Thompson, L. M.; Muchowski, P. J.; Weisgraber, K. H.; Finkbeiner, S. Identifying Polyglutamine Protein Species in Situ That Best Predict Neurodegeneration. Nat. Chem. Biol. 2011, 7 (12), 925934.

(22) Saudou, F.; Finkbeiner, S.; Devys, D.; Greenberg, M. E. Huntingtin Acts in the Nucleus to Induce Apoptosis but Death Does Not Correlate with the Formation of Intranuclear Inclusions. Cell. 1998, $95(1), 55-56$.

(23) Olshina, M. A.; Angley, L. M.; Ramdzan, Y. M.; Tang, J.; Bailey, M. F.; Hill, A. F.; Hatters, D. M. Tracking Mutant Huntingtin Aggregation Kinetics in Cells Reveals Three Major Populations That Include an Invariant Oligomer Pool. J. Biol. Chem. 2010, 285 (28), 21807-21816.

(24) Sahl, S. J.; Lau, L.; Vonk, W. I. M.; Weiss, L. E.; Frydman, J.; Moerner, W. E. Delayed Emergence of Subdiffraction-Sized Mutant Huntingtin Fibrils Following Inclusion Body Formation. Q. Rev. Biophys. 2016, 49.

(25) Sahl, S. J.; Weiss, L. E.; Duim, W. C.; Frydman, J.; Moerner, W. E. Cellular Inclusion Bodies of Mutant Huntingtin Exon 1 Obscure Small Fibrillar Aggregate Species. Sci. Rep. 2012, 2 (1), 1-7.

(26) Darnell, G.; Orgel, J. P. R. O.; Pahl, R.; Meredith, S. C. Flanking Polyproline Sequences Inhibit $\beta$ Sheet Structure in Polyglutamine Segments by Inducing PPII-like Helix Structure. J. Mol. Biol. 2007, 374 (3), 688-704.

(27) Thakur, A. K.; Jayaraman, M.; Mishra, R.; Thakur, M.; Chellgren, V. M.; Byeon, I.-J. L.; Anjum, D. H.; Kodali, R.; Creamer, T. P.; Conway, J. F.; Gronenborn, A. M.; Wetzel, R. Polyglutamine Disruption of the Huntingtin Exon $1 \mathrm{~N}$ Terminus Triggers a Complex Aggregation Mechanism. Nat. Struct. Mol. Biol. 2009, 16 (4), 380-389.

(28) Michalek, M.; Salnikov, E. S.; Bechinger, B. Structure and Topology of the Huntingtin 1-17 Membrane Anchor by a Combined Solution and Solid-State Nmr Approach. Biophys. J. 2013, 105 (3), 699-710.

(29) Burke, K. A.; Kauffman, K. J.; Umbaugh, C. S.; Frey, S. L.; Legleiter, J. The Interaction of Polyglutamine Peptides with Lipid Membranes Is Regulated by Flanking Sequences Associated with Huntingtin. J. Biol. Chem. 2013, 288 (21), 14993-15005. 
(30) Mishra, R.; Jayaraman, M.; Roland, B. P.; Landrum, E.; Fullam, T.; Kodali, R.; Thakur, A. K.; Arduini, I.; Wetzel, R. Inhibiting the Nucleation of Amyloid Structure in a Huntingtin Fragment by Targeting $\alpha$-Helix-Rich Oligomeric Intermediates. J. Mol. Biol. 2012, 415 (5), 900-917.

(31) Lotz, G. P.; Legleiter, J. The Role of Amyloidogenic Protein Oligomerization in Neurodegenerative Disease. J. Mol. Med. 2013, 91 (6), 653-664.

(32) Hoffner, G.; Djian, P. Monomeric, Oligomeric and Polymeric Proteins in Huntington Disease and Other Diseases of Polyglutamine Expansion. Brain Science.s. 2014, 4 (1), 91-122.

(33) Ast, A.; Buntru, A.; Schindler, F.; Hasenkopf, R.; Schulz, A.; Brusendorf, L.; Klockmeier, K.; Grelle, G.; McMahon, B.; Niederlechner, H.; Jansen, I.; Diez, L.; Edel, J.; Boeddrich, A.; Franklin, S. A.; Baldo, B.; Schnoegl, S.; Kunz, S.; Purfürst, B.; Gaertner, A.; Kampinga, H. H.; Morton, A. J.; Petersén, Å.; Kirstein, J.; Bates, G. P.; Wanker, E. E. MHTT Seeding Activity: A Marker of Disease Progression and Neurotoxicity in Models of Huntington's Disease. Mol. Cell. 2018, 71 (5), 675-688.e6.

(34) Drombosky, K. W.; Rode, S.; Kodali, R.; Jacob, T. C.; Palladino, M. J.; Wetzel, R. Mutational Analysis Implicates the Amyloid Fibril as the Toxic Entity in Huntington's Disease. Neurobiol. Dis. 2018, 120, 126-138.

(35) Arndt, J. R.; Chaibva, M.; Legleiter, J. The Emerging Role of the First 17 Amino Acids of Huntingtin in Huntington's Disease. Biomol. Concepts. 2015, 6 (1), 33-46.

(36) Chaibva, M.; Jawahery, S.; Pilkington, A. W.; Arndt, J. R.; Sarver, O.; Valentine, S.; Matysiak, S.; Legleiter, J. Acetylation within the First 17 Residues of Huntingtin Exon 1 Alters Aggregation and Lipid Binding. Biophys. J. 2016, 111 (2), 349-362.

(37) Arndt, J. R.; Chaibva, M.; Beasley, M.; Kiani Karanji, A.; Ghassabi Kondalaji, S.; Khakinejad, M.; Sarver, O.; Legleiter, J.; Valentine, S. J. Nucleation Inhibition of Huntingtin Protein (Htt) by Polyproline PPII Helices: A Potential Interaction with the N-Terminal $\alpha$-Helical Region of Htt. Biochemistry. 2020, 59 (4), 436-449.

(38) Saudou, F.; Humbert, S. The Biology of Huntingtin. Neuron. 2016, 89 (5), 910-926.

(39) Harjes, P.; Wanker, E. E. The Hunt for Huntingtin Function: Interaction Partners Tell Many Different Stories. Trends. Biochem. Sci. 2003, 28 (8), 425-433.

(40) Kegel-Gleason, K. B. Huntingtin Interactions with Membrane Phospholipids: Strategic Targets for Therapeutic Intervention, JHD. 2013, 2 (3), 239-250.

(41) Suopanki, J.; Götz, C.; Lutsch, G.; Schiller, J.; Harjes, P.; Herrmann, A.; Wanker, E. E. Interaction of Huntingtin Fragments with Brain Membranes - Clues to Early Dysfunction in Huntington's Disease. J. Neurochem. 2006, 96 (3), 870-884.

(42) Atwal, R. S.; Xia, J.; Pinchev, D.; Taylor, J.; Epand, R. M.; Truant, R. Huntingtin Has a Membrane Association Signal That Can Modulate Huntingtin Aggregation, Nuclear Entry and Toxicity. Hum. Mol. Genet. 2007, $16(21), 2600-2615$.

(43) De Rooij, K. E.; Dorsman, J. C.; Smoor, M. A.; Den Dunnen, J. T.; Van Ommen, G.-J. B. Subcellular Localization of the Huntington's Disease Gene Product in Cell Lines by Immunofluorescence and Biochemical Subcellular Fractionation. Hum. Mol. Genet. 1996, 5 (8), 1093-1099.

(44) Trettel, F.; Rigamonti, D.; Hilditch-Maguire, P.; Wheeler, V. C.; Sharp, A. H.; Persichetti, F.; Cattaneo, E.; MacDonald, M. E. Dominant Phenotypes Produced by the HD Mutation in STHdhQ111 Striatal Cells. Hum. Mol. Genet. 2000, 9 (19), 2799-2809.

(45) Xia, J.; Lee, D. H.; Taylor, J.; Vandelft, M.; Truant, R. Huntingtin Contains a Highly Conserved Nuclear Export Signal. Hum. Mol. Genet. 2003, 12 (12), 1393-1403.

(46) Atwal, R. S.; Desmond, C. R.; Caron, N.; Maiuri, T.; Xia, J.; Sipione, S.; Truant, R. Kinase Inhibitors Modulate Huntingtin Cell Localization and Toxicity. Nat. Chem. Biol. 2011, 7 (7), 453460.

(47) Chang, D. T. W.; Rintoul, G. L.; Pandipati, S.; Reynolds, I. J. Mutant Huntingtin Aggregates Impair Mitochondrial Movement and Trafficking in Cortical Neurons. Neurobiol. Dis. 2006, 22 (2), 388-400. 
(48) Choo, Y. S.; Johnson, G. V. W.; MacDonald, M.; Detloff, P. J.; Lesort, M. Mutant Huntingtin Directly Increases Susceptibility of Mitochondria to the Calcium-Induced Permeability Transition and Cytochrome c Release. Hum. Mol. Genet. 2004, 13 (14), 1407-1420.

(49) Gu, M.; Gash, M. T.; Mann, V. M.; Javoy-Agid, F.; Cooper, J. M.; Schapira, A. H. V. Mitochondrial Defect in Huntington's Disease Caudate Nucleus. Ann. Neurol. 1996, 39 (3), 385389.

(50) Orr, A. L.; Li, S.; Wang, C.-E.; Li, H.; Wang, J.; Rong, J.; Xu, X.; Mastroberardino, P. G.; Greenamyre, J. T.; Li, X.-J. N-Terminal Mutant Huntingtin Associates with Mitochondria and Impairs Mitochondrial Trafficking. J. Neurosci. 2008, 28 (11), 2783-2792.

(51) Panov, A. V.; Gutekunst, C.-A.; Leavitt, B. R.; Hayden, M. R.; Burke, J. R.; Strittmatter, W. J.; Greenamyre, J. T. Early Mitochondrial Calcium Defects in Huntington's Disease Are a Direct Effect of Polyglutamines. Nat. Neurosci. 2002, 5 (8), 731-736.

(52) Kegel, K. B.; Sapp, E.; Yoder, J.; Cuiffo, B.; Sobin, L.; Kim, Y. J.; Qin, Z.-H.; Hayden, M. R.; Aronin, N.; Scott, D. L.; Isenberg, G.; Goldmann, W. H.; DiFiglia, M. Huntingtin Associates with Acidic Phospholipids at the Plasma Membrane. J. Biol. Chem. 2005, 280 (43), 36464-36473. Gauthier, L. R.; Charrin, B. C.; Borrell-Pagès, M.; Dompierre, J. P.; Rangone, H.; Cordelières, F. P.; De Mey, J.; MacDonald, M. E.; Leßmann, V.; Humbert, S.; Saudou, F. Huntingtin Controls Neurotrophic Support and Survival of Neurons by Enhancing BDNF Vesicular Transport along Microtubules. Cell 2004, 118 (1), 127-138.

(54) Gunawardena, S.; Her, L.-S.; Brusch, R. G.; Laymon, R. A.; Niesman, I. R.; Gordesky-Gold, B.; Sintasath, L.; Bonini, N. M.; Goldstein, L. S. B. Disruption of Axonal Transport by Loss of Huntingtin or Expression of Pathogenic PolyQ Proteins in Drosophila. Neuron. 2003, 40 (1), 2540 .

(55) Lee, W.-C. M.; Yoshihara, M.; Littleton, J. T. Cytoplasmic Aggregates Trap PolyglutamineContaining Proteins and Block Axonal Transport in a Drosophila Model of Huntington's Disease. PNAS. 2004, 101 (9), 3224-3229.

(56) Nagarajan, A.; Jawahery, S.; Matysiak, S. The Effects of Flanking Sequences in the Interaction of Polyglutamine Peptides with a Membrane Bilayer. J. Phys. Chem. B. 2014, 118 (24), 6368-6379.

Zheng, Z.; Li, A.; Holmes, B. B.; Marasa, J. C.; Diamond, M. I. An N-Terminal Nuclear Export Signal Regulates Trafficking and Aggregation of Huntingtin (Htt) Protein Exon 1. J. Biol. Chem. 2013, 288 (9), 6063-6071.

Ueda, M.; Li, S.; Itoh, M.; Hayakawa-Yano, Y.; Wang, M.; Hayakawa, M.; Hasebe-Matsubara, R.; Ohta, K.; Ohta, E.; Mizuno, A.; Hida, Y.; Matsumoto, M.; Chen, H.; Nakagawa, T. Polyglutamine Expansion Disturbs the Endoplasmic Reticulum Formation, Leading to Caspase-7 Activation through Bax.Biochem. Biophys. Res. Commun. 2014, 443 (4), 1232-1238.

Ueda, M.; Li, S.; Itoh, M.; Wang, M.; Hayakawa, M.; Islam, S.; Tana; Nakagawa, K.; Chen, H.; Nakagawa, T. Expanded Polyglutamine Embedded in the Endoplasmic Reticulum Causes Membrane Distortion and Coincides with Bax Insertion. Biochem. Biophys. Res. Commun. 2016, 474 (2), 259-263.

(60) Pandey, N. K.; Isas, J. M.; Rawat, A.; Lee, R. V.; Langen, J.; Pandey, P.; Langen, R. The 17Residue-Long N Terminus in Huntingtin Controls Stepwise Aggregation in Solution and on Membranes via Different Mechanisms. J. Biol. Chem. 2018, 293 (7), 2597-2605.

(61) Burke, K. A.; Hensal, K. M.; Umbaugh, C. S.; Chaibva, M.; Legleiter, J. Huntingtin Disrupts Lipid Bilayers in a PolyQ-Length Dependent Manner. Biochim. Biophys. Acta. Biomembr. 2013, 1828 (8), 1953-1961.

(62) Levy, G. R.; Shen, K.; Gavrilov, Y.; Smith, P. E. S.; Levy, Y.; Chan, R.; Frydman, J.; Frydman, L. Huntingtin's N-Terminus Rearrangements in the Presence of Membranes: A Joint Spectroscopic and Computational Perspective. ACS. Chem. Neurosci. 2019, 10 (1), 472-481.

(63) Chaibva, M.; Gao, X.; Jain, P.; Campbell, W. A.; Frey, S. L.; Legleiter, J. Sphingomyelin and GM1 Influence Huntingtin Binding to, Disruption of, and Aggregation on Lipid Membranes. $A C S$. Omega. 2018, 3 (1), 273-285. 
(64) Gao, X.; Campbell, W. A.; Chaibva, M.; Jain, P.; Leslie, A. E.; Frey, S. L.; Legleiter, J. Cholesterol Modifies Huntingtin Binding to, Disruption of, and Aggregation on Lipid Membranes. Biochemistry. 2016, 55 (1), 92-102.

(65) Aiken, C. T.; Steffan, J. S.; Guerrero, C. M.; Khashwji, H.; Lukacsovich, T.; Simmons, D.; Purcell, J. M.; Menhaji, K.; Zhu, Y.-Z.; Green, K.; LaFerla, F.; Huang, L.; Thompson, L. M.; Marsh, J. L. Phosphorylation of Threonine 3 IMPLICATIONS FOR HUNTINGTIN AGGREGATION AND NEUROTOXICITY. J. Biol. Chem. 2009, 284 (43), 29427-29436.

(66) Pardo, A. D.; Maglione, V.; Alpaugh, M.; Horkey, M.; Atwal, R. S.; Sassone, J.; Ciammola, A.; Steffan, J. S.; Fouad, K.; Truant, R.; Sipione, S. Ganglioside GM1 Induces Phosphorylation of Mutant Huntingtin and Restores Normal Motor Behavior in Huntington Disease Mice. PNAS. 2012, 109 (9), 3528-3533.

(67) Thompson, L. M.; Aiken, C. T.; Kaltenbach, L. S.; Agrawal, N.; Illes, K.; Khoshnan, A.; MartinezVincente, M.; Arrasate, M.; O’Rourke, J. G.; Khashwji, H.; Lukacsovich, T.; Zhu, Y.-Z.; Lau, A. L.; Massey, A.; Hayden, M. R.; Zeitlin, S. O.; Finkbeiner, S.; Green, K. N.; LaFerla, F. M.; Bates, G.; Huang, L.; Patterson, P. H.; Lo, D. C.; Cuervo, A. M.; Marsh, J. L.; Steffan, J. S. IKK Phosphorylates Huntingtin and Targets It for Degradation by the Proteasome and Lysosome. $J$. Cell. Biol. 2009, 187 (7), 1083-1099.

(68) Gu, X.; Greiner, E. R.; Mishra, R.; Kodali, R.; Osmand, A.; Finkbeiner, S.; Steffan, J. S.; Thompson, L. M.; Wetzel, R.; Yang, X. W. Serines 13 and 16 Are Critical Determinants of FullLength Human Mutant Huntingtin Induced Disease Pathogenesis in HD Mice. Neuron. 2009, 64 (6), 828-840.

(69) Cong, X.; Held, J. M.; DeGiacomo, F.; Bonner, A.; Chen, J. M.; Schilling, B.; Czerwieniec, G. A.; Gibson, B. W.; Ellerby, L. M. Mass Spectrometric Identification of Novel Lysine Acetylation Sites in Huntingtin. Mol. Cell. Proteomics. 2011, 10 (10), M111.009829-M111.009829.

(70) DiGiovanni, L. F.; Mocle, A. J.; Xia, J.; Truant, R. Huntingtin N17 Domain Is a Reactive Oxygen Species Sensor Regulating Huntingtin Phosphorylation and Localization. Hum. Mol. Genet. 2016, 25 (18), 3937-3945.

(71) Kalchman, M. A.; Graham, R. K.; Xia, G.; Koide, H. B.; Hodgson, J. G.; Graham, K. C.; Goldberg, Y. P.; Gietz, R. D.; Pickart, C. M.; Hayden, M. R. Huntingtin Is Ubiquitinated and Interacts with a Specific Ubiquitin-Conjugating Enzyme. J. Biol. Chem. 1996, 271 (32), 19385-19394.

(72) Steffan, J. S.; Agrawal, N.; Pallos, J.; Rockabrand, E.; Trotman, L. C.; Slepko, N.; Illes, K.; Lukacsovich, T.; Zhu, Y.-Z.; Cattaneo, E.; Pandolfi, P. P.; Thompson, L. M.; Marsh, J. L. SUMO Modification of Huntingtin and Huntington's Disease Pathology. Science. 2004, 304 (5667), 100104.

(73) Subramaniam, S.; Sixt, K. M.; Barrow, R.; Snyder, S. H. Rhes, a Striatal Specific Protein, Mediates Mutant-Huntingtin Cytotoxicity. Science. 2009, 324 (5932), 1327-1330.

(74) Mishra, R.; Hoop, C. L.; Kodali, R.; Sahoo, B.; van der Wel, P. C. A.; Wetzel, R. Serine Phosphorylation Suppresses Huntingtin Amyloid Accumulation by Altering Protein Aggregation Properties. J. Mol. Biol. 2012, 424 (1), 1-14.

(75) Arndt, J. R.; Brown, R. J.; Burke, K. A.; Legleiter, J.; Valentine, S. J. Lysine Residues in the NTerminal Huntingtin Amphipathic $\alpha$-Helix Play a Key Role in Peptide Aggregation. J. Mass. Spectrom. 2015, 50 (1), 117-126.

(76) Chiki, A.; DeGuire, S. M.; Ruggeri, F. S.; Sanfelice, D.; Ansaloni, A.; Wang, Z.-M.; Cendrowska, U.; Burai, R.; Vieweg, S.; Pastore, A.; Dietler, G.; Lashuel, H. A. Mutant Exon1 Huntingtin Aggregation Is Regulated by T3 Phosphorylation-Induced Structural Changes and Crosstalk between T3 Phosphorylation and Acetylation at K6. Angew. Chem. Int. Ed. 2017, 56 (19), 52025207.

(77) Sampson, D. A.; Wang, M.; Matunis, M. J. The Small Ubiquitin-like Modifier-1 (SUMO-1) Consensus Sequence Mediates Ubc9 Binding and Is Essential for SUMO-1 Modification. J. Biol. Chem. 2001, 276 (24), 21664-21669. 
(78) Glickman, M. H.; Ciechanover, A. The Ubiquitin-Proteasome Proteolytic Pathway: Destruction for the Sake of Construction. Physiol. Rev. 2002, 82 (2), 373-428.

(79) Schnell, J. D.; Hicke, L. Non-Traditional Functions of Ubiquitin and Ubiquitin-Binding Proteins. J. Biol. Chem. 2003, 278 (38), 35857-35860.

(80) Gill, G. SUMO and Ubiquitin in the Nucleus: Different Functions, Similar Mechanism. Genes. Dev. 2004, 18 (17), 2046-2059.

(81) Iwata, A.; Nagashima, Y.; Matsumoto, L.; Suzuki, T.; Yamanaka, T.; Date, H.; Deoka, K.; Nukina, N.; Tsuji, S. Intranuclear Degradation of Polyglutamine Aggregates by the Ubiquitin-Proteasome System. J. Biol. Chem. 2009, 284 (15), 9796-9803.

(82) Yang, H.; Zhong, X.; Ballar, P.; Luo, S.; Shen, Y.; Rubinsztein, D. C.; Monteiro, M. J.; Fang, S. Ubiquitin Ligase Hrd1 Enhances the Degradation and Suppresses the Toxicity of PolyglutamineExpanded Huntingtin. Exp. Cell. Res 2007, 313 (3), 538-550.

(83) O’Rourke, J. G.; Gareau, J. R.; Ochaba, J.; Song, W.; Raskó, T.; Reverter, D.; Lee, J.; Monteys, A. M.; Pallos, J.; Mee, L.; Vashishtha, M.; Apostol, B. L.; Nicholson, T. P.; Illes, K.; Zhu, Y.-Z.; Dasso, M.; Bates, G. P.; Difiglia, M.; Davidson, B.; Wanker, E. E.; Marsh, J. L.; Lima, C. D.; Steffan, J. S.; Thompson, L. M. SUMO-2 and PIAS1 Modulate Insoluble Mutant Huntingtin Protein Accumulation. Cell. Rep. 2013, 4 (2), 362-375.

(84) Ochaba, J.; Monteys, A. M.; O’Rourke, J. G.; Reidling, J. C.; Steffan, J. S.; Davidson, B. L.; Thompson, L. M. PIAS1 Regulates Mutant Huntingtin Accumulation and Huntington's DiseaseAssociated Phenotypes in Vivo. Neuron. 2016, 90 (3), 507-520.

(85) Wacker, J. L.; Zareie, M. H.; Fong, H.; Sarikaya, M.; Muchowski, P. J. Hsp70 and Hsp40 Attenuate Formation of Spherical and Annular Polyglutamine Oligomers by Partitioning Monomer. Nat. Struct. Mol. Biol. 2004, 11 (12), 1215-1222.

(86) Whitmore, L.; Wallace, B. A. Protein Secondary Structure Analyses from Circular Dichroism Spectroscopy: Methods and Reference Databases. Biopolymers. 2008, 89 (5), 392-400.

(87) Burke, K. A.; Godbey, J.; Legleiter, J. Assessing Mutant Huntingtin Fragment and Polyglutamine Aggregation by Atomic Force Microscopy. Methods, 2011, 53 (3), 275-284.

(88) Zheng, F.; Wu, Z.; Chen, Y. A Quantitative Method for the Measurement of Membrane Affinity by Polydiacetylene-Based Colorimetric Assay. Anal. Biochem. 2012, 420 (2), 171-176

(89) Alontaga, A. Y.; Bobkova, E.; Chen, Y. Biochemical Analysis of Protein SUMOylation. Curr. Protoc. Mol. Biol. 2012, 99 (1), 10.29.1-10.29.29.

(90) LeVine, H. Thioflavine T Interaction with Amyloid $\beta$-Sheet Structures. Amyloid 1995, 2 (1), 1-6.

(91) Pedersen, J. S.; Dikov, D.; Flink, J. L.; Hjuler, H. A.; Christiansen, G.; Otzen, D. E. The Changing Face of Glucagon Fibrillation: Structural Polymorphism and Conformational Imprinting. J. Mol. Biol. 2006, 355 (3), 501-523.

(92) Groenning, M.; Olsen, L.; van de Weert, M.; Flink, J. M.; Frokjaer, S.; Jørgensen, F. S. Study on the Binding of Thioflavin T to $\beta$-Sheet-Rich and Non- $\beta$-Sheet Cavities. J. Struct. Biol. 2007, 158 (3), 358-369.

(93) Sidhu, A.; Vaneyck, J.; Blum, C.; Segers-Nolten, I.; Subramaniam, V. Polymorph-Specific Distribution of Binding Sites Determines Thioflavin-T Fluorescence Intensity in $\alpha$-Synuclein Fibrils. Amyloid. 2018, 25 (3), 189-196.

(94) Legleiter, J.; Lotz, G. P.; Miller, J.; Ko, J.; Ng, C.; Williams, G. L.; Finkbeiner, S.; Patterson, P. H.; Muchowski, P. J. Monoclonal Antibodies Recognize Distinct Conformational Epitopes Formed by Polyglutamine in a Mutant Huntingtin Fragment. The J. Biol. Chem. 2009, 284 (32), 2164721658.

(95) Wei, M.; Liu, J.; Xia, Y.; Feng, F.; Liu, W.; Zheng, F. A Polydiacetylene-Based Fluorescence Assay for the Measurement of Lipid Membrane Affinity. RSC. Advances. 2015, 5 (81), 6642066425.

(96) Legleiter, J.; Fryer, J. D.; Holtzman, D. M.; Kowalewski, T. The Modulating Effect of Mechanical Changes in Lipid Bilayers Caused by ApoE-Containing Lipoproteins on A $\beta$ Induced Membrane Disruption. ACS. Chem. Neurosci. 2011, 2 (10), 588-599. 
(97) Yates, E. A.; Owens, S. L.; Lynch, M. F.; Cucco, E. M.; Umbaugh, C. S.; Legleiter, J. Specific Domains of A $\beta$ Facilitate Aggregation on and Association with Lipid Bilayers. J. Mol. Biol. 2013, 425 (11), 1915-1933.

(98) Pifer, P. M.; Yates, E. A.; Legleiter, J. Point Mutations in A $\beta$ Result in the Formation of Distinct Polymorphic Aggregates in the Presence of Lipid Bilayers. PLoS ONE. 2011, 6 (1), e16248.

(99) Yates, E. A.; Legleiter, J. Preparation Protocols of A $\beta$ (1-40) Promote the Formation of Polymorphic Aggregates and Altered Interactions with Lipid Bilayers. Biochemistry. 2014, 53 (45), 7038-7050.

(100) Kar, K.; Jayaraman, M.; Sahoo, B.; Kodali, R.; Wetzel, R. Critical Nucleus Size for DiseaseRelated Polyglutamine Aggregation Is Repeat-Length Dependent. Nat. Struct. Mol. Biol. 2011, 18 (3), 328-336.

(101) Sivanandam, V. N.; Jayaraman, M.; Hoop, C. L.; Kodali, R.; Wetzel, R.; van der Wel, P. C. A. The Aggregation-Enhancing Huntingtin N-Terminus Is Helical in Amyloid Fibrils. J. Am. Chem. Soc. 2011, 133 (12), 4558-4566.

(102) Anderson, D. B.; Zanella, C. A.; Henley, J. M.; Cimarosti, H. Sumoylation: Implications for Neurodegenerative Diseases. In SUMO Regulation of Cellular Processes; Wilson, V. G., Ed.; Advances in Experimental Medicine and Biology; Springer International Publishing: Cham, 2017; pp 261-281.

(103) Dorval, V.; Fraser, P. E. SUMO on the Road to Neurodegeneration. Biochim. Biophys. Acta. Mol. Cell. Res. 2007, 1773 (6), 694-706.

(104) Feligioni, M.; Marcelli, S.; Knock, E.; Nadeem, U.; Arancio, O.; Fraser, P. E. SUMO Modulation of Protein Aggregation and Degradation. AIMS. Mol. Sci. 2015, 2 (4), 382.

(105) Krumova, P.; Meulmeester, E.; Garrido, M.; Tirard, M.; Hsiao, H.-H.; Bossis, G.; Urlaub, H.; Zweckstetter, M.; Kügler, S.; Melchior, F.; Bähr, M.; Weishaupt, J. H. Sumoylation Inhibits $\alpha-$ Synuclein Aggregation and Toxicity. J. Cell. Biol. 2011, 194 (1), 49-60.

(106) Kim, Y. M.; Jang, W. H.; Quezado, M. M.; Oh, Y.; Chung, K. C.; Junn, E.; Mouradian, M. M. Proteasome Inhibition Induces $\alpha$-Synuclein SUMOylation and Aggregate Formation. Journal of the Neurological Science.s. 2011, 307 (1), 157-161.

(107) Fei, E.; Jia, N.; Yan, M.; Ying, Z.; Sun, Q.; Wang, H.; Zhang, T.; Ma, X.; Ding, H.; Yao, X.; Shi, Y.; Wang, G. SUMO-1 Modification Increases Human SOD1 Stability and Aggregation.Biochem. Biophys. Res. Commun. 2006, 347 (2), 406-412.

(108) Ueda, H.; Goto, J.; Hashida, H.; Lin, X.; Oyanagi, K.; Kawano, H.; Zoghbi, H. Y.; Kanazawa, I.; Okazawa, H. Enhanced SUMOylation in Polyglutamine Diseases.Biochem. Biophys. Res. Commun. 2002, 293 (1), 307-313.

(109) Ryu, J.; Cho, S.; Park, B. C.; Lee, D. H. Oxidative Stress-Enhanced SUMOylation and Aggregation of Ataxin-1: Implication of JNK Pathway.Biochem. Biophys. Res. Commun. 2010, 393 (2), 280285.

(110) Janer, A.; Werner, A.; Takahashi-Fujigasaki, J.; Daret, A.; Fujigasaki, H.; Takada, K.; Duyckaerts, C.; Brice, A.; Dejean, A.; Sittler, A. SUMOylation Attenuates the Aggregation Propensity and Cellular Toxicity of the Polyglutamine Expanded Ataxin-7. Hum. Mol. Genet. 2010, 19 (1), 181195.

(111) Almeida, B.; Abreu, I. A.; Matos, C. A.; Fraga, J. S.; Fernandes, S.; Macedo, M. G.; GutiérrezGallego, R.; Pereira, P. J. B.; Carvalho, A. L.; Macedo-Ribeiro, S. SUMOylation of the BrainPredominant Ataxin-3 Isoform Modulates Its Interaction with P97. Biochimica et Biophysica Acta (BBA) - Molecular Basis of Disease 2015, 1852 (9), 1950-1959.

(112) Zhang, Y.-Q.; Sarge, K. D. Sumoylation of Amyloid Precursor Protein Negatively Regulates A $\beta$ Aggregate Levels.Biochem. Biophys. Res. Commun. 2008, 374 (4), 673-678.

(113) Luo, H.-B.; Xia, Y.-Y.; Shu, X.-J.; Liu, Z.-C.; Feng, Y.; Liu, X.-H.; Yu, G.; Yin, G.; Xiong, Y.-S.; Zeng, K.; Jiang, J.; Ye, K.; Wang, X.-C.; Wang, J.-Z. SUMOylation at K340 Inhibits Tau Degradation through Deregulating Its Phosphorylation and Ubiquitination. PNAS. 2014, 111 (46), $16586-16591$. 
(114) Illuzzi, J.; Yerkes, S.; Parekh-Olmedo, H.; Kmiec, E. B. DNA Breakage and Induction of DNA Damage Response Proteins Precede the Appearance of Visible Mutant Huntingtin Aggregates. $J$. Neurosci. Res. 2009, 87 (3), 733-747.

(115) Chen-Plotkin, A. S.; Sadri-Vakili, G.; Yohrling, G. J.; Braveman, M. W.; Benn, C. L.; Glajch, K. E.; DiRocco, D. P.; Farrell, L. A.; Krainc, D.; Gines, S.; MacDonald, M. E.; Cha, J.-H. J. Decreased Association of the Transcription Factor Sp1 with Genes Downregulated in Huntington's Disease. Neurobiol. Dis.2006, 22 (2), 233-241.

(116) Benn, C. L.; Sun, T.; Sadri-Vakili, G.; McFarland, K. N.; DiRocco, D. P.; Yohrling, G. J.; Clark, T. W.; Bouzou, B.; Cha, J.-H. J. Huntingtin Modulates Transcription, Occupies Gene Promoters In Vivo, and Binds Directly to DNA in a Polyglutamine-Dependent Manner. J. Neurosci. 2008, 28 (42), 10720-10733.

(117) Bolognesi, B.; Kumita, J. R.; Barros, T. P.; Esbjorner, E. K.; Luheshi, L. M.; Crowther, D. C.; Wilson, M. R.; Dobson, C. M.; Favrin, G.; Yerbury, J. J. ANS Binding Reveals Common Features of Cytotoxic Amyloid Species. ACS. Chem. Biol. 2010, 5 (8), 735-740.

(118) Nucifora, F. C.; Sasaki, M.; Peters, M. F.; Huang, H.; Cooper, J. K.; Yamada, M.; Takahashi, H.; Tsuji, S.; Troncoso, J.; Dawson, V. L.; Dawson, T. M.; Ross, C. A. Interference by Huntingtin and Atrophin-1 with CBP-Mediated Transcription Leading to Cellular Toxicity. Science. 2001, 291 (5512), 2423-2428.

(119) Balch, W. E.; Morimoto, R. I.; Dillin, A.; Kelly, J. W. Adapting Proteostasis for Disease Intervention. Science. 2008, 319 (5865), 916-919.

(120) Bence, N. F.; Sampat, R. M.; Kopito, R. R. Impairment of the Ubiquitin-Proteasome System by Protein Aggregation. Science. 2001, 292 (5521), 1552-1555.

(121) Gidalevitz, T.; Ben-Zvi, A.; Ho, K. H.; Brignull, H. R.; Morimoto, R. I. Progressive Disruption of Cellular Protein Folding in Models of Polyglutamine Diseases. Science. 2006, 311 (5766), 14711474.

(122) Costa, V.; Giacomello, M.; Hudec, R.; Lopreiato, R.; Ermak, G.; Lim, D.; Malorni, W.; Davies, K. J. A.; Carafoli, E.; Scorrano, L. Mitochondrial Fission and Cristae Disruption Increase the Response of Cell Models of Huntington's Disease to Apoptotic Stimuli. EMBO Mol. Med. 2010, 2 (12), 490-503.

(123) Kunadt, M.; Eckermann, K.; Stuendl, A.; Gong, J.; Russo, B.; Strauss, K.; Rai, S.; Kügler, S.; Falomir Lockhart, L.; Schwalbe, M.; Krumova, P.; Oliveira, L. M. A.; Bähr, M.; Möbius, W.; Levin, J.; Giese, A.; Kruse, N.; Mollenhauer, B.; Geiss-Friedlander, R.; Ludolph, A. C.; Freischmidt, A.; Feiler, M. S.; Danzer, K. M.; Zweckstetter, M.; Jovin, T. M.; Simons, M.; Weishaupt, J. H.; Schneider, A. Extracellular Vesicle Sorting of $\alpha$-Synuclein Is Regulated by Sumoylation. Acta. Neuropathol. 2015, 129 (5), 695-713.

(124) Riedel, M.; Goldbaum, O.; Wille, M.; Richter-Landsberg, C. Membrane Lipid Modification by Docosahexaenoic Acid (DHA) Promotes the Formation of $\alpha$-Synuclein Inclusion Bodies Immunopositive for SUMO-1 in Oligodendroglial Cells After Oxidative Stress. J. Mol. Neurosci. 2011, 43 (3), 290-302.

(125) Drin, G.; Antonny, B. Amphipathic Helices and Membrane Curvature. FEBS. Lett. 2010, 584 (9), $1840-1847$.

(126) Chaibva, M.; Burke, K. A.; Legleiter, J. Curvature Enhances Binding and Aggregation of Huntingtin at Lipid Membranes. Biochemistry. 2014, 53 (14), 2355-2365.

(127) Jiang, Y.; Chadwick, S. R.; Lajoie, P. Endoplasmic Reticulum Stress: The Cause and Solution to Huntington's Disease, Brain. Res. J. 2016, 1648, 650-657.

(128) Pichler, A.; Melchior, F. Ubiquitin-Related Modifier SUMO1 and Nucleocytoplasmic Transport. Traffic 2002, 3 (6), 381-387.

(129) La Spada, A. R.; Taylor, J. P. Repeat Expansion Disease: Progress and Puzzles in Disease Pathogenesis. Nat. Rev. Genet. 2010, 11 (4), 247-258.

(130) Spada, A. R. L.; Taylor, J. P. Polyglutamines Placed into Context. Neuron. 2003, 38 (5), 681-684. 


\section{Oligomerization enhances huntingtin membrane activity but is suppressed by covalent crosslinking}

\subsection{Abstract}

Huntingtin disease (HD) is a neurodegenerative disease caused by an expansion of a polyglutamine (polyQ) tract within the huntingtin (htt) protein. PolyQ expansion induces the self-assembly of htt into a variety of aggregate species ranging from small oligomers to large fibrils that eventually incorporate into large inclusion bodies. There is no general consensus on which of these aggregate states are responsible for toxicity of mutant htt. The first 17 amino acids of htt (Nt17), which directly precede the polyQ tract, play a role in initiating aggregation by facilitating the formation of oligomeric intermediates that lead to fibril formation. Nt17 also functions as a lipid membrane binding domain. Here to characterize the ability of different aggregate forms of htt to bind lipid membranes, htt was pre-aggregated, and the ability of specific aggregate species was determined by a polydiacetylene lipid binding assay. The aggregation state of htt was verified by atomic force microscopy (AFM). We find that, In Vitro, oligomers are the most active lipid binding species, while the lipid interaction was suppressed by the emergence of fibrils at the later time points. The possibility of oligomer stabilizing was investigated via a lysine crosslinker, DFDNB. The large dosage of DFDNB efficiently stabilized htt oligomers, eliminating its ability to further aggregate and interact with lipid membrane. Our data thus suggest the oligomers conformational flexibility underlie the strong membrane activity. 


\subsection{Introduction}

Huntington's disease (HD), a fatal neurodegenerative disorder, is one of many CAG repeat disorders. ${ }^{1,2}$ CAG repeats that exceed a critical threshold encode mutant polyglutamine (polyQ) domains that underlie the formation of a variety proteinaceous aggregates that are the hallmark of these diseases. ${ }^{3}$ In HD, the huntingtin protein (htt) contains the expanded polyQ domain within its first exon, and the critical threshold is $\sim 35$ repeats. ${ }^{4}$ Mutant htt is deposits as intranuclear and cytoplasmic inclusion bodies composed of fibrillar htt aggregates. ${ }^{5-7}$ Beyond these inclusions, htt aggregates into a numerous nanoscale oligomeric and fibrillar species that can be diffusely distributed in cells. ${ }^{3,8-12}$ While htt aggregation into fibrils is generally regarded to occur via a nucleus-dependent growth polymerization mechanism, ${ }^{13}$ the process can result in a complex mixture of distinct aggregate species, making it difficult to gain consensus on the prominent aggregate species involved in toxicity.

Understanding the variety and mixture of htt aggregates and their physiological activity is important because these different species could be involved in an assortment of toxic mechanisms. Survival analysis of cultured neurons suggested that inclusion formation may be a protective event as maintaining a diffuse population of htt throughout the cell had a stronger correlation with death, ${ }^{14}$ and a poor correlation between inclusion body formation and toxicity is observed in cellular models of HD. ${ }^{15}$ However, inclusions have been directly implicated in cellular abnormalities associated with HD. For example, larger perinuclear inclusions of mutant htt induce cell death by direct disruption of the nuclear envelope, ${ }^{16}$ and inclusions impinge on the ER causing membrane deformation, local impairment ER organization, and alterations in ER membrane dynamics. ${ }^{17}$ Beyond the potential role of inclusion bodies, the diffuse cellular population of htt population is a heterogeneous mixture of monomers, oligomers, ${ }^{9,18,19}$ and fibrils. ${ }^{12,19,20}$ Nanoscale 
diffuse aggregates can contain up to $1000-3000$ molecules, ${ }^{8}$ and oligomeric species on the order of dimers and tetramers have been detected. ${ }^{10}$ Collectively, this underscores the vast array of potential htt aggregate species in the diffusion fraction. Evidence points to toxic gain of function of monomeric polyQ peptides, ${ }^{21}$ htt oligomers, ${ }^{19,22,23}$ and fibrils. ${ }^{24,25}$ With this in mind, it is possible that different htt aggregate species are associated with different toxic mechanisms associated with HD.

Within htt-exon1, the polyQ domain is preceded on its N-terminal side by a seventeen amino acid sequence (Nt17) and by polyproline (polyP) and proline-rich regions on its C-terminal side. Both of these flanking sequences exert influence on htt aggregation. The addition of a 10residue polyP sequence to the C-terminus of a polyQ peptide impacts the underlying conformation of polyQ and aggregation rates. ${ }^{26-29}$ In particular, Nt17 mediates the initial interaction between htt exon1 monomers by self-association to form oligomers that aid in nucleation of fibrilliziation by bringing polyQ domains in to close proximity. ${ }^{26,30,31}$ At low concentrations, Nt17 appears intrinsically disordered. ${ }^{31,32}$ Upon interaction with a binding partner, Nt17 can adopt an amphipathic $\alpha$-helical structure that facilitates oligomer formation and is observed in many htt aggregates to some degree. ${ }^{31,33,34}$ The ability of a peptide sequence to form an amphipathic $\alpha$ helices is often associated with binding lipid membranes with high curvature in a regulated manner. ${ }^{35} \mathrm{Nt} 17$ functions as a lipid binding domain. ${ }^{32,36-40}$ These htt/lipid interactions can further modify aggregation, promoting distinct aggregation pathways in a membrane composition dependent manner. ${ }^{41-44}$ The interaction of htt with lipid membranes appears to also be polyQ length dependent. ${ }^{45,46}$

Htt localizes and associates with a variety with membranous surfaces and organelles, including mitochondria, the endoplasmic reticulum (ER), the nuclear envelope, tubulovesicles, 
endosomes, lysosomes, presynaptic and clathrin-coated vesicles, and the dendritic plasma membrane. ${ }^{6,16,47-51}$ These htt/lipid interactions often result in membrane abnormalities and disruption. ${ }^{16,51-53}$ In mouse models, brain lipids accumulate in htt aggregates and inclusions. ${ }^{54-56}$ While these observations support a role for htt/lipid interactions in modifying the aggregaton process and ultimately HD pathogenesis, little is known concerning how htt aggregation state modifies the membrane activity of htt. Here, the impact of pre-aggregation of htt-exonl on its ability to directly interact with lipid vesicles was determined. These analysis support the notion that oligomerization ehances the direct interaction between htt and lipid membranes and that fibrillization suppresses membrane activity. Within Nt17, there are three lysine residues that influence oligomerization and lipid binding. ${ }^{57-59}$ As a result, these lysine residues were targeted by 1,5-difluoro-2,4-dinitrobenzene (DFDNB) in an attempt to stabilize htt oligomers. While sufficiently large doses of DFDNB stabilized htt oligomers, this effectively negated their ability to interact with lipid vesicles, suggesting that oligomer flexibility may underscore their membrane activity.

\subsection{Materials and Methods}

\subsubsection{Expression and Purification of GST-htt-exon1 Fusion Protein}

Glutathione S-transferase (GST)-htt exon1 fusion proteins with pathogenic-length polyglutamine repeats (46Q) were expressed and purified as previously described. ${ }^{60}$ Briefly, the protein was expressed in Escherichia coli for $4 \mathrm{~h}$ at $37{ }^{\circ} \mathrm{C}$ and lysed. Proteins were purified using a BioRad low pressure liquid chromatograph equipped with a GST-affinity column. The purity and the relevant fractions were verified by SDS-PAGE. Relevant fractions were pooled and placed in dialysis at $4{ }^{\circ} \mathrm{C}$ for 2 days. To remove potential pre-aggregated species, the protein solution was centrifuged at $(22,000 \times \mathrm{g}$ for $30 \mathrm{~min})$ before any experiments. Protein concentration was 
determined by a Bradford assay. To initiate aggregation, the desired volume of protein solution was incubated with Factor Xa (Promega, Madison, WI). All experiments were carried out in a tris buffer (150 mM NaCl, Tris-HCl, pH 7.4).

\subsubsection{Atomic Force Microscopy (AFM)}

Ex situ atomic force microscopy (AFM) was used to characterize the morphology of htt aggregates. For pre-aggregation experiments, htt-exon1(46Q) $(10 \mu \mathrm{M})$ were incubated $30{ }^{\circ} \mathrm{C}$ for and sampled after $1,3,5$, and $8 \mathrm{~h}$ by taking a $3 \mu \mathrm{L}$ aliquot and depositing it on freshly cleaved mica for $1 \mathrm{~min}$. Then, the mica was rinsed with $150 \mu \mathrm{L}$ of ultrapure water and dried with a gentle stream of air. For morphological analysis of crosslinked htt, aliquots were takend before crosslinking, immediately after crosslinking, and $24 \mathrm{~h}$ after crosslinking. Samples were imaged using a Nanoscope V Multi-Mode scanning probe microscope (VEECO) equipped with a closedloop vertical engage J-scanner. Silicon-oxide cantilevers with nominal spring constant of $40 \mathrm{~N} / \mathrm{m}$ and a resonance frequency of $300 \mathrm{kHz}$ were used. Scan rates were set to $1.99 \mathrm{~Hz}$ with cantilever drive frequencies at $10 \%$ off resonance. For in situ AFM experiments that tracked the interaction of htt with supported total brain lipid extract bilayers (TBLE), a tapping mode fluid cell equipped with an $\mathrm{O}$ ring and a cantilever with nominal spring constant of $0.1 \mathrm{~N} / \mathrm{m}$ was used. The TBLE bilayer was prepared by reconstituting a lyophilized lipid film in the Tris buffer to a concentration of $1 \mathrm{mg} / \mathrm{mL}$. The lipid solution then underwent 10 freeze-thaw cycles using liquid nitrogen followed by bath sonication for 10 minutes. The resulting lipid vesicle solution was injected into the fluid cell $(30 \mu \mathrm{L})$, and the formation of the lipid bilayer was monitored by continuous AFM imaging ( $2 \mathrm{~h})$ until a complete $40 \mu \mathrm{m}$ x $40 \mu \mathrm{m}$ supported bilayer was formed. The bilayers were exposed to preformed oligomers, fibrils, or crosslinked oligomers by direct injection ( $20 \mu \mathrm{M}$ of 30 $\mu \mathrm{L}$, resulting in a final concentration of $10 \mu \mathrm{M} h t t)$ into the fluid cell. Oligomers and fibrils were 
prepared by 3 and $24 \mathrm{~h}$ of pre-incubation and verified via ex situ AFM. Crosslinked oligomers were prepared as described below. Morphological changes on the lipid bilayer were monitored by continuous imaging for $3 \mathrm{~h}$. All images were analyzed using the Matlab image processing toolbox (MathWorks) as previously described. ${ }^{61}$

For in situ AFM experiments that tracked the interaction of htt with supported total brain lipid extract bilayers (TBLE), a tapping mode fluid cell equipped with an $\mathrm{O}$ ring and a cantilever with nominal spring constant of $0.1 \mathrm{~N} / \mathrm{m}$ was used. The TBLE bilayer was prepared by reconstituting a lyophilized lipid film in the Tris buffer to a concentration of $1 \mathrm{mg} / \mathrm{mL}$. The lipid solution then underwent 10 freeze-thaw cycles using liquid nitrogen followed by bath sonication for 10 minutes. The resulting lipid vesicle solution was injected into the fluid cell $(30 \mu \mathrm{L})$, and the formation of the lipid bilayer was monitored by continuous AFM imaging $(\sim 2 \mathrm{~h})$ until a complete $40 \mu \mathrm{m} \times 40 \mu \mathrm{m}$ supported bilayer was formed. The bilayers were exposed to preformed oligomers, fibrils, or crosslinked oligomers by direct injection $(20 \mu \mathrm{M}$ of $30 \mu \mathrm{L}$, resulting in a final concentration of $10 \mu \mathrm{M}$ htt) into the fluid cell. Oligomers and fibrils were prepared by 3 and $24 \mathrm{~h}$ of pre-incubation and verified via ex situ AFM. Crosslinked oligomers were prepared as described below. Morphological changes on the lipid bilayer were monitored by continuous imaging for $3 \mathrm{~h}$.

\subsubsection{Polydiacetylene Lipid Binding Assay}

A normalized polydiacetylene (PDA) lipid binding assay was utilized to measure the interaction between htt-exon1(46Q) and TBLE. Briefly, diacetylene monomers $(10,12-$ tricosadiynoic acid) and TBLE were dissolved in a 4:1 chloroform:ethanol solution and mixed at a 2:3 molar ratio. Solutions were dried under a gentle stream of nitrogen until a fully dried lipid film was formed. These films were reconstituted in hot tris buffer $(150 \mathrm{mM} \mathrm{NaCl}$, Tris- $\mathrm{HCl}, \mathrm{pH}$ $7.4,70{ }^{\circ} \mathrm{C}$ ), sonicated at $125 \mathrm{~W}$ for $10 \mathrm{~min}$, protected from light, and left at $4{ }^{\circ} \mathrm{C}$ overnight to allow 
vesicle formation. The following day, the diacetylene monomers in the lipid vesicles were polymerized by exposure to UV light $(254 \mathrm{~nm})$, resulting in a royal blue solution that undergo a colorimetric shift to red upon mechanical stress. PDA/TBLE solutions $(400 \mu \mathrm{M})$ were incubated with different preparations of htt-exon1(46Q) at $30{ }^{\circ} \mathrm{C}$, and the blue $(650 \mathrm{~nm})$ and red $(500 \mathrm{~nm})$ absorbance ( $A_{\text {blue }}$ and $A_{\text {red }}$ respectively) were recorded every 5 min for 12 to $20 \mathrm{~h}$. The negative control consisted of equal volumes of neat buffer and PDA/TBLE solution, while the internal standard involved equal volumes of $1 \mathrm{M} \mathrm{NaOH}$ and PDA/TBLE. The $\mathrm{NaOH}$ creates repulsion between headgroups via protonation, causing a saturated colorimetric shift that can be used to normalize results. ${ }^{62}$ Additional controls with corresponding DFDNB/DTT concentration for each condition were also performed. The $\% \mathrm{CR}$ was calculated for each condition using the following equation:

$$
\% C R=\left(\frac{P B_{0}-P B}{P B_{0}}\right) \times 100
$$

where $\mathrm{PB}$ is defined as $\mathrm{A}_{\text {blue }} /\left(\mathrm{A}_{\text {blue }}+\mathrm{A}_{\text {red }}\right)$ for the negative control $\left(\mathrm{PB}_{0}\right)$ and sample condition (PB).

\subsubsection{Crosslinking htt with DFDNB}

For crosslinking oligomers, htt-exon1(46Q) was incubated (after factor Xa treatment) at 40 $\mu \mathrm{m}$ for 1 and $3 \mathrm{~h}$ on ice. Next, the htt samples were treated with varying concentration ( 2.5 to 20 fold excess relative to final htt concentration of $20 \mu \mathrm{M}$ ) of DFDNB. After mixing by inversion, the crosslinking interaction ran for $10 \mathrm{~min}$ at $20^{\circ} \mathrm{C}$. After which, the reaction was quenched by equimolar concentration of dithiothreitol (DTT). 10 min after the addition of DTT, the solutions were used in PDA or ThT assays. All crosslinked htt reactions were characterized by ex situ AFM analysis. 


\subsubsection{Thioflavin-T Aggregation Assav (ThT)}

Thioflavin T fluorescence assays (ThT, Sigma-Aldrich, St. Louis, MO) were performed to monitor fibril formation. Experiments were performed in multi-well plate format (Costar clearbottom 96-well plates) using a SpetraMax M2 microplate reader (440 nm excitation, $484 \mathrm{~nm}$ emission) with readings taken every $5 \mathrm{~min}$ for $15 \mathrm{~h}$. The ThT concentration in each well was 200 $\mu \mathrm{M}$. Experiments were performed in Costar clear-bottom 96-well plates at $37^{\circ} \mathrm{C}$ and ThT. Each experiment was performed three separate times, and within each experiment individual conditions were run in triplicate. Controls included neat buffer and corresponding concentration of DFDNB/DTT for each condition used for oligomer crosslinking.

\subsubsection{Dynamic light scattering}

Dynamic light scattering (DLS) was used to measure the size distribution of the htt control and crosslinked htt after $24 \mathrm{~h}, 3$ days, and 10 days after the crosslinking reaction. DLS experiments were performed on a 90Plus/BI-MAS nanoparticle size analyzer (Brookhaven instrument). The wavelength of the laser was $635 \mathrm{~nm}$ and the scattered light was measured at $90^{\circ}$. Htt samples were diluted to a final concentration of $4 \mu \mathrm{M}$. Due to the formation of larger aggregates in the htt control (non-crosslinked) samples and crosslinked htt with $5 \mathrm{x}$ DFDNB treatment, a high speed centrifuge step $(5,000 \mathrm{~g})$ for $20 \mathrm{~min}$ was required to pellet the larger size particles and allow the suspensions to be in the size limit range of the instrument. Measurements were taken at $25^{\circ} \mathrm{C}$, and the data was analyzed using the MAS OPTION software.

\subsubsection{MTT toxicity assay}

The cytotoxicity of htt oligomers, DFDNB crosslinker and, crosslinked oligomers was tested on the Neuro-2a (N2a) cell line (ATCC® CCL-131 ${ }^{\mathrm{TM}}$ ) using Mitochondrial 3-(4,5dimethylthiazol-2-yl)-2,5-diphenyltetrazolium bromide (MTT) reduction assay. The oligomers 
were prepared with $3 \mathrm{~h}$ incubation of htt-exon1(46Q) on the ice at a concentration of $20 \mu \mathrm{M}$. In the following step, oligomers were crosslinked with 20x or 5x DFDNB treatments under the same condition prescribed before. The crosslinked reactions were quenched by the equimolar concentration of DTT after $10 \mathrm{~min}$. The final concentration of the oligomers in the crosslinked solutions were $10 \mu \mathrm{M}$. Samples of DFDNB solutions corresponding to $200 \mu \mathrm{M}$ were prepared to measure the toxic potency of the free DFDNB crosslinker. To reduce the excess free DFDNB molecules, crosslinked oligomers and DFDNB solutions were dialyzed for $24 \mathrm{~h}$ with $(300 \mu \mathrm{L}$ of sample to $10 \mathrm{~mL}$ of dialysis buffer), and the dialysis buffer was exchanged three times. On the day of experiment, fresh htt oligomers at a concentration of $10 \mu \mathrm{M}$ were prepared to be used as a control.

In the next step, stabilized oligomers, freshly non-crosslinked oligomer controls, and DFDNB vehicle controls were added to the extracellular medium of cultured N2a cells plated in triplicate in a flat-bottom 96-wells microplate. The samples were added via serial dilution resulting in doses ranging from $0.2 \mu \mathrm{M}$ to $5 \mu \mathrm{M}$ final concentration of htt in the wells. The DFNDB concertation of the vehicle control in the wells varied from $0.2-0.08 \mu \mathrm{M}$ based on equivalent volume added for the htt samples. To facilitate transferring the oligomers, cells were permeabilized with $0.1 \%$ saponin prior to the experiment, and the MTT assay was performed $72 \mathrm{~h}$ after the addition of htt. At this time, the MTT assay was performed as described in the MTT assay kit protocol (Millipore Sigma).

\subsection{Results}

\subsubsection{Htt oligomer formation correlates with lipid binding}

While the ability of htt-exon 1 to directly bind and interact with lipid membranes is well established, little is known concerning the role of aggregation state on this process. To gain an 
initial understanding of how aggregation state impacts the ability of htt to bind lipids, $10 \mu \mathrm{M}$ httexon1(46Q) samples were pre-incubated at $30{ }^{\circ} \mathrm{C}$ for variable lengths of time $(1,3,5$, and $8 \mathrm{~h}$ ), allowing aggregation to proceed to varying extents. For these different htt incubations, aliquots were then taken to analyze the population of aggregates via ex situ AFM (Fig 3.1A). Htt oligomers were observed after $1 \mathrm{~h}$ of incubation; whereas, fibrils were not observed until the $5 \mathrm{~h}$ time point.

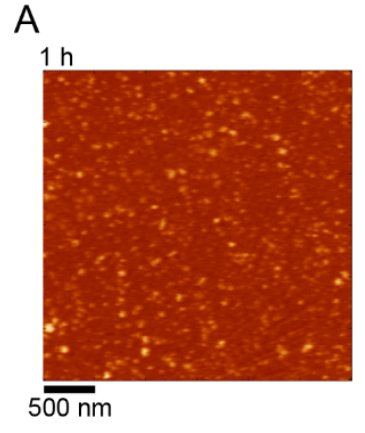

$\mathrm{B}$
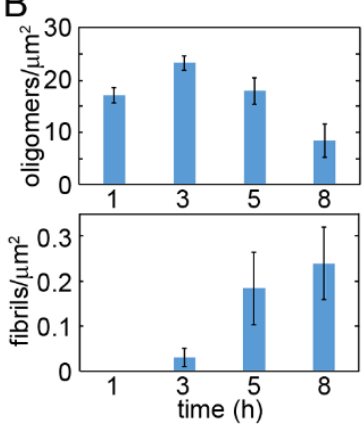

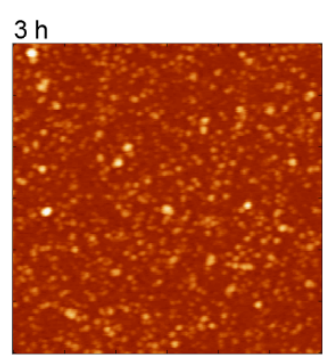

C

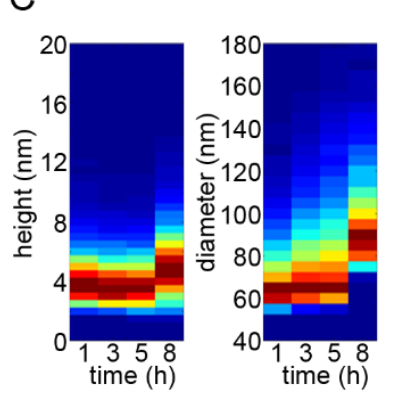

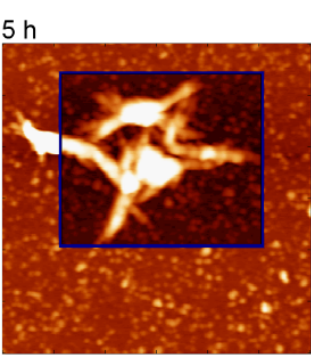
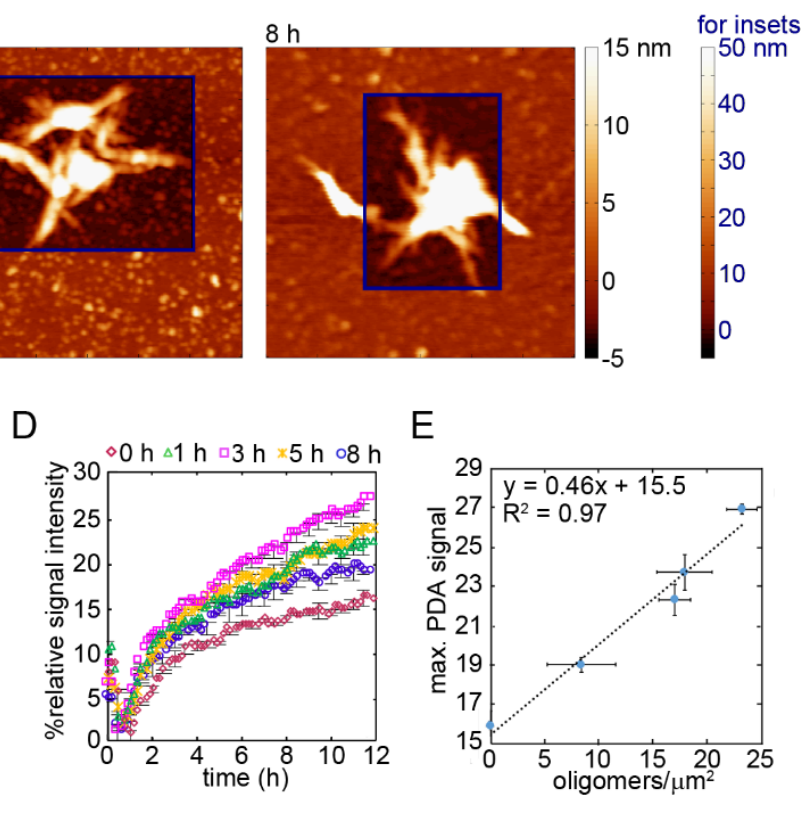

$\mathrm{E}$

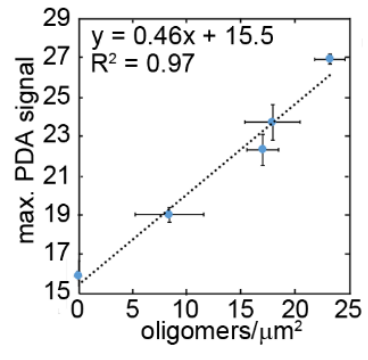

Fig 3.1 (A)Ex situ AFM images of htt-exon1(46Q) aggregates present after 1, 3, 5, or 8 h of incubation at $10 \mu \mathrm{M}$. The second color bar (labeled in blue) corresponds to the insets in the images marked by blue boxes. (B) Analysis of oligomer and fibril populations present at each time point. (C) Histograms of oligomer height and diameter as a function of time. (D) PDA/TBLE lipid binding assay performed with httexon 1(46Q) pre-incubated for various length of time. (E) The maximum PDA signal plotted as a function of the oligomers per unit area observed by AFM. Error bars represent standard deviation taken across multiple images and experiments.

To further assess the aggregate populations associated with different incubation times, automated algorithm measured morphological features of all aggregates in AFM images, and specific criteria were used to determine the number of oligomers. Oligomers were defined as particle taller than $1 \mathrm{~nm}$ with an aspect ratio of less than 2.5 , indicating a globular structure. Due to extensive bundling, the fibril population was determined by visual inspection of all images. The 
number of oligomers and fibrils per $\mu \mathrm{m}^{2}$ was determined (Fig 3.1B). A substantial number of oligomers were observed after $1 \mathrm{~h}$ of incubation $\left(17.0 \pm 1.5 \mathrm{per} \mu \mathrm{m}^{2}\right)$, and the oligomer population peaked $\left(23.2 \pm 1.4\right.$ per $\left.\mu \mathrm{m}^{2}\right)$ after $3 \mathrm{~h}$. With longer incubation times, the oligomer population steadily decreased $\left(17.9 \pm 2.5\right.$ per $\mu \mathrm{m}^{2}$ and $8.4 \pm 3.1$ per $\mu \mathrm{m}^{2}$ at 5 and $8 \mathrm{~h}$ respectively). This decrease in oligomer population occurred concurrently with a rise in the fibril population. While oligomers were present at each incubation length, there were morphological differences observed at each time points. After 1,3, and $5 \mathrm{~h}$ of incubation, oligomers had a mode height of $\sim 3.5-4.5 \mathrm{~nm}$ and a mode diameter of $\sim 60-70 \mathrm{~nm}$ (Fig 3.1C). However, a distinct tail in the diameter histogram developed with increasing incubation time. After $8 \mathrm{~h}$ of incubation, oligomers shifted to a clearly larger size (mode height of $\sim 5-6 \mathrm{~nm}$ and a mode diameter of $\sim 80-100 \mathrm{~nm}$ ).

Having characterized the htt aggregate populations associated with different incubation conditions, the extent to which the different incubations interacted with a total brain lipid extract (TBLE) bilayer was determined via a polydiacetylene (PDA) lipid binding assay (Fig 3.1E). TBLE/PDA vesicles are initially blue but mechanical stress applied to the PDA backbone by protein binding to the lipid component induces a quantifiable colorimetric response (CR). To enable comparison between unique experiments, the CR for a given batch of TBLE/PDA vesicles was normalized to a maximum $\mathrm{CR}$ determined by exposing the vesicles to a large dose of $\mathrm{NaOH} .{ }^{63}$ Addition of neat buffer was used as a negative control. These vesicles were then exposed to 10 $\mu \mathrm{M}$ preparations of htt-exon $1(46 \mathrm{Q})$ that had been incubated for various lengths of time, resulting in unique aggregate profiles. Upon exposure of PDA/TBLE vesicles to freshly cleaved httexon1(46Q) (0 h incubation, predominately monomeric), a steady increase in the PDA signal was observed, maxing out at $\sim 15.9 \%$ relative intensity after $12 \mathrm{~h}$ (Fig 3.1D). The $1 \mathrm{~h}$ incubation that contained oligomers elicited a significantly large CR, reaching $22.3 \%$ relative intensity. The $3 \mathrm{~h}$ 
incubation, which contained the largest oligomer population, invoked the largest PDA signal with a maximum value $26.9 \%$ relative intensity. The PDA signal produced by the $5 \mathrm{~h}$ incubation was less intense compared to the $3 \mathrm{~h}$ incubation, maxing out at $23.7 \%$. Interestingly, the oligomer population was comparable between the 1 and $5 \mathrm{~h}$ incubations, and the invoked PDA signals were also similar. However, the $5 \mathrm{~h}$ incubation contained a substantial fibril population as well. Lastly, the $8 \mathrm{~h}$ htt incubation, which contained fewer oligomers and more fibrils, resulted in an even greater reduction in the PDA signal (maximum of 19.0\%). The maximum PDA signal correlated linearly with the oligomer population size (Fig $3.1 \mathrm{E}, \mathrm{R}^{2}=0.97$ ), suggesting that oligomerization enhances the ability of htt to bind membranes.

A complication of the previous analysis is that these incubations still contained complex mixtures of htt monomers and aggregates. That is, as fibrils were not the predominate specie in any of the incubation, the relative fibril affinity for membranes was difficult to assess. Therefore, a TBLE/PDA assay was performed with freshly prepared (predominately monomeric) htt, oligomeric htt, and fibrillar htt at a final concentration of $10 \mu \mathrm{M}$ for all three conditions (Fig 3.2). To obtain oligomers, htt was incubated on ice for $3 \mathrm{~h}$. To obtain a predominately fibrillar sample, htt-exon 1(46Q) was seeded ( $0.25 \mu \mathrm{M}$ concentration) with preformed fibrils of a polyQ peptide and incubated for $24 \mathrm{~h}$ at $30^{\circ} \mathrm{C}$. Prior to the TBLE/PDA assay, the htt samples were checked by ex situ AFM to verify that the appropriate aggregate species were present (Fig 3.2A). The freshly prepared sampled contained a few oligomers but significantly fewer compared to the oligomeric prep. The fibril sample contained long, entangled fibrils. The oligomer prep elicited the largest CR from the TBLE/PDA assay, an $\sim 3$-fold increase in maximum signal compared with fresh htt (Fig 3.2B). Htt fibrils induced the smallest relative $\mathrm{CR}$ with a maximum signal that was significantly reduced ( $45 \%$ reduction) compared to oligomers. This further supports that oligomers have large 
membrane activity compared with either htt monomers or fibrils and that fibrils are the least membrane active species.
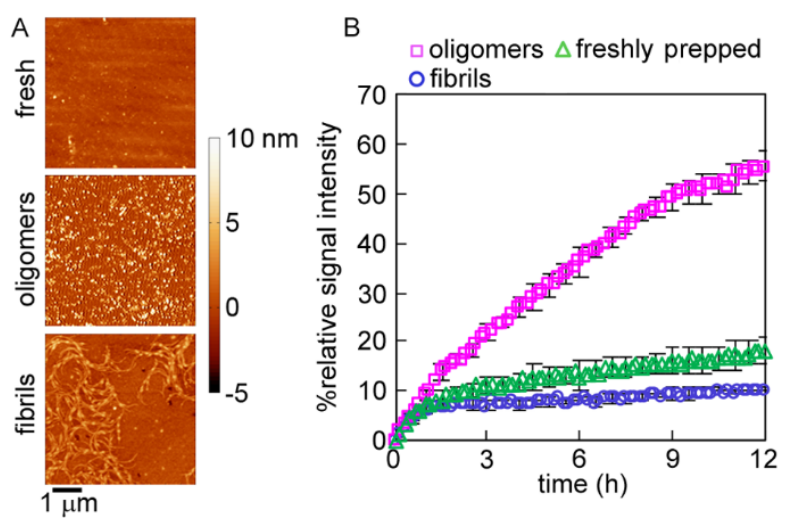

Fig 3.2 (A) Ex situ AFM images of different preparations of htt-exon1(46Q) aggregates (freshly htt, oligomers, and fibrils) that were used for (B) PDA/lipid binding assays.

\subsubsection{Crosslinking oligomers alters htt aggregation in a dose-dependent manner}

Having established that htt oligomers have a relatively large membrane activity, the possibility of stabilizing oligomers via chemical cross-linking was explored. As Nt17 promotes htt aggregation via an $\alpha$-helical oligomeric intermediate, it represents a viable target for oligomer stabilization. There are three lysine residues located within Nt17, two (K6 and K15) of which are implicated in oligomer formation. As a result, a lysine specific cross-linking agent, 1,5-difluoro2,4-dinitrobenzene (DFDNB) ${ }^{64-67}$, was employed to crosslink htt oligomers. DFDNB cross-linking is an established method of stabilizing oligomers of other amyloid-forming proteins. ${ }^{67}$ To establish conditions for oligomer stabilization, DFDNB was applied to htt incubations after 1 or $3 \mathrm{~h}$ at various doses ranging from $2.5 \times$ to $20 \times$ DFDNB to the final htt concentration of $10 \mu \mathrm{M}$. The crosslinking reaction was allowed to proceed for $10 \mathrm{~min}$ and then was quenched with DTT. To monitor the ability of the different crosslinking conditions to arrest fibril formation, Thioflavin $\mathrm{T}$ (ThT) assays were starting as soon as the DFDNB crosslinking reaction was quenched (Fig 3.3AB). Control htt-exon1(46Q) incubations that were only treated with vehicle (no DFDNB) that had 
been incubated for $1 \mathrm{~h}$ invoked a continuous increase in ThT fluorescence over $15 \mathrm{~h}$ (Fig 3.3A). Similarly, control htt-exon1(46Q) that had been pre-incubated for $3 \mathrm{~h}$ continued to invoke a ThT signal, reaching a steady-state phase at $\sim 12 \mathrm{~h}$ (Fig 3.3B). Upon covalent crosslinking after $1 \mathrm{~h}$ of pre-incubation htt with doses of $2.5 \times$ and $5 \times$ DFDNB, the rate of aggregation was actually enhanced compared to control. DFDNB treatments of $10 \times, 15 \times$, or $20 \times$ after $1 \mathrm{~h}$ of pre-incubation suppressed htt aggregation, with the $10 \times$ dose being slightly less effective. When htt was treated with different doses of DFDNB after $3 \mathrm{~h}$ of pre-incubation, only the $2.5 \times$ treatment enhanced aggregation. Doses of $5 \times$ DFDNB or higher suppressed fibrillization; although, a slight increase in ThT signal was observed for $5 \times$ and $10 \times$ towards the end of the assay.

While ThT assays suggested that larger doses of DFDNB could effectively suppress fibrillization, oligomer stabilization was further validated using ex situ AFM. This will allow for visualization of oligomeric species and comparison of their morphological features. Aliquots were taken from untreated and crosslinked htt-exon1(46Q) samples that had been pre-incubated for $3 \mathrm{~h}$ immediately after DFDNB treatment and after an additional $24 \mathrm{~h}$ of incubation for AFM analysis. In the control incubation, abundant oligomers were present at the $3 \mathrm{~h}$ time point (Fig 3.3C). After an additional $24 \mathrm{~h}$ of incubation, the control htt sample was comprised of oligomers and an extensive network of fibrils. All of the crosslinked samples appeared similar to control at the $3 \mathrm{~h}$ time point; that is, there was an extensive oligomer population.

After an additional $24 \mathrm{~h}$ of incubation, htt-exon1(46Q) that was treated with $2.5 \times$ DFDNB had formed a significant number of fibrils (Fig 3.3D), consistent with the ThT assay. These fibrils often appeared to be thicker than control fibrils with a slightly curved, less rigid appearance. Larger DFDNB treatments $(5 \times$ or higher) suppressed fibril formation and appeared to stabilize oligomers after $24 \mathrm{~h}$ of additional incubation; although, a few small protofibrils were observed with $5 \times$ and 
$10 \times$ doses of DFDNB (Fig 3.3D), which may account for the slight increase in ThT signal observed.
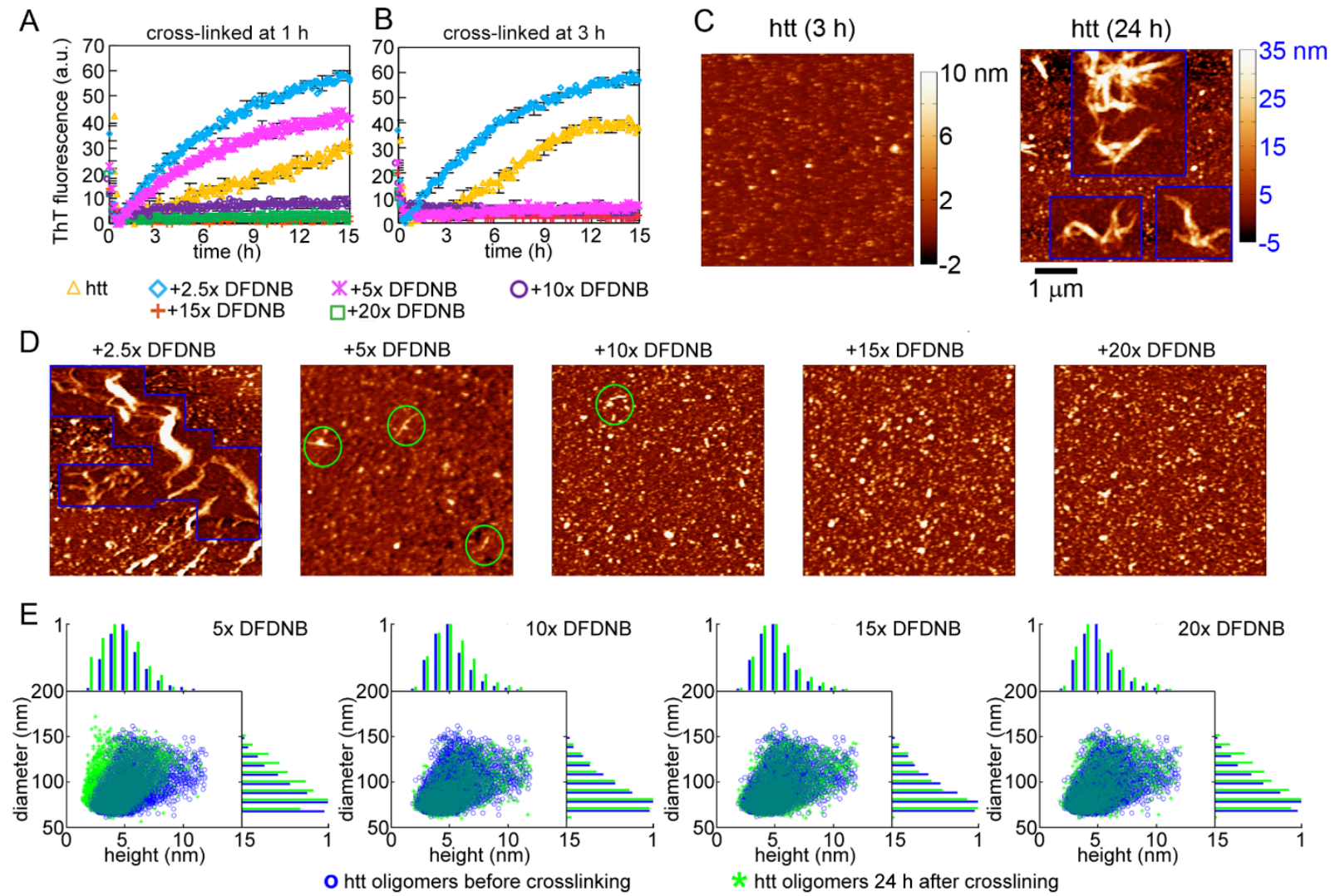

Fig 3.3 ThT assay tracking aggregation of htt crosslinked after (A) $1 \mathrm{~h}$ of pre-incubation or (B) $3 \mathrm{~h}$ of preincubation. (C) AFM images of htt without crosslinking after 3 and an additional 24 h of incubation. (D) AFM images of htt, which was crosslinked with varying doses of DFDNB after 3 h of pre-incubation, imaged after an additional $24 \mathrm{~h}$ of incubation. Green circles indicate small protofibrils. For both C and D, the second color bar (labeled in blue) corresponds to the insets in the images marked by blue boxes. (E) Correlation plots of height vs diameter of oligomers taken before crosslinking and an additional $24 \mathrm{~h}$ after crosslinking. In addition, the height (top) and diameter (right) histograms are provided.

Next, the morphological features of oligomers were measured at the $3 \mathrm{~h}$ crosslinking timepoint and compared to the morphologies after $24 \mathrm{~h}$ of additional incubation (Fig 3.3E). Comparison of diameter/height correlations and histograms indicates that the morphology of oligomers treated with high doses of DFDNB (10× or higher) were unaltered with additional incubation. However, oligomers treated with $5 \times$ DFDNB had a slight shift to larger heights. 
To further validate oligomer stabilization by DFDNB, a series of DLS experiment were performed. Oligomers were crosslinked after $3 \mathrm{~h}$ of pre-incubation with $5 \times, 10 \times, 15 \times$, or $20 \times$ DFDNB. An additional solution without crosslinking was also prepared. After 3 days of additional incubation, size distributions of each sample were measured by DLS (Fig 3.4A). The control and $5 \times$ crosslinked samples diameter distribution was not consistent with oligomers as the mode size was well over $1 \mu \mathrm{m}$ for both. In fact, these samples required a high-speed spin to pellet larger aggregates so that the remaining aggregates were within the size limits of the instrument. When crosslinked with DFDNB treatments of $10 \times$ or higher, the mode diameter of aggregates was $\sim 40$ $70 \mathrm{~nm}$, consistent with the oligomers observed by AFM. These samples treated with $10 \times$ DFDNB or higher were incubated for an additional week (10 days after initial crosslinking), and the size distributions for all three samples were remarkably similar to those measured after 3 days of additional incubation, validating the long term stability of these crosslinked oligomers (Fig 3.4BD).
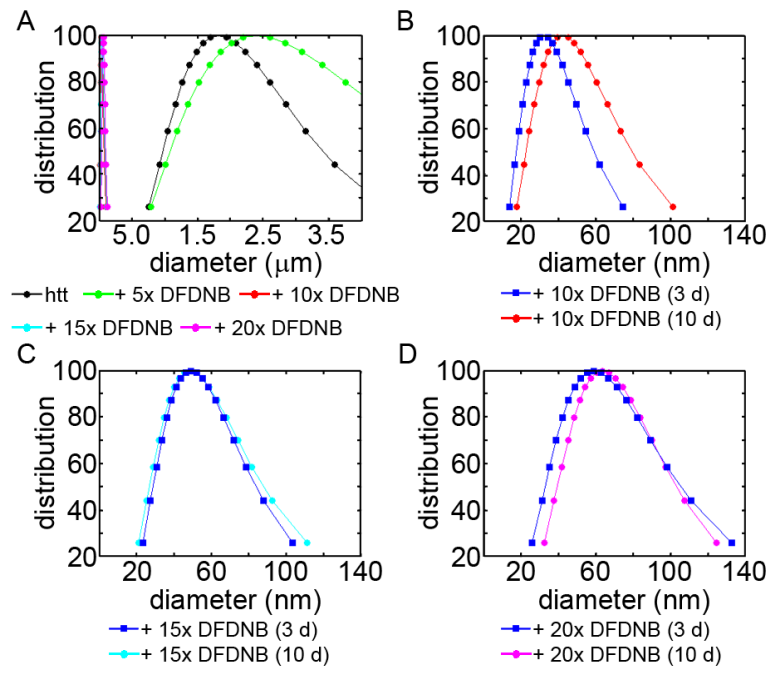

Fig 3.4 (A) Comparison of aggregate diameter measured by DLS for non-crosslinked and crosslinked htt (after 3 h of pre-incubation) with varying doses of DFDNB after an additional 3 days of incubation. Comparison of aggregate diameter of htt oligomers crosslinked after 3 h of pre-incubation with (B) 10x, (C) $15 x$, and (D) 20x DFDNB treatments after 3 and 10 days of additional incubation. 


\subsubsection{Crosslinking inhibits the ability of oligomers to bind to lipid vesicles}

Having established that htt oligomers had the highest membrane activity in comparison to monomers or fibrils, the membrane activity of DFDNB crosslinked oligomers was determined using the TBLE/PDA assay (Fig 3.5A). After $3 \mathrm{~h}$ of incubation, htt oligomers were crosslinked with DFDNB treatments ranging from $2.5 \times$ to $20 \times$. Backgrounds were accounted for in the calculation of the $\% \mathrm{CR}$, and the signal was again normalized to a maximum $\mathrm{CR}$ associated with a controlled dose of $\mathrm{NaOH}$. As a control, TBLE/PDA vesicles were exposed to untreated htt oligomers, and a large CR was observed (maximum signal of $\sim 60 \%$ ). The membrane activity of oligomers was reduced by cross-linking in a DFDNB dose dependent manner. Treatment with $2.5 \times$ DFDNB reduced the relative signal intensity associated with exposure to oligomer by $\sim 10 \%$. $5 \times$ DFDNB clearly slowed the membrane activity of oligomers, and the maximum relative signal reached after $20 \mathrm{~h}$ was cut by half compared to the untreated control. A small CR was associated with oligomers treated with $10 \times$ DFDNB, reaching a maximum relative $\mathrm{CR}$ of $\sim 12 \%$. Oligomers stabilized by $15 \times$ and $20 \times$ appeared to have virtually no membrane activity. Collectively, this suggests that stabilizing htt oligomer with DFDNB suppresses their ability to interact directly with lipid membranes (Fig 3.5A)

To further evaluate the membrane activity of oligomer (both stabilized and untreated) and fibrils, a series of in situ AFM experiments were performed to visualize morphological changes on supported TBLE bilayers upon exposure to different htt aggregate species (Fig 3.5C). The formation of a supported bilayer was achieved by direct injection of vesicles into the AFM fluid cell, and these were allowed to fuse on the mica surface with continuous monitoring by AFM imaging. Once a large $(40 \times 40 \mu \mathrm{m})$, hole-free bilayer was formed, different htt preparations were directly injected into the fluid cell to a final protein concentration of $10 \mu \mathrm{M}$. These htt preparations included 1) htt oligomers obtained by $3 \mathrm{~h}$ of pre-incubation before injection into the fluid cell, 2) 
htt oligomers crosslinked with $20 \times$ DFDNB after $3 \mathrm{~h}$ of pre-incubation, and 3) fibrils $24 \mathrm{~h}$ incubations that had been seeded. All three preparations were sampled for ex situ AFM verification of the aggregates present (Fig 3.5B).

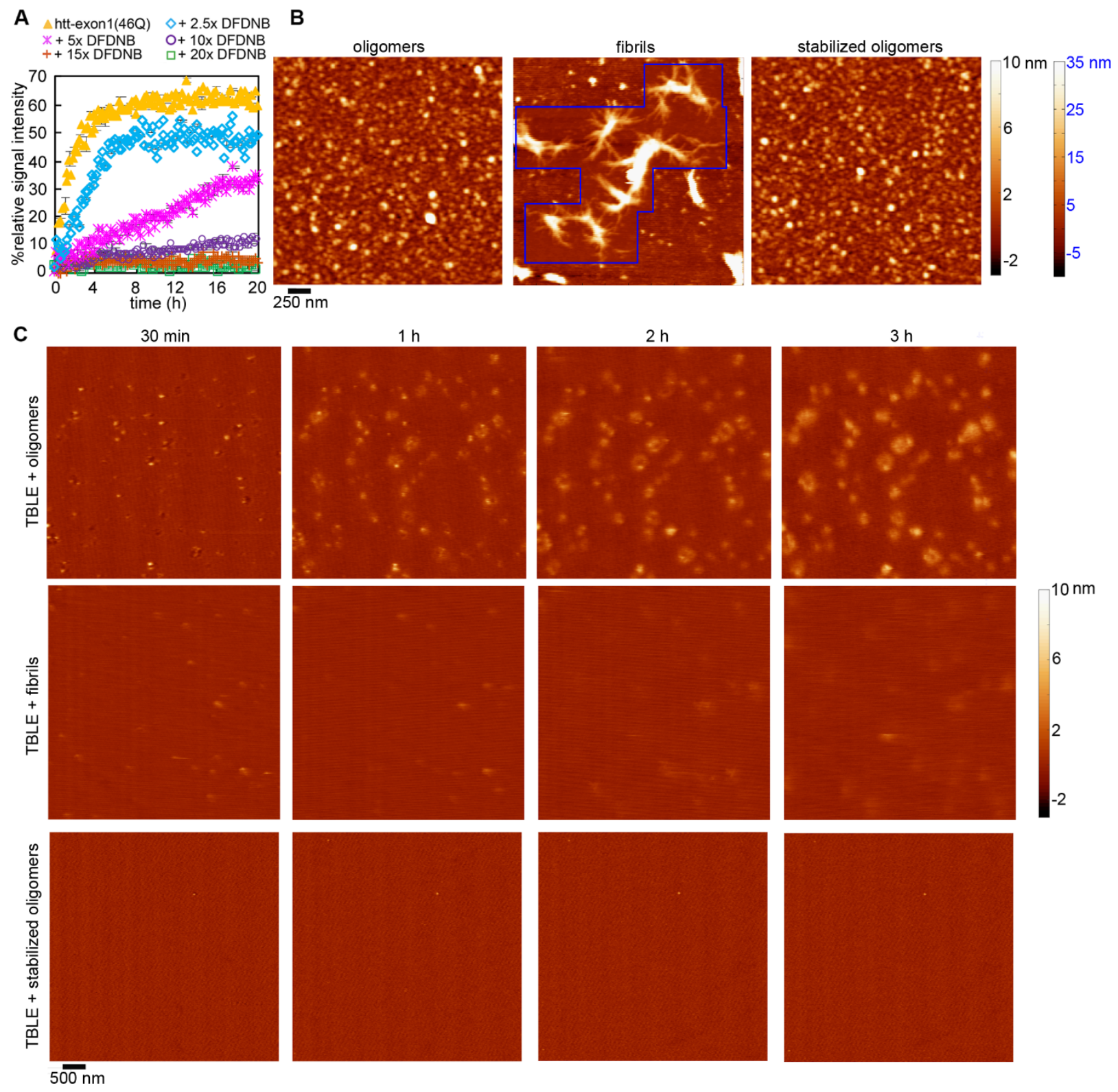

Fig 3.5 (A) The relative lipid binding affinity of htt oligomers that were pre-incbuated for $3 h$ without and with varying treatments of DFDNB crosslinking. (B) Ex situ AFM images of htt oligomers (formed after 3 $h$ of incubation), fibrils (formed by seeding and $24 \mathrm{~h}$ of incubation), and crosslinked oligomers (3 $h$ of preincubation). (C) Time course in situ AFM images of a supported TBLE bilayer exposed to htt oligomers, fibrils, and crosslinked oligomers. 
Within 30 minutes of injection, numerous htt oligomers appeared on the bilayer surface that were sometimes associated with what appeared to be small holes in the bilayer. With time, regions of increased bilayer roughness developed on the bilayer and grew. These regions were often (though not always) associated with the presence of an oligomer. In the absence of lipids, fibrils are typically observed in incubations of htt-exon 1(46Q) at this concentration in this time frame (a total of $6 \mathrm{~h}$ ). However, no fibrils were observed on the bilayer surface, consistent with reports that TBLE inhibits htt fibrillization. With pre-formed fibrils, bilayer morphology was subtly altered in some regions, and this occurred to a lesser extent in comparison to experiments with untreated oligomers. Again, no fibrils appeared on the surface, but a few oligomers were observed, which may represent residual oligomers left over in the sample prep. When exposing TBLE bilayers to stabilized htt oligomers very few (if any) crosslinked oligomers bound to the surface, and the bilayer morphology was not altered over the entire $3 \mathrm{~h}$ timeframe. The disrupted membrane regions invoked by untreated oligomers or fibrils were morphologically different. As demonstrated by height profiles (Fig 3.6A), untreated oligomers tended to invoke larger and rougher regions of disruption. The TBLE bilayer morphology observed upon exposure to DFDNB stabilized oligomers was comparable to freshly formed TBLE bilayers.

To better quantify this, the RMS roughness of the TBLE bilayer was measured as a function of time. To avoid artifacts in these measurements associated with surface coverage of these regions, two RMS roughness values were determined for samples that contained disrupted bilayer regions, one for the disrupted regions (rough) and one for the undisrupted regions (smooth). If perturbed regions were not identified, RMS values were measured across the entire image (Fig 3.6B)._A freshly formed bilayer had an RMS roughness typically smaller than $0.4 \mathrm{~nm}$ indicating a smooth bilayer. Upon exposure to untreated htt oligomers, rough regions of RMS roughness were $1.9 \pm$ 
$0.3 \mathrm{~nm}$ after $30 \mathrm{~min}$. The RMS roughness appeared to increase with time $(2.8 \pm 0.9 \mathrm{~nm}$ at $3 \mathrm{~h})$. The disrupted bilayer regions observed upon exposure to pre-formed fibrils were less pronounced with RMS roughness values falling between 0.6 and $0.9 \mathrm{~nm}$, which was still significantly larger than a fresh TBLE bilayer. For exposure to both untreated oligomers and pre-formed fibrils, the undisrupted (smooth) portions of the bilayer had RMS roughness values similar to a freshly prepared bilayer. DFDNB oligomers did not disrupt a large enough portion of the bilayer to obtain meaningful RMS values of rough areas; however, the RMS roughness measured over the entire image for all time points was indistinguishable from a fresh TBLE bilayer.

To quantify the extent of bilayer disruption by the different htt samples, pattern recognition algorithms were used to identify regions of the bilayer morphology that were disrupted or unperturbed, allowing for the percentage of the bilayer surface disrupted to be determined as a function of time (Fig 3.6C). After $30 \mathrm{~min}$ of exposure to untreated htt oligomers, $0.9 \pm 0.2 \%$ of the bilayer surface was disrupted, and the extent of disrupted bilayer steadily increased with time, reaching $10.7 \pm 1.6 \%$ within $3 \mathrm{~h}$. Fibrils disrupted the bilayer to a significantly lesser extent, with only $4 \pm 0.4 \%$ of the bilayer being altered after $3 \mathrm{~h}$. DFDNB Crosslinked oligomers did not induce extensive membrane disruption over $3 \mathrm{~h}$. The $\%$ area disrupted was less than $1 \%$ at each time point, and these regions were predominately discrete oligomers appearing on the surface rather than extensively disrupted regions of the bilayer. 
A oligomers

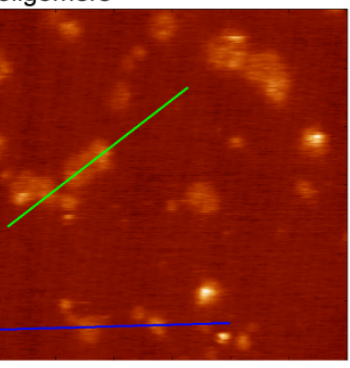

fibrils

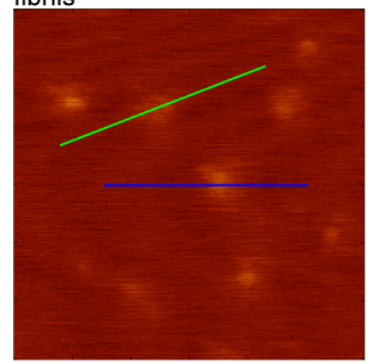

DFDNB oligomers

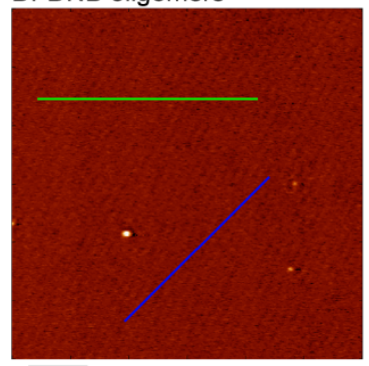

$5 \overline{00 \mathrm{~nm}}$
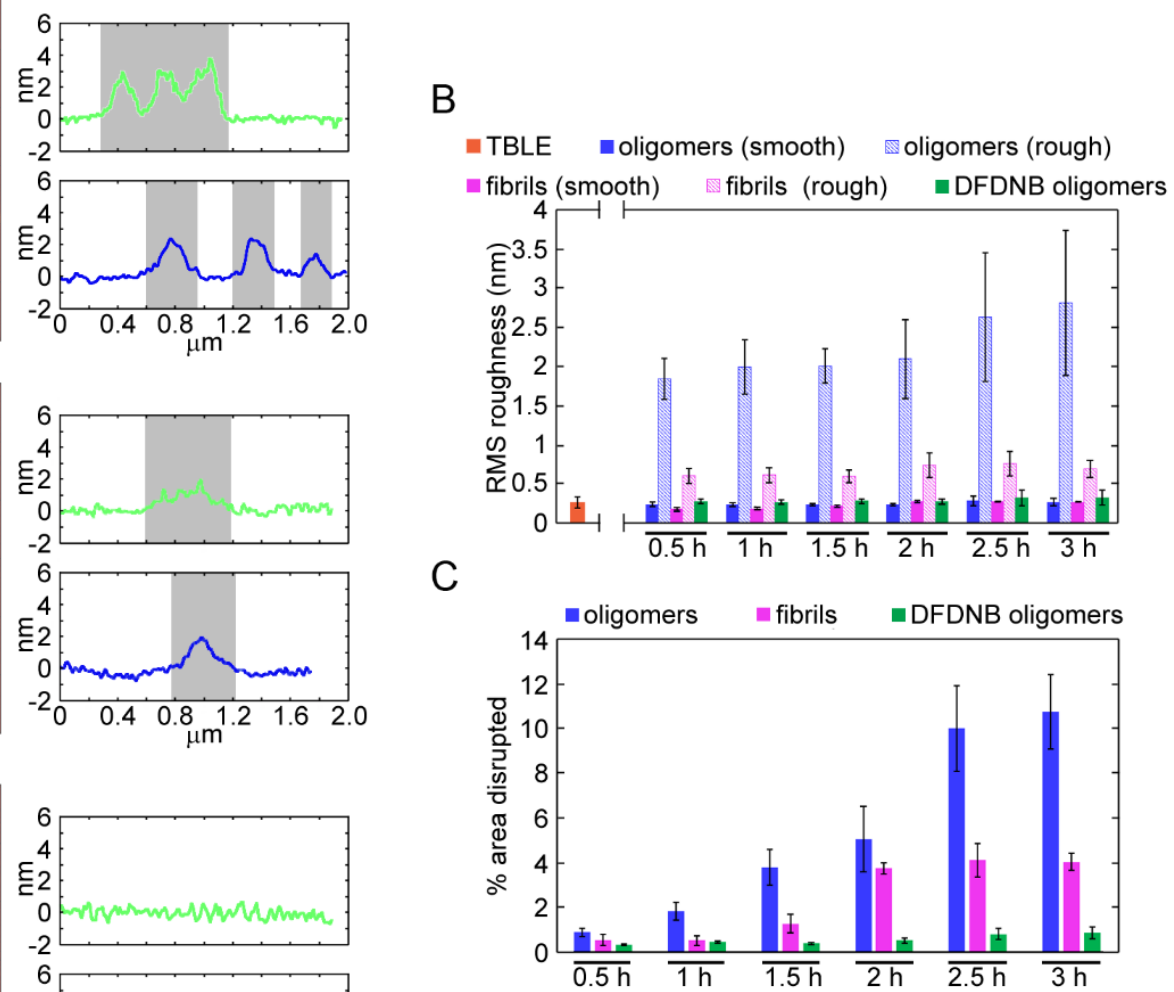

Fig 3.6 (A) Comparison of the morphology of supported TBLE bilayers exposed to oligomers, fibrils, or DFDNB stabilized oligomers. The colored lines in each in situ AFM image corresponds to the height profiles provided to the right. In each height profile, the gray shaded areas correspond to regions of the bilayer with increased surface roughness. (B) Comparison of the RMS roughness associated with a TBLE bilayer and the smooth and rough regions observed after exposure to htt oligomers, fibrils, and DFDNB stabilized oligomers as a function of time. As rough regions in the bilayer did not develop upon exposure to DFDNB stabilized oligomers, RMS roughness was measured over the entire image. (C) Comparison of the \% area of the TBLE bilayer that was disrupted by exposure to htt oligomers, fibrils, and DFDNB stabilized oligomers as a function of time. For both $B$ and $C$, the error bars represent standard deviation across three independent experiments.

\subsubsection{Crosslinking reduces oligomer toxicity}

To determine of DFDNB crosslinking altered htt oligomer toxicity, an MTT cytotoxicity assay was performed on N2a neuronal cultured cells. These cells were exposed to freshly prepared htt-exon1(46Q) oligomers, DFDNB stabilized oligomers (5x and 20x treatments, and a DFDNB vehicle control). The unmodified and crosslinked oligomers were added to the culture medium in 
doses ranging from $0.2-5 \mu \mathrm{M}$ final concentration in the wells. To aid in uptake of the htt oligomers, the cells were permeabilized with saponin. ${ }^{68,69}$ The concentration of DFDNB in vehicle controls ranges at a maximum $\sim 0.008 \mu \mathrm{M}$ to $0.2 \mu \mathrm{M}$ based on the amount needed to crosslink the htt oligomers at 20x, dilution by dialysis, and the final volume added to each well. Complicating the assay, DFDNB itself was toxic to cells. Exposure to $0.3 \mu \mathrm{M}$ or higher DFDNB reduced cell viability to less than $23 \%$ relative to control (N2a cells not exposed to htt or DFDNB); however, cell viability was $67.3 \pm 4.0 \%$ with exposure to $0.12 \mu \mathrm{M}$ DFDNB (corresponding to the $0.2 \mu \mathrm{M}$ htt concentration).

Unmodified htt-exon1(46Q) oligomers (no DFDNB) were toxic to N2A cell in a dose dependent manner (Fig 3.7). Cell viability was $15.8 \pm 1.0 \%$ relative to control with $5 \mu \mathrm{M}$ doses of unmodified htt oligomers, and this increased to $40.4 \pm 3.6 \%$ upon exposure to unmodified htt oligomer at $0.2 \mu \mathrm{M}$. With stabilized oligomers treated with $5 \mathrm{x}$ or $20 \mathrm{x}$ DFDNB, there was not a significant change in cell viability at doses greater than $1.5 \mu \mathrm{M}$; although, there is a trend of increased viability relative to control with as the concentration decreases. The issue in interpreting this result is that there is toxicity associated with untreated htt and residual DFDNB. With exposure to $0.5 \mu \mathrm{M}$ stabilized oligomers, there is a not quite statistically significant $(\mathrm{p}<0.05)$ increase in cell viability $(39.1 \pm 5.5 \%$ relative to control) with $5 x$ DFDNB stabilized oligomers, and both $5 x$ $(96.8 \pm 4.3 \%$ cell viability) and $20 \mathrm{x}(75.7 \pm 4.8 \%$ cell viability $)$ treated DFDNB oligomers are significantly less toxic (both have $\mathrm{p}<0.01$ compared to untreated htt oligomers). With regard to the 20x DFDNB treated oligomers, the cell viability mirrored closely the viability observed with the DFDNB alone control. In short, unmodified htt (no DFDNB added) considerably reduced cell viability with doses as low as $0.2 \mu \mathrm{M}$. Vehicle control containing DFDNB (but no htt) reduced cell viability as well, potentially masking the impact of DFDNB treatment on htt oligomer toxicity 
at high protein concentrations. However, as the DFDNB was effectively diluted out with smaller doses of htt, DFDNB treated oligomers became less toxic relative to htt control. A plausible explanation is that crosslinking reduces oligomer toxicity, but crosslinking also removes free DFDNB from solution, effectively reducing DFDNB toxicity as well. As a result, as residual DFDNB toxicity is removed, it becomes clearer the crosslinked htt oligomers are less toxic than their unmodified counterparts, suggesting a loss in membrane activity results in a corresponding reduction of htt-induced toxicity.

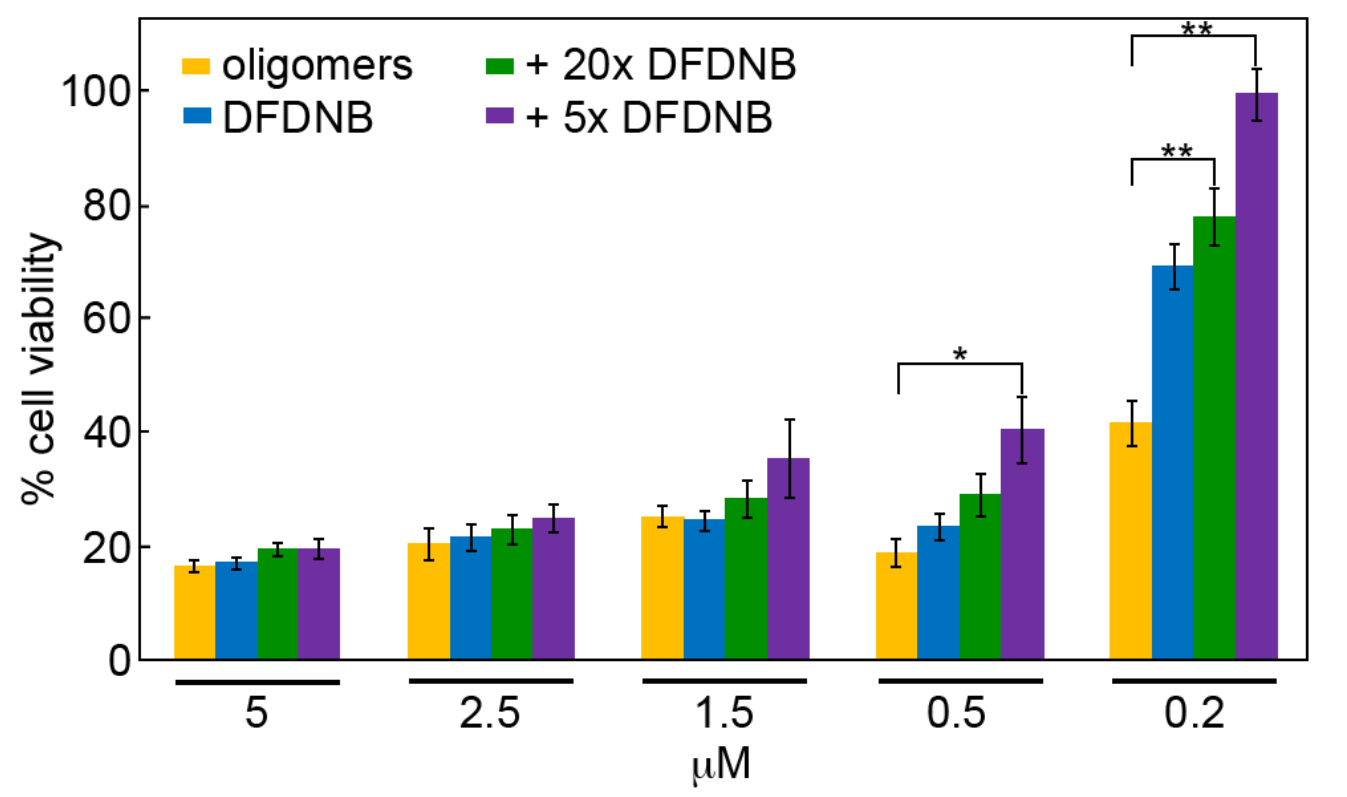

Fig 3.7 MTT toxicity assay for the N2a cells exposed to oligomers and crosslinked oligomers with $5 x$ and $20 x$ DFDNB treatment. * indicates $p<0.05$, ** indicates $p<0.01$ based on a t test $(n=3)$. Error bars represent standard error of the mean (SEM).

\subsection{Discussion}

Mutant htt is implicated in structural abnormalities and dysfunction of a variety of membranous organelles. ${ }^{6,70-73}$ While damage to a variety of subcellular membranes is evident in $\mathrm{HD}$, little is known regarding how specific aggregate species interact with lipid membranes. Here, the membrane activity of various aggregation states of htt was determined, which implicated 
oligomer of being particularly potent in binding lipids. Extensive fibril formation dramatically reduced the ability of htt to directly interact with membranes. Having established the membrane activity of htt oligomers, a crosslinking strategy was employed to stabilize these aggregate species. Due to the role of lysine residues in aggregation and oligomer structure, ${ }^{34,59}$ a known lysine specific crosslinking agent, DFDNB, was used. Rather than stabilizing oligomers, small doses of DFDNB could accelerate fibrillization, but with larger doses oligomer stabilization was achieved. However, stabilization precluded membrane activity and reduced cytotoxicity of the htt oligomers. Collectively, these results suggest some sort of conformational flexibility underlies the membrane activity of htt oligomers and that targeting structural features of htt oligomers represents a strategy to block their ability to disrupt membranes, reducing oligomer cytotoxicity.

Lipid membranes are known modulators of htt aggregation. ${ }^{41,44,74}$ However, fibril can be either stimulated ${ }^{41,48}$ or reduced ${ }^{36,42,43,46,57,74}$ by membranes depending on the precise lipid composition. Membrane activity of htt-exon1 is facilitated by Nt17, ${ }^{36,37,75}$ and mutant htt disrupts a variety of organelle membranes, leading to their dysfunction. ${ }^{16,48,51,76,77}$ Beyond this, distinct htt aggregates are linked to specific membrane abnormalities. For example, htt inclusions impinge upon the $\mathrm{ER}^{17}$ and the nuclear envelope ${ }^{16}$ leading to altered membrane dynamics, deformation, and disruption. While these studies associated htt fibrils/inclusions with changes in ER membranes or the nuclear envelope, this may represent a late stage marker of earlier events, as it is plausible that subtle alterations in these membranes are induced by earlier htt aggregates or even the aggregation process. Such a notion is supported by the ability of un-aggregated mutant htt to invoke localized morphological and mechanical changes on model lipid membranes ${ }^{36,46,78}$ and our observation reported here that fibrils have significantly reduced membrane activity. In addition, N-terminal fragments of mutant htt, such exon1 preferentially segregated to membrane fractions 
in HD brains. ${ }^{79} \mathrm{Htt}$ oligomers have been associated with inducing mitochondrial fragmentation and ER stress..$^{50,80}$

With regard to oligomers having larger membrane activity in comparison to monomeric or fibrillar htt, a plausible mechanism involves availability of induced $\alpha$-helical structure of Nt17 (Fig 3.8A). The Nt17-mediated htt membrane binding involves four basic steps: approach, reorganization, anchoring, and insertion. ${ }^{81}$ In short, an intrinsically disordered Nt17 stabilizes its interaction with lipid membranes by folding into an amphipathic $\alpha$-helix. In the time it takes for reorganizing into an $\alpha$-helix, there is a chance that $\mathrm{Nt} 17$ could dissociate from the lipid bilayer. ${ }^{57}$ Nt17's ability to form amphipathic $\alpha$-helices promotes inter-protein association into oligomeric intermediates that enhance fibril formation. ${ }^{30,31}$ Structurally, htt tetramers pack into $\alpha$-helical bundles of Nt17 in an antiparallel fashion. ${ }^{34}$ In solution, Nt17 exists in a concentration-dependent equilibrium between an intrinsically disordered monomeric state and these $\alpha$-helical oligomers. ${ }^{26,31,82}$ The stabilization of the Nt17 $\alpha$-helix by oligomerization may bypass the reorganization step, enhancing the membrane activity of oligomers. Such a mechanism requires two assumptions: 1) that preformed $\alpha$-helixes indeed bind faster and 2) that oligomer structures are flexible enough for $\mathrm{Nt17} \alpha$-helices to be available for interaction with lipids. Computational studies support the first assumption as preconfiguring Nt17 into $\alpha$-helixes speeds up the interaction between Nt17 and lipids. ${ }^{83}$ The second assumption is supported by our observation that crosslinking oligomers, and thus reducing their conformational flexibility, leads to a loss of membrane activity.

With regards to fibrils being less membrane active, this is likely due to availability of Nt17 in the fibril structure. Htt fibrils are comprised of a rigid amyloid core of polyQ domains. ${ }^{84-86}$ This core is surrounded by both the Nt17 and proline-rich domains (PRD). While there is some 
variations from different studies, the PRD appears to be more flexible and solvent exposed of the two flanking regions in the fibril structure. ${ }^{84,87-89}$ In reported fibril structures, the dynamics and conformation of Nt17 varies, ranging from being completely buried in a packed structure, ${ }^{88}$ to dynamic heterogeneity with partially dynamic residues,${ }^{87}$ or to being helical with partial immobilization by dense packing of flanking sequences on the fibril surface. ${ }^{33,84,90}$ In our study, decreased lipid binding affinity of pre-aggregated htt fibrils most likely support the notion that $\mathrm{Nt} 17$ domain is either not accessible within mature fibrils or at least is partially bracketed with non-helical structure that restrict initial binding and further insertion of htt fibrils into lipid membrane. Molecular dynamic studies suggest that the polyQ region forms hydrogen bonds with the phospholipid head groups, providing a scaffold for further insertion of Nt17 into the lipid membrane core ${ }^{81}$ The unavailability of polyQ to function in this capacity due to dense packing in the amyloid core would also reduce the membrane activity of fibrils. Collectively, the differences in membrane activity between oligomers and fibrils suggests a key role of structural flexibly in htt/lipid interactions.

While high doses of DFDNB crosslinker stabilized oligomers, it was surprising that low doses accelerated fibrillization. As both intra and intermolecular crosslinking occurs with DFDNB, such a strategy results in an inherent complexity of crosslinking products. ${ }^{91-96}$ DFDNB-modified lysine residues may initially serve as intermediates which either interact with another lysine residue intermolecularly or intramolecularly. Low concentration of DFDNB relative to the target substrate promotes intramolecular crosslinking; whereas, efficiency of intermolecular crosslinking is enhanced with higher DFDNB doses. ${ }^{94}$ With this in mind, it should be noted that with three lysine residues in Nt17 that lower doses of DFDNB resulted in an excess of target residues, which would promote intramolecular crosslinking. Based on acetylation studies, lysine 9 appears to be 
the most readily available lysine residue for modification..$^{57,97}$ As a result, it represents the most likely modified lysine residue with lower doses of DFDNB. Based on available structural analysis of Nt17 derived oligomers, lysine 9 is not involved in structural stabilizing intermolecular interactions $^{34,59,98}$ and would not be well positioned to be involved in intermolecular crosslinks. However, there is the potential to form intramolecular crosslinks between lysine 9 and lysine 6 , which would be relatively close in space within an $\alpha$-helix, with an approximate separation distance of 3-4 $\AA$ that correlates well with the DFDNB arm length of $\sim 3.5 \AA$. Lysine 9 and lysine 15 would be further spaced apart. Such an intramolecular crosslink could push the equilibrium between disordered Nt17 monomers and higher order multimers toward oligomerization without restricting conformational freedom needed for initiating fibrillization. Such a scenario may underlie the observed enhanced fibril formation with low doses of DFDNB. With larger DFDNB treatments, intermolecular crosslinks associated with lysine 6 and 9 form, stabilizing oligomers. While a clear consensus of the fate of $\mathrm{Nt17}$ in fibrils is lacking ${ }^{99}$ forming the densely packed polyQ amyloid core likely requires some conformational flexibility within the Nt17-mediated oligomer intermediate, which a high density of intermolecular crosslinks restricts. The necessity of such a structural transition represent the rate limiting step in which fluid oligomeric complexes undergo stochastic rearrangement to nucleate fibril formation in the yeast prion protein Sup $35 .{ }^{100}$

With low doses of DFDNB that promoted fibril formation, the partial reduction of membrane activity by non-stabilized oligomers may encompass a variety of mechanisms. By promoting aggregation, the highly membrane active oligomer population would be reduced as membrane inactive fibrils form. In addition, it is possible that nucleophilic substitution of fluorine atoms of DFDNB to the primary amine of lysine residues ${ }^{101}$ within $\mathrm{Nt} 17$ removes cationic character that mediates lipid binding. . $^{37,38,57,74,81}$ With higher doses of DFDNB, conformational 
flexibility of $\alpha$-helical bundles is compromised by intermolecular DFDNB-mediated crosslinking of lysine residues, and the membrane activity of stabilized oligomers is completely inhibited (Fig 3.8B). This is likely due to abundant intermolecular crosslinks that stabilized htt oligomers but suppresses the conformational flexibility required for binding lipid membranes, in the same manner by which these crosslinks prevent fibril formation.

Despite years of studies, the precise molecular mechanism of htt aggregation and its realation to cellular toxicity is not completely understood. The temporal emergence of various aggregate states has been linked to numerous pathological events associated with mutant htt. While initially inclusions were thought to be toxic species, ${ }^{5,70}$ diffusely distributed htt within the cell correlates well with toxicity. ${ }^{14,15}$ This diffusely distributed htt, however, is comprised of a heterogeneous mixture of monomers, oligomers, and fibrils. ${ }^{11,12,102-104}$

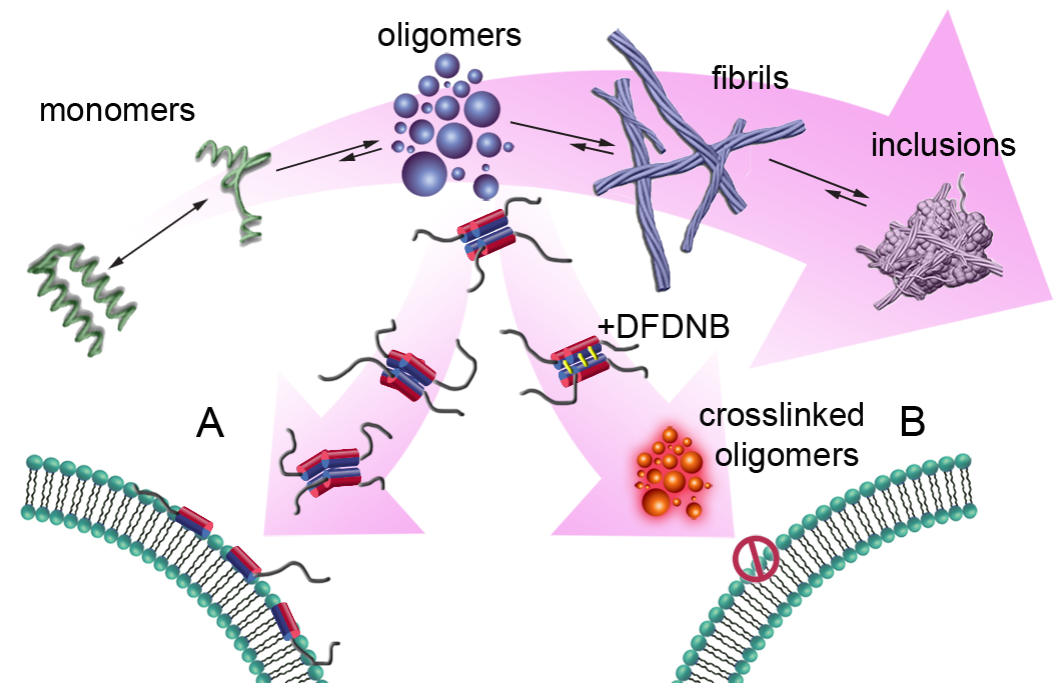

Fig 3.8 The proposed mechanism for interaction of (A) oligomers and (B) crosslinked oligomers with lipid membrane

This complexity, along with the metastability of many aggregate intermediates, has made precise assignment of toxic gain of function to specific aggregate species difficult. In addition, the transient nature of aggregate intermediates has impeded their structural characterization needed to 
fully unravel mechanistic details of toxicity. Due to these issues being common across many amyloid diseases, a variety of crosslinking methods have been employed to stabilize oligomeric intermediates so that structural and functional analysis can be performed. ${ }^{65-67,105}$ Our results have several implications for these strategies. First, crosslinking can alter the biophysical properties of oligomers, altering their exogenous interactions. Here, it was demonstrated that altering conformational flexibility impacts the membrane activity and toxicity of htt oligomers. This implies that htt membrane association plays a key role in toxicity. It could also be that the further aggregation of oligomers is required for toxicity, that is, the process of aggregation causes cellular damage rather than a discreet aggregate. Second, this impact on oligomer membrane activity suggests that crosslinking or otherwise interfering with conformational flexibility of oligomers may represent a therapeutic strategy. However, such an approach would require a crosslinking method that is more selective than DFDNB or other commonly used crosslinkers. 


\section{References}

(1) Gatchel, J. R.; Zoghbi, H. Y. Diseases of Unstable Repeat Expansion: Mechanisms and Common Principles. Nat. Rev. Genet. 2005, 6 (10), 743-755.

(2) Riley, B. E.; Orr, H. T. Polyglutamine Neurodegenerative Diseases and Regulation of Transcription: Assembling the Puzzle. Genes Dev. 2006, 20 (16), 2183-2192.

(3) Adegbuyiro, A.; Sedighi, F.; Pilkington, A. W.; Groover, S.; Legleiter, J. Proteins Containing Expanded Polyglutamine Tracts and Neurodegenerative Disease. Biochemistry. 2017, 56 (9).

(4) MacDonald, M. E.; Ambrose, C. M.; Duyao, M. P.; Myers, R. H.; Lin, C.; Srinidhi, L.; Barnes, G.; Taylor, S. A.; James, M.; Groot, N.; MacFarlane, H.; Jenkins, B.; Anderson, M. A.; Wexler, N. S.; Gusella, J. F.; Bates, G. P.; Baxendale, S.; Hummerich, H.; Kirby, S.; North, M.; Youngman, S.; Mott, R.; Zehetner, G.; Sedlacek, Z.; Poustka, A.; Frischauf, A. M.; Lehrach, H.; Buckler, A. J.; Church, D.; Doucette-Stamm, L.; O’Donovan, M. C.; Riba-Ramirez, L.; Shah, M.; Stanton, V. P.; Strobel, S. A.; Draths, K. M.; Wales, J. L.; Dervan, P.; Housman, D. E.; Altherr, M.; Shiang, R.; Thompson, L.; Fielder, T.; Wasmuth, J. J.; Tagle, D.; Valdes, J.; Elmer, L.; Allard, M.; Castilla, L.; Swaroop, M.; Blanchard, K.; Collins, F. S.; Snell, R.; Holloway, T.; Gillespie, K.; Datson, N.; Shaw, D.; Harper, P. S. A Novel Gene Containing a Trinucleotide Repeat That Is Expanded and Unstable on Huntington's Disease Chromosomes. Cell. 1993, 72 (6), 971-983.

(5) Davies, S. W.; Turmaine, M.; Cozens, B. A.; DiFiglia, M.; Sharp, A. H.; Ross, C. A.; Scherzinger, E.; Wanker, E. E.; Mangiarini, L.; Bates, G. P. Formation of Neuronal Intranuclear Inclusions Underlies the Neurological Dysfunction in Mice Transgenic for the HD Mutation. Cell. 1997, 90 (3), 537-548.

(6) Gutekunst, C.-A.; Li, S.-H.; Yi, H.; Mulroy, J. S.; Kuemmerle, S.; Jones, R.; Rye, D.; Ferrante, R. J.; Hersch, S. M.; Li, X.-J. Nuclear and Neuropil Aggregates in Huntington's Disease: Relationship to Neuropathology. J. Neurosci. 1999, 19 (7), 2522-2534.

(7) Wagner, A. S.; Politi, A. Z.; Ast, A.; Bravo-Rodriguez, K.; Baum, K.; Buntru, A.; Strempel, N. U.; Brusendorf, L.; Hänig, C.; Boeddrich, A.; Plassmann, S.; Klockmeier, K.; Ramirez-Anguita, J. M.; Sanchez-Garcia, E.; Wolf, J.; Wanker, E. E. Self-Assembly of Mutant Huntingtin Exon-1 Fragments into Large Complex Fibrillar Structures Involves Nucleated Branching. J. Mol. Biol. 2018, 430 (12), 1725-1744.

(8) Sahoo, B.; Arduini, I.; Drombosky, K. W.; Kodali, R.; Sanders, L. H.; Greenamyre, J. T.; Wetzel, R. Folding Landscape of Mutant Huntingtin Exon1: Diffusible Multimers, Oligomers and Fibrils, and No Detectable Monomer. PLoS One. 2016, 11 (6), 1-22.

(9) Legleiter, J.; Mitchell, E.; Lotz, G. P.; Sapp, E.; Ng, C.; DiFiglia, M.; Thompson, L. M.; Muchowski, P. J. Mutant Huntingtin Fragments Form Oligomers in a Polyglutamine LengthDependent Manner in Vitro and in Vivo. J. Biol. Chem. 2010, 285 (19), 14777-14790.

(10) Landrum, E.; Wetzel, R. Biophysical Underpinnings of the Repeat Length Dependence of Polyglutamine Amyloid Formation. J. Biol. Chem. 2014, 289 (15), 10254-10260.

(11) Sahl, S. J.; Weiss, L. E.; Duim, W. C.; Frydman, J.; Moerner, W. E. Cellular Inclusion Bodies of Mutant Huntingtin Exon 1 Obscure Small Fibrillar Aggregate Species. Sci. Rep. 2012, 2 (1), 1-7.

(12) Sahl, S. J.; Lau, L.; Vonk, W. I. M.; Weiss, L. E.; Frydman, J.; Moerner, W. E. Delayed Emergence of Subdiffraction-Sized Mutant Huntingtin Fibrils Following Inclusion Body Formation. Q. Rev. Biophys. 2016, 49.

(13) Kar, K.; Jayaraman, M.; Sahoo, B.; Kodali, R.; Wetzel, R. Critical Nucleus Size for DiseaseRelated Polyglutamine Aggregation Is Repeat-Length Dependent. Nat. Struct. Mol. Biol. 2011, 18 (3), 328-336.

(14) Arrasate, M.; Mitra, S.; Schweitzer, E. S.; Segal, M. R.; Finkbeiner, S. Inclusion Body Formation Reduces Levels of Mutant Huntingtin and the Risk of Neuronal Death. Nature. 2004, 431 (7010), 805-810. 
(15) Saudou, F.; Finkbeiner, S.; Devys, D.; Greenberg, M. E. Huntingtin Acts in the Nucleus to Induce Apoptosis but Death Does Not Correlate with the Formation of Intranuclear Inclusions. Cell 1998, 95 (1), 55-56.

(16) Liu, K.-Y.; Shyu, Y.-C.; Barbaro, B. A.; Lin, Y.-T.; Chern, Y.; Thompson, L. M.; James Shen, C.K.; Marsh, J. L. Disruption of the Nuclear Membrane by Perinuclear Inclusions of Mutant Huntingtin Causes Cell-Cycle Re-Entry and Striatal Cell Death in Mouse and Cell Models of Huntington's Disease. Hum. Mol. Genet. 2015, 24 (6), 1602-1616.

(17) Bäuerlein, F. J. B.; Saha, I.; Mishra, A.; Kalemanov, M.; Martínez-Sánchez, A.; Klein, R.; Dudanova, I.; Hipp, M. S.; Hartl, F. U.; Baumeister, W.; Fernández-Busnadiego, R. In Situ Architecture and Cellular Interactions of PolyQ Inclusions. Cell. 2017, 171 (1), 179-187.e10.

(18) Olshina, M. A.; Angley, L. M.; Ramdzan, Y. M.; Tang, J.; Bailey, M. F.; Hill, A. F.; Hatters, D. M. Tracking Mutant Huntingtin Aggregation Kinetics in Cells Reveals Three Major Populations That Include an Invariant Oligomer Pool. J. Biol. Chem. 2010, 285 (28), 21807-21816.

(19) Lajoie, P.; Snapp, E. L. Formation and Toxicity of Soluble Polyglutamine Oligomers in Living Cells. PLoS One .2010, 5 (12).

(20) Lu, M.; Banetta, L.; Young, L. J.; Smith, E. J.; Bates, G. P.; Zaccone, A.; Schierle, G. S. K.; Tunnacliffe, A.; Kaminski, C. F. Live-Cell Super-Resolution Microscopy Reveals a Primary Role for Diffusion in Polyglutamine-Driven Aggresome Assembly. J. Biol. Chem. 2019, 294 (1), 257268.

(21) Nagai, Y.; Inui, T.; Popiel, H. A.; Fujikake, N.; Hasegawa, K.; Urade, Y.; Goto, Y.; Naiki, H.; Toda, T. A Toxic Monomeric Conformer of the Polyglutamine Protein. Nat. Struct. Mol. Biol. 2007, 14 (4), 332-340.

(22) hu, S.; Miller, J.; Yang, G.; Ratovitski, T.; Delannoy, M.; Muchowski, P. J.; Finkbeiner, S.; Legleiter, J.; Ross, C. A.; Poiriera, M. A. Identification of Novel Potentially Toxic Oligomers Formed in Vitro from Mammalian-Derived Expanded Huntingtin Exon-1 Protein. J. Biol. Chem. 2012, 287 (19), 16017-16028.

(23) Kim, Y. E.; Hosp, F.; Frottin, F.; Ge, H.; Mann, M.; Hayer-Hartl, M.; Hartl, F. U. Soluble Oligomers of PolyQ-Expanded Huntingtin Target a Multiplicity of Key Cellular Factors. Mol. Cell. 2016, 63 (6), 951-964.

(24) Drombosky, K. W.; Rode, S.; Kodali, R.; Jacob, T. C.; Palladino, M. J.; Wetzel, R. Mutational Analysis Implicates the Amyloid Fibril as the Toxic Entity in Huntington's Disease. Neurobiol. Dis. 2018, 120, 126-138.

(25) Pieri, L.; Madiona, K.; Bousset, L.; Melki, R. Fibrillar $\alpha$-Synuclein and Huntingtin Exon 1 Assemblies Are Toxic to the Cells. Biophys. J. 2012, 102 (12), 2894-2905.

(26) Thakur, A. K.; Jayaraman, M.; Mishra, R.; Thakur, M.; Chellgren, V. M.; Byeon, I.-J. L.; Anjum, D. H.; Kodali, R.; Creamer, T. P.; Conway, J. F.; Gronenborn, A. M.; Wetzel, R. Polyglutamine Disruption of the Huntingtin Exon $1 \mathrm{~N}$ Terminus Triggers a Complex Aggregation Mechanism. Nat. Struct. Mol. Biol. 2009, 16 (4), 380-389.

(27) Bhattacharyya, A.; Thakur, A. K.; Chellgren, V. M.; Thiagarajan, G.; Williams, A. D.; Chellgren, B. W.; Creamer, T. P.; Wetzel, R. Oligoproline Effects on Polyglutamine Conformation and Aggregation. J. Mol. Biol. 2006, 355 (3), 524-535.

(28) Darnell, G.; Orgel, J. P. R. O.; Pahl, R.; Meredith, S. C. Flanking Polyproline Sequences Inhibit $\beta$ Sheet Structure in Polyglutamine Segments by Inducing PPII-like Helix Structure. J. Mol. Biol. 2007, 374 (3), 688-704.

(29) Darnell, G. D.; Derryberry, J.; Kurutz, J. W.; Meredith, S. C. Mechanism of Cis-Inhibition of PolyQ Fibrillation by PolyP: PPII Oligomers and the Hydrophobic Effect. Biophys. J. 2009, 97 (8), 2295-2305.

(30) Mishra, R.; Jayaraman, M.; Roland, B. P.; Landrum, E.; Fullam, T.; Kodali, R.; Thakur, A. K.; Arduini, I.; Wetzel, R. Inhibiting the Nucleation of Amyloid Structure in a Huntingtin Fragment by Targeting $\alpha$-Helix-Rich Oligomeric Intermediates. J. Mol. Biol. 2012, 415 (5), 900-917. 
(31) Jayaraman, M.; Kodali, R.; Sahoo, B.; Thakur, A. K.; Mayasundari, A.; Mishra, R.; Peterson, C. B.; Wetzel, R. Slow Amyloid Nucleation via $\alpha$-Helix-Rich Oligomeric Intermediates in Short Polyglutamine-Containing Huntingtin Fragments. J. Mol. Biol. 2012, 415 (5), 881-899.

(32) Michalek, M.; Salnikov, E. S.; Werten, S.; Bechinger, B. Membrane Interactions of the Amphipathic Amino Terminus of Huntingtin. Biochemistry. 2013, 52 (5), 847-858.

(33) Mishra, R.; Hoop, C. L.; Kodali, R.; Sahoo, B.; van der Wel, P. C. A.; Wetzel, R. Serine Phosphorylation Suppresses Huntingtin Amyloid Accumulation by Altering Protein Aggregation Properties. J. Mol. Biol. 2012, 424 (1), 1-14.

(34) Kotler, S. A.; Tugarinov, V.; Schmidt, T.; Ceccon, A.; Libich, D. S.; Ghirlando, R.; Schwieters, C. D.; Clore, G. M. Probing Initial Transient Oligomerization Events Facilitating Huntingtin Fibril Nucleation at Atomic Resolution by Relaxation-Based NMR. PNAS. 2019, 116 (9), 3562-3571.

(35) Drin, G.; Antonny, B. Amphipathic Helices and Membrane Curvature. FEBS. Lett. 2010, 584 (9), 1840-1847.

(36) Burke, K. A.; Kauffman, K. J.; Umbaugh, C. S.; Frey, S. L.; Legleiter, J. The Interaction of Polyglutamine Peptides with Lipid Membranes Is Regulated by Flanking Sequences Associated with Huntingtin. J. Biol. Chem. 2013, 288 (21), 14993-15005.

Michalek, M.; Salnikov, E. S.; Bechinger, B. Structure and Topology of the Huntingtin 1-17 Membrane Anchor by a Combined Solution and Solid-State Nmr Approach. Biophys. J. 2013, 105 (3), 699-710.

Côté, S.; Binette, V.; Salnikov, E. S.; Bechinger, B.; Mousseau, N. Probing the Huntingtin 1-17 Membrane Anchor on a Phospholipid Bilayer by Using All-Atom Simulations. Biophys. J. 2015, 108 (5), 1187-1198.

Atwal, R. S.; Xia, J.; Pinchev, D.; Taylor, J.; Epand, R. M.; Truant, R. Huntingtin Has a Membrane Association Signal That Can Modulate Huntingtin Aggregation, Nuclear Entry and Toxicity. Hum. Mol. Genet. 2007, 16 (21), 2600-2615.

Chaibva, M.; Burke, K. A.; Legleiter, J. Curvature Enhances Binding and Aggregation of Huntingtin at Lipid Membranes. Biochemistry. 2014, 53 (14), 2355-2365. https://doi.org/10.1021/bi401619q.

Pandey, N. K.; Isas, J. M.; Rawat, A.; Lee, R. V.; Langen, J.; Pandey, P.; Langen, R. The 17Residue-Long $\mathrm{N}$ Terminus in Huntingtin Controls Stepwise Aggregation in Solution and on Membranes via Different Mechanisms. J. Biol. Chem. 2018, 293 (7), 2597-2605.

Chaibva, M.; Gao, X.; Jain, P.; Campbell, W. A.; Frey, S. L.; Legleiter, J. Sphingomyelin and GM1 Influence Huntingtin Binding to, Disruption of, and Aggregation on Lipid Membranes. ACS. Omega 2018, 3 (1), 273-285.

Gao, X.; Campbell, W. A.; Chaibva, M.; Jain, P.; Leslie, A. E.; Frey, S. L.; Legleiter, J. Cholesterol Modifies Huntingtin Binding to, Disruption of, and Aggregation on Lipid Membranes. Biochemistry. 2016, 55 (1), 92-102.

Beasley, M.; Stonebraker, A. R.; Hasan, I.; Kapp, K. L.; Liang, B. J.; Agarwal, G.; Groover, S.; Sedighi, F.; Legleiter, J. Lipid Membranes Influence the Ability of Small Molecules to Inhibit Huntingtin Fibrillization. Biochemistry. 2019, 58 (43), 4361-4373.

Kegel, K. B.; Sapp, E.; Alexander, J.; Valencia, A.; Reeves, P.; Li, X.; Masso, N.; Sobin, L.; Aronin, N.; DiFiglia, M. Polyglutamine Expansion in Huntingtin Alters Its Interaction with Phospholipids. J. Neurochem. 2009, 110 (5), 1585-1597.

Burke, K. A.; Hensal, K. M.; Umbaugh, C. S.; Chaibva, M.; Legleiter, J. Huntingtin Disrupts Lipid Bilayers in a PolyQ-Length Dependent Manner. Biochim. Biophys. Acta. Biomembr. 2013, 1828 (8), 1953-1961.

Harjes, P.; Wanker, E. E. The Hunt for Huntingtin Function: Interaction Partners Tell Many Different Stories. Trends. Biochem. Sci. 2003, 28 (8), 425-433.

Orr, A. L.; Li, S.; Wang, C.-E.; Li, H.; Wang, J.; Rong, J.; Xu, X.; Mastroberardino, P. G.; Greenamyre, J. T.; Li, X.-J. N-Terminal Mutant Huntingtin Associates with Mitochondria and Impairs Mitochondrial Trafficking. J. Neurosci. 2008, 28 (11), 2783-2792. 
(49) Panov, A. V.; Gutekunst, C.-A.; Leavitt, B. R.; Hayden, M. R.; Burke, J. R.; Strittmatter, W. J.; Greenamyre, J. T. Early Mitochondrial Calcium Defects in Huntington's Disease Are a Direct Effect of Polyglutamines. Nat. Neurosci. 2002, 5 (8), 731-736.

(50) Shirendeb, U.; Reddy, A. P.; Manczak, M.; Calkins, M. J.; Mao, P.; Tagle, D. A.; Hemachandra Reddy, P. Abnormal Mitochondrial Dynamics, Mitochondrial Loss and Mutant Huntingtin Oligomers in Huntington's Disease: Implications for Selective Neuronal Damage. Hum. Mol. Genet. 2011, 20 (7), 1438-1455.

(51) Ueda, M.; Li, S.; Itoh, M.; Wang, M.; Hayakawa, M.; Islam, S.; Tana; Nakagawa, K.; Chen, H.; Nakagawa, T. Expanded Polyglutamine Embedded in the Endoplasmic Reticulum Causes Membrane Distortion and Coincides with Bax Insertion.Biochem. Biophys. Res. Commun. 2016, 474 (2), 259-263.

(52) Jiang, Y.; Chadwick, S. R.; Lajoie, P. Endoplasmic Reticulum Stress: The Cause and Solution to Huntington's Disease, Brain Res. J. 2016, 1648, 650-657.

(53) Ho, C. S.; Khadka, N. K.; She, F.; Cai, J.; Pan, J. Polyglutamine Aggregates Impair Lipid Membrane Integrity and Enhance Lipid Membrane Rigidity. Biochim. Biophys. Acta. Biomembr. 2016, 1858 (4), 661-670.

(54) Qin, Z.-H.; Wang, Y.; Sapp, E.; Cuiffo, B.; Wanker, E.; Hayden, M. R.; Kegel, K. B.; Aronin, N.; DiFiglia, M. Huntingtin Bodies Sequester Vesicle-Associated Proteins by a PolyprolineDependent Interaction. J. Neurosci. 2004, 24 (1), 269-281.

(55) Kegel, K. B.; Kim, M.; Sapp, E.; McIntyre, C.; Castaño, J. G.; Aronin, N.; DiFiglia, M. Huntingtin Expression Stimulates Endosomal-Lysosomal Activity, Endosome Tubulation, and Autophagy. $J$. Neurosci. 2000, 20 (19), 7268-7278.

(56) Kegel, K. B.; Sapp, E.; Yoder, J.; Cuiffo, B.; Sobin, L.; Kim, Y. J.; Qin, Z.-H.; Hayden, M. R.; Aronin, N.; Scott, D. L.; Isenberg, G.; Goldmann, W. H.; DiFiglia, M. Huntingtin Associates with Acidic Phospholipids at the Plasma Membrane. J. Biol. Chem. 2005, 280 (43), 36464-36473.

(60) Wacker, J. L.; Zareie, M. H.; Fong, H.; Sarikaya, M.; Muchowski, P. J. Hsp70 and Hsp40 Attenuate Formation of Spherical and Annular Polyglutamine Oligomers by Partitioning Monomer. Nat. Struct. Mol. Biol. 2004, 11 (12), 1215-1222.

(61) Burke, K. A.; Godbey, J.; Legleiter, J. Assessing Mutant Huntingtin Fragment and Polyglutamine Aggregation by Atomic Force Microscopy. Methods. 2011, 53 (3), 275-284.

(62) Beasley, M.; Stonebraker, A. R.; Legleiter, J. Normalizing Polydiacetylene Colorimetric Assays of Vesicle Binding across Lipid Systems. Analytical Biochemistry. 2020, 609, 113864.

(63) Beasley, M.; Stonebraker, A. R.; Legleiter, J. Normalizing Polydiacetylene Colorimetric Assays of Vesicle Binding across Lipid Systems. Anal. Biochem. 2020, 609, 113864.

(64) Rossbach, V.; Mroszewski, K. D.; Zahn, H. Reactions of Polyamide 6 and 6,6 with 1,5-Difluoro2,4-Dinitrobenzene: Photometric Technique for the Determination of the Degree of Crosslinking. Die Makromol. Chem. 1980, 181 (10), 2071-2079.

(65) Grimm, K. M.; Joyce, J. G.; Liang, X.; Nawrocki, D. Method for Preparing a Covalently Cross Linked Oligomer of Amyloid Beta Peptides. US20100143396A1, June 10, 2010.

(66) Green, N. S.; Reisler, E.; Houk, K. N. Quantitative Evaluation of the Lengths of Homobifunctional Protein Cross-Linking Reagents Used as Molecular Rulers. Protein. Sci. 2001, 10 (7), 1293-1304. Cline, E. N.; Das, A.; Bicca, M. A.; Mohammad, S. N.; Schachner, L. F.; Kamel, J. M.; DiNunno, N.; Weng, A.; Paschall, J. D.; Bu, R. L.; Khan, F. M.; Rollins, M. G.; Ives, A. N.; Shekhawat, G.; 
Nunes-Tavares, N.; Mello, F. G. de; Compton, P. D.; Kelleher, N. L.; Klein, W. L. A Novel Crosslinking Protocol Stabilizes Amyloid $\beta$ Oligomers Capable of Inducing Alzheimer'sAssociated Pathologies. J. Neurochem. 2019, 148 (6), 822-836.

(68) Jacob, M. C.; Favre, M.; Bensa, J.-C. Membrane Cell Permeabilisation with Saponin and Multiparametric Analysis by Flow Cytometry. Cytometry. 1991, 12 (6), 550-558.

(69) Domert, J.; Sackmann, C.; Severinsson, E.; Agholme, L.; Bergström, J.; Ingelsson, M.; Hallbeck, M. Aggregated Alpha-Synuclein Transfer Efficiently between Cultured Human Neuron-Like Cells and Localize to Lysosomes. PLoS One. 2016, 11 (12).

(70) DiFiglia, M.; Sapp, E.; Chase, K.; Davies, S.; Bates, G. Aggregation of Huntingtin in Neuronal Intranculear Inclusions and Dystrophic Neurites in Brain. Science. 1997, 277 (5334), 1990-1993.

(71) Gutekunst, C. A.; Levey, A. I.; Heilman, C. J.; Whaley, W. L.; Yi, H.; Nash, N. R.; Rees, H. D.; Madden, J. J.; Hersch, S. M. Identification and Localization of Huntingtin in Brain and Human Lymphoblastoid Cell Lines with Anti-Fusion Protein Antibodies. PNAS. 1995, 92 (19), 87108714.

(72) Gutekunst, C.-A.; Li, S.-H.; Yi, H.; Ferrante, R. J.; Li, X.-J.; Hersch, S. M. The Cellular and Subcellular Localization of Huntingtin-Associated Protein 1 (HAP1): Comparison with Huntingtin in Rat and Human. J. Neurosci. 1998, 18 (19), 7674-7686.

(73) Kodali, R.; Williams, A. D.; Chemuru, S.; Wetzel, R. A $\beta(1-40)$ Forms Five Distinct Amyloid Structures Whose $\beta$-Sheet Contents and Fibril Stabilities Are Correlated. J. Mol. Biol. 2010, 401 (3), 503-517.

(74) Levy, G. R.; Shen, K.; Gavrilov, Y.; Smith, P. E. S.; Levy, Y.; Chan, R.; Frydman, J.; Frydman, L. Huntingtin's N-Terminus Rearrangements in the Presence of Membranes: A Joint Spectroscopic and Computational Perspective. ACS. Chem. Neurosci. 2019, 10 (1), 472-481.

(75) Zheng, Z.; Li, A.; Holmes, B. B.; Marasa, J. C.; Diamond, M. I. An N-Terminal Nuclear Export Signal Regulates Trafficking and Aggregation of Huntingtin (Htt) Protein Exon 1. J. Biol. Chem. 2013, 288 (9), 6063-6071.

(76) Ueda, M.; Li, S.; Itoh, M.; Hayakawa-Yano, Y.; Wang, M.; Hayakawa, M.; Hasebe-Matsubara, R.; Ohta, K.; Ohta, E.; Mizuno, A.; Hida, Y.; Matsumoto, M.; Chen, H.; Nakagawa, T. Polyglutamine Expansion Disturbs the Endoplasmic Reticulum Formation, Leading to Caspase-7 Activation through Bax.Biochem. Biophys. Res. Commun. 2014, 443 (4), 1232-1238.

(77) Choo, Y. S.; Johnson, G. V. W.; MacDonald, M.; Detloff, P. J.; Lesort, M. Mutant Huntingtin Directly Increases Susceptibility of Mitochondria to the Calcium-Induced Permeability Transition and Cytochrome c Release. Hum. Mol. Genet. 2004, 13 (14), 1407-1420.

(78) Burke, K. A.; Yates, E. A.; Legleiter, J. Amyloid-Forming Proteins Alter the Local Mechanical Properties of Lipid Membranes. Biochemistry. 2013, 52 (5), 808-817.

(79) Kim, Y. J.; Yi, Y.; Sapp, E.; Wang, Y.; Cuiffo, B.; Kegel, K. B.; Qin, Z.-H.; Aronin, N.; DiFiglia, M. Caspase 3-Cleaved N-Terminal Fragments of Wild-Type and Mutant Huntingtin Are Present in Normal and Huntington's Disease Brains, Associate with Membranes, and Undergo CalpainDependent Proteolysis. PNAS. 2001, 98 (22), 12784-12789.

(80) Leitman, J.; Ulrich Hartl, F.; Lederkremer, G. Z. Soluble Forms of PolyQ-Expanded Huntingtin Rather than Large Aggregates Cause Endoplasmic Reticulum Stress. Nat. Commun. 2013, 4 (1), 2753.

(81) Côté, S.; Wei, G.; Mousseau, N. Atomistic Mechanisms of Huntingtin N-Terminal Fragment Insertion on a Phospholipid Bilayer Revealed by Molecular Dynamics Simulations. Proteins. 2014, 82 (7), 1409-1427.

(82) Kelley, N. W.; Huang, X.; Tam, S.; Spiess, C.; Frydman, J.; Pande, V. S. The Predicted Structure of the Headpiece of the Huntingtin Protein and Its Implications on Huntingtin Aggregation. J. Mol. Biol. 2009, 388 (5), 919-927.

(83) Karanji, A. K.; Beasley, M.; Sharif, D.; Ranjbaran, A.; Legleiter, J.; Valentine, S. J. Investigating the Interactions of the First 17 Amino Acid Residues of Huntingtin with Lipid Vesicles Using Mass Spectrometry and Molecular Dynamics. J. Mass. Spectrom. 2020, 55 (1), e4470. 
Lin, H.-K.; Boatz, J. C.; Krabbendam, I. E.; Kodali, R.; Hou, Z.; Wetzel, R.; Dolga, A. M.; Poirier, M. A.; van der Wel, P. C. A. Fibril Polymorphism Affects Immobilized Non-Amyloid Flanking Domains of Huntingtin Exon1 Rather than Its Polyglutamine Core. Nat. Commun. 2017, 8 (1), 15462.

(85) Hoop, C. L.; Lin, H.-K.; Kar, K.; Magyarfalvi, G.; Lamley, J. M.; Boatz, J. C.; Mandal, A.; Lewandowski, J. R.; Wetzel, R.; Wel, P. C. A. van der. Huntingtin Exon 1 Fibrils Feature an Interdigitated $\beta$-Hairpin-Based Polyglutamine Core. PNAS. 2016, 113 (6), 1546-1551.

(86) Smith, A. N.; Märker, K.; Piretra, T.; Boatz, J. C.; Matlahov, I.; Kodali, R.; Hediger, S.; van der Wel, P. C. A.; De Paëpe, G. Structural Fingerprinting of Protein Aggregates by Dynamic Nuclear Polarization-Enhanced Solid-State NMR at Natural Isotopic Abundance. J. Am. Chem. Soc. 2018, 140 (44), 14576-14580.

(87) Isas, J. M.; Langen, R.; Siemer, A. B. Solid-State Nuclear Magnetic Resonance on the Static and Dynamic Domains of Huntingtin Exon-1 Fibrils. Biochemistry 2015, 54 (25), 3942-3949.

(88) Bugg, C. W.; Isas, J. M.; Fischer, T.; Patterson, P. H.; Langen, R. Structural Features and Domain Organization of Huntingtin Fibrils. J. Biol. Chem. 2012, 287 (38), 31739-31746.

(89) Caulkins, B. G.; Cervantes, S. A.; Isas, J. M.; Siemer, A. B. Dynamics of the Proline-Rich CTerminus of Huntingtin Exon-1 Fibrils. J. Phys. Chem. B 2018, 122 (41), 9507-9515.

(90) Sivanandam, V. N.; Jayaraman, M.; Hoop, C. L.; Kodali, R.; Wetzel, R.; van der Wel, P. C. A. The Aggregation-Enhancing Huntingtin N-Terminus Is Helical in Amyloid Fibrils. J. Am. Chem. Soc. 2011, 133 (12), 4558-4566.

(91) H, Z.; R, K.The Reactions of 1-Chloro-2,4-Dinitro-5-Fluorobenzene with the Amino Acids. Biochem Z. 1954, 325 (5), 333-338.

(92) Bányai, L.; Patthy, L. Importance of Intramolecular Interactions in the Control of the Fibrin Affinity and Activation of Human Plasminogen. J. Biol. Chem. 1984, 259 (10), 6466-6471.

(93) Ermácora, M. R.; Nowicki, C.; Wolfenstein-Todel, C.; Santomé, J. A. Identification of Intramolecular Crosslinks in Bovine Growth Hormone after Two-step Modification with 1,5difluoro-2,4-dinitrobenzene. Int. J. Pept. Protein Res. 1987, 30 (3), 423-430.

(94) Nowicki, C.; Wolfenstein-Todel, C.; Santome, J. A. Evidence for the Steric Proximity of Tyr 174 and Lys 111 in Bovine Growth Hormone. Int. J. Pept. Protein Res. 1985, 26 (6), 568-574.

(95) Marfey, P. S.; Nowak, H.; Uziel, M.; Yphantis, D. A. Reaction of Bovine Pancreatic Ribonuclease A with 1,5-Difluoro-2,4-Dinitrobenzene I. PREPARATION OF MONOMERIC INTRAMOLECULARLY BRIDGED DERIVATIVES. J. Biol. Chem. 1965, 240 (8), 3264-3269.

(96) Marfey, P. S.; Uziel, M.; Little, J. Reaction of Bovine Pancreatic Ribonuclease A with 1,5Difluoro-2,4-Dinitrobenzene II. STRUCTURE OF AN INTRAMOLECULARLY BRIDGED DERIVATIVE. J. Biol. Chem. 1965, 240 (8), 3270-3275.

(97) Cong, X.; Held, J. M.; DeGiacomo, F.; Bonner, A.; Chen, J. M.; Schilling, B.; Czerwieniec, G. A.; Gibson, B. W.; Ellerby, L. M. Mass Spectrometric Identification of Novel Lysine Acetylation Sites in Huntingtin. Mol. Cell. Proteomics. 2011, 10 (10), M111.009829-M111.009829.

(98) Arndt, J. R.; Kondalaji, S. G.; Maurer, M. M.; Parker, A.; Legleiter, J.; Valentine, S. J. Huntingtin N-Terminal Monomeric and Multimeric Structures Destabilized by Covalent Modification of Heteroatomic Residues. Biochemistry. 2015, 54 (28), 4285-4296.

(99) Matlahov, I.; van der Wel, P. C. Conformational Studies of Pathogenic Expanded Polyglutamine Protein Deposits from Huntington's Disease. Exp. Biol. Med. (Maywood). 2019, 244 (17), 15841595.

(100) Serio, T. R.; Cashikar, A. G.; Kowal, A. S.; Sawicki, G. J.; Moslehi, J. J.; Serpell, L.; Arnsdorf, M. F.; Lindquist, S. L. Nucleated Conformational Conversion and the Replication of Conformational Information by a Prion Determinant. Science. 2000, 289 (5483), 1317-1321.

(101) Hermanson, Greg T. Bioconjugate techniques. Academic press, 2013.

(102) Miller, J.; Arrasate, M.; Brooks, E.; Libeu, C. P.; Legleiter, J.; Hatters, D.; Curtis, J.; Cheung, K.; Krishnan, P.; Mitra, S.; Widjaja, K.; Shaby, B. A.; Lotz, G. P.; Newhouse, Y.; Mitchell, E. J.; Osmand, A.; Gray, M.; Thulasiramin, V.; Saudou, F.; Segal, M.; Yang, X. W.; Masliah, E.; 
Thompson, L. M.; Muchowski, P. J.; Weisgraber, K. H.; Finkbeiner, S. Identifying Polyglutamine Protein Species in Situ That Best Predict Neurodegeneration. Nat. Chem. Biol. 2011, 7 (12), 925934.

(103) Ramdzan, Y. M.; Nisbet, R. M.; Miller, J.; Finkbeiner, S.; Hill, A. F.; Hatters, D. M. Conformation Sensors That Distinguish Monomeric Proteins from Oligomers in Live Cells. Chemistry \& Biology 2010, 17 (4), 371-379.

(104) Miao, K.; Wei, L. Live-Cell Imaging and Quantification of PolyQ Aggregates by Stimulated Raman Scattering of Selective Deuterium Labeling. ACS Cent. Sci. 2020, 6 (4), 478-486.

(105) Bitan, G.; Lomakin, A.; Teplow, D. B. Amyloid $\beta$-Protein Oligomerization PRENUCLEATION INTERACTIONS REVEALED BY PHOTO-INDUCED CROSS-LINKING OF UNMODIFIED PROTEINS. J. Biol. Chem. 2001, 276 (37), 35176-35184. 


\section{Conclusion}

\subsection{Abstract}

Work presented here aimed to elucidate molecular mechanisms of huntingtin (htt) aggregation with a particular focus on the process in the presence of lipid membranes. The ultimate goal of such investigations to understand critical features of htt and its aggregate forms interacting with physiologically relevant membranes to gain insight into htt-induced toxicity in Huntington's disease (HD). Ultimately, this may lead to new targets and strategies to develop potential therapeutics to treat HD.

In Chapter 2, the impact of SUMOylation on htt aggregation in the presence and absence of lipid membranes was determined. Direct SUMOylation of Nt17 promoted an alternative aggregation pathway that leads to large, SDS soluble amorphous aggregates. These large aggregates were not membrane active. In chapter 3, the interaction of various htt aggregate species was determined, and oligomers displayed the largest membrane activity. In an attempt to further investigate the htt oligomer/bilayer interaction, a crosslinking strategy was employed that target lysine residues of Nt17. While this strategy effectively stabilized oligomers, compromising the conformational flexibility of oligomers resulted in loss of their membrane activity and cellular toxicity, suggesting that targeting the membrane activity of htt oligomers may be a viable therapeutic strategy. Importantly, such a strategy may be applicable to other neurodegenerative diseases. However, there are still several gaps in our knowledge with regard to how htt interacts with membranes that still need to be elucidated. Here, several of these gaps, and strategies to approach these issues, will be discussed. 


\subsection{The role of heterogeneous htt aggregates in toxicity}

Despite years of research in HD field, no therapies intervening in the protein-related pathology have been developed. The complexity of the htt aggregate landscape that results in a variety of distinct aggregate species complicates structure/toxic function investigations. That is, heterogeneous aggregation precludes correlation of specific aggregate states with toxic event within cells. Numerous intermediate species with relatively fast interconversion rates lead to an overlap in different aggregate states of htt. It is clear that in transgenic cellular models of HD that htt species present in cells vary widely, not only from cell to cell, but even within individual cells. Tracking the fate of specific aggregate types is exceedingly difficult. Different aggregate types may play roles in overlapping, distinct toxic events, and the process of aggregate formation may potentially serve a toxic function. The heterogeneous mixture of htt species ranges from low molecular weight species, i.e. monomers and small multimers, to intermediately sized oligomers, amorphous aggregates, and fibrils that can all ultimately coalesce into to micron-sized inclusion bodies (Fig 4.1). While there is a general consensus that polyQ length and protein concentration regulate of htt aggregation, ${ }^{1}$ various species appear to play different roles in an assortment of pathological mechanisms. Some aggregate species could represent toxic entities, while others may actually impart a protective effect. It has been challenging to study each step independently; therefore, many studies focus on global cytotoxicity that may result from a conglomeration of toxic mechanisms. Obtaining specific structure/toxic function relationships is of fundamental importance for the development of therapies targeting htt aggregation. While the formation of intranuclear or cytoplasmic inclusion bodies precedes behavioral deficits in a transgenic mouse model of $\mathrm{HD},{ }^{2}$ several observations suggest a poor correlation between inclusion body formation and HD pathology. 3,4 


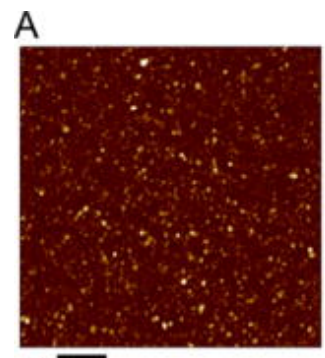

$50 \overline{0} \mathrm{~nm}$

C

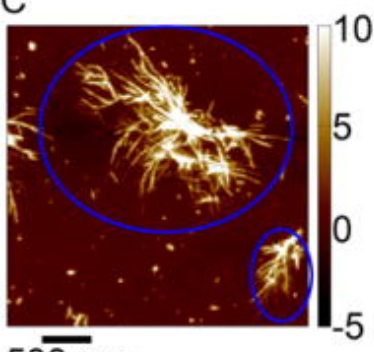

$50 \overline{0} \mathrm{~nm}$

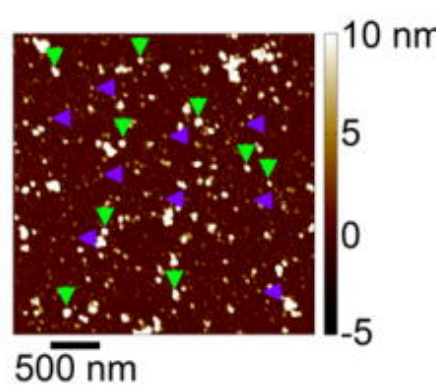

D

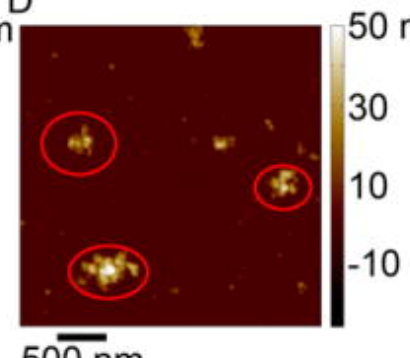

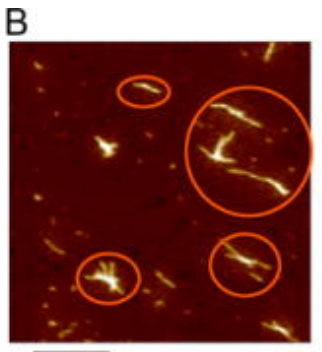

$5 \overline{00 \mathrm{~nm}}$

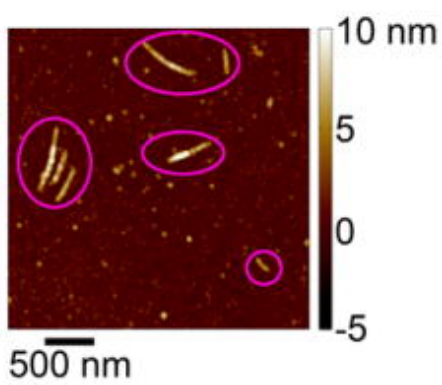

$50 \overline{\mathrm{n} m}$
E

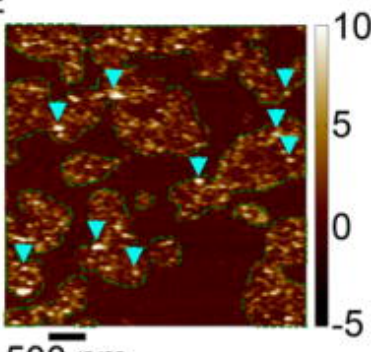

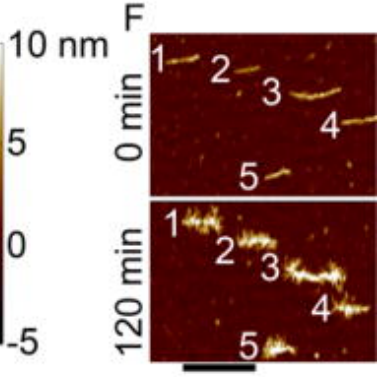

Fig 4.1 Atomic force microscopy images of a variety of aggregates formed by htt-exon1 proteins. (A) Htt exon1 can form a variety of globular, oligomeric species (purple arrows indicate oligomers $\sim 5 \mathrm{~nm}$ in height; green arrows indicate oligomers $\sim 10 \mathrm{~nm}$ in height). (B) Two morphologically distinct fibrils structures formed by exon1 (orange circles indicate thinner, smooth fibril structures; pink circles indicate thicker fibrils with a beaded morphology). (C) Blue circles indicate large bundles of htt exon1 fibrils. (D) Large, amorphous aggregates of htt exonl are indicated by red arrows (note the color scale goes up to 50 $n m$ ). (E) When htt exon1 aggregates on a lipid bilayer, a variety of oligomeric aggregates (blue arrows) associated with regions of increased membrane roughness (outlined with the green dashes lines) are observed. $(F)$ When adding monomeric htt exonl to preformed fibrils, the monomer can accumulate around the fibrils and form a variety of branching points (numbers indicated the same fibril at 0 and 120 min after exposure to monomeric htt exon1).

Inclusion body formation may function as a coping response to toxic mutant htt by sequestering the diffuse htt fraction, increasing cell survival. ${ }^{5}$ Given this potential protective role of inclusion bodies, the pathologic importance of smaller aggregate species within the diffuse htt fraction has been extensively scrutinized, as evidence points to their role in initialing toxic events. ${ }^{6}$ However, diffuse htt fraction is occupied with a variety of nanoscales aggregates species such as small tetramers to large aggregates containing 1000 - 3000 molecules, including distinct oligomers and fibrils. ${ }^{6}$ 
In various models and contexts, toxic function has been attributed to numerous, distinct htt aggregates. Initially, inclusion bodies were found to be comprised of fibril structure in post mortem brains of HD, as well as animal and cellular HD models., ${ }^{2,7}$ Mutations that promote direct formation of $\beta$-sheet rich fibrils enhance toxicity in cell culture and Drosophila models of HD, supporting the underlying amyloid structure may be key in some toxic mechanisms. ${ }^{9}$ While the interaction of insoluble fibrillar inclusion with proteins may be at the surface, the interaction of early-stage diffused aggregates of mutant htt could be multifarious with detrimental effects that may contribute to phenotype developments and neuronal dysfunction. Several different sized soluble oligomers have been postulated to be potent toxic species, ${ }^{10-12}$ and higher levels of soluble oligomers correlates with increased toxicity, independent of inclusion formation. ${ }^{11}$ Smaller soluble oligomer display abnormal interactions with over 800 cellular proteins. ${ }^{11}$ Oligomers are linked to ER stress ${ }^{12-14}$, mitochondrial fragmentation, and oxidative DNA damage. ${ }^{15}$ However, the transient nature and the heterogenicity of oligomers complicated the characterization of these species. Different oligomers may have different distinct roles in the formation and eventual elongation of fibrils. Additionally, as cellular dysfunction and toxicity likely lie in the balance between the various species, the mixture of monomeric/oligomeric/fibrillar aggregates and exogenous factors that alter aggregation are clearly of pathological significance. Identifying these cellular factors that regulate htt aggregation and evaluating the extent to which the mixture of monomeric/oligomeric/fibrillar aggregates are altered may provide valuable insight into designing HD therapies.

\subsection{Mechanistic-based approaches to investigate htt aggregation pathway and lipid membrane interaction}

While numerous factors modify htt aggregation, the flanking sequences adjacent to the polyQ domain ${ }^{16-19}$ and the presence of the lipid membranes ${ }^{20,21}$ both play a critical role in 
ultimately influencing the aggregation process. Many naturally-occuring posttranslational modifications (PTMs) within the Nt17 flanking sequence alter aggregate structure and stability. ${ }^{22-}$ ${ }^{27}$ Many of these PTMs are associated with the altered toxicity, ${ }^{23,26}$ but the majority of these studies fail to precisely characterize the impact on diffuse htt aggregate species due to limited resolution of optical microscopy employed. For example, SUMOylation reduces the formation of visible SDS-insoluble inclusions, and enhanced htt SUMOylation in Drosophila modles increases neurodegeneration, ${ }^{26}$ linking diffuse htt with toxicity. With regard to this PTM, limitations due to the diffraction limit associated with optical techniques employed did not allow for specific characterization of the nanoscale aggregates species promoted by SUMOylation, raising the question regarding what aggregates species in the diffuse fraction are promoted by SUMOylation. For this reason, in chapter 2, the resulting nanoscale aggregates formed by SUMOylated htt were characterized morphologically and biochemically. This study clarified the morphology and biophysical characteristic of nanoscale htt aggregate promoted by SUMOylaion, demonstrating that even when a fraction of the total htt has been SUMOylated that the aggregate species formed were greatly modified. In particular, SUMOylation promoted amorphous aggregates and inhibited fibril formation, suggesting that aggregates on the pathway to amorphous aggregation are benign relative to those associated with fibrillization.

Many common toxic mechanisms have been hypothesized to contribute to HD pathology (Fig 4.2). ${ }^{6,28-30}$ Many potential toxic mechanisms point to htt-exon1(46Q) disrupting of cellular and subcellular membranes, triggering a cascade of determinantal toxic effects. ${ }^{18,31}$ Additionally, concentrating htt due to subcellular localization on lipid membranes may create conditions nucleating aggregation, or the unique chemical environment associated with bilayers may promote and stabilize specific conformers involved in toxicity that would otherwise be transient. While htt 
clearly associates with lipids, ${ }^{18,32,33}$ little is known about how htt aggregation state influences these interactions.

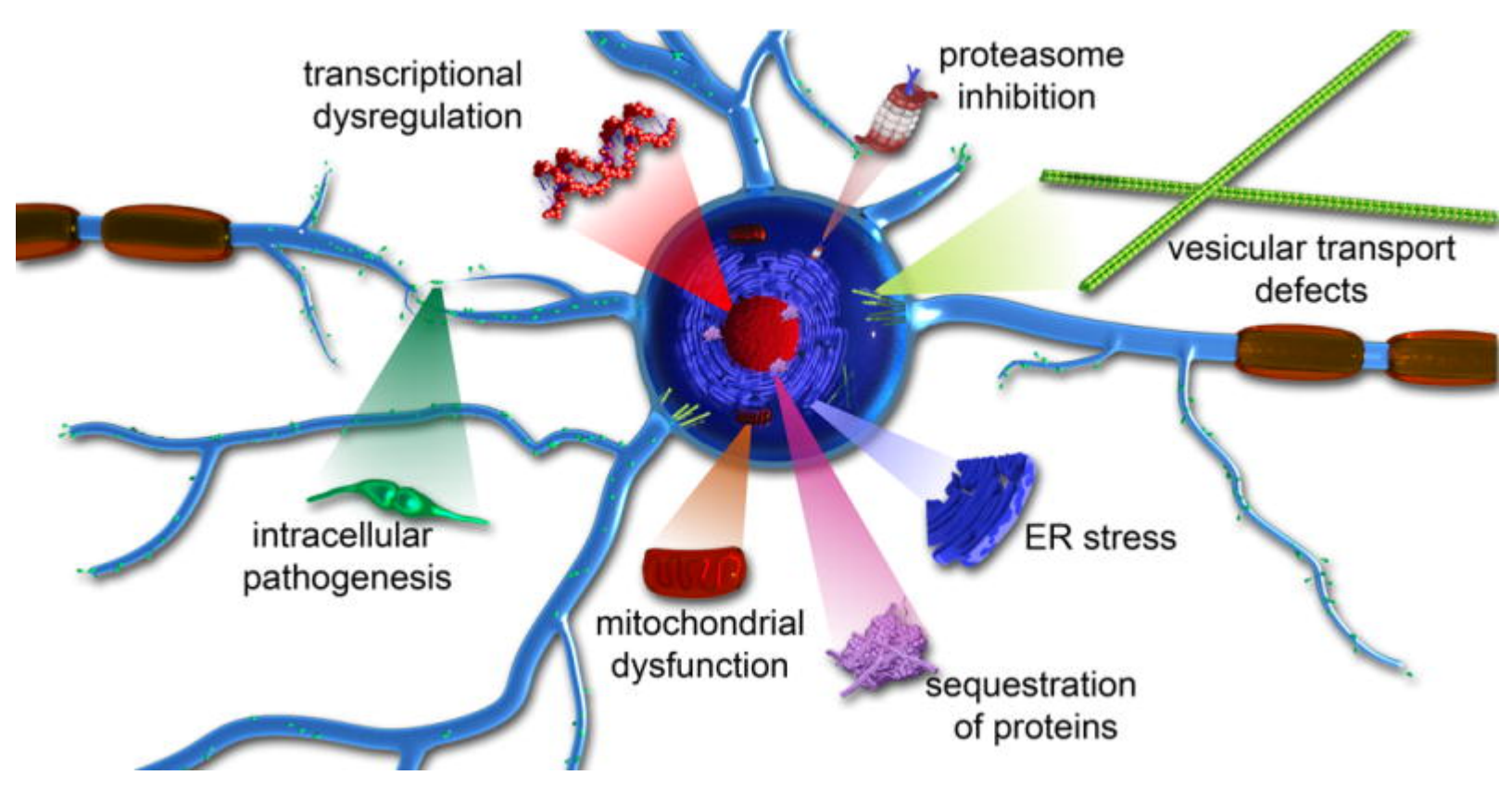

Fig 4.2 Toxic gain of function pathogenic mechanisms associated with poly $Q$ diseases.

Experiments presented in Chapter 3 demonstrated that oligomers enhance the membrane activity of htt compared to monomers, and that fibrils displayed minimal interactions with membranes. This suggests that oligomers mediate membrane-associated htt toxic mechanisms. Chemically stabilizing oligomers, however, abolishes their membrane activity and reduces htt toxicity, implying that conformational flexibility within oligomers may play a crucial role. As such, these results revealed that targeting the membrane activity of htt is a viable therapeutic strategy.

While the findings here support a role for soluble oligomers in HD pathology, crosslinking strategies have been broadly applied in attempts to elucidate the structure/toxic function properties of several amyloid-based oligomers. However, care must be taken, as Chapter 3 demonstrates that these strategies can profoundly alter the biological activity of oligomers. In the past DFDNB crosslinking technique has been used to stabilize $A \beta$ oligomers, and stabilized $A \beta$ oligomers 
increased memory dysfunction in mice. ${ }^{34}$ While the increased pathogeny of $\mathrm{A} \beta$ crosslinked oligomers is inconsistent with our observation, the disagreement may result from difference between the size distribution of crosslinked oligomers observed in each study. The stabilized htt oligomers were a more homogenous population compared to their crosslinked $A \beta$ counterparts. In $A \beta$, oligomer of different sizes are known to have various toxic potency. ${ }^{35}$ Another factor may be the innate toxicity of the DFDNB crosslinker itself.

Alternative strategies to stabilize htt oligomers may not alter their biological activity and may be considered for future structure/toxic function analysis. Other crosslinking strategies have stabilized $A \beta$ oligomers without impairing toxicity. ${ }^{34,36,37}$ For example, crosslinking $A \beta$ oligomers with glutaraldehyde resulted in a homogenous, stable oligomer population that could be purified by size-exclusion chromatography. This treatment did not cause random non-specific protein crosslinking of oligomers and, more importantly, did not alter the proteasome impairment activity of these oligomers compared to non-crosslinked $A \beta$. As protein context, i.e. flanking sequences, are known to alter htt aggregation, the addition of exogenous tags could be used to effectively stabilize htt oligomers, but care must be taken as some tags are known to alter toxicity of htt in cellular models. ${ }^{38,39}$ Targeting the oligomer-promoting Nt17 region attached to a pathogenic polyQ with another Nt17 peptide inhibits fibrillization and stabilizes oligomers. ${ }^{19,40}$ Such a strategy would not alter the conformational flexibility of the htt oligomers. Free polyP peptides added to mutant htt also stabilize htt oligomers, representing another viable option . Nevertheless, unraveling specific structure/toxic function of htt aggregates will require the ability to control the aggregation process and effectively track the fates of specific aggregate species within cells. 


\section{References}

(1) Legleiter, J.; Mitchell, E.; Lotz, G. P.; Sapp, E.; Ng, C.; DiFiglia, M.; Thompson, L. M.; Muchowski, P. J. Mutant Huntingtin Fragments Form Oligomers in a Polyglutamine LengthDependent Manner in Vitro and in Vivo. J. Biol. Chem. 2010, 285 (19), 14777-14790.

(2) Davies, S. W.; Turmaine, M.; Cozens, B. A.; DiFiglia, M.; Sharp, A. H.; Ross, C. A.; Scherzinger, E.; Wanker, E. E.; Mangiarini, L.; Bates, G. P. Formation of Neuronal Intranuclear Inclusions Underlies the Neurological Dysfunction in Mice Transgenic for the HD Mutation. Cell 1997, 90 (3), 537-548.

(3) Kuemmerle, S.; Gutekunst, C.-A.; Klein, A. M.; Li, X.-J.; Li, S.-H.; Beal, M. F.; Hersch, S. M.; Ferrante, R. J. Huntingtin Aggregates May Not Predict Neuronal Death in Huntington's Disease. Ann. Neurol. 1999, 46 (6), 842-849.

(4) Saudou, F.; Finkbeiner, S.; Devys, D.; Greenberg, M. E. Huntingtin Acts in the Nucleus to Induce Apoptosis but Death Does Not Correlate with the Formation of Intranuclear Inclusions. Cell 1998, 95 (1), 55-56.

(5) Arrasate, M.; Mitra, S.; Schweitzer, E. S.; Segal, M. R.; Finkbeiner, S. Inclusion Body Formation Reduces Levels of Mutant Huntingtin and the Risk of Neuronal Death. Nature 2004, 431 (7010), 805-810.

(6) Sahoo, B.; Arduini, I.; Drombosky, K. W.; Kodali, R.; Sanders, L. H.; Greenamyre, J. T.; Wetzel, R. Folding Landscape of Mutant Huntingtin Exon1: Diffusible Multimers, Oligomers and Fibrils, and No Detectable Monomer. Plos One 2016, 11 (6), 1-22.

(7) DiFiglia, M.; Sapp, E.; Chase, K.; Davies, S.; Bates, G. Aggregation of Huntingtin in Neuronal Intranculear Inclusions and Dystrophic Neurites in Brain. Science. 1997, 277 (5334), 1990-1993.

(8) Bäuerlein, F. J. B.; Saha, I.; Mishra, A.; Kalemanov, M.; Martínez-Sánchez, A.; Klein, R.; Dudanova, I.; Hipp, M. S.; Hartl, F. U.; Baumeister, W.; Fernández-Busnadiego, R. In Situ Architecture and Cellular Interactions of PolyQ Inclusions. Cell. 2017, 171 (1), 179-187.e10.

(9) Drombosky, K. W.; Rode, S.; Kodali, R.; Jacob, T. C.; Palladino, M. J.; Wetzel, R. Mutational Analysis Implicates the Amyloid Fibril as the Toxic Entity in Huntington's Disease. Neurobiol. Dis. 2018, 120, 126-138.

(10) Nucifora, L. G.; Burke, K. A.; Feng, X.; Arbez, N.; Zhu, S.; Miller, J.; Yang, G.; Ratovitski, T.; Delannoy, M.; Muchowski, P. J.; Finkbeiner, S.; Legleiter, J.; Ross, C. A.; Poiriera, M. A. Identification of Novel Potentially Toxic Oligomers Formed in Vitro from Mammalian-Derived Expanded Huntingtin Exon-1 Protein. J. Biol. Chem. 2012, 287 (19), 16017-16028.

(11) Kim, Y. E.; Hosp, F.; Frottin, F.; Ge, H.; Mann, M.; Hayer-Hartl, M.; Hartl, F. U. Soluble Oligomers of PolyQ-Expanded Huntingtin Target a Multiplicity of Key Cellular Factors. Mol. Cell. 2016, 63 (6), 951-964.

(12) Leitman, J.; Ulrich Hartl, F.; Lederkremer, G. Z. Soluble Forms of PolyQ-Expanded Huntingtin Rather than Large Aggregates Cause Endoplasmic Reticulum Stress. Nat. Commun. 2013, 4 (1), 2753.

(13) Jiang, Y.; Chadwick, S. R.; Lajoie, P. Endoplasmic Reticulum Stress: The Cause and Solution to Huntington's Disease, Brain. Res. J. 2016, 1648, 650-657.

(14) Ueda, M.; Li, S.; Itoh, M.; Wang, M.; Hayakawa, M.; Islam, S.; Tana; Nakagawa, K.; Chen, H.; Nakagawa, T. Expanded Polyglutamine Embedded in the Endoplasmic Reticulum Causes Membrane Distortion and Coincides with Bax Insertion.Biochem. Biophys. Res. Commun. 2016, 474 (2), 259-263.

(15) Shirendeb, U.; Reddy, A. P.; Manczak, M.; Calkins, M. J.; Mao, P.; Tagle, D. A.; Hemachandra Reddy, P. Abnormal Mitochondrial Dynamics, Mitochondrial Loss and Mutant Huntingtin Oligomers in Huntington's Disease: Implications for Selective Neuronal Damage. Hum. Mol. Genet. 2011, 20 (7), 1438-1455. 
(16) Crick, S. L.; Ruff, K. M.; Garai, K.; Frieden, C.; Pappu, R. V. Unmasking the Roles of N- and CTerminal Flanking Sequences from Exon 1 of Huntingtin as Modulators of Polyglutamine Aggregation. PNAS. 2013, 110 (50), 20075-20080.

(17) Sivanandam, V. N.; Jayaraman, M.; Hoop, C. L.; Kodali, R.; Wetzel, R.; van der Wel, P. C. A. The Aggregation-Enhancing Huntingtin N-Terminus Is Helical in Amyloid Fibrils. J. Am. Chem. Soc. 2011, 133 (12), 4558-4566.

(18) Burke, K. A.; Kauffman, K. J.; Umbaugh, C. S.; Frey, S. L.; Legleiter, J. The Interaction of Polyglutamine Peptides with Lipid Membranes Is Regulated by Flanking Sequences Associated with Huntingtin. J. Biol. Chem. 2013, 288 (21), 14993-15005.

(19) Mishra, R.; Jayaraman, M.; Roland, B. P.; Landrum, E.; Fullam, T.; Kodali, R.; Thakur, A. K.; Arduini, I.; Wetzel, R. Inhibiting the Nucleation of Amyloid Structure in a Huntingtin Fragment by Targeting $\alpha$-Helix-Rich Oligomeric Intermediates. J. Mol. Biol. 2012, 415 (5), 900-917.

(20) Gorbenko, G. P.; Kinnunen, P. K. J. The Role of Lipid-Protein Interactions in Amyloid-Type Protein Fibril Formation. Chem.Phys. Lipid. 2006, 141 (1), 72-82.

(21) Burke, K. A.; Yates, E. A.; Legleiter, J. Biophysical Insights into How Surfaces, Including Lipid Membranes, Modulate Protein Aggregation Related to Neurodegeneration. Front. Neurol. 2013, 4.

(22) Aiken, C. T.; Steffan, J. S.; Guerrero, C. M.; Khashwji, H.; Lukacsovich, T.; Simmons, D.; Purcell, J. M.; Menhaji, K.; Zhu, Y.-Z.; Green, K.; LaFerla, F.; Huang, L.; Thompson, L. M.; Marsh, J. L. Phosphorylation of Threonine 3 IMPLICATIONS FOR HUNTINGTIN AGGREGATION AND NEUROTOXICITY. J. Biol. Chem. 2009, 284 (43), 29427-29436.

(23) Thompson, L. M.; Aiken, C. T.; Kaltenbach, L. S.; Agrawal, N.; Illes, K.; Khoshnan, A.; MartinezVincente, M.; Arrasate, M.; O’Rourke, J. G.; Khashwji, H.; Lukacsovich, T.; Zhu, Y.-Z.; Lau, A. L.; Massey, A.; Hayden, M. R.; Zeitlin, S. O.; Finkbeiner, S.; Green, K. N.; LaFerla, F. M.; Bates, G.; Huang, L.; Patterson, P. H.; Lo, D. C.; Cuervo, A. M.; Marsh, J. L.; Steffan, J. S. IKK Phosphorylates Huntingtin and Targets It for Degradation by the Proteasome and Lysosome. $J$. Cell. Biol. 2009, 187 (7), 1083-1099.

(24) Kalchman, M. A.; Graham, R. K.; Xia, G.; Koide, H. B.; Hodgson, J. G.; Graham, K. C.; Goldberg, Y. P.; Gietz, R. D.; Pickart, C. M.; Hayden, M. R. Huntingtin Is Ubiquitinated and Interacts with a Specific Ubiquitin-Conjugating Enzyme. J. Biol. Chem. 1996, 271 (32), 19385-19394.

(25) Cong, X.; Held, J. M.; DeGiacomo, F.; Bonner, A.; Chen, J. M.; Schilling, B.; Czerwieniec, G. A.; Gibson, B. W.; Ellerby, L. M. Mass Spectrometric Identification of Novel Lysine Acetylation Sites in Huntingtin. Mol. Cell. Proteomics. 2011, 10 (10), M111.009829-M111.009829.

(26) Steffan, J. S.; Agrawal, N.; Pallos, J.; Rockabrand, E.; Trotman, L. C.; Slepko, N.; Illes, K.; Lukacsovich, T.; Zhu, Y.-Z.; Cattaneo, E.; Pandolfi, P. P.; Thompson, L. M.; Marsh, J. L. SUMO Modification of Huntingtin and Huntington's Disease Pathology. Science. 2004, 304 (5667), 100 104.

(27) O’Rourke, J. G.; Gareau, J. R.; Ochaba, J.; Song, W.; Raskó, T.; Reverter, D.; Lee, J.; Monteys, A. M.; Pallos, J.; Mee, L.; Vashishtha, M.; Apostol, B. L.; Nicholson, T. P.; Illes, K.; Zhu, Y.-Z.; Dasso, M.; Bates, G. P.; Difiglia, M.; Davidson, B.; Wanker, E. E.; Marsh, J. L.; Lima, C. D.; Steffan, J. S.; Thompson, L. M. SUMO-2 and PIAS1 Modulate Insoluble Mutant Huntingtin Protein Accumulation. Cell .Rep. 2013, 4 (2), 362-375.

(28) Benn, C. L.; Sun, T.; Sadri-Vakili, G.; McFarland, K. N.; DiRocco, D. P.; Yohrling, G. J.; Clark, T. W.; Bouzou, B.; Cha, J.-H. J. Huntingtin Modulates Transcription, Occupies Gene Promoters In Vivo, and Binds Directly to DNA in a Polyglutamine-Dependent Manner. J. Neurosci. 2008, 28 (42), 10720-10733.

(29) Bolognesi, B.; Kumita, J. R.; Barros, T. P.; Esbjorner, E. K.; Luheshi, L. M.; Crowther, D. C.; Wilson, M. R.; Dobson, C. M.; Favrin, G.; Yerbury, J. J. ANS Binding Reveals Common Features of Cytotoxic Amyloid Species. ACS Chem. Biol. 2010, 5 (8), 735-740.

(30) Balch, W. E.; Morimoto, R. I.; Dillin, A.; Kelly, J. W. Adapting Proteostasis for Disease Intervention. Science. 2008, 319 (5865), 916-919. 
(31) Gao, X.; Campbell, W. A.; Chaibva, M.; Jain, P.; Leslie, A. E.; Frey, S. L.; Legleiter, J. Cholesterol Modifies Huntingtin Binding to, Disruption of, and Aggregation on Lipid Membranes. Biochemistry. 2016, 55 (1), 92-102.

(32) Burke, K. A.; Hensal, K. M.; Umbaugh, C. S.; Chaibva, M.; Legleiter, J. Huntingtin Disrupts Lipid Bilayers in a PolyQ-Length Dependent Manner. Biochim. Biophys. Acta. Biomembr. 2013, 1828 (8), 1953-1961.

(33) Côté, S.; Binette, V.; Salnikov, E. S.; Bechinger, B.; Mousseau, N. Probing the Huntingtin 1-17 Membrane Anchor on a Phospholipid Bilayer by Using All-Atom Simulations. Biophys. J. 2015, 108 (5), 1187-1198.

(34) Cline, E. N.; Das, A.; Bicca, M. A.; Mohammad, S. N.; Schachner, L. F.; Kamel, J. M.; DiNunno, N.; Weng, A.; Paschall, J. D.; Bu, R. L.; Khan, F. M.; Rollins, M. G.; Ives, A. N.; Shekhawat, G.; Nunes-Tavares, N.; Mello, F. G. de; Compton, P. D.; Kelleher, N. L.; Klein, W. L. A Novel Crosslinking Protocol Stabilizes Amyloid $\beta$ Oligomers Capable of Inducing Alzheimer'sAssociated Pathologies. J. Neurochem. 2019, 148 (6), 822-836.

(35) Iv, A. W. P.; Legleiter, J. Challenges in Understanding the Structure/Activity Relationship of A $\beta$ Oligomers. AIMS. Biophys. 2019, 6 (1), 1.

(36) Thibaudeau, T. A.; Anderson, R. T.; Smith, D. M. A Common Mechanism of Proteasome Impairment by Neurodegenerative Disease-Associated Oligomers. Nat. Commun. 2018, 9 (1), 1097.

(37) Ono, K.; Condron, M. M.; Teplow, D. B. Structure-Neurotoxicity Relationships of Amyloid $\beta$ Protein Oligomers. PNAS. 2009, 106 (35), 14745-14750.

(38) Jiang, Y.; Gregorio, S. E. D.; Duennwald, M. L.; Lajoie, P. Polyglutamine Toxicity in Yeast Uncovers Phenotypic Variations between Different Fluorescent Protein Fusions. Traffic. 2017, 18 (1), 58-70.

(39) Duennwald, M. L.; Jagadish, S.; Muchowski, P. J.; Lindquist, S. Flanking Sequences Profoundly Alter Polyglutamine Toxicity in Yeast. Pro.c Nat. Acad. Sci. USA. 2006, 103 (29), 11045-11050.

(40) Arndt, J. R.; Chaibva, M.; Beasley, M.; Kiani Karanji, A.; Ghassabi Kondalaji, S.; Khakinejad, M.; Sarver, O.; Legleiter, J.; Valentine, S. J. Nucleation Inhibition of Huntingtin Protein (Htt) by Polyproline PPII Helices: A Potential Interaction with the N-Terminal $\alpha$-Helical Region of Htt. Biochemistry. 2020, 59 (4), 436-449. 